


\section{SCALABLE BROADCAST AUTHENTICATION FOR V2V COMMUNICATION}

MICHAEL FEIRI 


\section{GRADUATION COMMITTEE}

Prof. dr. P.M.G. Apers University of Twente, The Netherlands

Prof. dr. F.E. Kargl University of Ulm, Germany, and University of Twente, The Netherlands

Dr. J.Y. Petit Security Innovation Inc., U.S.A.

Prof. dr. P.H. Hartel University of Twente, The Netherlands

Prof. dr. A. Pras University of Twente, The Netherlands

Prof. dr. S. Etalle Technical University of Eindhoven and University of Twente, The Netherlands

Prof. dr. F. Dressler Paderborn University, Germany

Dr.-ing. R.K. Schmidt Denso Automotive GmbH, Germany

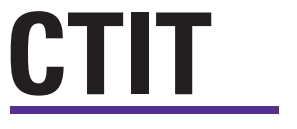

CTIT Ph.D. Thesis Series No. 16-407

Centre for Telematics and

Information Technology

P.O. Box 217, 7500 AE

Enschede, The Netherlands

ISBN: 978-90-365-4200-5

ISSN: $1381-3617$ (CTIT Ph.D. Thesis Series No. 16-407)

DOI: 10.3990/1.9789036542005

Typeset with LTEX.

Printed by Gildeprint Drukkerijen

Cover design by dr. Stefan Dietzel

Copyright (C) 2016, Michael Feiri

All rights reserved. No part of this book may be reproduced or transmitted in any form or by any means, electronic or mechanical, including photography, recording, or any information storage and retrieval system, without prior written permission of the author. 


\title{
SCALABLE BROADCAST \\ AUTHENTICATION FOR V2V \\ COMMUNICATION
}

\author{
PROEFSCHRIFT
}

ter verkrijging van

de graad van doctor aan de Universiteit Twente,

op gezag van de rector magnificus,

Prof.dr. H. Brinksma,

volgens besluit van het College voor Promoties

in het openbaar te verdedigen

op 13 October 2016 om 16:45

door

MICHAEL PETER FEIRI

geboren op 25 April 1979

te Friedrichshafen, Duitsland 
Dit proefschrift is goedgekeurd door:

Prof. dr. F.E. Kargl (Promotor)

Dr. J.Y. Petit (Co-Promotor) 
Vehicular Ad Hoc Networking (VANET) technology is, at it's core, the simple idea of outfitting vehicles with wireless data communication equipment for automatic information exchange. This technology is expected to serve as a foundation for a set of novel safety, automation, and infotainment applications. The most prominent among these applications are expected to be driver assistance systems which also support advanced levels of automated driving. These applications stand to benefit from enhanced situational awareness, which is made possible through the cooperative exchange of information about environmental influences and the presence and condition of surrounding vehicles.

Wireless networking technology and networking in general are well understood domains in computer science. However, the context of connected vehicles and the associated requirements and communication patterns imposes a set of unique challenges, which require solutions that differ from established networking practices. The susceptibility of wireless communication to packet loss and the very high mobility of vehicular communication nodes make VANET technology extremely volatile. At the same time the usage in safety critical applications demands very low latency and high availability of the communication infrastructure for frequent information exchange. And on top of these challenges security and privacy need taken into account in the design of the overall communication infrastructure. Classic solutions for stable networks cannot provide optimal performance characteristics under these conditions.

The focus of this work is specifically on vehicle-to-vehicle technology $\left(\mathrm{V}_{2} \mathrm{~V}\right)$, which is a subset of the more general vehicle-to-anything $\left(\mathrm{V}_{2} \mathrm{X}\right)$ topic. This subset of VANET is concerned with the direct information exchange among vehicles without the involvement of additional infrastructure, which may or may not be available to vehicles which driving. Direct $\mathrm{V}_{2} \mathrm{~V}$ communication is expected to always be available between vehicle within a safety critical range. Therefore, this communication path is expected to be used to enable the most safety critical applications.

The scalability of security solutions for vehicular communication remains an untested aspect of ongoing efforts to bring VANET technology to the market on a larger scale. Filed operational test projects have started to trial VANET deployments to investigate, but penetration rates are too low to allow for realistic extrapolations of future scalability problems. This dissertations contributes to the research efforts that support the development of secure vehicular communication tech- 
nology through investigations of attributes and solutions for scalable security for $\mathrm{V} 2 \mathrm{~V}$ broadcast communication.

Part II reviews security requirements and provides detailed quantifications of performance requirements for security in $\mathrm{V}_{2} \mathrm{~V}$ broadcast communication. These requirements define the solution space for applicable broadcast authentication techniques. Additionally, the review of achievable security and privacy goals enables informed trade-offs between security and privacy in the context of effective and efficient pseudonymity schemes. Finally, an information flow analysis shows the broader need to consider attacker models beyond the classic network oriented view, in order to capture the full spectrum of the threat landscape for connected vehicle technology.

Part III contributes a study of hardware assisted scalability solutions for the relevant cryptographic algorithms in $\mathrm{V}_{2} \mathrm{~V}$ broadcast authentication. This specifically concerns the performance characteristics of dedicated hardware security modules and the feasibility of reaching sufficient performance levels to satisfy the requirements of the expected communication patterns in vehicular environments. A second contribution under the topic of hardware assisted scalability solutions is a novel storage systems for pseudonymous identities. An application of Physically Inclinable Functions (PUF) allows for very efficient and secure storage of large sets of private key material, as it is expected to be used for privacy protection on vehicular communication.

Part IV contributes detailed simulation studies of the costs and benefits of in-line certificate management in the $\mathrm{V}_{2} \mathrm{~V}$ communication channel with a focus on scalability. The increased communication load due to the inclusion of certificate material can cause availability problems in highly congested situations. Proposals for certificate omission schemes exist, but do not sufficiently take scalability in extremely congested situations into account. A novel congestion-based certificate omission scheme is proposed and evaluated in simulation studies. Additionally, a novel certificate pre-distribution approach is proposed, which is permissible under the assumptions of achievable privacy and can offer enhanced availability during privacy preserving pseudonym changes. 
PUBLICATIONS

Some content has appeared previously in the following publications:

- Feiri, M. and Petit, J. and Kargl, F. (2012) Congestion-based Certificate Omission in VANETs. ACM International Workshop on Vehicular Ad Hoc Networks (VANET 2012), 25 June 2012, Low Wood Bay, Lake District, United Kingdom

- Petit, J. and Bösch, C. and Feiri, M. and Kargl, F. (2012) On the Potential of PUF for Pseudonym Generation in Vehicular Networks. IEEE Vehicular Networking Conference (VNC 2012), 14-16 Nov 2012, Seoul, Korea

- Feiri, M. and Petit, J. and Kargl, F. (2012) Evaluation of Congestionbased Certificate Omission in VANETs. IEEE Vehicular Networking Conference (VNC 2012), 14-16 Nov 2012, Seoul, Korea

- Feiri, M. and Petit, J. and Kargl, F. (2013) Efficient and secure storage of private keys for pseudonymous vehicular communication. ACM Workshop on Security, Privacy and Dependability for CyberVehicles (CyCar 2013), 04 Nov 2013, Berlin, Germany

- Feiri, M. and Petit, J. and Schmidt, R. and Kargl, F. (2013) The impact of security on cooperative awareness in VANET. IEEE Vehicular Networking Conference (VNC 2013), 16-18 Dec 2013, Boston, USA

- Feiri, M. and Petit, J. and Kargl, F. (2014) Real Wold Privacy Expectations in VANETs. GI/ITG KuVS Fachgespräch Inter-Vehicle Communication (FG-IVC 2014), 20-21 Feb 2014, Luxembourg City, Luxembourg

- Feiri, M. and Petit, J. and Kargl, F. (2014) An evaluation framework for pre-distribution strategies of certificates in VANETs. GI/ITG KuVS Fachgespräch Inter-Vehicle Communication (FGIVC 2014), 20-21 Feb 2014, Luxembourg City, Luxembourg

- Petit, J. and Feiri, M. and Kargl, F. (2014) Revisiting Attacker Model for Smart Vehicles. IEEE International Symposium on Wireless Vehicular Communications (WiVec 2014), 14-15 Sep 2014, Vancouver, Canada

- Feiri, M. and Petit, J. and Kargl, F. (2014) Formal Model of Certificate Omission Schemes in VANET. IEEE Vehicular Networking Conference (VNC 2014) , 3-5 Dec 2014, Paderborn, Germany 
- Petit, J. and Schaub, F. and Feiri, M. and Kargl, F. (2015) Pseudonym Schemes in Vehicular Networks: A Survey. IEEE Communications Surveys \& Tutorials, Volume 17

- Feiri, M. and Petit, J. and Kargl, F. (2015) The case for announcing pseudonym changes. GI/ITG KuVS Fachgespräch Inter-Vehicle Communication (FG-IVC 2015), 19-20 Mar 2015, Ulm, Germany

- Feiri, M. and Pielage, R. and Petit, J. and Zannone, N. and Kargl, F. (2015) Pre-distribution of Certificates for Pseudonymous Broadcast Authentication in VANET. IEEE Vehicular Technology Conference (VTC2015-Spring), 11-14 May 2015, Glasgow, Scotland

- Petit, J. and Broekhuis, D. and Feiri, M. and Kargl, F. (2015) Connected Vehicles: I Can Track You! BlackHat Europe 2015, 10-13 November 2015, Amsterdam, The Netherlands

- Petit, J. and Stottelaar, B. and Feiri, M. and Kargl, F. (2015) Selfdriving cars: Don't trust your sensors. BlackHat Europe 2015, 10-13 November 2015, Amsterdam, The Netherlands 


\section{CONTENTS}

I BROADCAST AUTHENTICATION IN VANET I

I INTRODUCTION 3

1.1 Motivation 3

1.2 Scope 5

1.3 Research objectives 6

II REASSESSMENT OF REQUIREMENTS 9

2 OVERVIEW II

3 GOALS AND CONSTRAINTS 15

3.1 Security Requirements 15

3.2 Achievable Privacy 16

3.2.1 Local observer 19

3.2.2 Global observer 20

3.2.3 Medium observer 20

3.3 Quantifying Bandwidth Constraints 21

3.4 Quantifying Performance Constraints 24

4 ATTACKER MODELS 29

4.1 Data Lifecycle 29

4.2 Revisited Attacker Model 31

4.2.1 Sensor Confusion 31

4.2.2 Evil Mechanic 33

4.2.3 Communication Attacks 36

5 SUMMARY AND CONCLUSION 39

III HARDWARE SOLUTIONS 41

6 OVERVIEW 43

7 PSEUDONYM STORAGE IN PHYSICALLY UNCLONABLE FUNC-

TIONS 47

7.1 Related work 48

7.1.1 PKI-based pseudonym provisioning 48

7.1.2 Autonomous pseudonym provisioning 49

7.1.3 Scalable secure key storage 50

7.2 System Model 50

7.2.1 Physical Unclonable Functions 50

7.2.2 On-Board Unit Architecture 51

7.2.3 Attacker Model 53

$7 \cdot 3 \quad$ Classic secure storage 54

7.3.1 Individual key storage 54

7.3.2 Encrypted storage 55 
7.3.3 Key derivation 56

$7 \cdot 4 \quad$ PUF-based secure storage $\quad 57$

7.4.1 Strong PUF-based secure storage 57

7.4.2 Weak PUF-based key derivation 60

$7 \cdot 5$ Discussion 63

7.5.1 Limitations of KDFs and PUFs 65

7.5.2 PUF integrated within an HSM 66

7.6 Conclusion 67

8 ACCELERATED CRYPTOGRAPHY FOR SECURE VEHICULAR COMMUNICATION 69

8.1 A representative vehicular security subsystem 69

8.2 Platforms for on-board units 73

8.3 Latency and throughput 73

8.4 Peak software performance 76

8.5 Cryptographic hardware acceleration 80

8.6 Conclusions 81

9 CONCLUSION 83

IV CERTificate MANAgEMENT 85

IO OVERVIEW 87

11 CERTIFICATE OMISSION 91

11.1 Channel and system model 93

11.2 Related work 96

11.3 Simulation model and analytical model IOI

12 CONGESTION BASED CERTIFICATE OMISSION 115

12.1 Congestion-based Certificate Omission Scheme 115

12.2 Evaluation 118

12.2.1 Analysis 120

12.2.2 Comparison with other Omission schemes 125

12.2.3 Discussion 128

13 Application LeVel eVAluation 135

13.1 Awareness Quality 136

13.2 Awareness Quality under Certificate Omission ${ }_{138}$

13.2.1 AQL Measurements 139

14 CERTIFICATE PRE-DistRIBUTION 151

14.1 An initial assessment 151

14.2 Summary and future work 160

15 SUMMARY AND FUTURE WORK 163

V CONCLUSION AND FUTURE WORK 167

16 SUMMARY OF CONTRIBUTIONS 169

17 FUTURE RESEARCH DIRECTIONS 175

BIBLIOGRAPHY 181 
Figure 3.1 Time requirements for CAM generation and CAM processing according to ETSI ITS [39] 24

Figure 3.2 Maximum message throughput over a single 802.11p channel as measured in simulations by the PRESERVE project [133] 26

Figure $4 \cdot 1$ Abstract model of data lifecycle stages in smart vehicle domain 30

Figure 6.1 A prototype HSM device for use in secure vehicular communication, made by the european

Figure 7.1 research project PRESERVE 46 ETSI architecture of an OBU [32] 52

Figure 7.2 Simplified hardware architecture of an OBU

Figure 7.3

Figure 7.4 All keys in secure storage 55

Keys retrieved from encrypted file in regular storage using a securely stored master key $\quad 56$

Figure 7.5 Keys regenerated through a key derivation function using a securely stored master key $\quad 56$

Figure 7.6 Keys reconstructed securely from a strong PUF using regularly stored challenges and helper data 58

Figure 7.7 An initial challenge (C) gets expanded into $n$ challenges $\left(c_{i}\right)$, which generate responses $\left(r_{i}\right)$ in the PUF. The vehicle combines these into a final response (R) and helper data (W). $\quad 59$ Figure 7.8 Regeneration of responses is analogous to the initial provisioning, except the previously generated helper data $(W)$ is now utilized by the Stabilise() function to stabilize the response.

Figure 7.9 The vehicle generates an asymmetric key pair from a challenge $C$ and helper data $W$. The $C A$ creates a certificate for the public key $p k$, which is stored in the vehicle with $C$ and $W$. $\quad 60$

Figure 7.10 A master key gets reconstructed securely from a weak PUF using regularly stored challenges and helper data and is then used to regenerate derived keys. 61

Figure 8.1 Architecture of the PRESERVE V2X Security Subsystem [13] 70

Figure 8.2 Control flow of incoming message verification in the PRESERVE VSSKit [13] 71 
Figure 8.3

Figure 8.4

Figure 11.1

Figure 11.2

Figure 11.3

Figure 11.4

Figure 11.5

Figure 11.6

Figure 11.7

Figure 11.8

Figure 11.9

Figure 11.10

Figure 11.11

Figure 11.12

Figure 11.13

Figure 11.14

Figure 12.1

Figure 12.2
The tested on-board units in various laboratory settings $\quad 74$

Signature verification times for 5000 messages, showing the extent of jitter on a NEXCOM VTC 6201 running 32bit Ubuntu 10.04 Linux

Repetition of secure messages in vehicular communication over time. Only the data and signature will change between messages. $\quad 92$ Secure message transmission with deliver of some certificates omitted 93

Packet delivery rates in scenarios without load in the communication channel follow ideal algorithmic probability patterns 108

Packet delivery rates in scenarios with high load in the communication channel suffer from diverse rates of packet loss 108

Polynomially fitted packet delivery rate without load in the communication channel 109 Polynomially fitted packet delivery rate with high load in the communication channel 109 Certificate delivery rate mc without load in the communication channel 110

Certificate delivery rate mc with high load in the communication channel 110

Probability of certificate reception after $n$ beacon periods without load in the communication channel at 30om distance 111

Probability of certificate reception after $n$ beacon periods with high load in the communication channel at 30om distance 111

Overall packet delivery rate considering CPL and NPL without load in the communication channel at 30om distance 112

Overall packet delivery rate considering CPL and NPL with high load in the communication channel at 30om distance 112

Overall packet delivery rate considering CPL and NPL with high load in the communication channel at 200m distance 113

Overall packet delivery rate considering CPL and NPL with high load in the communication channel at 1oom distance 113

Example of $\mathrm{CbCO} \quad 117$

Connectivity measurements in the selected scenario 121 
Figure 12.3

Figure 12.4

Figure 12.5

Figure 12.6

Figure 12.7

Figure 12.8

Figure 12.9

Figure 12.10

Figure 12.11

Figure 12.12

Figure 12.13

Figure 12.14

Figure 12.15

Figure 12.16

Figure 13.1

Figure 13.2

Figure 13.3

Figure 13.4
Omission rates strategies for congestion-based certificate omission 122

Average percentage of certificate omissions in CbCO 123

Average percent of unverifiable messages among received messages 124

Increase of packet loss due to inclusion of certificates for different variants of $\mathrm{CbCO}$ (NPL only) $\quad 125$

Increase of packet loss due to inclusion of certificates for different variants of $\mathrm{CbCO}$, counting NPL + CPL $\quad 126$

Illustration of the effect of combining cryptographic packet loss with regular network packet loss $\quad 127$

Maximum number of unverifiable beacons until arrival of certificate $\quad 128$

Average number of unverifiable beacons until arrival of certificate 129

Average number of unverifiable beacons until arrival of certificate 130

Average percentage of certificate omission in other protocols 131

Average percent of unverifiable packets for various proposed omission schemes 131

Increase of packet loss due to inclusion of certificates for different omission schemes (NPL only) $\quad 132$

Increase of packet loss due to inclusion of certificates for different omission schemes, counting NPL + CPL $\quad 132$

Increase of packet loss due to inclusion of certificates for different omission schemes, counting NPL + CPL. NoOm serves as a reference for no omissions 133

Average AQL in areas of $100 \mathrm{~m}$ width around vehicles in the low density scenario 140 Average AQL in areas of $100 \mathrm{~m}$ width around vehicles in the high density scenario 141 Average AQL for a safety area of $0 \mathrm{~m}$ to $100 \mathrm{~m}$ around vehicles under varying numbers of vehicles 142

Average AQL for a safety area of $0 \mathrm{~m}$ to $300 \mathrm{~m}$ around vehicles under varying numbers of vehicles 143 
Figure 13.5

Figure 13.6

Figure 13.7

Figure 13.8

Figure 13.9

Figure 14.1

Figure 14.2

Figure 14.3

Figure 14.4

Figure 14.5
AQL measurement during the first 200 beacon periods of a high load simulation at a sampling rate of 1 per beacon cycle 144

AQL measurement during the first 200 beacon periods of a high load simulation at a sampling rate of 1 per beacon cycle, not considering unverifiable packets as lost packets $\quad 145$ AQL measurement during the first 30 beacon periods of a high load simulation at a sampling rate of 1 per beacon cycle $\quad 146$

Comparative AQL measurement of $\mathrm{CbCO}$ linear and $\mathrm{CbCO}$ quad during the first 30 beacon periods of a high load simulation at a sampling rate of 1 per beacon cycle 148

AQL measurement during the first 30 beacon periods of a low load simulation at a sampling rate of 1 per beacon cycle 149

Congestion-based Certificate Omission reduces inclusion of certificates when congestion in the communication channel increases 153

Geographic $(\mathrm{G})$ and Temporal $(\mathrm{T})$ certificate pre-distribution through certificate concatenation on top of Congestion-based Certificate Omission 154

Awareness quality without and with geographic pre-distribution 157

Awareness quality without and with temporal pre-distribution 159

Awareness quality without and with mix predistribution 160 
Table 4.1

Table 7.1

Table 7.2

Table 8.1

Table 8.2

Table 8.3

Table 8.4

Table 11.1

Table 11.2

Table 11.3

Table 12.1

Table 12.2

Table 13.1

Table 13.2

Table 14.1
Attackers in the data lifecycle 30

Storage size overview for k keys 62

Key stealing protection under different attacker capabilities $\quad 64$

Platform and compiler properties $\quad 72$

Single core sign and verify test results with PRESERVE VSSKit vi.4 using the OpenSSL backend 73

Peak ECDSA NISTP256 latency and throughput values for OpenSSL 1.o.2a on the Nexcom VTC 6201 platform. Test were performed within a single threaded VSSKit 2 and with a synthetic multi threaded benchmark tool that skips all message processing beyond raw signature creation and verification. $\quad 77$

An overview of several hardware acceleration solutions for ECDSA signature verification 79

Message specifications 95

System model assumptions $\quad 96$

Simulation parameters $\quad 102$

Cryptographic settings 119

Simulation parameters 120

Omission Schemes 139

Performance of Omission Schemes $\quad 150$

Pre-distribution settings $\quad 156$ 

Part I

BROADCAST AUTHENTICATION IN VANET 

INTRODUCTION

\subsection{MOTIVATION}

As a means of individual transportation the use of private vehicles provides autonomy and freedom of movement for their users. The network of public roads is however a shared environment. Cooperation between drivers is required to ensure smooth traffic flow and to avoid accidents. The basic foundations of this collaboration are visual perception by the drivers, contextual assumptions about the intent of other drivers, knowledge of regulatory constraints upon the permitted usage types in designated areas, as well as explicit visual- and auditory-signaling such as turn-signal lights or "honking" as a warning signal.

Traditionally the development of vehicle technology has centered around the efficiency of converting stored energy into motion. Advances in aerodynamics, materials and engine technology have enhanced the range, comfort, and affordability of vehicles for individual transportation. These technological advances have allowed automobiles to emerge as the major transportation solution for large parts of the public. However, the success of the automobile as a solution for large-scale individual transportation makes it increasingly more difficult to achieve the aforementioned basic goals of transportation on shared public roads: Smooth traffic flow (efficiency) and avoidance of accidents (safety).

The increasing density of vehicles on public roads requires increased efforts to maintain (and indeed improve) both safety and traffic-flow. The importance of safety is self-evident as the loss of life and injuries have a clear negative impact on society at large - in addition, the accompanying economic damage is quantifiable. Ensuring smooth traffic flow appears to be a slightly more qualitative goal, which nevertheless goes beyond simply driver comfort. Traffic congestion has direct economic impacts and also increases the ecological footprint of automobile use through wasteful consumption of energy.

A number of vehicle safety features have already been developed to maintain and improve safety of automobile traffic despite an increase of traffic density. A broad classification distinguishes between "active- 
safety" features and "passive-safety" features. Active safety features take over part of the control of the vehicle from the driver, to either avoid accidents completely or at least minimize the effects of an accident. Such features include braking assistants, anti-lock braking, stability control, traction control and cruise control. Passive safety features offer increased protection for vehicle passengers (and more recently pedestrians) during and after a crash. Examples for such features include deformation zones, seat belts, air bags, laminated glass, hoodlifting devices and the positioning of critical components such as fuel tanks within the vehicle. Due to the distinction based on the temporal position relative to the occurrence of a crash, these features are sometimes also be referred to as "pre-crash" and "post-crash" safety features, although this indicates a certain inevitability to the crash situation!

All safety systems benefit from situational-awareness, derived from sensor data. This is true for active driver assistance features, but also holds true for some passive safety features. Impact sensors can, for example, trigger components of passive safety features such as the tensioning of seat belts or the release of airbags. The appearance of actual situational-awareness of a vehicle's electronic safety systems even led to the label "intelligent vehicles", although even complex situationalawareness is unlikely to lead directly to intelligence which includes self-awareness. However, it is unquestionable that the availability of sensor data about a vehicle's surroundings does enable powerful safety features.

The capacity and precision of local sensors is, however, limited by their operational range and secondary factors such as shadowing or weather conditions. The exchange of sensor information over wireless data communication channels can provide a significant enhancement over purely local sensor data collection. The exchange of sensor data measurements with surrounding vehicles or the usage of sensor data provided by road infrastructure can greatly enhance the range and accuracy of a vehicles situational awareness. A "telematic horizon", which can far exceed the range of local sensors, opens up possibilities for better calibration of passive security features, more accurate collision avoidance, and possibly novel safety features.

Exchanging and accepting remote sensor data into a vehicle's local model of the surrounding environment marks the beginning of complex cooperation between computer-aided vehicles. Cooperative maneuvers involving multiple vehicles can be scheduled and executed based on the data that is exchanged among nominally independent vehicles. This type of cooperation makes this a prime example of machine-to-machine communication. The fact that the shared information is directly consumable by machines is a qualitative leap that also supports scenarios that eliminate human drivers. Autonomous driving 
is commonly classified as a final stage of driver assistance features. Considering fully autonomous driving as a final extension stage of an active safety feature highlights the fact that the elimination of the human factor in transportation can be seen as a safety feature. The expectation being that cybernetic control systems act in a more rational and predictable fashion than human drivers. From this perspective it is also clear that any feature that enhances driver assistance, such as intervehicular communication, is naturally useful to enhance autonomous driving as well.

Looking beyond the utility of vehicular communication for the individual driver it also becomes apparent that the possibility of larger-scale coordination can be another motivation for cooperative information exchange among vehicles. Examples for such coordination range from speed optimization for approaches to traffic lights, to swarming behavior and platooning patterns for minimization of fuel consumption, up to macroscopic traffic flow optimizations. For the most abstract highlevel coordination it might become necessary to extend communication patterns to cover multi-hop dissemination or to utilize support from backend infrastructure services. A macroscopic intelligent transportation system (ITS) can exploit detailed knowledge of vehicle presence to optimize traffic flow through adjusted traffic light periods and speed regulations. A cooperative intelligent transportation system (cITS) can even divert traffic from originally intended routes to achieve abstract coordinated goals such as avoidance of congestion during peak traffic times.

\subsection{SCOPE}

On a technical level, communication between vehicles and the surrounding world can be implemented in many different ways. A general categorization differentiates between two categories: Centralized communication services that rely on dedicated infrastructure, for example via mobile phone services in cellular networks, and decentralized spontaneous communication among individual vehicles. The latter type is referred to as vehicular ad-hoc networking technology (VANET).

The domain of vehicular ad-hoc networking can be regarded as a specialization of the older mobile ad-hoc networking domain (MANET). However, the unique attributes of the vehicular context require dedicated solutions. A defining characteristic of vehicular ad-hoc networking is the high volatility of the network, due to the high relativevelocities of mobile nodes, and the necessity of low-latency communication with high-availability for safety-critical applications. These aspects lead to a prioritization of direct single-hop communication in vehicular ad-hoc networking, while classic mobile ad-hoc networking tends to focus on delay-tolerant networking techniques over multiple 
nodes. Chapter 3.1 will discuss in more detail the unique demands of vehicular ad-hoc networking and derive concrete requirements.

Multi-hop communication and communication with centralized services are foreseen to be possible over vehicular ad-hoc networks. Additionally, it is expected that vehicular networks might interoperate with non-mobile entities, such as traffic lights, or non-vehicular entities, such as pedestrians. A multitude of designators have been introduced to label and discuss the peculiarities of such sub-problems, using the pattern of vehicle-to- $X$ communication. For example vehicle-to-infrastructure, vehicle-to-cloud, vehicle-to-roadside, vehicle-to-pedestrian, etc. However, this dissertation focuses exclusively on the core vehicle-to-vehicle $(\mathrm{V} 2 \mathrm{~V})$ use-case of vehicular communication, as it provides the central cooperative awareness service that enables most other cITS services.

More specifically, the objective of this dissertation is to investigate the scalability of broadcast authentication in the domain of vehicle-tovehicle communication. Local broadcasts are the natural dissemination mechanism for wireless ad-hoc communication. Providing information concurrently to all receivers in a broadcast fashion is useful to minimize communications latency, which is highly desirable for safety-related services. In addition, as discussed above, the information also needs to be authenticated in order to deny potential attackers the possibility of participating in the communication. Again, chapter 3.I will discuss the specific requirements for broadcast authentication in vehicle-to-vehicle communication.

Scalability of an authentication technique in this context pertains to behavior under load, which can be caused by benign heavy usage or by malicious behavior. This aspect is commonly subsumed in security literature under the topic of "availability". Secure broadcast communication amongst vehicles is bounded in particular by the capacity of the communication channel and by the computational resources that are available in vehicles. Both aspects are investigated in this dissertation.

\subsection{RESEARCH OBJECTIVES}

The first objective of this dissertation is a review of security and privacy requirements of broadcast authentication in the context of vehicle-tovehicle communication. The goal is to not just reiterate a set of abstract requirements but to derive quantifiable constraints on resource usage. The focus will be set on resources that will bound $\mathrm{V}_{2} \mathrm{~V}$ broadcast authentication solutions in particular. These will be the communication channel capacity, computational resources and aspects relating to privacy. As a follow-up to this first goal, it should be possible to investigate if the solution space for $\mathrm{V}_{2} \mathrm{~V}$ broadcast authentication is changed or reaffirmed. 
- Rı: Can we refine and quantify security and privacy requirements for $\mathrm{V} 2 \mathrm{~V}$ communication, especially with respect to scalability issues for broadcast authentication such as computational processing, network communication resources, and achievable privacy?

- R2: How can more precise bounds and refined requirements open-up (or narrow-down) the solution space for broadcast authentication in $\mathrm{V}_{2} \mathrm{~V}$ communication?

Going beyond the usual assumptions of security in vehicular communication, we want to broaden the perception of attacker models in cITS. Broadcast authentication is a powerful solution to the problem of denying unauthorized third-parties the possibility of participating in vehicular communication. However, this is a very network centric view of cITS. A reassessment of possible attack vectors and attacker capabilities should deliver a better perspective on likely attacks. This is a necessary exercise in understanding the role and limitations of broadcast authentication in the overall context of secure cITS.

- R3: What are realistic attacker capabilities and attacker models in cITS and what are the implications for counter measures.

The research questions $R_{1}, R_{2}$, and $R_{3}$ are covered in Part II of this dissertation. Part III is dedicated to the investigation of hardwareassisted solutions to reduce the cost and overhead of pervasive security and privacy of $\mathrm{V}_{2} \mathrm{~V}$ broadcast authentication. This hardware-assistance will take the form of a Hardware Security Module (HSM). Two functions provided by Hardware Security Modules will be investigated: secure storage and acceleration of cryptographic algorithms.

Privacy-preserving broadcast authentication can require secure storage of large amounts of key material. We will assess the utility of a Physically Unclonable Function (PUF) as a solution to the provision and protection of such key material.

The second research question concerns the acceleration of cryptographic primitives that are relevant for $\mathrm{V}_{2} \mathrm{~V}$ broadcast authentication. The investigation focuses on the collection and evaluation of performance-test results, in order to establish if cryptographic acceleration hardware is a hard requirement for the cost-effective deployment of secure vehicular communication. A set of detailed measurements and evaluations of the performance and overhead of software-based and hardware-assisted security solutions for vehicular communication is given. These measurement contribute realistic baseline information about the necessity of hardware-assisted security for the first generation of $\mathrm{V} 2 \mathrm{~V}$ deployments.

- Hi: How could the availability of a Physically Unclonable Function (PUF) support scalable security and privacy in $\mathrm{V}_{2} \mathrm{~V}$ ? 
- H2: Is hardware acceleration of cryptographic primitives necessary for pervasive secure broadcast authentication in $\mathrm{V}_{2} \mathrm{~V}$ ?

Finally, in Part IV, the management of certificates is identified as a potential source of large savings of communication overhead. Broadcast authentication with certificates requires the exchange of certificates only at the first encounter of vehicles. Due to the size of such certificates it is useful to consider certificate omissions schemes that minimize the transfer of this data.

- $C_{1}$ : What is the essential trade-off of certificate omission within $\mathrm{V}_{2} \mathrm{~V}$ communication and how can it be measured?

- C2: Do existing proposals for certificate omission have problems with scalability?

- $C_{3}$ : Is maximum omission of certificates the most efficient strategy?

- $\mathrm{C}_{4}$ : Can a congestion-based certificate omission scheme deliver better performance than previous proposals?

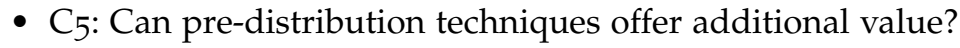

Several research question need to be answered to provide an evaluation of certificate omission schemes for scalable certificate distribution for $\mathrm{V}_{2} \mathrm{~V}$ broadcast authentication. Our objectives range from the identification of relevant performance attributes, through the measurement of efficient trade-offs, to the evaluation of a novel congestion-based omission scheme. The last research question will then go beyond certificate omission and investigate the potential of a novel pre-distribution technique as an enhancement for $\mathrm{V}_{2} \mathrm{~V}$ certificate management. 
Part II

REASSESSMENT OF REQUIREMENTS 



\section{2}

OVERVIEW

Situational awareness about the surrounding environment is the core enabling feature of intelligent and automated vehicles. The acquisition and perception of the environment through sensor data is the first step in building situational awareness. Consequently the quality and range of sensor data input is a major bound of the achievable performance of intelligent and automated vehicles.

Independent of the type of sensor, we can identify two sources of sensor data:

- Locally generated sensor data

- Cooperatively shared sensor data

All types of sensors have a limited range. Sharing sensor data with other vehicles makes it possible to greatly extend the range of sensor coverage. This represents a large added value for the quality of situational awareness.

This simple principle is at the core of cooperative driving and is expected to form an essential component in providing long range situational awareness for intelligent vehicles and automated driving. Since vehicles are intrinsically mobile all communication will occur through wireless communication. Various options are available for the exchange of information through wireless networking, however the peculiarities of vehicular communication narrow the solution space. Vehicular communication is

- ad hoc, due to the volatility of highly mobile communication partners

- low latency, due to the involvement of safety-of-life decision making

- infra-structureless, due to the impracticality of deploying $100 \%$ coverage

- broadcast oriented, due to the nature of wireless transceiving 
A dedicated solution for the needs of vehicular ad hoc networking has been proposed and successfully went through international standardization processes. The result is the IEEE 802.11p profile and the ETSI ITS G5 adaptation of the IEEE 802.11 wireless communication standard, which is expected to be used as the basis for vehicular networking at least in north american and european markets. The use of cellular data communication networks and IP based communication are possible alternative options, however this is out of scope for this work.

The specification of 802.11p notably includes feature to enable robust low latency exchange of information. The main difference to standard IEEE 802.11 is the Outside-the-Context-of-BSS (OCB) operation mode, which allows the use of the allocated frequency spectrum without prior association into a basic service set (BSS). In this mode it is possible to use the 802.11 wireless protocol stack in an almost stateless broadcast mode, which better suits the low latency ad hoc communication requirements of vehicular communication.

However, the use of 802.11 in a broadcast mode such as OCB implies that none of the usual security features of 802.11 are applicable. Omitting security in a system that will ultimately be used in the context of decision making, with the possibility to impact the safety of life of human users, is not acceptable. It could have catastrophic consequences for the safety of a vehicle and its passengers, if an attacker could successfully influence a vehicles perception of its environment through manipulated sensor data. The security of vehicular communication needs a solution that takes into account its peculiar requirements in order to enable trust in the wirelessly exchanges sensor data.

In the following chapter we discuss realistic goals and constraints of security in this specific context of vehicular communication. We begin with a reassessment of security goals and the consequences for the solution space of cryptographic primitives for broadcast authentication. Then we review privacy goals in $\mathrm{V} 2 \mathrm{~V}$ communication, with a special focus on what is actually achievable in this domain. A short survey of recent results in tracking efficiency allows us to identify unrealistic goals and approaches. Then we proceed to quantify concrete constraints for communication overhead and performance demands for security in vehicular communication. Awareness of these constraints can provide important guidance in discussions about the suitability of alternative signature schemes and about the need for the provisioning of suitable computational resources.

Finally, we dedicate a chapter to a review of attacker assumptions in the bigger context of cITS. Broadcast authentication of vehicle-to-vehicle data transmissions is a key instrument to protect the trustworthiness of cooperatively shared information. But it is important to see this protection in the bigger context of the data lifecycle in cITS. We explore 
the potential of attacks at different stages of the data lifecycle and describe attack vectors and attacker capabilities that need to take into consideration for deployments of vehicular communication services. 



\section{GOALS AND CONSTRAINTS}

\subsection{SECURITY REQUIREMENTS}

The fundamental goal of broadcast authentication is to allow the receivers of a message to verify the authenticity of a message. In practical terms this pertains to the integrity of the message and the identity of the sender. The sender of a broadcast might know nothing about the receivers and there may be more than one receiver. A requirement for the authenticity of messages also naturally implies the need for protection of the integrity of that message. The expected operational lifetime of a security solution dictates the amount of cryptographic strength that is required to provide sufficient protection against brute force attacks. Studies of vehicle lifetime investigate the survival and disposal rates of vehicles up to 30 years and list median lifetimes between 16.9 and 28.o years [27]. To provide security for timespans up to 30 years we follow [30] to require effective brute force resistance equivalent to symmetric key lengths of 128 bit. Protection against advances in quantum computing are considered optional.

The primary goal of authenticity and integrity in vehicular networking is to ensure that communication is not manipulated and only takes place between certified vehicles. However, the assurance that an entity represents a vehicle is not sufficient for secure communication in vehicular networks. If vehicles get involved in accidents or if there is another need to show evidence of authentic messages to resolve a dispute, it is a requirement to have non-repudiation of received messages.

Additionally, a core service of vehicular communication is cooperative awareness, which is provided through regular broadcast that announce the position and trajectory of vehicles. To securely track vehicles over time we require short-term linkability of the broadcasts, which proves that a sender is identical between two messages. For privacy reasons it shall be possible for vehicles to nevertheless change between pseudonymous identities under specific circumstances. This allows to break long-term linkability while preserving short-term linkability. However, the possibility to use multiple identities shall not enable sybil attacks, which will require careful temporal scoping or 
proof-of-work schemes. Research into the details of when and how to change pseudonyms is ongoing [108] but out of scope for this work.

Confidentiality is not a universal requirement for secure vehicular communication. A large majority of broadcast applications in this context is intended to share information to the public. For special applications that require confidentiality it is possible to overlay additional protocols or to encrypt data payloads at the application level.

Finally, we require the availability of secure broadcast message processing under the assumption of a fully adversarial network [88]. While the frequencies allocated for vehicular communication might remain regulated, the use of a wireless communication medium necessitates the consideration of such powerful attackers. We note in particular that the network model of $\mathrm{V}_{2} \mathrm{~V}$ authentication does not guarantee uninterrupted availability of trusted third parties. Even a reverse communication path from the receiver of a message back to the sender is not a realistic assumption due to hidden station effects in wireless networks.

To summarize the security requirements for secure broadcasts in vehicular communication:

- (Broadcast) Authenticity

- (Short-term) Linkability

- Non-repudiation

- Availability

As pointed out in [104] and [88], Boneh et al. [15] showed that short and collusion resistant broadcast message authentication must rely on digital signatures. Short length in this context means that the length of the authenticator should be independent of the number of receivers. More efficient schemes would need to rely on additional assumptions such as time synchronization[104]. However, we note in particular that, if non-repudiation is required, digital signatures or equivalent constructions are the only currently known suitable solution.

\subsection{ACHIEVABLE PRIVACY}

Privacy for passengers of cooperative vehicles was identified as a requirement for market acceptance quite early in the process of developing vehicular communication infrastructure. Golle et al. [56] discuss privacy in the context of authenticated vehicular position beacons as an aspect of distinguishability and mention changes of key material as a counter measure against long lived identification. The SEcure VEhicular COMmunication project (SeVeCom) [97] collected relevant attacker scenarios and proposed a formalized pseudonym system as a useful 
approach to provide privacy in vehicular contexts. The use of pseudonyms does not imply anonymity, because short-term identities are still attached to vehicles to ensure accountability and non-repudiation. The key requirement for the effectiveness of pseudonyms is their unlinkability for attackers, while authorities may have the ability to resolve pseudonymous identities to the owner of a vehicle. Depending on this effectiveness, this kind of pseudonymous authentication scheme can offer revocable privacy [123] or even reasonable levels of true anonymity [54]

However, important details remain underspecified and subject to research. The biggest open question concerns the strategy for pseudonym changes, which has a large influence on the effectiveness of pseudonyms. Previous research efforts have already highlighted challenges of performing effective pseudonym changes. According to these efforts it requires drastic measures, such as silent periods $[62,121]$ or context sensitive collaborative operations in mix-zones $[6,52]$ to ensure meaningful k-anonymity. Only recently have researchers started to investigate the impact of pseudonym change strategies on application level service quality [85]. Nevertheless the full consequences and practicability of pseudonym change strategies in realistic environments are still not well understood.

In field-operational-tests (FOTs) and publications surrounding the relevant standardization efforts we commonly find assumptions of periodic pseudonym change strategies. In the context of early solutions in the context of IEEE 1609.2 we find estimates of pseudonym changes periods of around 5 minutes [33, 138].

A PKI model proposed within the CAR 2 CAR Communication Consortium has influenced recent FOTs by generally assuming the availability of certificates with multiple overlapping validity periods. This allows flexible change strategies [10]. Yet the proposal avoids specific recommendations, instead calling for standardization of boundaries without providing further suggestions:

"The pseudonym change strategy and frequency is out of scope of this work, since we consider it as a feature specific to manufacturers. For security and effectiveness reasons, we only advocate to standardize boundaries of maximum and minimum frequency."

In academic literature we find recommendations of periodic pseudonym change strategies with time periods between 1 minute [115] and 10 minutes [58]. A recent survey of pseudonymity schemes for vehicular networks [108] covers a multitude of strategies, classifying them into 6 categories

1. Fixed time change (periodic)

2. Random change 
3. Silent period between change

4. Vehicle-centric

5. Density-based

6. Collaborative (synchronous) change

A ranking of performance characteristics is not included in the aforementioned survey due to lack of universal privacy impacts metrics and lack of suitable quantifications of side-effects on safety and scalability. These problems are identified as future work.

The potential for Sybil attacks has been identified in previous works related to security and privacy in vehicular networks [122, 147], which is a reason to strictly limit the validity of pseudonymous certificates. Recommendations for deployments of pseudonymous certificates suggest lifetimes of around five minutes [33]. However, such configurations would prevent pseudonym change strategies that rely on unpredictable context sensitive and/or collaborative pseudonym change strategies. Any unpredictable pseudonym change strategy requires the availability of multiple valid pseudonyms. Proof-of-work systems might counter simple sybil attack scenarios, though fundamentally the risk of sybil attacks remains.

Recent research by Lefevre and Petit [85] has highlighted the severe impact of silent periods $[62,121]$ as part of a pseudonym change strategy on service quality of Intersection Collision Avoidance (ICA) applications. This observation is unlikely to be limited to ICA applications. Cooperative awareness is the fundamental building block of many safety applications in vehicular networks, such as ICA. An unfortunately timed pseudonym change could break the stability of cooperative awareness. The basic position beacons that all vehicles are expected to broadcast to announce their position and trajectory are sometimes called Cooperative Awareness Messages (CAM) [39]. These are mandatory messages and the awareness of the exact position of surrounding vehicles is a key enabler for most safety applications. The need for awareness of surrounding entities is a fundamental requirement. Privacy preservation efforts must not interfere with this requirement. If a fully anonymous communication protocol was available, it would not be an applicable solution for vehicular communication networks. This is because it would make entities untrackable even in close proximity, thus breaking the correctness of the awareness of surrounding vehicles. Local trackability is the foundation of cooperative safety.

As pointed out by Lefevre et al. [85], if pseudonym changes include long silent periods, it would become untenable to build services that provide safety critical services. It appears reasonable to only allow silent periods in situations without any safety relevant interactions with other vehicles. However, it is not predictable if and how frequently 
such situations will occur. Furthermore, due to hidden station effects even the detection of such situations is unlikely to be reliable enough for consideration in combination with safety critical applications.

Mix Zones [6] have been proposed as a way to collaboratively perform pseudonym changes. This technique can give a reasonable amount of expected k-anonymity even under the assumption that an attacker can observe the entire pseudonym change process. The Mix Zones concept achieves a considerable effectiveness in this scenario, however the attacker is considered to be a passive observer. The synchronization of pseudonym changes with other entities implies that privacy decisions depend on external input. Unavailability, inability or even malicious unwillingness to participate in a pseudonym change process might prevent vehicles from ever changing their pseudonyms. Additionally, the adherence to as combined silent period would be problematic for the above mentioned reasons. This also applies to encrypting messages instead of stopping to send messages, as proposed by Freudiger (CMIX) [52]. The potential inability of nearby vehicles to process messages would have a similarly negative effect on service quality, while high resolution tracking would still allow for tracking of even encrypted beacons.

It is useful to narrow the solution space by identifying what can realistically be achieved in practice. The primary boundary is the attacker model that defines the effectiveness of pseudonym changes. Any pseudonym change strategy is ineffective if an attacker can link different pseudonyms through simple observation. The main design criteria for effective pseudonym change strategies are assumptions about the attacker coverage and the consequences thereof.

A second boundary is imposed on pseudonym change strategies by the fact that side-effects on safety and scalability of $\mathrm{V}_{2} \mathrm{~V}$ communication should not affect safety-of-life services in negative ways. We assume that concerns about service quality for safety-of-life applications in vehicular communication networks will take precedence over privacy considerations.

Investigating achievable goals against attackers and considering negative impact on service quality as unacceptable, provides tight bounds for the solution space. Additionally, some techniques that appear detrimental to unlinkability become acceptable, once fundamental constraints of attacker uncertainty and application service quality become apparent.

\subsubsection{Local observer}

Intuitively it appears desirable to change pseudonyms frequently and unlinkably. Nevertheless, studies of data plausibility checks [9] have demonstrated that - even under the assumption of perfect unlinkability 
- protection against tracking by entities in local communication range is futile. This is due to the fact that vehicles continuously broadcast their precise position and trajectories. These announcements are known as Basic Safety Messages (BSM) or Cooperative awareness Messages (CAM) and represent a core feature of vehicular communication. In fact, it would be counterproductive to aim for location privacy against vehicles in local communication range, because achieving local tracking is the fundamental goal of these broadcasts. It is a feature of vehicular communication to create authenticated linkability for local entities.

\subsubsection{Global observer}

Protection against a global all-seeing attacker is practically impossible, for the same reasons. An attacker with universal coverage can create linkability through observations of all local BSM and CAM messages. Even under the absence of any other identifiers, interpretation of the positions and trajectories will enable effective tracking for global observers [139].

Silent periods and mix zones are effective techniques to create uncertainty even for a global all-seeing observer. However, the use of silent periods is not acceptable while a vehicle is participating in traffic. The introduction of silent periods can degrade the quality of service of important safety-of-life applications, such as intersection collision avoidance applications [85].

Proposals exist to introduce cryptographic silent zones [52], which can protect against passive global observers. Nevertheless, there will always be a degradation of service while enrolling newly arriving vehicles into cryptographic silent zones. All active entities in a cryptographic silent zone need to be supplied with valid cryptographic key material to be able to decrypt position beacons of neighboring vehicles. Furthermore, an attacker can participate actively in a mix zone to receive relevant key material. Additional assumptions about the availability of supporting infrastructure, such as road side units (RSU), limit the practical applicability of cryptographic mix zones.

\subsubsection{Medium observer}

The pervious paragraphs indicate that pseudonym change strategies cannot and should not be effective against local attackers. Furthermore, techniques to provide unlinkability against global observers, such as silent zones, would have a negative impact on service quality. This negative impact might be small, but will be unacceptable in the context of safety-of-life applications. 
This leaves attackers with gaps in coverage as the only model that pseudonym changes can reasonably effective against. Such protection against medium sized observers is still useful. A large class of potential attackers is likely to have such non-perfect coverage. It is a realistic goal to maximize uncertainty for this kind of attacker and maximize the cost of effective location tracking. A comparable model is the protection of metadata through onion routing, which can effectively only increase the resources required for successful surveillance while not guaranteeing perfect protection of metadata against attackers with very broad or strategic observation capabilities over a network.

The main consequence of this observation is that there is no reason to hide the occurrence of a pseudonym change from any observers within communication range. Only real gaps in the coverage create uncertainty for an observer. This has implications for the selection of pseudonym change strategies, which are the primary privacy enabling technique in $\mathrm{V}_{2} \mathrm{~V}$ broadcast authentication. Since it is futile to hide pseudonym changes, it becomes more acceptable to consider approaches that propose to announce pseudonym changes. Announcements are useful to coordinate pseudonym changes in order to maximize the number of participants, which is them more likely to create actual uncertainty for observers with gaps in their surveillance coverage. Additionally, in chapter 14 we will introduce a certificate pre-distribution technique, which uses announcements of upcoming pseudonym changes to distribute certificate material more intelligently. Such a technique for temporal pre-distribution of certificate material can reduce the occurrence of unverifiable packets and thus makes pseudonym changes in moving traffic much safer.

\subsection{QUANTIFYING BANDWIDTH CONSTRAINTS}

The physical limitations of the available communication channel imposed natural upper limits for the bandwidth consumption that is tolerable for secure $\mathrm{V} 2 \mathrm{~V}$ communication. The hard limit for the broadcast packet size is defined by the Maximum Transmission Unit (MTU) of the underlying wireless data link layer. A set of modifications and extensions to the 802.11 wireless communication standard known as 802.11p have been introduced specifically for vehicular communication and are expected to be the medium of $\mathrm{V}_{2} \mathrm{~V}$ communication. As specified in the latest version of this standard [140] we assume a reference MAC layer service data unit (MSDU) of 2304 octets. Safety critical applications of $\mathrm{V}_{2} \mathrm{~V}$ communication require low latency processing, therefore we do not consider fragmentation or aggregation features on the data link layer. The ETSI ITS-G5A and ITS-G5B profiles [36] of 802.11 even explicitly disallow fragmentation at the MAC layer. 
Thus, 2304 bytes is a hard upper limit for the size of secure vehicular broadcasts.

It is intuitively clear that the size of payloads has direct implications for the quality of service in the wireless channel. Initial investigations [67] of 802.11p channel models suggested upper bounds of 500 bytes or 100 bytes for PHY layer service data units (PSDU) to ensure packet error rates (PER) below 10\% in typical scenarios. However, recent measurements [68] show PERs barely affected by PSDU length up to 2000 bytes due to advances in the implementation of channel tracking algorithms. Nevertheless, as the number of senders in communication range increases, the likelihood of waiting periods and packet collisions in the wireless channel increases. While techniques such as Decentralized Congestion Control [40] can reduce the impact of vehicular traffic congestion on wireless channel congestion, it remains important to minimize the size of payloads. The amount of data that vehicles exchange over the wireless channel at a given periodicity has a direct influence on the overall bandwidth consumption and thus on the likelihood of packet collisions. Consequently we consider the overall size of signatures and certificates as additions to the payloads for secure communication to be an important metric to evaluate the quality of proposed protocols.

The sizes of payloads in $\mathrm{V} 2 \mathrm{~V}$ communication are highly variable, which makes it difficult to give further boundaries on the size of security material. In the ETSI ITS architecture it is expected that all $\mathrm{V} 2 \mathrm{~V}$ communication is wrapped in GeoNet headers, which manage various aspects of routing as well as security. A requirement for the maximum transmission unit of an access layer $\left(M T U_{A L}\right)$ is specified in the respective standard document, with the aim to enable IP packet forwarding over GeoNetworking [38] as:

$$
\mathrm{MTU}_{\mathrm{AL}} \geqslant 1280+\mathrm{GEO}_{\max }+\mathrm{GEOSEC}_{\max }
$$

The stated expectation in the standard document assumes that common access layer implementations should support payloads of at least 2000 bytes. It is further expected that the payload capacity is large enough to encapsulate not just minimum size IPv6 payloads of 1280 bytes but also typical Ethernet payloads of 1500 bytes. Combined with a GeoNet header of up to 88 bytes, as specified in [37] these assumptions yield a maximum size for the GeoNet security components of 412 bytes:

$\mathrm{GEOSEC}_{\max } \leqslant \mathrm{MTU}_{\mathrm{AL}}-\mathrm{MTU}_{\mathrm{ETH}}-\mathrm{GEO}_{\max }=2000-1500-88=412$ 
Similar bounds can be found in the IEEE 1609 family of standards. The size of security components is only specified with respect to the concrete selection of cryptographic primitives in IEEE 1609.2. However, in the final report of the VSC-A project [1], which served as the guiding input for the IEEE 1609.2 standardization process, we do find a more open evaluation of sizes of security components. Here it is required that certificates should not exceed 300 bytes. An implementation using digital signatures is described as additionally using 70 bytes of over-theair overhead for secure messages, 6 bytes for a timestamp and 64 bytes for a digital signature. Thus, we get 370 bytes overall for the security components. Alternative solutions besides message authentication with digital signatures are evaluated as well. The largest message types among these alternatives are called Piggyback Certificate-Date packet. These consist of aggregations of Message Authentication Codes (MAC), Hash values $(\mathrm{H})$, Certificates (Cert), Time schedules (T), Signatures (Sig), and Time Stamps (TS). Assuming that the size of certificates is allowed to reach up to 300 bytes and accepting the suggested default values for all other fields we get:

$$
|\mathrm{MAC}|+2 *|\mathrm{H}|+|\mathrm{Cert}|+|\mathrm{T}|+|\operatorname{Sig}|+|\mathrm{TS}|=12+2 * 12+300+6+64+6
$$

We arrive at 412 bytes as an upper limit for the tests of suitable broadcast authentication schemes that were evaluated for inclusion in 1609.2. This matches nicely with the size limitation for security material with the ETSI ITS GeoNet specification. For the purposes of this survey we assume that 412 bytes is the upper limit for integration of security components into the established protocol stack for $\mathrm{V} 2 \mathrm{~V}$ communication. When we evaluate security components we always refer, as much as the protocol permits, to fully self contained material that enables the security validation of the transferred message. For the purposes of certificates we will assume a trust chain of length one, implying that a certificate will consist of 1 public key and 1 signature. We do not explicitly take into account the addition of headers and meta information such as capability descriptions that might be encoded in certificates. Fine grained credentials to attest permission to request special treatment and elevated rights in VANET applications can be encoded in certificates, but can also be realized through application specific means within the payloads of broadcasted messages.

We conclude by listing two requirement levels for the size of broadcast authentication material in the vehicular networking context:

- $\leqslant 2304$ byte to fit in the MSDU of IEEE 802.11p / ETSI G5A

- $\leqslant 412$ byte for integration in ETSI ITS and IEEE 1609 architectures 


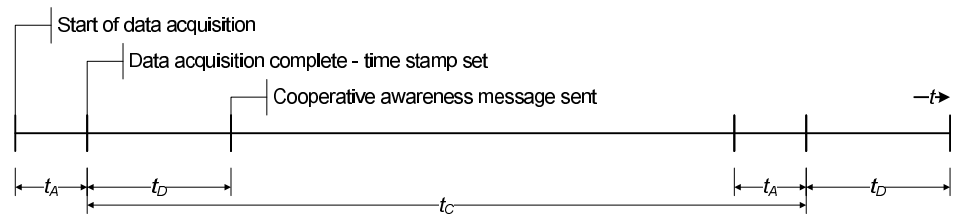

Figure 3.1: Time requirements for CAM generation and CAM processing according to ETSI ITS [39]

These constraints are met by the existing security designs from ETSI and IEEE, using cryptographic primitives based on elliptic curves. Any consideration of alternative cryptographic primitives, for example to provide resistance against attack by post quantum computers, need to fit within these constraints.

\subsection{QUANTIFYING PERFORMANCE CONSTRAINTS}

Vehicles can drive at high velocities, requiring fast reaction times from operators and driver assistance systems. Safety application in vehicular networks inherit this requirement and consequently the security services have to support these requirements. Faster processing times are of course always better, especially for event notification. But we can identify a set of timing constraints for message processing. Especially in the context of cooperative awareness services in the basic set of safety applications we find that information can become stale if it is delayed. Standardization efforts in IEEE/SAE and ETSI ITS have settled on $10 \mathrm{~Hz}$ as the target frequency for periodic cooperative awareness updates in addition to event notification messages. The research project PRESERVE uses scenarios from the simTD field operations trial project to estimate a load of up to 15 outgoing packets per second. A throughput of 15 messages per second implies 66 milliseconds as an intuitive limit on the time to generate authentication data. Delaying and queueing of messages to enable batch processing of data is not a useful option for the delivery of continuous update messages. Also some mutli-hop forwarding algorithms, notably Contention Based Forwarding which is included in the ETSI ITS architecture, dependent on constant processing times of outgoing messages.

The ETSI specifications for Cooperate Awareness Messages[39] (CAM) describe the process flow of CAM generation and processing as shown in Figure 3.1. The available time interval for data acquisition is given as $t_{A} \leqslant 50 \mathrm{~ms}$, time for CAM message processing up to the completion of the delivery process is equally allowed to require $t_{D} \leqslant 50 \mathrm{~ms}$. All CAM data, including timestamps, must be finalized before the generation of authentication data can begin. Therefore, the generation 
of authentication data must be part of the time segment allocated to delivery of CAMs, $t_{D}$. The allocation of $50 \mathrm{~ms}$ for the delivery of CAMs is consumed by the passage through the security, networking \& transport, and medium access layers. The delivery time can vary due to queueing effects in networking \& transport as well as medium access layers, which are not predictably constant. Nevertheless it is expected that security processing is free to consume a majority of the allocated 5oms. This fits well within the above mentioned throughput based bound of $66 \mathrm{~ms}$. It is generally advisable to minimize the amount of time required for the generation of authentication material. The ITS architecture is not required to follow hard realtime constraints, so tolerances for variances in processing time should exist. However, service quality is naturally improved by minimizing the latency of message delivery in general.

For a broader discussion of design considerations that influenced the development of the IEEE 1609 architecture we again refer to the results of the VSC-A final report [1]. Here we find again a requirement to sustain a throughput of 10 authentication generations per second. Additionally, we find a much tighter recommendation for bounds on the latency requirements: "The combined time required to generate an authentication of an outgoing $\mathrm{V}_{2} \mathrm{~V}$ message and verify the authentication of an incoming $\mathrm{V} 2 \mathrm{~V}$ message should be less than $20 \mathrm{~ms} . . . "$. The closely related DSRC Implementors Guide for SAE J2735 [119], which specifies message definitions for IEEE 1609, features even stricter overall reception latency requirements. These are based on demands of applications for different scenarios and vary between $\geqslant 20 \mathrm{~ms}$ and $\leqslant 10 \mathrm{~ms}$. A security solution that is expected to fulfill the requirements of all applications is thus expected to process messages within 1oms. However, these requirements concern not just the generation of authentication material on the sender side but the whole end-to-end message delivery process under ideal wireless channel conditions. The limit of 1oms thus applies to the combination of signature creation on the sender side and signature verification on the receiver side.

For the reception side we find an additional recommendation in the VSC-A report [1], requiring the ability to process a 1000 authentication verifications per second for incoming messages. This would directly translate to a demand for the ability to complete 1 authentication verification in less than 1 millisecond. However, on the receiver side it might be possible to queue up messages from different senders for batch processing, to potentially get more efficiency in the verification process. The overall processing time must still meet the aforementioned latency requirements though.

The research project PRESERVE effectively suggested less than 1 millisecond of processing time in the security subsystem on the receiver side of vehicular communication in an initial assessment of 


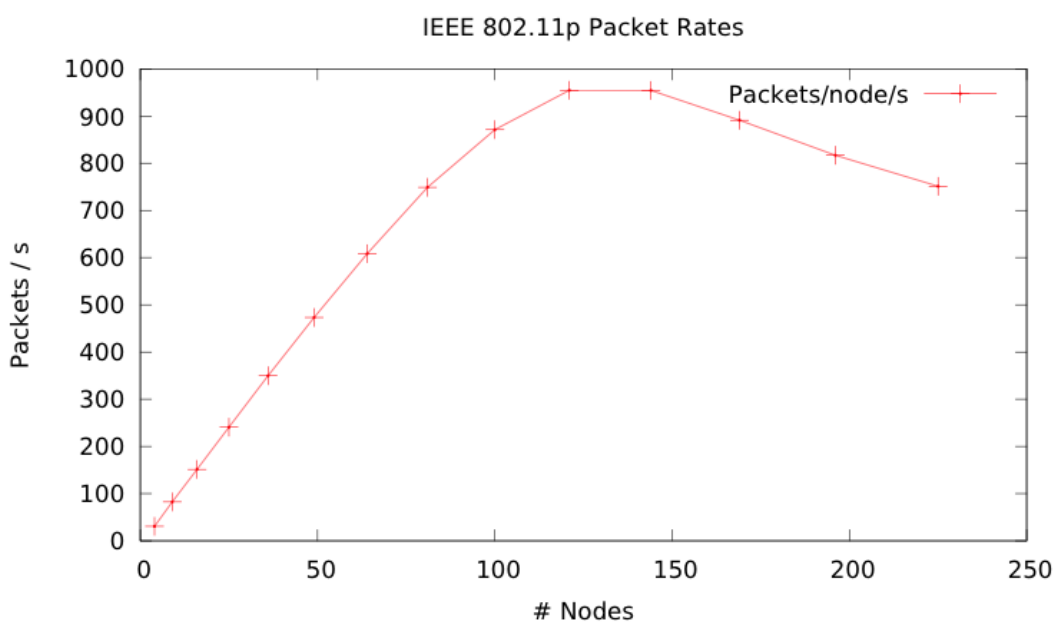

Figure 3.2: Maximum message throughput over a single 802.11p channel as measured in simulations by the PRESERVE project [133]

performance requirements [133]. The reasoning follows from a similar assumption of requiring the ability to sustain a throughput of 1000 secure incoming message per second, which was identified as the maximum throughput in a simulation study. This simulation result assumed a single $802.11 \mathrm{p} / \mathrm{G}_{5} \mathrm{~A}$ channel with increasing numbers of vehicles organized in a grid and all within mutual communication range. A peak throughput rate of close to 1000 secure messages per second was achieved with around 120 nodes communicating at $10 \mathrm{~Hz}$ each. This represents a hard limitation for incoming messages under these conditions, since the addition of further communication load resulted in lower message delivery rates. In the final results of the PRESERVE project [13] it is strongly recommend to exploit potential opportunities for parallel processing to reduce the cost of provisioning sufficiently powerful processing hardware. This implies that processing times could be higher than 1 milliseconds while still meeting the throughput requirement of 1000 messages per second. However, no further guidelines are provided for acceptable latency times.

To summarize, processing time for generation and verification of authentication data should be $<10 \mathrm{~ms}$ in to meet the strictest application specific requirements, as suggested by the DSRC Implementors Guide for SAE J2735. Additionally, we require the ability of verifying 1000 incoming messages per second. This second requirement represents maximum channel utilization and might be achieved by minimizing latency of verification to $\leqslant 1 \mathrm{~ms}$ or by parallel techniques, if the overall latency requirements are not violated. 
- $\leqslant$ Ioms latency for generation plus verification of secure messages

- $\geqslant 1000$ verifications of incoming secure messages per second

These performance requirements are challenging for contemporary embedded systems. In chapter 8 we will use these values to evaluate the suitability of hardware platforms that are used in vehicular communication trial projects. This will include an assessment of dedicated hardware acceleration designs for the relevant cryptographic algorithms. 



\section{4}

\section{ATTACKER MODELS}

\section{I DATA LIFECYCLE}

In the previous chapter we quantified performance and bandwidth requirements based on technical implementation constraints and application requirements. We also investigated abstract security requirements based on a rational analysis of security goals. And we discussed goals and constraints of privacy in vehicular communication and based this in large part on considerations of attacker capabilities. Attacker models are a very useful tool to assess the adequacy of counter measures with respect to the effectivity and coverage of counter measures against attacks.

In this section we focus on a broader perspective of attacker capabilities in the context of the specific application domain of vehicular networking. We present a data lifecycle model to identifying attack surfaces that enable attackers to influence or surveil the operation of a vehicular system. From this we derive attacker models that help to motivate further work that will be necessary beyond broadcast authentication, to insure the security of vehicular cITS solutions.

Figure 4.I shows our abstract model of data lifecycle stages, i.e. attack surfaces, in the vehicular communication domain. The sensor data is processed by the in-vehicle electronic control unit (ECU). The data is stored (data at rest) while unused or when the engine is off. Then, the data is sent by the communication unit to other entities in the $\mathrm{V}_{2} \mathrm{X}$ network (vehicle or infrastructure); this phase is called data in transit. This phase implicitly create additional meta-data in the form of externally observable information about the data in transit. Finally, when the data is received by a neighboring vehicle, the data is processed, stored, and used by the different layers of the communication stack.

The model presented in Figure 4.I is valid independently of any particular communication protocols, vehicle architectures or sensor types. This representation is reminiscent of signaling and control loop visualizations from cybernetics and systems theory. Though, we only consider a generic in-vehicle data processing entity instead of speculating about manifest representations of actuators or system outputs. 


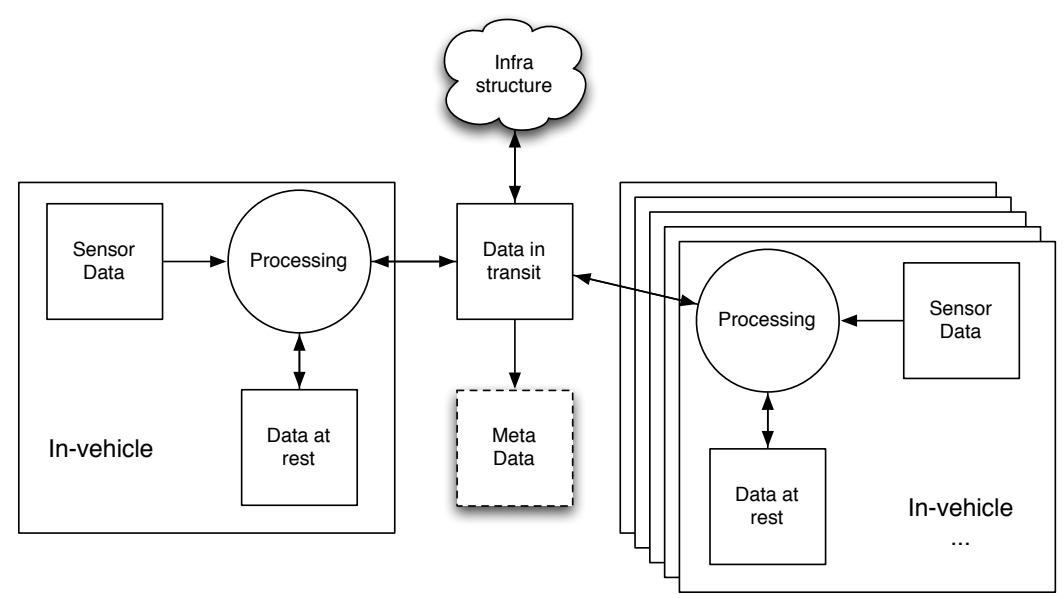

Figure 4.1: Abstract model of data lifecycle stages in smart vehicle domain

This way we can focus on the abstract possibilities of influencing or surveilling system behavior while the ultimate set of applications remains uncertain. In the context of attacker modeling this allows us to investigate attackers based on attack surfaces and derive attacker goals that have potentially been unknown or under-considered in approaches that start with attacker goals. Table 4.I shows how the attackers described in the following sections map to the stages in the data lifecycle shown in Figure 4.1.

Table 4.1: Attackers in the data lifecycle

\begin{tabular}{c|c|c|c} 
Data lifecycle & Sensor Confusion & Evil Mechanic & Communication \\
\hline \hline Acquisition & $\checkmark$ & & \\
\hline Processing & & $\checkmark$ & \\
\hline At rest & & $\checkmark$ & \\
\hline In transit & & & $\checkmark$ \\
\hline Metadata & & & $\checkmark$
\end{tabular}


From the attack surfaces defined in Section 4.1, we derive attacker models that allow more realistic reasoning about the achievable goals of attackers in vehicular ad hoc networking.

We assume that basic cryptographic primitives and implementations hold. Consequently we do not consider classic cryptanalytic attacks such as brute force attacks, side channel attacks, message forgery, or chosen message/plaintext/ciphertext attacks. Similarly, and with no loss of generality, we do not consider routing related attacks because day one applications will not be multihop [31]. Indeed, it is expected that the initial deployments of vehicular communication networks will be solely single-hop ad hoc communication ( $\mathrm{V}_{2} \mathrm{~V}$ and $\left.\mathrm{V}_{2} \mathrm{R}\right)$, and communication with infrastructure services will be through communication channels with routing services such as IP over LTE.

\subsubsection{Sensor Confusion}

The essence of telematic enhancements in vehicles is the augmentation of driver assistance through sensor data, ultimately up to the point of relieving the driver of the task of controlling a vehicle at all, leading to automated driving. The most common classic sensors are tachometer and odometers as well as driving related sensors e.g. for traction control. Through the addition of more advanced sensors, ranging from basic weather condition sensors to advanced positioning sensors, radarbased, or visual object and obstacle detection systems, vehicles gain increasingly better awareness about the environment. Attacking the initial data acquisition is a natural primary attack surface [106].

\subsubsection{Telematic illusions}

While some sensor data, e.g. about general weather conditions, can be provided by infrastructure services, the majority of safety relevant data will be collected autonomously by on-board sensors. On-board sensors are typically assumed to be fully trustworthy and provide a real-time depiction of the ground truth around a vehicle. This trust assumption however presents an attack surface based on uncontrolled input into the system with direct influence on its behavior. The cost and complexity of attacking sensors vary drastically, as does the effect of manipulating sensor data. A successful intervention on sensor data can range form blinding a sensor to creating false positive or false negative identification of objects in the environment. We call such phenomena telematic illusions and telematic blindness.

The attacker goals that we can directly derive from telematic illusions and telematic blindness are influences on local driver behavior, 
leading to erratic maneuvers, immobilization or even collisions. An example, could be misleading LIDAR systems using reflective or extremely light absorbing paint on a driveway. Similarly, misleading radar based systems using active echo generation could generate telematic illusions of obstacles, which could trigger sudden braking or evasive maneuvers. More primitive attacks could simply deafen radar based systems with overwhelming signals or blind visual systems with primitive off-the-shelf equipment such as laser pointers. For sensors to generate erratic measurements it can even be sufficient to simply manipulate the operating conditions to be outside the calibrated operational range. A common problem for many types of sensors would be the sensitivity to temperature of the environment. Heat-based active denial systems - originally designed to be non-lethal weapons against humans for crowd-control- could be used to remotely blind sensors and immobilize smart vehicles.

Countermeasures against this type of attack can be challenging to find, due to the limits of being exposed to measuring phenomena of the physical world. Filtering and sanitizing input from sensors is not a trivial matter, as it might be impossible for local sensors to differentiate between natural phenomena and artificial tampering of inputs. Sensible filtering and reasonable fail-safe behavior needs to take malicious tampering into account. Redundancy can provide a level of protection if individual sensors are subject to manipulation. Though, the conflict resolution of disagreeing sensor data readings is a hard problem as well. Tolerating benign as well as malicious byzantine faults is however well understood in distributed control systems. If the number of faulty sensor inputs remains below $2 / 3$, it can be expected that attacks can be tolerated. Interestingly, in systems with vehicular communication, it is possible for neighboring vehicles to collaborate on sensor data exchange to reduce the relative number of faulty sensor inputs [96]. On the other hand, and as described in Section 4.2.3, the cooperative solution can also be seen as a potential attack surface to introduce additional faulty sensor information.

\subsubsection{Time traveling}

A unique kind of sensor input that warrants special consideration is time. The main source of high precision time information in vehicles is expected to be a GNSS receiver such as GPS, which is also used to calculate global position information. Inaccuracies and limited availability of positioning information are a common problem, especially in urban city canyons or within buildings and tunnels. Telematic systems in smart vehicles are expected to use sensor data fusion to generate a local dynamic map for local orientation from multiple input sensors. The services relying on global positioning need to have a certain de- 
gree of tolerance. They also need to use signal smoothening and dead reckoning techniques to handle intermittent or inaccurate positioning information.

Time however is a resource that is usually considered to be precise and available to all vehicles at any time. Deviation from a globally accepted time source can lead to service disruptions. In the previous chapter we investigated timing requirements of message processing in vehicular networking. We found that messages that appear older than $50 \mathrm{~ms}$ can lead to an interpretation of a message as stale due to violation of freshness limits of data. A vehicle that is desynchronized from a globally accepted time source might reject inputs from other vehicles, or be ignored by receiving vehicles. More severe deviation of time on the order of days, months or years, can furthermore lead to interferences with the public key infrastructure (which provides the basic cryptographic services). Indeed, the available short-term certificates (i.e. pseudonyms) or even long-term certificate can be considered as expired. Hence, messages would be discarded incorrectly due to errors in the interpretation of validity periods. It might be reasonable to assume that misbehavior detection systems will classify vehicles with desynchronized time information as misbehaving, and evict them from vehicular communication systems by revoking their certificates.

None of the time sources that are readily available to mobile entities such as vehicles are cryptographically authenticated. These sources are terrestrial or satellite based signals such as DFC 77 or GPS. Deterrence of abuse and manipulation of these signals relies on wide scale monitoring of relevant frequencies by regulatory entities. Short and low powered local attacks in these frequency bands are hard to detect.

\subsubsection{Evil Mechanic}

After the initial intake of sensor data into a vehicular system, the data gets stored, processed and interpreted as information that influences the behavior of the system. Previous research efforts have primarily investigated security of information exchange among entities within vehicles [79, 135, 141]. Such work assumed a highly network oriented perspective, investigating only data protection on in-vehicle networks. However, physical access to in-vehicle resources has further implications on the security of the data. A more holistic perspective is necessary to consider additional relevant attack surfaces for an attacker with the capability to have physical access to vehicle components.

For the purpose of our discussion we assume that an attacker can gain short-term physical access to a vehicle. It is realistic to assume that an attacker can gain temporary possession of a vehicle, for example while the vehicle is parked or when a vehicle is left in garage for maintenance or repair. We do not precisely specify the time span of 
short-term physical access, but point out that protection against the owner of a vehicle is hardly possible or desirable. Protection against abuse by entities who only have short-term possession of a vehicle is however desirable. We call this model the evil mechanic in reminiscence to the evil maid attacker model [118], which is becoming popular in the cryptographic community.

\subsubsection{Exfiltration}

A classic attacker goal against a computer system is the exfiltration of sensitive data such as credentials or similarly interesting data. In the context of vehicular communication, this primarily concerns private key material, which is used to prove authenticity of vehicles. It is commonly assumed that private key material will be stored securely in a Hardware Security Module (HSM) [141]. The HSM stores data at rest in a secure manner. However, to reduce the attack surface against an attacker with privileged access to the system, it is also necessary to protect the private key material during the data processing, in this case during the generation of signatures. Therefore, the HSM should also cover all functionality that would normally require to have unencrypted private key material in random access memory (RAM) or registers. This effectively requires the HSM to be implemented as a compartmentalized trusted entity.

Private key material is not the only category of sensitive information in vehicles though. Logs and statistics that record usage data of the vehicle represent personally identifiable data, which also needs protection. A data encryption solution should help to protect data at rest, but without a human in the loop to provide key material such as passwords for the cryptographic operations. Not requiring human interaction does represent a challenge, since the system needs to be able to boot up and start operation fully autonomously, which implies that the system can access all relevant key material automatically. Full trusted computing, as seen in digital content protection systems such as game consoles or smartphones, would be required to achieve ideal goals of data protection. Such solutions would have to protect the entire system of information processing, starting from authentication booting and trusted execution up to resilience against hardware tampering and side channel attacks.

\subsubsection{Malicious data}

Even a fully trusted computing system will be susceptible to process inputs that are controlled by an attacker. This is closely related to sensor confusion as described in the previous section. An attacker with temporary possession of a vehicle can use all its trusted components to 
create malicious data or to avoid the creation of incriminating authenticated data. If telematic data will be considered trusted and permissible as non-repudiable evidence in courts, this will have significant effects. One way to exploit this attack surface could be the creation of valid messages such as position beacons with forged timestamps for later replay attacks. This could be used to incriminate the owner of a vehicle for actions that were not actually performed, for example in connection with insurance fraud. Fraud with maliciously authentic data could also be committed by the owner of the vehicle. Examples for such actions based on existing problems in the area of fraud with vehicles include odometer/tachograph manipulation, which document the usage of the vehicle for purposes of value determination for resale or for the control of driving time regulations and traffic violations. A simple way to implement this attack type could be the temporary detachment of trusted components in a vehicles telematic system or a transfer of these components into another vehicle. Strong monitoring and bindings of trusted components with a vehicle would be required to counter such attacks.

\subsubsection{Infiltration}

An attacker with temporary elevated access to a system is not only limited to reading or creating sensitive data. The addition or exchange of components with malicious features can allow an attacker to achieve persistent access to a system. This concerns hardware as well a software, although guaranteeing the trusted level of software on a trusted hardware platform is comparably less difficult. A controlled execution environment, for example as required for the protection against exfiltration of sensitive data, also enables controls of data execution using general computer security techniques such as instruction set randomization, code signing, taint analysis or misbehavior detection. Such intrusion detection systems however rely on the attribute of not being subverted themselves. An attacker with physical access can attack or circumvent and replace these systems as well.

Attacks against hardware, or even just the addition of new hardware that can perform malicious acts, is a very powerful attack surface. Covert installations of tracking devices are already a concern for victims of classic surveillance operations. Surveillance on smart vehicles with advanced telematic installation increases the impact of such techniques. Indeed, the attacker can gain a larger amount of data, for example by sniffing the content of communication on in-vehicle communication networks. Devices that can perform such acts do not require high levels of sophistication or monetary costs and can operate entirely passively from the perspective of regular on-board telematics. The addition of hardware that can selectively interrupt normal operation of vehicles 
services is similarly easy, cost effective and similarly hard to detect through automatic means. However, active insertion and manipulation of communication in in-vehicle networks can be mitigated by cryptographic means. Exchanges of whole components can similarly be mitigated by string cryptographic bindings of the components. Tamper resistant and tamper evident can furthermore make it more complex and costly for an attacker to subvert the security of a vehicle through the insertion of malicious hardware. All these measures do however increase costs and complexity not just for the attacker but also for the defender.

\subsubsection{Communication Attacks}

Cooperative driving and cooperative intelligent transportation system (ITS) in general are considered to be a key enabler of enhancements in traffic efficiency, ecology, and safety. The voluntary exchange of telematic data and cooperative behavior based on this information are expected to be beneficial for all participants of public vehicle transportation systems. For the security of individual participants, this willingness to cooperation can increase security but also open up new surfaces for intrusion.

\subsubsection{Fully Adversarial Networking}

The communicative aspect of cooperative driving is realized through wireless communication channels such as IEEE 802.11p and the related ETSI G5A standards. While the frequency band for use in vehicular communication are expected to be regulated, it is necessary to assume that the wireless channel is open to arbitrary use by adversarial entities. A requirement to deliver services under such attacker assumptions implies consideration of fault tolerance that influences the design of the overall communication patterns in this network. The two main methods for reliable operation in fully adversarial network conditions are cryptographic authentication of all messages to exclude unauthorized participation, and purely stateless message oriented communication patterns to avoid susceptibility to selective denial of service attacks. This aspect of inter-vehicle communication security is well understood and expected to be implemented by means of pervasive use of digital signatures of message oriented broadcasts [35].

\subsubsection{Medium Observer}

Surveillance on the information exchange in cooperative ITS is a problem that was identified as relevant and attracted some attention but remains largely unsolved. Cooperation implies the consenting sharing 
of information with potentially unknown neighbors without a requirement for confidentiality. Information such as position and trajectory of vehicles is willingly broadcasted to all other vehicles in communication range. This sharing of information enables cooperative awareness, which essentially is local tracking of neighboring vehicles. There is no way to prevent such local surveillance against mobile attackers which simply remains within communication range of a target vehicle, as this is the intended behavior of $\mathrm{V}_{2} \mathrm{X}$ technology. Even a complete avoidance of linkable identifiers cannot prevent tracking. Indeed, as demonstrated in $[69,134]$, plausibility checks of location claims using Kalman filters and Particle filters [9] are sufficient to enable short-term local tracking. As such, this local tracking of nearby vehicles represents an interesting lower bound on attacker capabilities.

The natural upper bound for an attacker is an all seeing observer with the ability to perform active attacks. A combination of mix zones and silent periods could thwart an all seeing passive attacker, but silent periods may not be realistic options in safety relevant live traffic scenarios [85]. A study about the effectiveness of an all seeing attacker using Multi Hypothesis Tracking (MHT) was performed by Wiedersheim et.al. [139] and demonstrated high levels of success for the attacker, even under noisy data and extremely frequent pseudonym changes.

Finally, we see that the pseudonym changes are ineffective against powerful all seeing observers and ineffective against small but mobile observers. The protection level against medium-size attackers is subject to further research. It is likely dependent on attacker mobility and the coverage of the relevant area. Covering for example intersections and considering knowledge of pseudonym change strategies should enable very effective tracking. The main path towards realistic location privacy protection is likely to be the investigation of pseudonym change strategies, as the low latency broadcast requirement of the vehicular communication context is not suitable for privacy protection through methods such as onion routing. 



\section{SUMMARY AND CONCLUSION}

The investigation in this chapter provide foundations for the evaluation of security in the specific context of vehicle-to-vehicle communication. We began with a discussion of security goals in the specific context the vehicular wireless communication environment. This discussion led to the identification of digital signature algorithms as the fundamental primitive for $\mathrm{V}_{2} \mathrm{~V}$ broadcast authentication. An subsequent further analysis of achievable privacy goals resulted in a classification of observer capabilities with consequences for the evaluation of pseudonym change strategies. These are the main privacy protection feature in the certificate based revocable privacy regime of $\mathrm{V}_{2} \mathrm{~V}$ broadcast communication. Once hiding pseudonym changes from observers in communication range is deemed pointless, it becomes more acceptable to consider synchronized pseudonym change scheme for more effective observer confusion. This also has relevance for a certificate pre-distribution technique, which we will introduce in chapter 14 as a method that can make pseudonym changes in moving traffic less susceptible to unintended packet loss.

We also calculate size constraints for security additions to $\mathrm{V} 2 \mathrm{~V}$ broadcast messages by reviewing properties of the wireless communication channel and associated headers and formats of communication protocols. The existing choices of cryptographic primitives remain within these constraints and we did not attempt to propose deviating from the common choice of elliptic curve based cryptography. However, future alternative options for digital signature primitives must fulfill these requirements, which is expected to be challenging, for example for cryptographic algorithms that provide protection against attacks from quantum computers. A set of more immediately important constraints was derived for permissible performance overheads of secure message processing. Adherence to latency and throughput requirement is an important problem for the provisioning of suitable hardware designs of $\mathrm{V}_{2} \mathrm{~V}$ communication equipment. The demands and limitations that were derived from application standardizations will be applied in chapter 8 for the evaluation of relevant performance and hardware acceleration options for the execution of cryptographic algorithms. 
Finally, we contextualize $\mathrm{V} 2 \mathrm{~V}$ broadcast authentication in a wider perspective on attack surfaces and attacker models on cITS. We apply a data lifecycle model to show the role of broadcast authentication in the protection of data in transit, which conversely indicates that other security solutions are necessary to protect data at different stages. Data storage, processing and acquisition all require protection to ensure trustworthiness of cooperative driving solutions. We derived a set of attacker models, goals, and capabilities in the vehicular domain to motivate future work in this domain. 


\section{Part III}

HARDWARE SOLUTIONS 



\section{6}

OVERVIEW

Secure vehicular communication is expected to be deployed to large numbers of vehicles. For large deployments, it becomes worthwhile to investigate the utility of dedicated hardware components. Tailored hardware assisted solutions for security are common practice in information technology in the form of Hardware Security Modules (HSM). Such modules can be stand-alone external computers, plug-in cards, or on-chip components. The core idea is to offload sensitive cryptographic functions to an HSM to achieve the following benefits:

- Secure execution of cryptographic algorithms

- Accelerated execution of cryptographic algorithms

- Secure generation of key material

- Secure storage of key material

The main security feature of dedicated HSMs is the isolation from a host computer environment. Generic host computers can perform a variety of useful tasks in response to external inputs. However, the universal possibilities of generic computers represent larger attack surfaces and are more likely to be subject to infiltration by attackers. The isolation of sensitive operations into a dedicated HSM quarantines the handling of sensitive information within an environment with minimal exposure to outside influences. The likelihood of extraction of sensitive information, such as key material, from an HSM should be drastically reduced by virtue of a highly reduced interface that does not feature support for extraction of key material. This isolation is effective against an attacker that is capable of attacking a host computer that controls the HSM.

A secure execution environment should also offer protection against an attacker trying to perform side channel attacks. These attacks aim to extract sensitive data by observing external phenomena that occur during the operation of computer hardware. Examples for such leaks include naturally occurring acoustic or electromagnetic radiation, but also include observable behavior that is triggered by maliciously induced faults in hardware components such as processor registers. 
The methods to prevent such attacks include the usage of hardware enclosures, that block emission of side channel information, and utilization of specialized implementations of cryptographic algorithms. Such implementations avoid externally observable differences in behavior under different fault conditions. However, such side-channel resistant implementations often require additional computational resources compared to implementations that are optimized for other goals.

Provisioning accelerated execution capacities to guarantee availability of security services is another reason to consider HSMs. Dedicated hardware simplifies allocation of computational resources because the exclusive use eliminates the need for scheduling algorithms to regulate access to shared resources. In some cases, it might not be possible or cost effective to guarantee sufficient performance levels without the use of dedicated hardware. In such cases HSMs can also serve as crypto accelerators. Supporting scalability for large SSL and VPN servers is a well known application for hardware accelerators for static cryptographic applications. Providing security in the vehicular communication domain requires the capability to perform large amounts of digital signature verifications. This could represent a similarly well defined application of cryptography, which could be accelerated cost efficiently through dedicated hardware.

Besides the aforementioned attributes of secure execution of cryptographic algorithms, the secure management of private key material represents a core service provided by HSMs. Private key material might enter an HSM, but should never leave an HSM thereafter. Though ideally the secure generation of key material should also occur within the HSM in order to guarantee that the material can never be exposed in an unprotected environment. To enable private key material to remain exclusively within the HSM for its whole lifecycle, requires the creation of private keys within the HSM. This requires the capability to collect cryptographically secure random numbers within the HSM. In an environment with the possibility to allocate dedicated hardware resources, this is commonly achieved through the availability of a hardware based True Random Number Generator (TRNG).

The secure storage of key material is closely related to the secure usage of keys in the context of the aforementioned isolated executions environment. The attacker model for secure execution considers attackers with online access to a system. This implies that the system is operational and an attacker can gain code execution capabilities or random access to memory segments. A requirement for secure storage is concerned with the protection of data at rest. It should be impossible for an attacker with access to the storage medium to extract sensitive material. Disk encryption solutions can protect access to storage media by requiring user credentials such as passwords. For HSMs, it is 
typically not foreseen to make the contents of a storage medium accessible to users, as it is expected to remain within the trusted execution environment of the HSM. The main focus is then to protect against offline attacks, where an attacker can physically remove the storage medium and can try to extract information independent of the host environment. Classic options to provide security against such attacks are encrypted storage and tamper resistant enclosures of storage media, which ultimately destroy the content of the media if an attacker tries to gain access bypassing the regular access restrictions.

In Section 7 we present a novel option for secure storage of large amounts of key material, as it is required for pseudonymous secure vehicular communication. The proposed solution uses Physically Unclonable Functions (PUF) to provide secure storage through the hardware intrinsic tamper resistance attribute PUFs. The PRESERVE project showed the feasibility of including PUF hardware as a component for a vehicular security subsystem by including a PUF design in its prototype HSM 6.1.

Also included in the prototype HSM of the PRESERVE project is a TRNG module for use in key generation and signature creation. This module is based on ring oscillators and is designed to fulfill at least the requirements for classification as PTG.I-RNG, as defined by the BSI [76]. Further discussions of TRNG designs and research directions for configurable logic devices can be found in $[49,50]$.

Attributes of secure execution against attackers with varying control over logical and physical host environments are subject of ongoing research in multiple disciplines. Among the tools to enhance execution security are formal methods in software verification [93, 132], consideration of timing aspects in cryptographic algorithm design [7], and hardware assisted protection of control flow integrity [78]. A full survey of the developments in this area is outside the scope of this work.

In Section 8 we discuss aspects of execution speed of suitable cryptographic algorithms on contemporary vehicular on-board computers in greater detail. We use hardware platforms that are used in automotive field operational tests to gain a reference for the expected performance attributes of cryptographic services in vehicular on-board units. We also show the results of software performance acceleration efforts and the directions of generic hardware enhancements in relation to these platforms. 


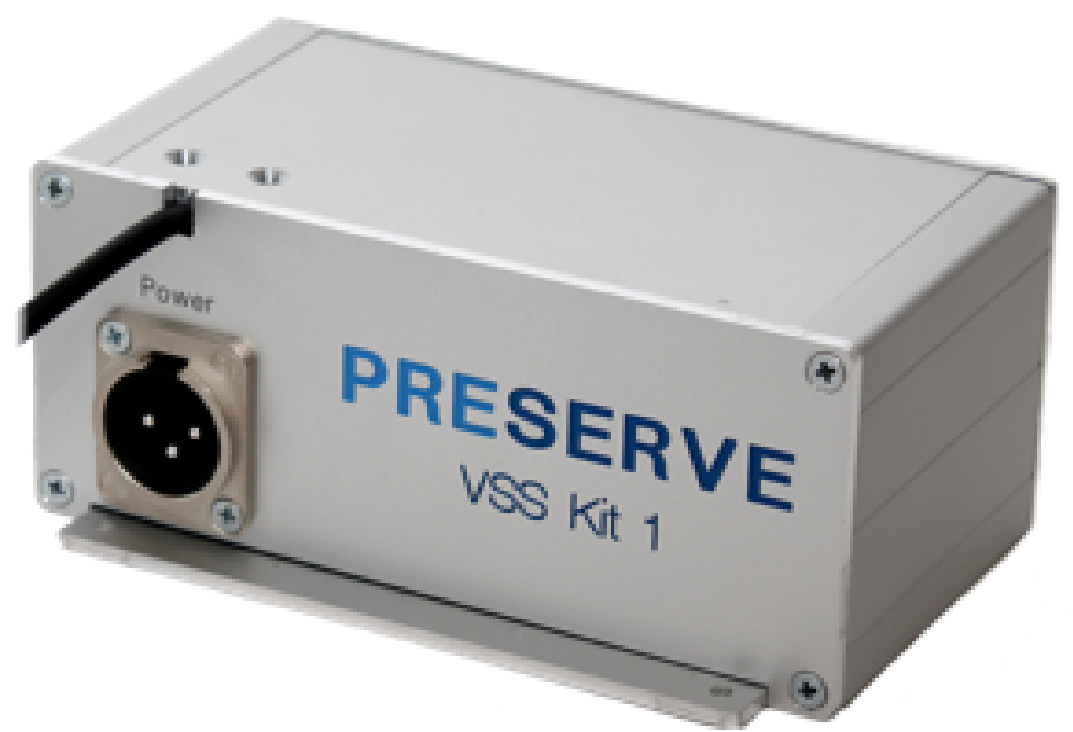

Figure 6.1: A prototype HSM device for use in secure vehicular communication, made by the european research project PRESERVE 
PSEUDONYM STORAGE IN PHYSICALLY

\section{UNCLONABLE FUNCTIONS}

Current standardization efforts, both in the U.S. and Europe, foresee that ITS will require the establishment of a Public Key Infrastructure (PKI), which manages trust and certificates in the ITS. The current set of standards [32, 34, 35] mandates the use of Elliptic Curve Digital Signature Algorithm (ECDSA) with P-256 elliptic curve for message authentication. A naïve implementation of authentication with digital signatures breaks user privacy as every receiver learns the identity of the sender. Therefore, a pseudonymous credential - short pseudonymshould be implemented in order to facilitate authentication and prevent direct vehicle identification. One single pseudonym is not enough to ensure a sufficient level of privacy. Instead, this pseudonym has to be changed frequently, and even then, a powerful attacker may be able to track vehicles [139]. Central questions are how many such pseudonyms a vehicle should store and how often it would have to contact the PKI for renewal. In general, a frequent connection to the PKI to renew pseudonyms cannot be guaranteed because large-scale coverage by road-side units (RSU) or cellular communication in every vehicle is considered unrealistic during early years of $\mathrm{V}_{2} \mathrm{X}$ deployment. Thus, the OBU has to store a potentially large set of pseudonyms to allow frequent change of pseudonyms in absence of backend connectivity. In a worst case, a vehicle would only be able to load new pseudonyms during annual inspections in a garage. Recent research estimates that an OBU is required to store 105,120 pseudonyms for one year with each pseudonym valid for five minutes [55].

Each of those pseudonyms consists of a public-private key pair and a corresponding certificate and especially the private key needs to be stored securely to not compromise security of the overall system. If an attacker acquires access to the secret keys stored in a vehicle, she could perform sybil attacks, spoofing attacks, and in general jeopardize the authentication and privacy of the victim. In consequence, it must be guaranteed that a private key is strictly secured during all events in its life cycle. This goal can be achieved by designing systems to securely create, manage and destroy (private) keys, maintaining an 
audit trail of every operation executed during their existence. Hardware Security Modules (HSMs) [131] are specifically designed to protect private keys. HSMs are specialized tamper-proof devices in which cryptographic functions and embedded software properly manage keys and control their life cycles. They are designed in such a way that if an unauthorised attempt to access them is made, this is considered an attempt to tamper and all critical internal parameters and keys are destroyed. The HSM features make them a crucial component in automotive platform security $[77,86]$.

However, HSMs are especially expensive if implemented on an FPGA [142], and a secure storage within an HSM adds complexity to the overall system. With ECDSA P-256 curve, the private keys of the one-year pseudonyms set proposed in [55] would require 256 bits $\times$ $105,120=3.2$ Mbytes of secure storage - not considering yet any overhead for data management. This requirement is too high as current solutions offer a maximum of 512 kbytes [2, 94]. Therefore, we aim at trading secure storage of cryptographic key material for regular storage (i.e. outside of the HSM).

\subsection{RELATED WORK}

A number of related publications have proposed ways to enhance the provisioning to and storage of pseudonyms in vehicles. We first describe the method of pseudonym provisioning based on PKI that underlies current standardization efforts. Secondly, we detail autonomous pseudonym provisioning techniques that aim at reducing the key material to be stored. Thirdly, we give an overview on how large amount of keys are stored in modern operating systems.

\subsubsection{PKI-based pseudonym provisioning}

The conservative PKI-based approach is limited to optimize the provisioning process of pseudonyms. This can be achieved by optimizing the trade-off between availability requirements and storage requirements. The allocation can happen on-demand, if a connection to a Certificate Authority (CA) is available, or by caching pre-allocated pseudonyms in local secure storage. In this context a pseudonym is defined as the pair of a public and private key as well as the related certificate issued by a trusted CA in the PKI.

To the best of our knowledge, detailed analysis of this allocation trade-off does not exist. However, a common assumption is to locally store a one year supply of pseudonyms inside vehicles [19, 55]. This allows to delay reloading of new pseudonyms until annual inspections, in case no other means of communication with the PKI backend exist. For resupplying pseudonyms, it is expected that vehicles will be able to 
intermittently use IP connectivity through road side units, residential WiFi or mobile phone networks.

A proposal to reduce the overhead of pseudonym certificates, specifically for the purpose of deployment in the vehicular communication domain, is the butterfly keys technique[138]. This method expands pseudonyms in a way that is very similar to the master key technique proposed in 7.3.3. However, butterfly keys are implemented as a lower level modification of elliptic curve cryptographic primitives, whereas the key derivation technique described in 7.3 .3 is more generically applicable.

\subsubsection{Autonomous pseudonym provisioning}

Some approaches allow vehicles to generate new pseudonyms themselves without interaction with a backend.

Group signature schemes [19] allow groups of vehicles to autonomously create key pairs for self-provisioned pseudonyms. These certificates validate the membership of a vehicle in a group, but do not reveal which member owns a particular pseudonym. Enrollment and removal of group members alter the group key material. This likely requires frequent rekeying and constant availability of the group manager, which can limit the appeal of this scheme. On the other hand, this scheme requires significantly less private key material to be stored. One valid set of long-term credentials for enrolling into an existing group or forming a new group is sufficient to bootstrap the system and create pseudonyms. Pseudonyms, including private keys, can be generated on demand and do not need to be stored beforehand.

Attribute-based authentication [23, 89, 143] allows a vehicle to generate pseudonyms entirely by itself, backed by a pre-shared cryptographic authorization attribute. A zero knowledge proof is performed between sender and receiver to verify the authenticity of a pseudonym without revealing any further identifying information about the signer. Similar to group signatures, only a set of long-term credentials for the zero knowledge proof is required to be stored. A vehicle can independently create pseudonyms, including private keys, on-demand.

The aforementioned pseudonym schemes have a distinctive set of advantages and disadvantages. Group-based signatures and attributebased authentication systems do not need to store large amounts of private keys for pseudonyms. Unfortunately, the utility of these systems is limited in practice by interactive protocols, reachability requirements for authorities, or slow bilinear pairing based cryptographic primitives. Moreover, as vehicles autonomously generate pseudonyms, the number of pseudonyms available to one vehicle per time cannot be limited. Hence, large-scale Sybil attacks become possible [144]. These limitations 
make the schemes unsuitable for practical applications contexts with low latency requirements, such as vehicular communication.

\subsubsection{Scalable secure key storage}

Solutions for the secure local storage of large amounts of key material exist in the form of password managers [116, 127], and more generically, in encrypted files [81] or filesystems [51]. These solutions store sets of keys and passwords in encrypted data stores, which are protected by a master secret, and additionally, by common operating system security features, such as access control lists.

Such solutions are effective with a human user in the loop to provide the master secret. In vehicular security it is not expected that a human user will provide a password or similar authentication data that could be used to unlock an encrypted data store. Instead, an On-Board Unit needs direct access to all the required data to boot up into a fully operational state. Effectively the decryption keys have to be stored together with the encrypted data. That implies that this type of solution cannot work as an self-sufficient secure storage system. Though, encryption of private keys can be part of a solution that employs another type of secure storage for the master secret. Such a solution is described in Section 7.3.2.

\subsection{SYSTEM MODEL}

In this section we describe the system model considered in this work. First, we explain Physical Unclonable Functions (PUFs) and related concepts. Then, we show how our solutions fit into the general OBU architecture. Finally, we describe the attacker model considered and discuss in Section 7.5 the level of protection offered by our solutions against such attackers.

\subsubsection{Physical Unclonable Functions}

A Physical Unclonable Function (PUF), as introduced in [101, 102], is a primitive that is bound to a physical system and extracts a pseudorandom bit string for key generation by mapping a set of challenges $C_{i}$ to a set of responses $R_{i}$. This challenge-response behavior is highly dependent on the physical properties of the device in which the PUF is contained or embedded. PUFs consist of two parts:

i) a physical part, which is an intractably complex physical system that is very difficult to clone. It inherits its unclonability from uncontrollable process variations during manufacturing. For PUFs on an Integrated Circuit (IC), these process variations are 
typically deep-sub-micron variations such as doping variations in transistors.

ii) an operational part, which corresponds to the function.

In order to turn the physical system into a function, a set of challenges $C_{i}$ (stimuli) has to be available to which the system responds with a set of sufficiently different responses $R_{i}$. The function can only be evaluated using the physical system and is unique for each physical instance because of process variations. Moreover, it is unpredictable even for an attacker with physical access.

PUF responses are noisy by nature. This means that two calls to a single PUF with the same challenge $c_{i}$ will output two different but closely related responses $r_{i}, r_{i}^{\prime}$. The measure of closeness can be defined via a distance function, e.g., the Hamming distance. This distance function should be small for responses from the same device and very large for PUF responses from different devices. Since the plain PUF responses are noisy, they cannot be used as a key. In order to derive reliable and uniform data from (imperfect) sources of randomness, such as a PUF, the concept of a fuzzy extractor [28] or helper data algorithm [87] was introduced. Thus, we obtain a master secret from the fuzzy extractor. This master secret can be the seed for a key generation scheme to derive public/private key pair(s) which can then be used as a pseudonym(s). Alternatively, the master secret can be used to first seed a key derivation scheme, which results in a larger amount of data that can then be used as seeds for key generation processes.

The formulation of abstract properties of PUF types as well as the development of PUF constructions are still a matter of active research [109]. In this work we use the terminology proposed by Rührmair et al. [117] and refer to Strong PUFs $^{1}$ and Weak PUFs ${ }^{2}$. To the best of our knowledge, no research has investigated the applicability of PUFs for storage of large numbers of private keys (or keypairs) as required by the $\mathrm{V}_{2} \mathrm{X}$ pseudonym scenario.

\subsubsection{On-Board Unit Architecture}

Figure 7.I shows the current ETSI reference architecture of an On-Board Unit (named "ITS Station" in the standard). It illustrates the different layers and particularly the security layer. A Hardware Security Module (HSM) is used within this architecture. As Figure 7.I is an abstract view of an OBU, and thus, does not represent the hardware, Figure 7.2 shows a simplified hardware architecture. An OBU includes CPU, host memory (RAM), regular storage, and an HSM. For simplicity, we represent an HSM that only includes a true random number generator

1 Labeled as "minimum readout time" PUF (MRT-PUF) [109]

2 Also known as Physical Obfuscated Keys (POKs) 


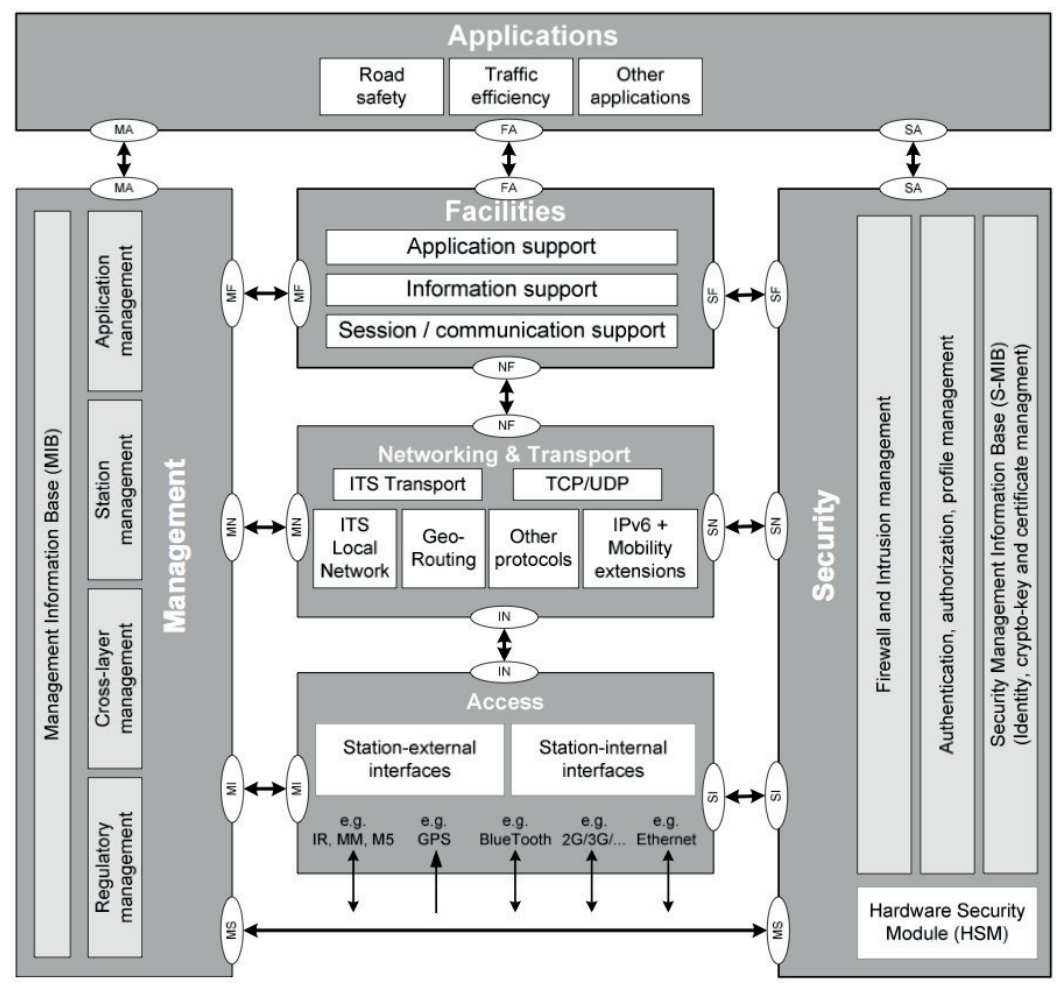

Figure 7.1: ETSI architecture of an OBU [32] 


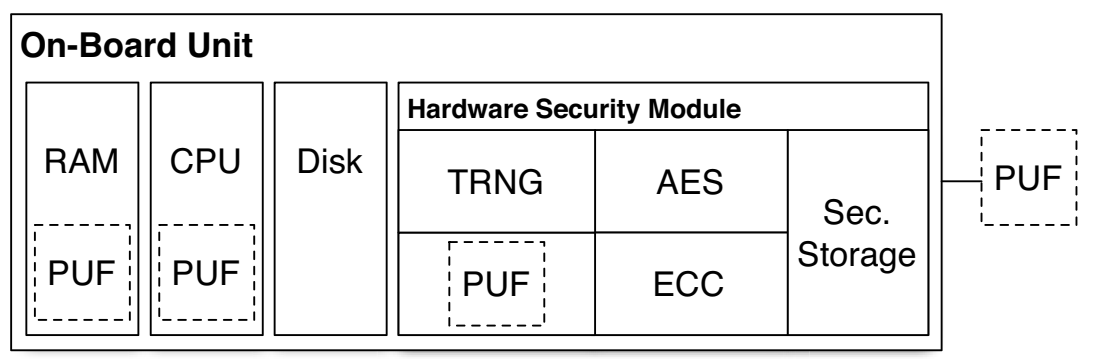

Figure 7.2: Simplified hardware architecture of an OBU

(TRNG), cryptographic primitives (AES, ECC), secure storage, and a PUF. However, one should notice that the PUF could be outside of the HSM (represented in dashed line in Figure 7.2).

Indeed, the PUF could be fully integrated in the CPU, GPU or RAM [84], but also attached to the OBU as an external device. We consider the PUF as an external device as we compare against classic secure storage solutions (e.g. smart card, secure token), which are mostly externally attached to the OBU. A consequence of being outside the HSM is the lack of a secure computation environment. An attacker (described in Section 7.2.3) could then access to the memory to steal key material. However, this drawback is limited by the restricted lifetime of the pseudonyms (i.e. certificate). We further discuss the issue of secure computation in Section 7.5.2.

\subsubsection{Attacker Model}

With respect to secure storage we consider attackers who want to access the content that is placed in the secure storage container. In our context, the aim of the attacker is to copy the private key material used as pseudonym of a vehicle. We differentiate between two attacker goals: An attacker might try to get access to the private keys for the currently used pseudonym or the attacker might aim to access all private keys for all pseudonyms provisioned in the OBU.

An attack against the OBU can be performed by injecting a payload into the system, which would trigger malicious actions. Since the OBU does not provide a user interface, such a payload needs to be injected into the system remotely. OBUs offer a number of opportunities to an attacker to input data into the system remotely. Most notably the networking and communication applications in the OBU are processing data from external sources, which might be controlled by an attacker. Exploitation of security holes in these applications can lead to different levels of access to the contents of the OBU:

1. Access to filesystem data 
2. Access to application memory

3. Access to hardware devices and code execution

We consider attackers with an escalating set of capabilities to evaluate the level of protection offered by the different proposed techniques. Access to filesystem data serves as a baseline scenario to illustrate that the basic secure storage mechanisms work. An attacker should never be able to access key material based on filesystem access. The second level of access represents more severe information disclosure attacks. In a scenario without secure computation, an attacker can extract key material that is currently in use. A third type of attacker has the ability to execute arbitrary code on the OBU, and thus, is able to arbitrarily interact with any device attached to the OBU. For an external device, such an attacker is indistinguishable from a regular host application. Nevertheless, we consider this type of attacker as the most powerful type of attacker, because she has full control of the OBU.

Hardware attacks against the secure storage are not considered in this context. The intrinsic tamper-resistance of PUFs is assumed to protect against this kind of attacker. We assume equally that the tamper-proof enclosure of classic secure storage solutions is effective.

\subsection{CLASSIC SECURE STORAGE}

In this section we propose ways to implement efficient secure storage of large numbers of private keys for use in secure pseudonymous communication. We differentiate between regular storage and secure storage requirements for keys and related support data. The proposed solutions have different space requirements to store and protect these data, which is our main metric to compare the efficiency of the proposed methods. As a baseline, we assume the availability of classic external secure storage, for example in the form of a physical smart card or as part of a dedicated secure storage token on a USB device. Our goal is to minimize the usage of this resource or eliminate the use of this resource entirely.

\section{7·3.1 Individual key storage}

The canonical way to handle secure storage is to assume the presence of a dedicated device, which is isolated from the host. The security attributes of this solution are derived from the fact that the memory on this type of device is only accessible through a well-defined security API.

No other way should exist to access the data, neither in software nor in hardware. The protection against hardware access is usually achieved through protective tamper-proof enclosures or self-destructive 
coating. The details of the hardware and the communication protocol as well as options to perform secure computation on the device are out of the scope of our work.

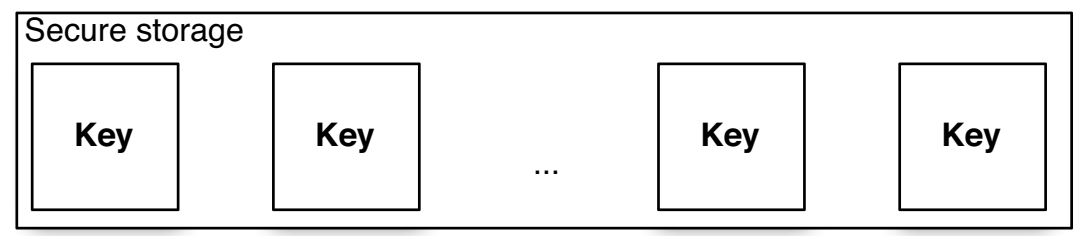

Figure 7.3: All keys in secure storage

Figure 7.3 illustrates the fact that all $n$ keys need to be stored in the secure external device. The limiting factor of this solution is the raw amount of data that needs to be stored in this scheme. As introduced in Section 3.2, it is expected that secure pseudonymous communication in vehicular networks will require storage of large numbers of preallocated private keys. The key management and the amount of secured data storage increase the cost of such a solution as the number of pseudonyms grows.

\subsubsection{Encrypted storage}

Storing private keys in encrypted form in regular storage, e.g. in a file or database, is a common solution found in password management software for consumers (see Section 7.1.3). This kind of solution is usually tied to a master password and a password-based key derivation function to decrypt the data structure. For non-interactive use, we can adapt this solution to use a master key stored inside a secure storage device to encrypt and decrypt the private keys as needed. Figure $7 \cdot 4$ illustrates this method. Using a master key with sufficient entropy in a secure data store allows us to avoid key stretching techniques [75] that are typically employed in password based key derivation functions like PBKDF2 [70], bcrypt [110], or scrypt [103].

The advantage of this method compared to a classic secure storage solution (Section 7.3.1) is that only one master secret is required to be stored securely. This master secret will subsequently unlock any number of additional private keys, which can be stored in encrypted form in regular unsecured memory. Conversely, the disadvantage is, that now an attacker only requires this master key and the encrypted-but not securely stored-data structure of private keys. This compromises not just one private key, but all private keys stored in this data structure. 


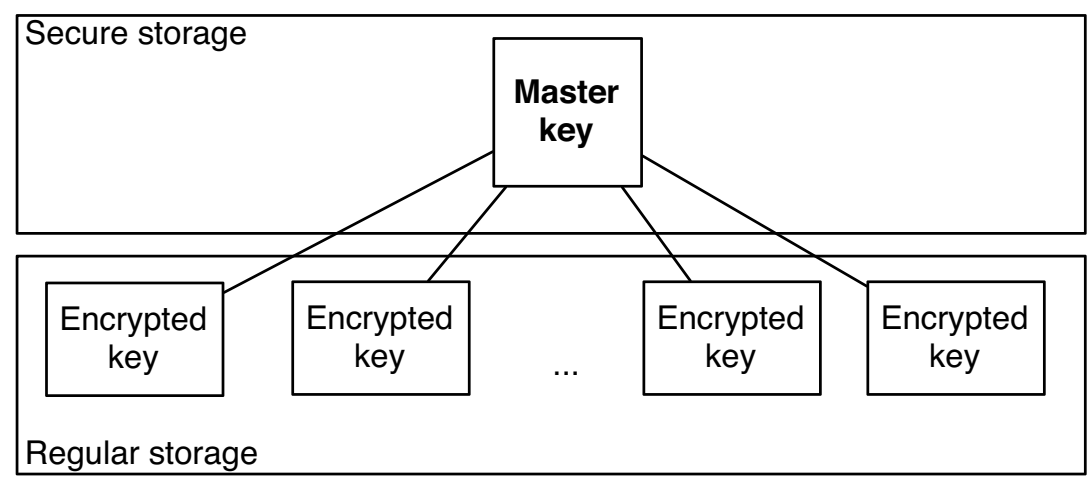

Figure 7.4: Keys retrieved from encrypted file in regular storage using a securely stored master key

\subsubsection{Key derivation}

Taking the concept of using a master secret even further, we use a key derivation function to derive secret keys from the master secret. A practical implementation of this idea uses a keyed pseudo-random function to derive a sequence of bits from a single master key (or seed). These bits can be used as a secret key for symmetric cryptography, but also as a deterministic source of random bits in the generation process of an asymmetric ECDSA key pair [46]. Figure 7.5 illustrates this abstract process. Well known constructions of such key derivation functions include KDF2 [42, 64, 66], HKDF [22, 82, 83], and the set of deterministic random bit generators (without reseeding) recommended by NIST [3].

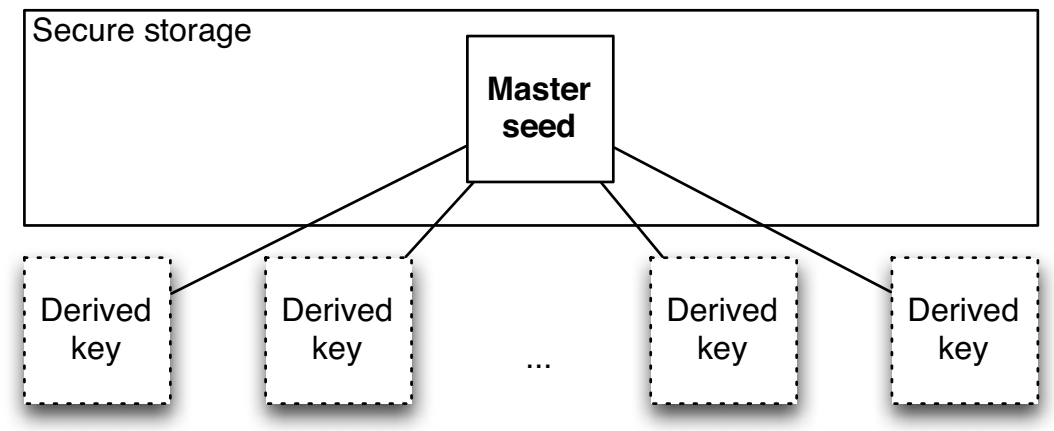

Figure 7.5: Keys regenerated through a key derivation function using a securely stored master key 
An additional advantage of using key derivation functions is the reduction of the communication overhead. Indeed, if the CA generates and stores the master seed for the vehicles, it is no longer necessary to submit the key pair through a secure communication channel to the vehicle. It is enough to transfer only the certificates, which need to include context information, to allow the vehicle to derive the matching key pair independently. This information do not even require protection, enabling the use of unauthenticated broadcast channels or public certificate servers for the delivery of new pseudonym certificates.

\section{$7 \cdot 4$ PUF-BASED SECURE STORAGE}

In this section we propose secure key storage solutions that do not rely on any classic external secure storage, but instead use Physical Unclonable Functions (PUFs) to achieve the desired security. As introduced in Section 7.2.1, we consider two types of PUFs: Strong PUFs and Weak PUFs.

\subsubsection{Strong PUF-based secure storage}

Ongoing research on applications of PUFs for key generation and regeneration is focusing on the fuzzy extraction algorithm. From an application perspective in the vehicular communication context, we need to securely store large numbers of secret keys. Our proposal, which is summarized in Figure 7.6, requires the use of a Strong PUF [117] that fulfills the following requirements:

1. It must be impossible to physically clone the PUF.

2. A complete determination/measurement of all challenge-response pairs (CRPs) within a limited time frame (such as several days or even weeks) must be impossible.

3. It must be practically impossible to numerically predict the response to a randomly selected challenge, even if many other CRPs are known.

These requirements were setup by Rührmair et al. with scenarios in mind that require a large number of interactive challenge-response cycles, e.g., for remote authentication. Attackers could, e.g., send specific challenges to the PUF, record the responses, and then try to perform a so called "model building attack" [117]. For our usage of PUFs for pseudonym storage, an attacker will not be able to directly query the PUF and see the responses. Only the CA is supposed to be able to communicate with the OBU, and PUF responses will only be used to derive key pairs from it. This effectively removes the unconditional 
need for requirement two, although for cost effectiveness of this solution it is still desirable to demand a large space of challenge-response pairs (CRPs). In Section 7.4.2, we propose an alternative solution that can tolerate the availability of only small amount of CRPs per PUF (Weak PUF).

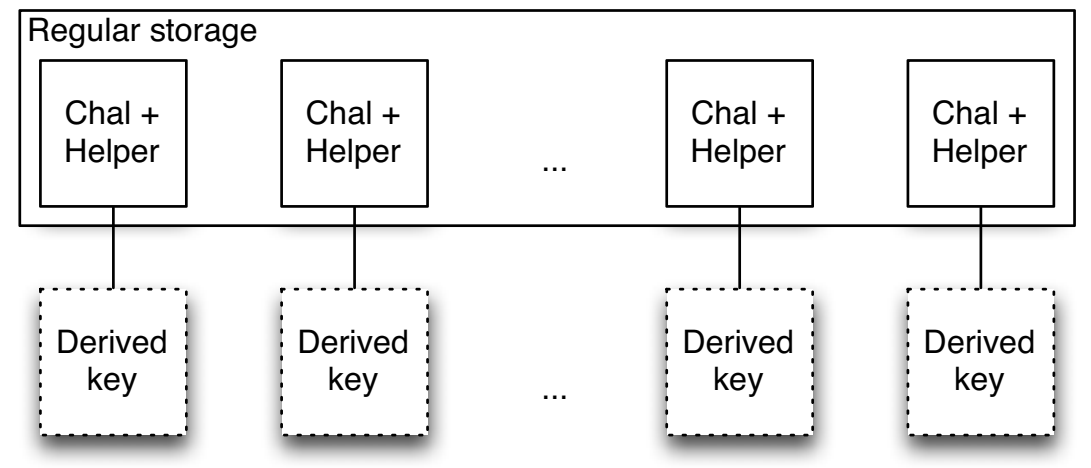

Figure 7.6: Keys reconstructed securely from a strong PUF using regularly stored challenges and helper data

The idea that we pursue in this proposal is to derive key material from PUF responses. The use of a Strong PUF implies that we have a large space of challenge-response pairs, which enables us to derive large numbers of keys. As in the solution based on KDF, we use deterministic random bits as a source of entropy in the key generation process of asymmetric ECDSA key pairs [46].

The amount of input data required to generate a stable amount of responses is highly dependent on the attributes of a concrete PUF construction. In general, we require a set of chosen challenges and a set of helper data, which is generated by the fuzzy extractor during the initial key generation process. Depending on the type of PUF construction, a total amount of $n$ challenges $c_{n}$ of $x$ bits length is required to generate $m$ bits of output. These $m$ bits of output then need to be stabilized using a fuzzy extractor (see Section 7.2.1). In the initial key generation process the fuzzy extractor will generate helper data. In subsequent calls to the PUF, this helper data is used by the fuzzy extractor to reconstruct the same stable response. In both cases, the fuzzy extractor will consume a percentage of the data for entropy compression and error correction. The factor of the data reduction $r$ as well as the length $y$ of the helper data $W$ depends on the type and configuration of the fuzzy extractor. The configuration needs to be calibrated based on the expected error probability and entropy quality of a given PUF construction. 


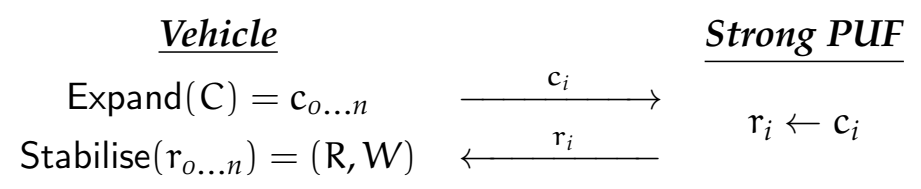

Figure 7.7: An initial challenge (C) gets expanded into $n$ challenges $\left(c_{i}\right)$, which generate responses $\left(r_{i}\right)$ in the PUF. The vehicle combines these into a final response $(R)$ and helper data (W).

For an overall amount of stable response bits $z$, we can calculate the number of required challenges as $n=\frac{z}{\mathrm{~m} \cdot r}$. To enable reconstruction of stable responses, we would need to store the $n$ challenges of size $x$ and the helper data $W$ of length $y$. Regarding the choice of challenges, we note that to ensure the independence of output bits we need to avoid repetitions of challenges. A simple increment function allows us to easily expand multiple challenges from an initial challenge, while avoiding collisions and covering the whole space of possible challenges optimally. This makes it possible to only store the starting challenge and derive all following challenges.

Thus, to enable reconstruction of fixed size stable responses, we need to store only the starting challenge of size $x$ and the helper data $W$. Once all possible challenges are exhausted, the PUF should not be reused ${ }^{3}$.

Requirement three of the Strong PUF definition, as well as the attributes of the fuzzy extractor, must ensure that even just a one bit difference between challenges guarantees a fully independent response.

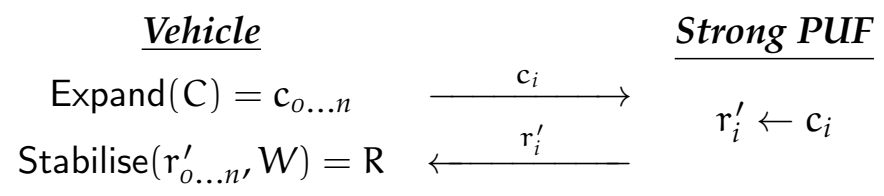

Figure 7.8: Regeneration of responses is analogous to the initial provisioning, except the previously generated helper data (W) is now utilized by the Stabilise() function to stabilize the response.

The details of the ECDSA key pair generation process are specified in [46]. For example, a fixed amount of 320 random bits are required to deterministically build a key pair of 256 bits. Thus, we assume a need of $z=320$ bits of stable entropy from the PUF to be able to generate a 256 bit ECDSA key pair.

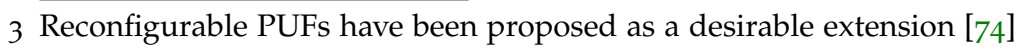


Once the vehicle has constructed its key pair as outlined above, it can then build and submit a certificate signing request (for the public key) to the CA through a authenticated and integrity protected channel to trigger the certification process. The CA subsequently returns a signed certificate, which completes the provisioning process of a new pseudonym.

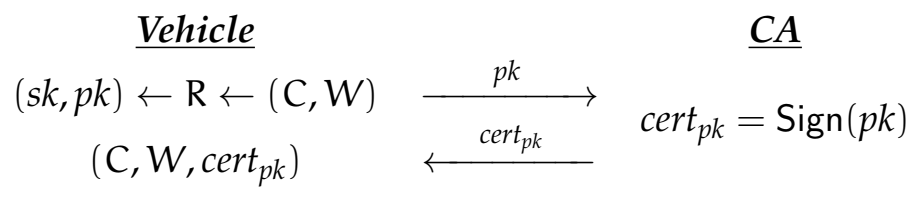

Figure 7.9: The vehicle generates an asymmetric key pair from a challenge $C$ and helper data $W$. The CA creates a certificate for the public key $p k$, which is stored in the vehicle with $C$ and W.

This method of secure key generation and key reconstruction from PUFs completely avoids any need for classic secure storage. The starting challenge and helper data can be stored in regular storage space. The security of the key material is fully guaranteed by the need to have access to the related PUF device with its intrinsic tamper resistant attributes.

\subsubsection{Weak PUF-based key derivation}

A Weak PUF deviates from the definition of Strong PUF by allowing just one fixed CRP per PUF. It can be considered as a PUF that has a fixed built-in challenge and whenever queried provides the same response. This leads to the violation of requirements 2 and 3 of the Strong PUF definition as described above. Nevertheless, even if the Weak PUF has a capacity of one single CRP, this CPR will have a useful amount of entropy. Assuming that the size of the response provides sufficient entropy for a master secret as described in Section 7.3.3, we can apply the same technique here.

An illustration of the two stage key derivation process is shown in Figure 7.10. First, a master key is derived from the response of a Weak PUF. Then, this master key is used as a seed to derive the key material for multiple pseudonyms. For instance, the PUF response could be used as the "input keying material" for the Extract function of HKDF [83]. 


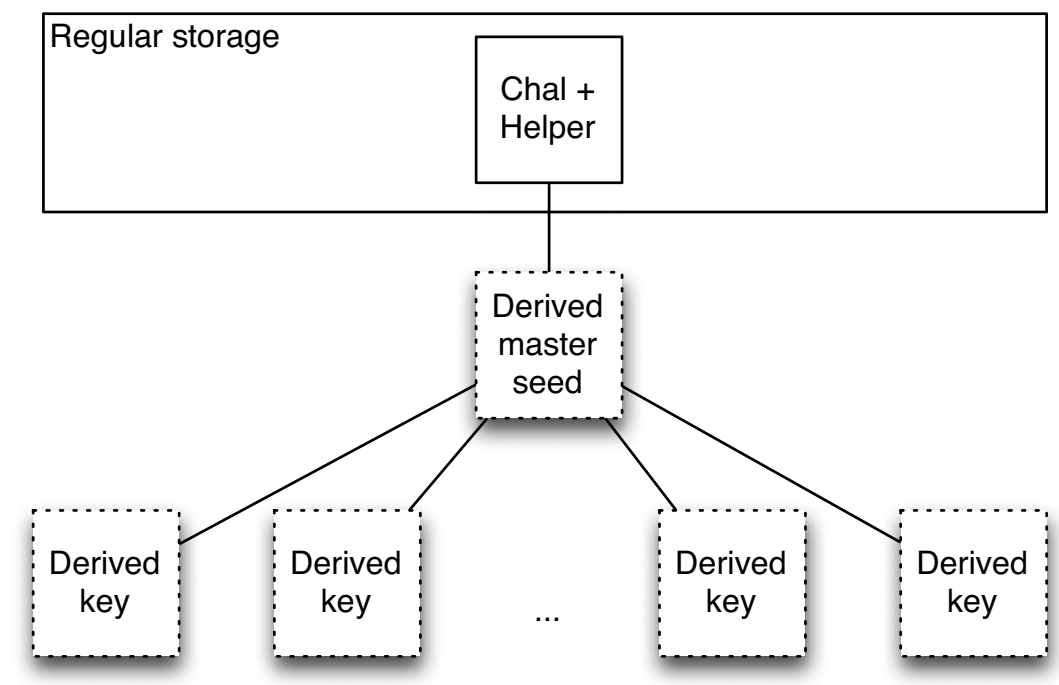

Figure 7.10: A master key gets reconstructed securely from a weak PUF using regularly stored challenges and helper data and is then used to regenerate derived keys. 


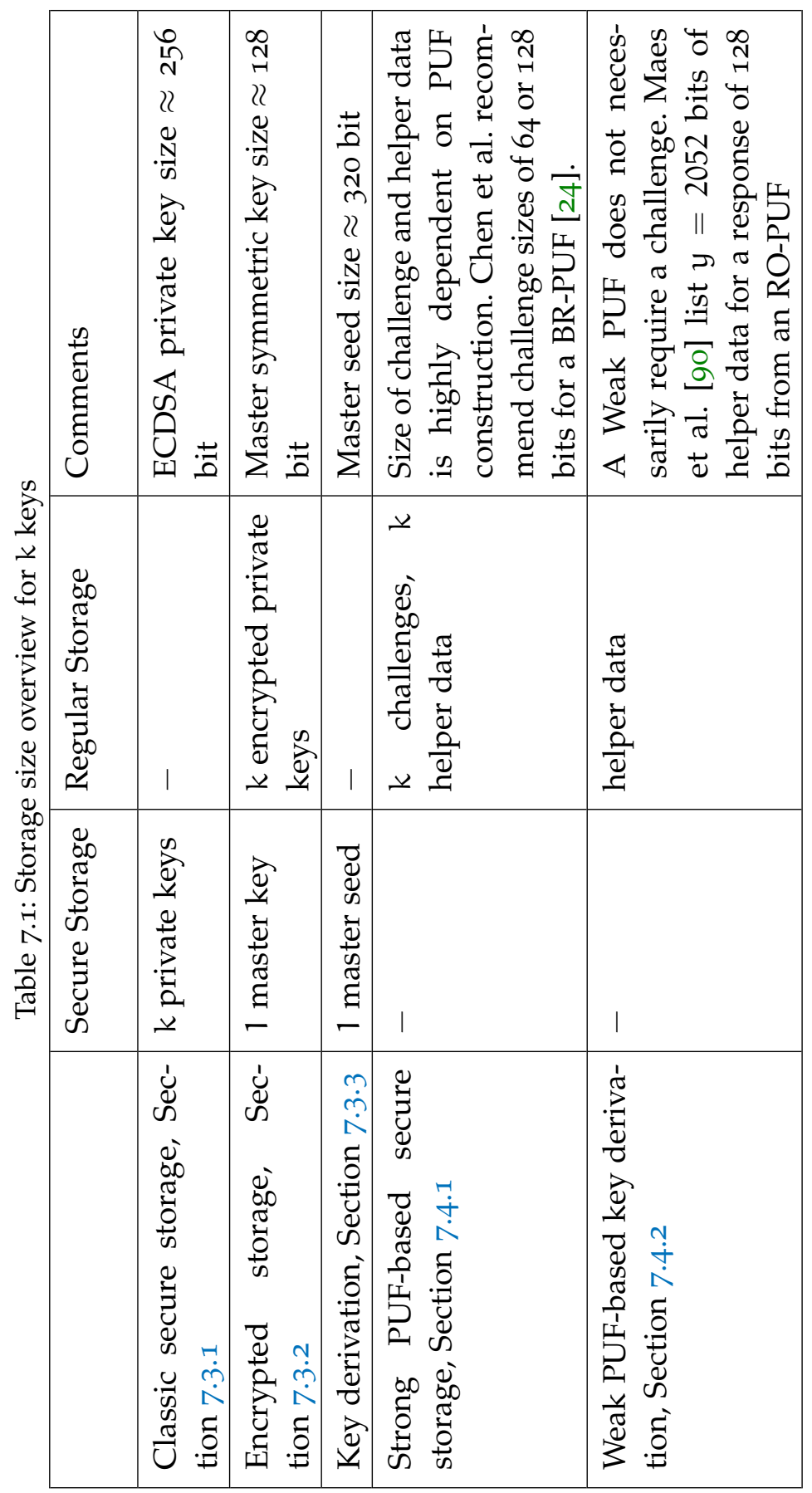




\subsection{Discussion}

The presented solutions for the secure storage of key material for pseudonyms employ Key Derivation Functions (KDF) and Physical Unclonable Functions (PUF) to achieve multiple levels of efficiency improvements. The two major aspects for the evaluation of the solutions are the storage requirements and the security properties with respect to attackers with different capabilities.

In Table 7.1 we summarize the storage requirements of the proposed solutions for the secure storage of $k$ keys. Based on the assumption of storing $k=105120$ keys, the baseline classic secure storage scenario would require approximately 3.2 Mbytes of secure storage space. An encrypted data structure, as described in Section 7.3.2, would allow to drastically reduce the amount of secure storage. In this scenario, the full 3.2 Mbytes of encrypted key material still has to be stored, but it can be stored in regular memory.

The use of a key derivation function removes this requirement of regular storage by relying purely on a master seed value, which is used to generate key material on-the-fly.

The solution based on the application of a Strong PUF does not require any classic secure storage device at all. Instead, it is possible to rely solely on the intrinsic security of the PUF construction. However, the amount of regular storage space required to regenerate keys is larger than the raw amount of private keys. This is due to the need for helper data, which is required to stabilize the readings of responses from the noisy hardware constructions of PUFs. The exact amount of required helper data and the size of challenges are highly dependent on the attributes of a given PUF and also on algorithmic choices of the fuzzy extractor.

Finally, we see that a combination of PUF and KDF techniques even allows us to present a solution that technically does not require any secure or regular storage at all. The Weak PUF using just one challengeresponse pair returns its response without any explicit challenge, simply by virtue of being powered on.

The second criteria to compare the proposed solutions is the resilience against attackers with different levels of capabilities (see Section 7.2.3). Table 7.2 gives an overview of the security properties. We see that all solutions guarantee the basic requirement of denying any access to the key material to an attacker who has access to the regular unsecured filesystem. 


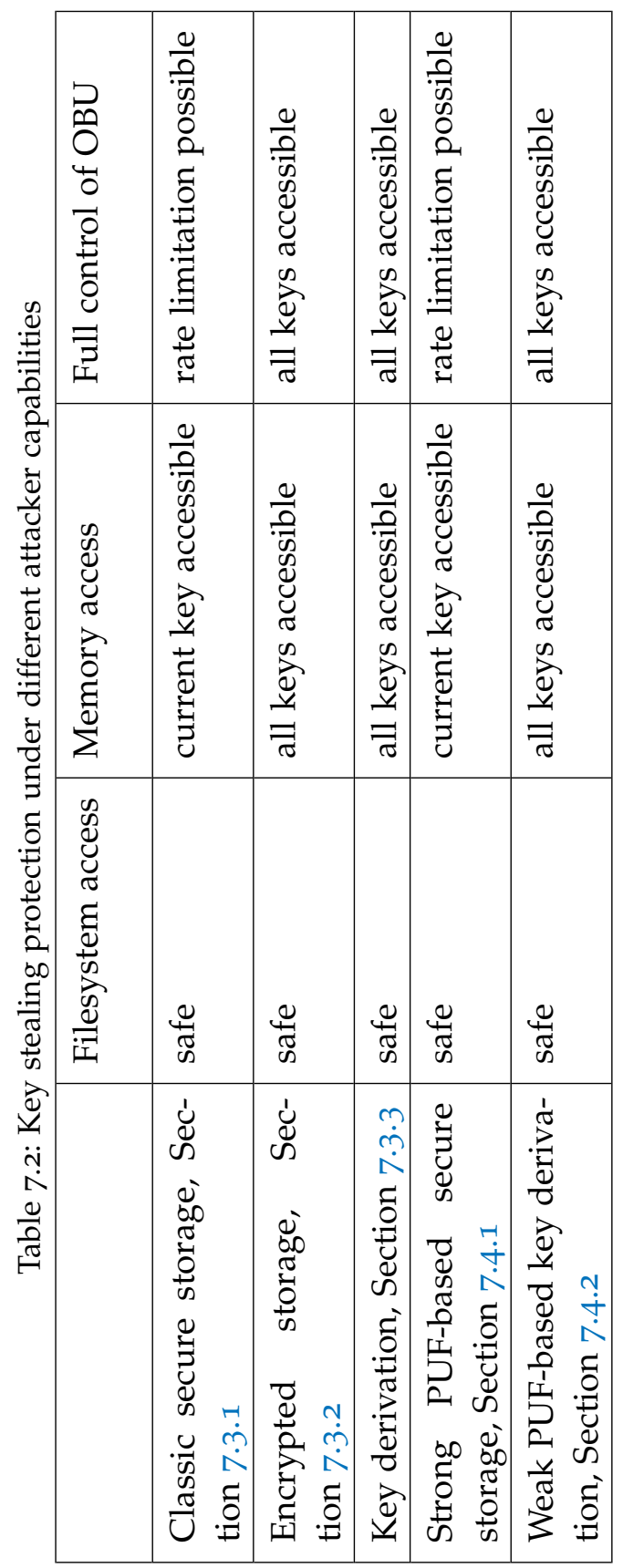


As described in Section 7.2.3, the next level of attacker capability grants the attacker read-only access to arbitrary regions of OBU memory. The attacker might have found an exploitable bug in the software and injects malicious code to extract valuable data. We see weaknesses in three of the proposed solutions, due to the fact that these rely on a single piece of master secret to derive key material. This master secret (a master key or a master seed) has to be extracted from a classic secure storage device or from a PUF and is identical for all keys that are derived by the system. An attacker with the capability to observe the address space of the application can potentially copy this master secret during the derivation process of any key. The attacker can then derive all possible keys based on this master secret. Only the pure classic secure storage solution and the Strong PUF based solution are not affected by this issue, because these solutions derive all keys independently.

The final model grants the attacker full control over the host, which implies code execution privileges and direct access to the device. Generally, there is no way to protect the information against access by such a powerful attacker, because the storage device cannot see a difference between normal usage and usage by such a powerful attacker. One additional option to offer a mitigation against malicious use could be a rate limitation mechanism, which limits the number of requests over time. For the use case of pseudonymous communication in vehicular communication it could be sufficient to only return one key per minute. Such a feature represents a viable security benefit, because the attacker can effectively only make use of the attacked device while it is online. The classic secure storage solution, as well as the Strong PUF-based solution, could reasonably offer such a feature.

\subsubsection{Limitations of KDFs and PUFs}

In the previous section, the comparison of the security properties listed in Table 7.2 shows that the existence of a single master secret, as it is the case in the KDF-based solutions, represents a disadvantage under certain attacker models. Another issue to consider is the limitation of the number of keys to derive from one single master key. It is advisable to rekey the system after a certain amount of keys was derived. The rekeying interval depends on the construction of the underlying algorithm used in the KDF. This also highlights the abstract disadvantage of having to rely on additional cryptographic algorithms compared to the solutions that access keys without intermediary derivation steps. More exposure to cryptographically strong algorithms naturally implies more risk of being affected by a discovery of a weakness in such algorithms. 
Similar concerns are valid for PUFs, where the fuzzy extraction process is comparable to a key derivation process. The complexity of these processes might enlarge the exposure to bugs and weaknesses. Moreover, there are fundamental capacity limits (i.e. challenge-response pair space) that might impede practical deployments. Since PUFs are intrinsically bound to hardware, it might be impossible to reuse (rekey) a PUF after the capacity limit is reached. This is particularly problematic for Weak PUFs with only one or a very limited number of challengeresponse pairs. Controlled PUFs and Reconfigurable PUFs [74] have been proposed as solutions for this problem, but the feasibility of such constructions is difficult to evaluate. A controlled PUF would be particularly desirable in the context of secure storage for the possibility to effectively implement rate limitation in hardware.

While it is not an issue for pseudonyms storage in vehicular network, we acknowledge that the speed of accessing a PUF can be a limiting factor. The secure key reconstruction from PUFs incurs a considerable amount of computational overhead for the fuzzy extraction of responses. According to [90] the execution time is in the order of magnitude of several milliseconds for an RO-PUF design. Additionally, the challenge $C$ and helper data $W$, which need to be stored for the regeneration of a stable response, are significantly larger than the plain private key. While we propose an expansion function to avoid storing all challenges $c_{n}$, the helper data can easily add up to several kilobytes in order to generate stable response data [90].

Another limitation of using PUFs for key generation and key storage is that PUFs are effectively read-only devices. Therefore, it is necessary for vehicles to create key pairs locally, using the response of a PUF challenge as a controlled source of entropy.

We summarize the limitations of PUFs as follows:

1. Read-only data store

2. Limited capacity

3. Readout time

4. Faith in fuzzy extractor algorithms

5. Need to store helper data

These limitations pose restrictions on the realm of possible applications for PUF-based secure storage. PUF-based solutions are consequently not suitable as a direct universal replacement for all applications of classic secure storage. Nevertheless, when these limitations are met, the use of PUF-based solutions is a secure and efficient option to replace classic secure storage.

\subsubsection{PUF integrated within an HSM}

As shown in Figure 7.2, the PUF could be inside an HSM. Then, our schemes would benefit from this secure computation environment. 
Indeed, an HSM commonly provides secure memory, secure storage, and secure cryptographic primitives. This solution ensures that the key is generated and used at the same place, and never leaves the HSM. In this case, one can notice that integrating the PUF inside the HSM will prevent all the key stealing attacks listed in Table 7.2.

However, an attacker with full access could still use the HSM to perform malicious actions such as signing forged message. Moreover, the PUF limitations still hold even within an HSM. For instance, the limited capacity of the challenge space triggers the question about what would happen when a CRP space is depleted. As no Reconfigurable PUF exists yet, replacing the HSM would incur a considerable cost.

Finally, we conclude that if secure computation is assumed, then the cost-benefit advantage of PUF is questionable. We note that the encrypted storage model (Section 7.3.2) would not suffer from any limitation of the PUF-based solutions while offering a better tradeoff between secure storage and regular storage. According to Table 7.1, encrypted storage needs I private key and k cipher texts, while PUF-based approaches require no private key but $k$ challenges and $k$ helper data. One should notice that, in terms of size, the cipher text is significantly smaller than the set of challenges and helper data.

\subsection{CONCLUSION}

We propose to use encryption and key derivation functions to reduce the need for secure storage. Our comparison shows that these techniques are effective at reducing the requirements for secure storage at the cost of reduced protection against attackers with access to host memory. We alternatively propose to use Physical Unclonable Functions (PUFs) to eliminate the need for classic secure storage entirely. Our analysis shows that PUFs can effectively replace classic secure storage if an application can operate under the limitations of a given PUF. The use-case of secure pseudonymous communication in vehicular networks is generally compatible with these limitations.

The attractiveness of PUF-based solutions is a result of potential cost savings compared to more expensive secure storage. PUFs are envisioned to be cheap enough for inclusion in mass produced RFID tags or might already exist in common hardware. This represents a considerable cost-benefit advantage. Once the availability of hardware implementations increases, we expect PUF-based solutions to see widespread use in practical applications.

In this work we require two properties of PUFs that allow us to implement optimizations and make assumptions about the security of the overall system:

1. A one bit difference between two challenges is enough to guarantee completely independent responses. Knowledge of related 
(not randomly selected) challenges does not affect the unpredictability of responses.

2. Knowledge of helper data does not reveal any information about the expected response from a PUF.

These attributes are implied by the Strong PUF requirement 2 and by the fuzzy extraction algorithm goals. However, usually no explicitly guarantees of these attributes are given in the design documents of concrete PUF constructions.

Applications of secure storage in vehicular OBUs often involve full Hardware Security Modules (HSM) to provide secure computation in addition to secure storage. Rate limitation and limited lifetime of certificates allow operation without secure computation. It remains an open question, if a PUF-based secure storage solution can be augmented to offer secure computation, while retaining a cost-benefit advantage over classic implementations in an HSMs. 
ACCELERATED CRYPTOGRAPHY FOR SECURE VEHICULAR COMMUNICATION

Offloading cryptographic operations to dedicated hardware can be an enhancement for operational confidentiality, because it allows the key material to be inaccessible to a host machine. However, another major benefit of exclusively dedicated hardware is predictable performance. In contrast to shared resources, which need to be managed with appropriate allocation and scheduling algorithms, this allows confidence in hard availability guarantees even under high load. In safety relevant cases it can be mandatory to provide dedicated hardware for acceleration of cryptographic operations to provision enough computational resources. The performance requirements for computational processing of cryptographic security services have been defined in Part II.

Here we investigate the relevance of cryptographic acceleration as a provisioning option for the unconditional availability of cryptographic security services. For this purpose, a set of empirical benchmark measurements is collected on generic on-board units (OBUs). These OBUs come from a selection of devices that have been used within the European research project PRESERVE (Preparing Secure Vehicle-to-X Communication Systems) and were used in European vehicular communication trials. The measurements provide a reference for the software performance of raw cryptographic algorithms as well as the performance of complete security processing services.

\subsection{A REPRESENTATIVE VEHICULAR SECURITY SUBSYSTEM}

Vehicular communication is a technology that has not reached the deployment stage yet. However, a number of field operational trials have been performed and dedicated research projects have investigated attributes of this technology. Among these research projects was the European project PRESERVE, which had the mission to design, implement and test a secure and scalable V2X Security Subsystem for realistic deployment scenarios. We use the $\mathrm{V}_{2} \mathrm{X}$ Security Subsystem Kit (VSSKit) produced by the PRESERVE project as a representative close-to-market implementation of the relevant security components 


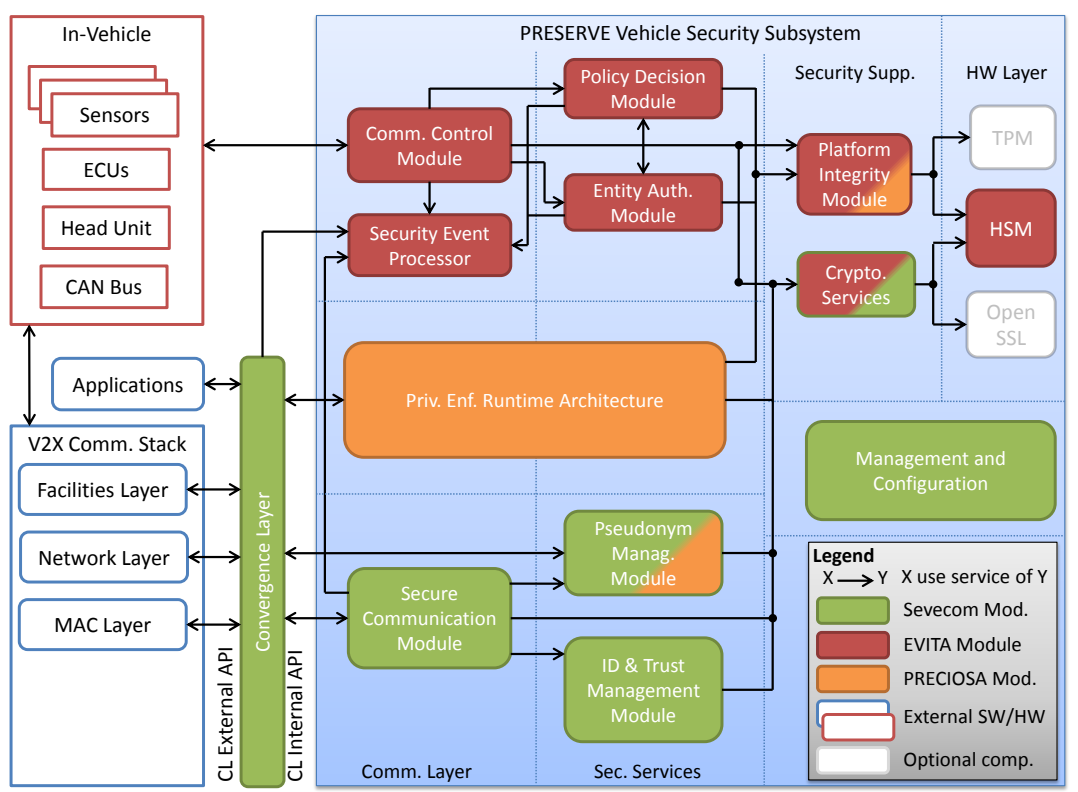

Figure 8.1: Architecture of the PRESERVE V2X Security Subsystem [13]

in a realistic vehicular communication architecture. Figure 8.1 shows an architectural overview of the VSSKit, which we use as a foundation for realistic performance evaluations of cryptographic services for $\mathrm{V}_{2} \mathrm{X}$ communication.

To evaluate the performance characteristics of the PRESERVE VSSKit we collected benchmarks of the basic sign and verify operations, which provide the foundation for the security of vehicular communication. These operations are sometimes referred to as encap and decap, to highlight that the encapsulation and decapsulation of messages can include more work than the application of raw cryptographic primitives. The measurements are taken at the external API of the PRESERVE architecture in order to collect realistic overall performance data. In Figure 8.1 this location is labeled as the Convergence Layer.

An overview of the control flow for incoming message verification in the PRESERVE VSSKit is shown in Figure 8.2. The execution flow involves the Convergence Layer, the Secure Communication Module, The ID \& Trust Management Module, Cryptographic Services and one cryptographic backend service, such as a hardware security module or a software implementation or cryptographic primitives like OpenSSL. The involvement of a Security Event Processor or the PRECIOSA Privacy Enforcement Runtime Architecture was optional and not used during the measurements. The Pseudonym Management Module only 


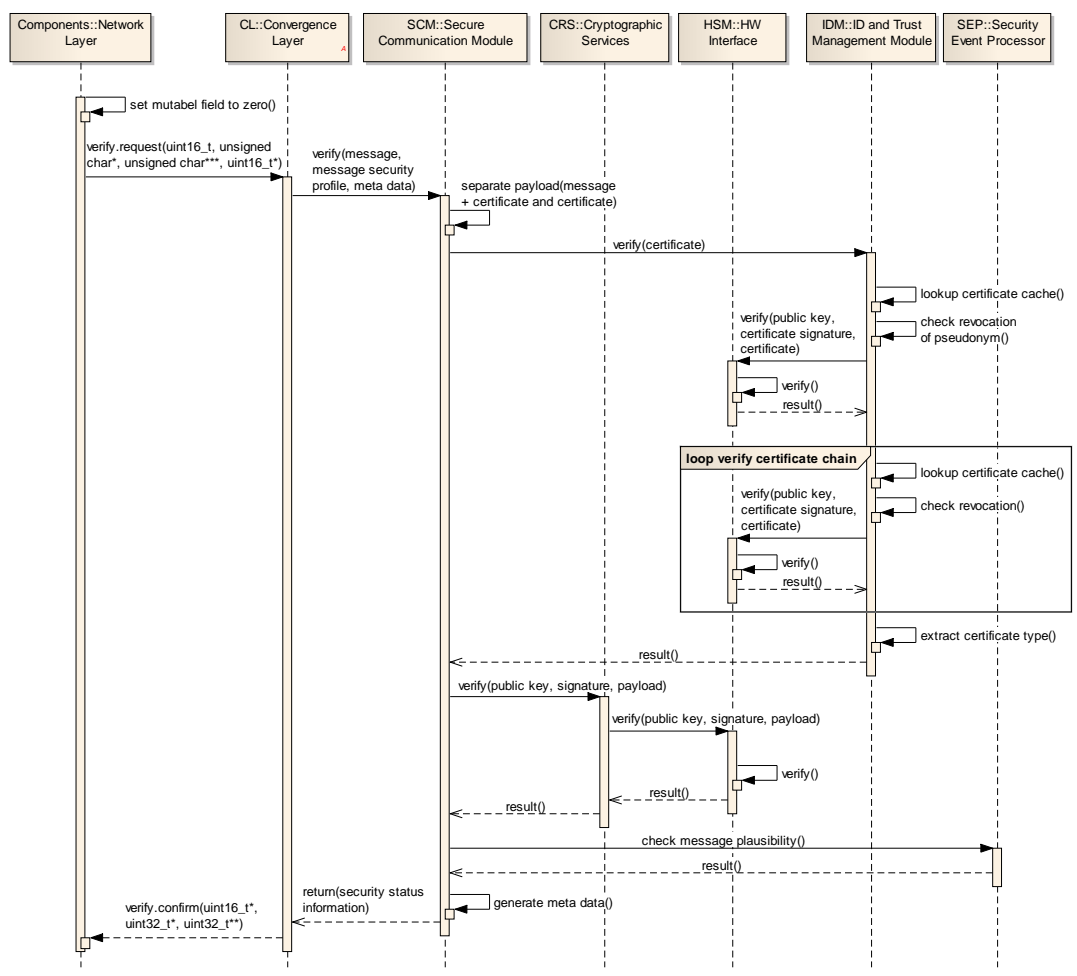

Figure 8.2: Control flow of incoming message verification in the PRESERVE VSSKit [13]

becomes active for pseudonym changes, which is triggered independently from the execution flow of message verification. Equivalent considerations apply to the case of message signature creation.

To derive the overhead introduced by trust management tasks, such as parsing of certificates and validity checks of certificate attributes, we also present benchmarks of raw cryptographic primitives of the cryptographic backends. The PRESERVE VSSKit supports multiple cryptographic backend through a flexible Cryptographic Services module. Measurements were collected for the following backends:

1. OpenSSL libcrypto (software)

2. VSSKit vi FPGA (hardware)

3. VSSKit v2 ASIC (hardware) 


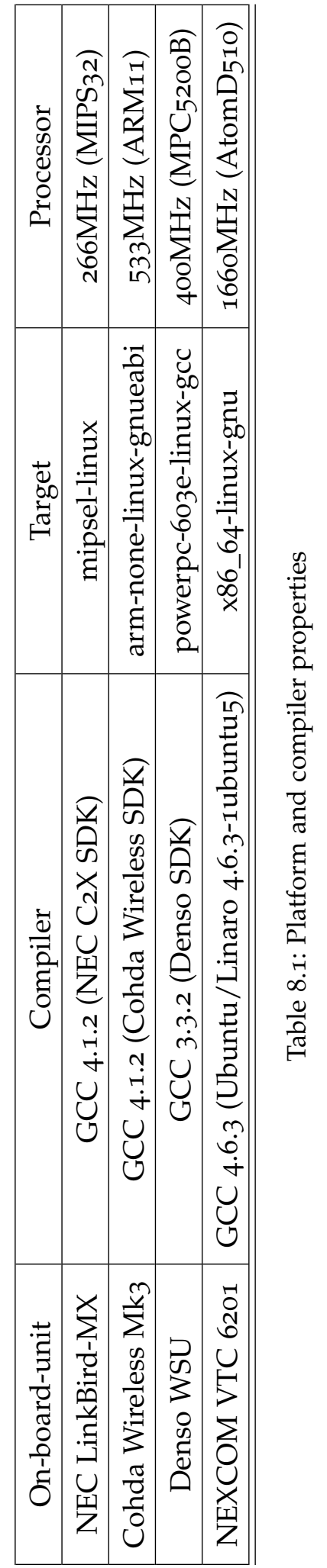




\begin{tabular}{|c|c|c|c|c|}
\hline Setup \# & sign & sign/s & verify & verify/s \\
\hline NEC Linkbird-MX & $28 \mathrm{~ms}$ & 35 & $110 \mathrm{~ms}$ & 9 \\
\hline Cohda Wireless Mk3 & $10 \mathrm{~ms}$ & 100 & $35.5 \mathrm{~ms}$ & 28 \\
\hline Denso WSU & $9 \mathrm{~ms}$ & 111 & $29 \mathrm{~ms}$ & 34 \\
\hline NEXCOM VTC 6201 & $1.7 \mathrm{~ms}$ & 588 & $6 \mathrm{~ms}$ & 166 \\
\hline \hline
\end{tabular}

Table 8.2: Single core sign and verify test results with PRESERVE VSSKit v1.4 using the OpenSSL backend

\subsection{PLATFORMS FOR ON-BOARD UNITS}

The performance of the software backends highly depends on the processor architecture of the host platform and also on the availability of suitable assembly or compiler optimizations for the library. For the measurements performed within our test, we used the compiler toolchain provided by the manufacturer of the host on-board unit. The OBU models that were available for our tests are depicted in Figure 8.3. The key properties of the hardware platforms and the software development kits are shown in Table 8.1:

For the purpose of collecting reference benchmarks of software performance we utilized the OpenSSL backend for all software tests. The libcrypto library was cross-compiled with the listed compiler variants and the optimization options as specified by the OpenSSL default configuration settings. Unless indicated differently, version 1.O.1c of OpenSSL libcrypto was used in all cases. All the platforms were configured to only use one CPU core. The optimization options for the compilation followed the default setting of the OpenSSL library, which resulted in the "-O3" optimization level for code generation on all platforms.

\subsection{LATENCY AND THROUGHPUT}

Table 8.2 shows the key latency (in milliseconds) and throughput (in operations per second) performance values for generating and verifying signatures of secure $\mathrm{V}_{2} \mathrm{X}$ broadcast messages. For the purposes of these tests, it is assumed that the signature algorithm is always ECDSA over the NIST P256 curve. The recorded measurements represent the fastest run of at least 10 executions of a benchmark tool which executes the basic preserve_sign() and preserve_verify() operations. This methodology is applicable because the goal is to derive the speed of the pure operational runtime of these operations without consideration of unrelated interruptions by the operating system or other processes 


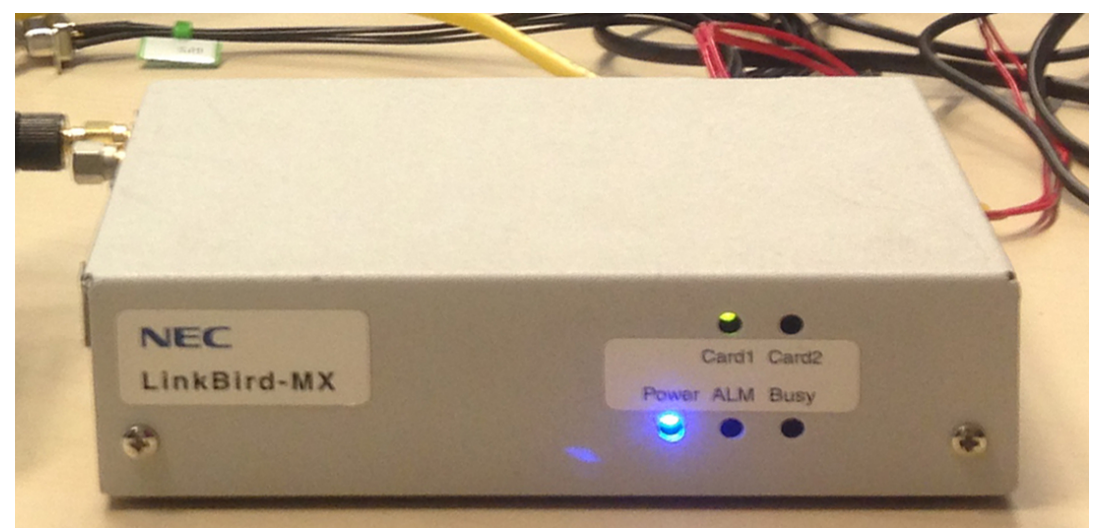

(a) NEC LinkBird-MX OBU

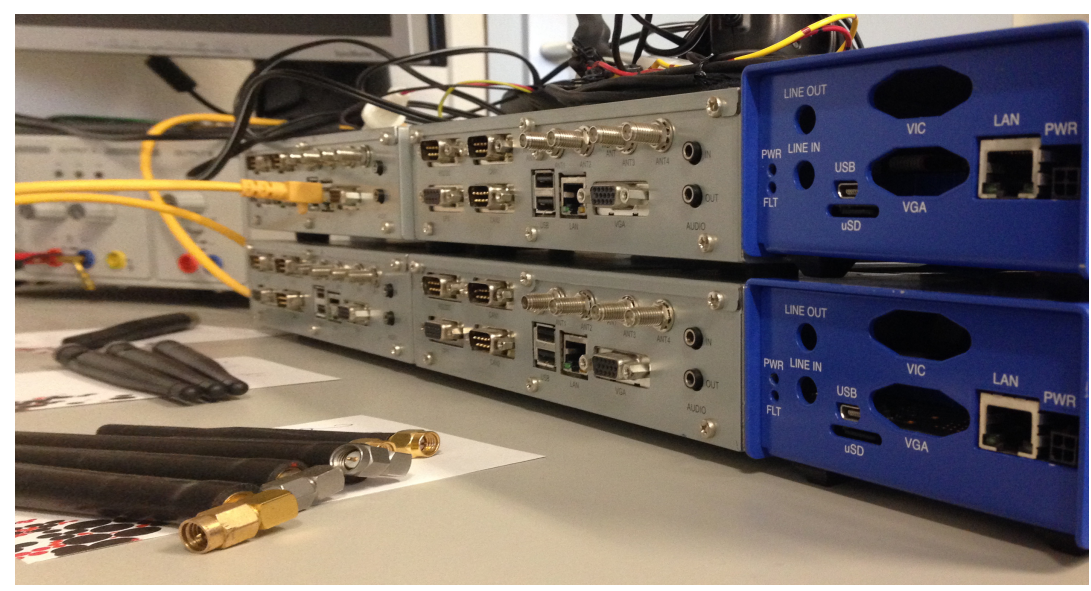

(b) Four Denso Wireless Safety Unit next to two Cohda Wireless Mk3 OBUs

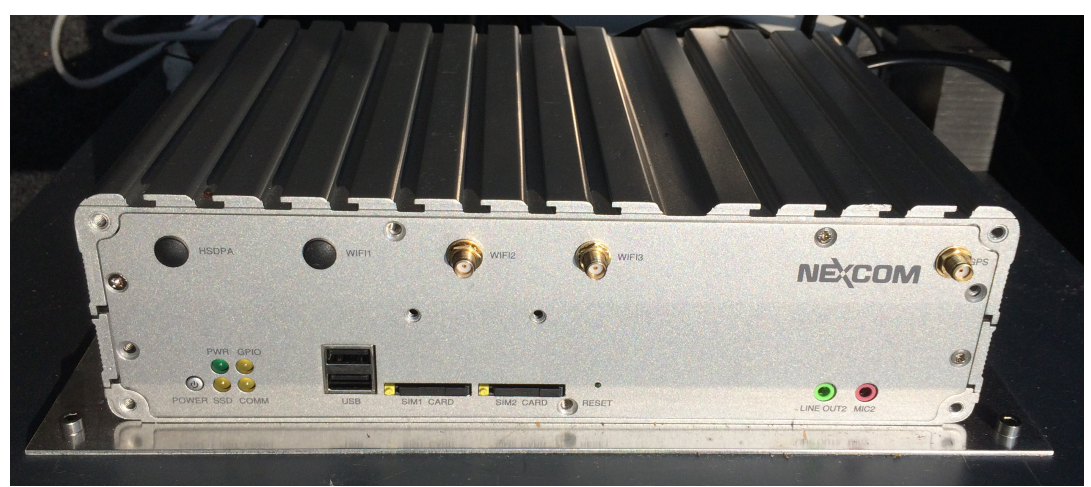

(c) A Nexcom VTC OBU

Figure 8.3: The tested on-board units in various laboratory settings 


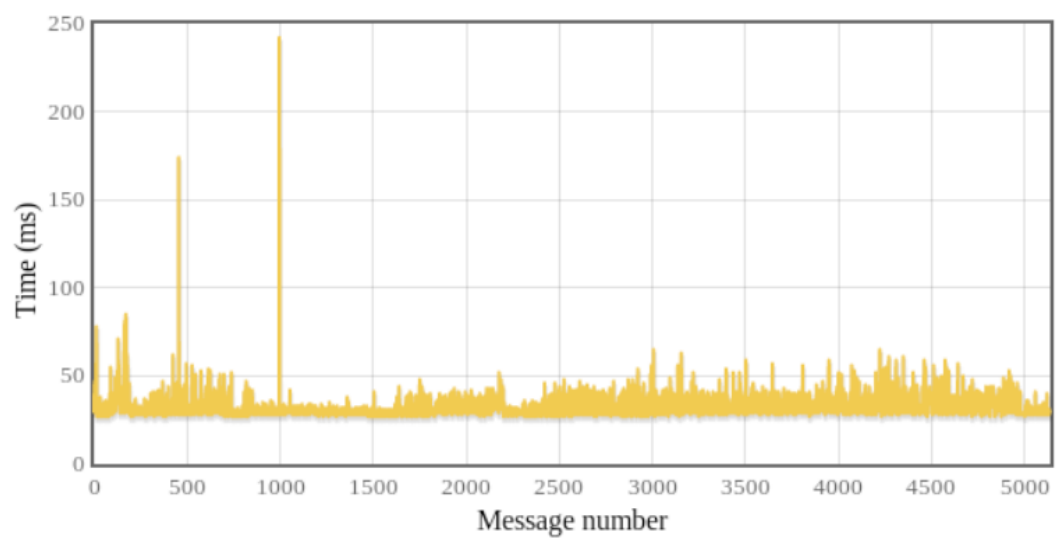

Figure 8.4: Signature verification times for 5000 messages, showing the extent of jitter on a NEXCOM VTC 6201 running $32 \mathrm{bit}$ Ubuntu 10.04 Linux

on the on-board unit. The operating systems did not offer any real time guarantees. Although the host operating systems were kept idle during measurement, it was common to see interruptions and delays, as shown in Figure 8.4.

The results shown in Table 8.2 indicate that all on-board units deliver acceptable performance for signature generation. Based on the requirements discussed in Section 3.4, an on-board unit is expected to generate up to a dozen signatures just for safety related secure broadcast communication. However, all the on-board units fail to deliver acceptable performance levels for signature verification. Previously defined guidelines assert a target performance of up to 1000 signature verifications per second. All tested platforms fall short of this target by several orders of magnitude.

The benchmark results for signature verification with the VSSKit show in Table 8.2 are normalized to show performance for the verification of only the signature of the sending vehicles, which is the final element of the associated certificates trust chain. This is the most common mode of operation, since the signatures of intermediate certificates in the trust chain can be precomputed and/or marked as trusted in the certificate store together with the trusted root certificates. Seeing new unknown intermediate certificates while driving should be a rare occurrence.

In the above benchmark the VSSKit validated the entire trust chain for every call to the preserve_verify() operation. The sample messages used in these measurements verified trust chains with 4 elements, leading to the verification of 4 cryptographic signatures for the verification 
of 1 message. In a realistic deployment, it is expected that trust chains can be shorter and signature verification of some common trust chain elements can be cached. Assuming an unrealistic direct speed up by a factor of 4 , we can extrapolate a latency of $6 \mathrm{~ms}$ for a verification on the fastest evaluated platform, the NEXCOM VTC 6201. This would translate to 166 verifications per seconds, which still falls short of the throughput goal of 1000 verifications per second.

\subsection{PEAK SOFTWARE PERFORMANCE}

All of the above measurements were collected on 32bit systems running one single CPU core. It can be argued that these properties will continue to be typical for embedded systems for a foreseeable future, including the first generation deployment of on-board-units for deployments of vehicular communication technology. For the evaluation of software performance, it is however useful to also consider more powerful processor architectures, which might become available only in the next generation of vehicular on-board units. The NEXCOM VTC 6201 platform can be considered to be a sufficiently powerful platform as to represent the next iteration in the hardware evolution cycle for onboard-units. This system uses an Intel Atom D510 dual core processor with hyper-threading in each core, offering a total of 4 logical CPUs. The system also supports operation in 64bit mode and a set of 128 bit vector instructions, which can accelerate big integer calculations such as those necessary in the relevant variants of elliptic curve arithmetic.

New research results were published in 2013 demonstrating substantial enhancements in the software optimizations for ECDSA over 256-bit prime fields [57]. Accompanying patches have been accepted upstream into OpenSSL and are included in the x86_64 builds of OpenSSL starting at version 1.0.2a. Table 8.3 shows a set of benchmark results, which take all the aforementioned enhancements into consideration. The values show latency and throughput values of raw OpenSSL speed tests as well as values for the PRESERVE VSSKit2 with the same version 1.o.2a of OpenSSL used as a backend.

The signature generation performance in previous test already fulfilled the basic requirements to provide sufficient performance for secure vehicular broadcast communication. Therefore, we focused on analyzing the performance of signature verification results in Table 8.3. The VSSKit2 benchmark setups were configured to not perform full validation of the entire certificate trust chain. This means that only one cryptographic verification is performed for each invocation of the preserve_verify() function.

A direct comparison of the VSSKit2 (32bit) results with the performance results of VSSKit 1 in Table 8.2 indicates a slight decrease in verification performance and a significant slowdown in signature 


\begin{tabular}{|c|c|c|c|c|}
\hline Setup \# & sign & sign/s & verify & verify/s \\
\hline VSSKit2 ST (32bit) & $7.1 \mathrm{~ms}$ & 146 & $7.9 \mathrm{~ms}$ & 132 \\
\hline VSSKit2 ST (64bit) & $3.9 \mathrm{~ms}$ & 256 & $2.8 \mathrm{~ms}$ & 387 \\
\hline Synthetic MT (32bit) & $1.5 \mathrm{~ms}$ & 1901.7 & $5.7 \mathrm{~ms}$ & 490.9 \\
\hline Synthetic MT (64bit) & $0.4 \mathrm{~ms}$ & 6742.2 & $1.1 \mathrm{~ms}$ & 2780.6 \\
\hline \hline
\end{tabular}

Table 8.3: Peak ECDSA NISTP256 latency and throughput values for OpenSSL 1.0.2a on the Nexcom VTC 6201 platform. Test were performed within a single threaded VSSKit 2 and with a synthetic multi threaded benchmark tool that skips all message processing beyond raw signature creation and verification.

creation in version 2 of the VSSKit. Among the major changes between version 1 and 2 of the VSSKit is enhanced support for new signed message formats. This can explain the increase in signature processing times, particularly the creation of more complex signed message formats.

The VSSKit2 (64bit) configuration uses the same setup as the $32 \mathrm{bit}$ configuration, with the major difference of utilizing 64 bit instructions as well as taking advantage of new software optimizations presented in [57]. We observed a performance increase by almost a factor of 3 due to these changes.

The Synthetic MT (32bit) benchmark shows the potential of utilizing all computational resources by fully exploiting the multithreading capabilities of the CPU. The latency for individual signature verification operations is only slightly enhanced over the single threaded VSSKit2 performance. This is solely due to the reduced overhead of raw OpenSSL usage compared to full message processing in VSSKit2. However, the overall throughput measurement demonstrates the impact of multithreading on overall performance. In this setup a total of 8 threads were configured to work in parallel to keep all 4 logical cores busy. The result is an overall throughput of 490 signature verifications per second, instead of an expected throughput of 175 verifications per seconds for single threaded execution. This represents an enhancement of a factor of 2.8.

Finally, the Synthetic MT (64bit) setup shows the overall peak performance achievable on the NEXCOM VTC 6201 on-board unit. The benchmark application was configured to execute 8 threads in parallel, feeding 4 hyper threads on 2 physical cores with work to achieve maximum throughput. With close to 1 millisecond of latency and overall throughput of 2780 verifications per second, it appears realistic that such an on-board unit can provide enough computational resources to sustain acceptable cryptographic performance even under heavy load. 
At least under the assumption that multithreading can be exploited and that the load and calibration of the operating system is adjusted to minimize preemptions and interruptions through context switches, such as those seen in Figure 8.4. 


\begin{tabular}{|c|c|c|c|c|c|c|c|c|c|c|c|c|c|c|}
\hline 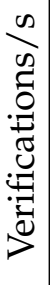 & 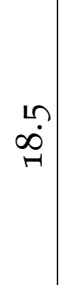 & $\begin{array}{l}\text { in } \\
\text { bे }\end{array}$ & 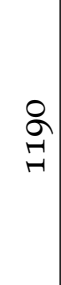 & $\begin{array}{l}\text { à } \\
\stackrel{-}{N}\end{array}$ & $\begin{array}{l}\infty \\
尺 \\
\hat{~}\end{array}$ & ঠి & $\begin{array}{l}\infty \\
0 \\
m \\
\eta\end{array}$ & $\begin{array}{l}\text { ㄴ } \\
\infty\end{array}$ & $\begin{array}{l}\infty \\
\stackrel{\infty}{N} \\
\stackrel{H}{N} \\
\eta\end{array}$ & $\stackrel{\curvearrowright}{\curvearrowright}$ & ڤ & $\begin{array}{l}\text { त̂ } \\
\text { నิ } \\
\text { הે }\end{array}$ & হ) & 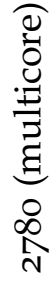 \\
\hline 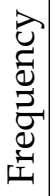 & $\begin{array}{l}\stackrel{N}{\mathcal{I}} \\
\sum_{m}^{m}\end{array}$ & 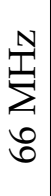 & 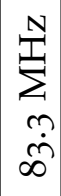 & $\underset{m}{N}$ & $\begin{array}{l}\underset{N}{N} \\
\underset{N}{N}\end{array}$ & $\begin{array}{l}\stackrel{N}{\mathcal{N}} \\
\sum_{0}^{+} \\
\stackrel{+}{+}\end{array}$ & 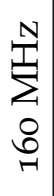 & 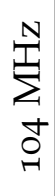 & 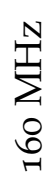 & $\begin{array}{c}\underset{N}{\mathcal{N}} \\
\underset{\mathrm{m}}{\mathrm{m}}\end{array}$ & $\underset{\substack{N \\
\text { m }}}{\stackrel{N}{N}}$ & $\begin{array}{l}\mathbf{N} \\
\stackrel{N}{\mathcal{N}} \\
\stackrel{2}{\mathfrak{N}}\end{array}$ & 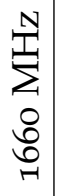 & 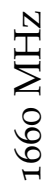 \\
\hline 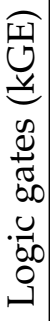 & 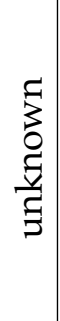 & 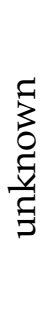 & $\begin{array}{l}\stackrel{1}{\dot{+}} \\
\stackrel{\operatorname{n}}{\sim}\end{array}$ & $\begin{array}{l}0 \\
\dot{+} \\
\stackrel{\sim}{\sim}\end{array}$ & $\begin{array}{c}\Re \\
\stackrel{\leftrightarrow}{ت} \\
\stackrel{ }{\sigma}\end{array}$ & 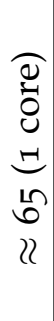 & 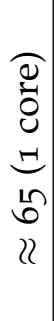 & 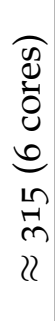 & 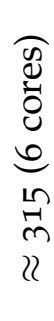 & $\begin{array}{l}\stackrel{-}{N} \\
\stackrel{N}{N}\end{array}$ & $\begin{array}{l}+\underset{n}{\sim} \\
\stackrel{\sim}{N}\end{array}$ & $\begin{array}{l}\stackrel{\sigma}{\dot{m}} \\
\dot{m} \\
\stackrel{n}{1}\end{array}$ & \begin{tabular}{l}
8 \\
8 \\
8 \\
\multirow{+}{+}{} \\
22
\end{tabular} & $\begin{array}{l}8 \\
8 \\
8 \\
+ \\
21\end{array}$ \\
\hline 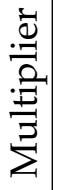 & 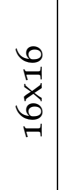 & $\begin{array}{l}\stackrel{v}{\tilde{x}} \\
\underset{\sim}{n}\end{array}$ & 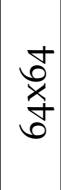 & 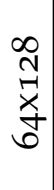 & 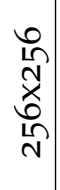 & $\begin{array}{l}\underset{\mathrm{r}}{\mathrm{x}} \\
\mathrm{N} \\
\mathrm{m}\end{array}$ & 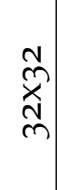 & $\begin{array}{l}\underset{N}{\tilde{x}} \\
\underset{\sim}{n} \\
m\end{array}$ & 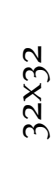 & $\begin{array}{l}\text { ț } \\
\stackrel{x}{\sigma}\end{array}$ & 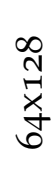 & 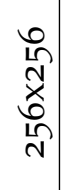 & 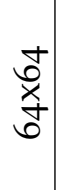 & 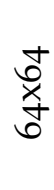 \\
\hline 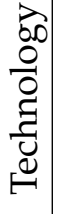 & 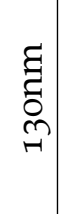 & $\begin{array}{l}\text { ణే } \\
\text { శ్ }\end{array}$ & 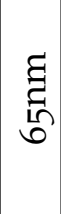 & $\begin{array}{l}\Xi \\
\Xi \\
\text { చొ } \\
\end{array}$ & 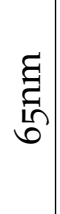 & 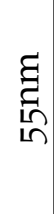 & 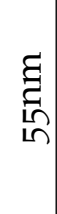 & $\begin{array}{l}\tilde{\Xi} \\
\text { กิ } \\
\text { เก }\end{array}$ & 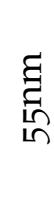 & 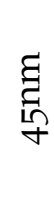 & 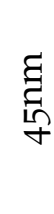 & 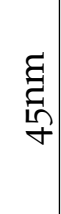 & 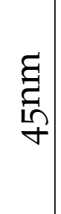 & 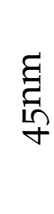 \\
\hline 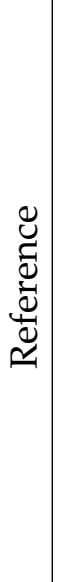 & 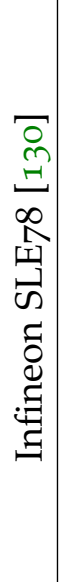 & 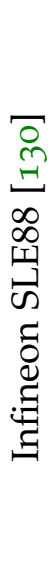 & 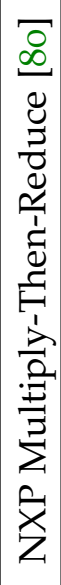 & 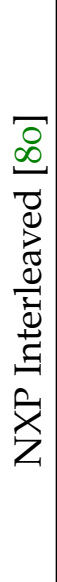 & 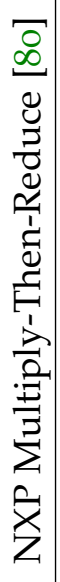 & 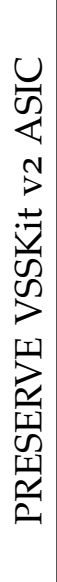 & 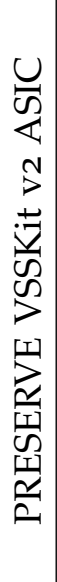 & 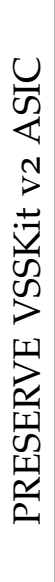 & 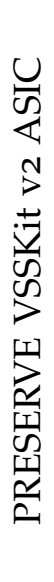 & 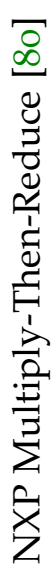 & 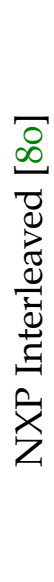 & 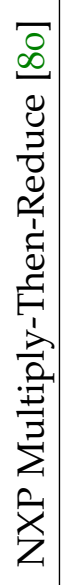 & 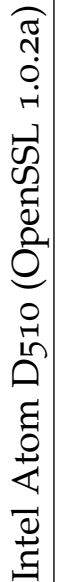 & 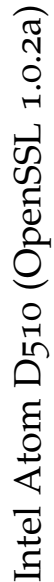 \\
\hline
\end{tabular}




\subsection{CRYPTOGRAPHIC HARDWARE ACCELERATION}

A system that requires only a small set of operations to be performed at very high speed can benefit from offloading such operations to dedicated hardware. This can range from dedicated CPU instructions, to separate co-processor units, and even external devices that perform complex tasks faster than general-purpose processors. This approach can make it possible for an otherwise underpowered platform to fulfill specific performance requirements. Alternatively it can make it more cost effective to meet performance requirements by pairing a lower powered general-purpose system with dedicated hardware.

Acceleration of cryptographic primitives with dedicated hardware is a classic technique and a well known research area. The relevant cryptographic primitive to accelerate for secure $\mathrm{V}_{2} \mathrm{X}$ communication is the verification of ECDSA signatures over the NIST P256 curves. The dominant computationally expensive part of this algorithm is the multiplication of curve points in a given finite field [8o]. Efficient techniques of finite field arithmetic in general and ECC point multiplication in particular have been the subject of numerous studies $[5,8,29,45,61,95$, 120, 136] and entire series of academic events, for example the context of the Workshops on Cryptographic Hardware and Embedded Systems (CHES).

Table 8.4 shows performance attributes for a selection of hardware solutions that might be used to accelerate ECDSA signature verification for security vehicular communication. The design of the multipliers and consequently the amount of logic gates required for a given hardware design are dominating factors for the performance of a solution. The Infineon SLE78 and SLE88 solutions are co-processors for the embedded marked and were not designed to meet the specific performance requirements of secure vehicular communication [130]. These serve only as a baseline for what is commonly available in the general-purpose embedded market. The solutions by NXP were specifically designed to meet the requirements of secure vehicular communication [80]. These designs utilize sophisticated optimizations and large multiplication engines to provide an entire family of ASIC designs that can meet the requirements of secure vehicular communication and could satisfy even higher requirements in exchange for a large consumption of logic gates. The PRESERVE VSSKit v2 ASIC design utilizes much smaller generic multipliers and fewer logic gates. To meet the demands of secure vehicular communication, this design offers parallel executions across multiple ECC cores. Finally, a general-purpose Intel Atom CPU serves as a reference for low power desktop CPU performance. The logic gate count of a general-purpose dual core CPU is not comparable to the gate count of pure ECC cores, but the performance figures indicate that future CPU designs might satisfy the performance requirements for 
secure vehicular communication without the need for dedicated hardware. If multithread operation for the exploitation of parallel execution is used, the existing CPU design is already sufficient. Additionally, the authors of the ECDSA implementation in OpenSSL v1.o.2.a, which was used for the collection of the benchmark values in Table 8.2, indicate that a future iteration of these CPUs might include new instructions that could further enhance ECDSA signature verification performance by $18 \%$ [57].

A slight additional performance penalty should be considered, if data transfer to off-chip solutions is required. However, the overall landscape of hardware acceleration solutions shows that adequate performance can be delivered through dedicated solutions.

\subsection{CONCLUSIONS}

A wide array of benchmarks have been collected in this chapter, which illustrated the software performance of ECDSA signature processing on contemporary on-board units as they are used for vehicular communication in field operational trials. The performance of signature generation was shown to be unproblematic. The previously established performance requirements of 10 to 12 signature generations per seconds could easily be reached by almost all platforms tested in our benchmarks.

However, the performance of signature verification varies widely between the different platforms based on multiple attributes. While the raw clock speed of the compute devices is certainly a factor, we have seen in Table 8.2 that the performance figures do not scale linearly with clock speed across CPU architectures. An example from our selection of tested hardware platform as documented in Table 8.1 illustrates this: The Denso WSU, which is based on a PowerPC platform at 400 $\mathrm{MHz}$, scored higher in all tested performance metrics than the Cohda Wireless $\mathrm{Mk}_{3}$, which is based on a ARM SoC architecture at $533 \mathrm{MHz}$ clock speed. Differences in the Instruction Set Architecture (ISA) as well as secondary effects due to memory access speeds can account for such differences. This initial assessment of secure message processing performance indicated that suitable signature verification performance cannot be achieved with this generation of hardware platforms.

To further investigate the options and attributes that define software performance we looked at parallelism, ISA word size, and implementation quality. In Table 8.3 we selected to set up one of the existing platforms in different ways that allows us to predict the performance of more powerful future on-board units. A switch of the instruction-set architecture to 64 bit word lengths, exploitation of 4 parallel hyperthreads and a change to a new and optimized algorithm implementation yielded significantly faster performance. The implementation 
enhancements consist of a combination of algorithmic optimizations as well as assembly level adaptations to the given Intel ISA. The combination of all these enhancements resulted in 8 times better signature verification latency and an increase of throughput by a factor of 21 . This allows the device to fulfill the requirements of signature verification of 1000 verifications per second.

The configuration to achieve these peak performance calibrations is not representative of systems that we expect to find during the initial deployment of vehicular communication systems. While the NEXCOM VTC 6201 on-board unit is qualified for automotive use, its cost and physical dimensions are larger than those we realistically expect to find in vehicles. However, the direction of technological advancements in mobile and embedded architectures indicates that software implementations will soon be capable of delivering suitable performance, even on smaller hardware. Smartphone platforms have recently started to support 64bit instruction-set variants and high-end models commonly feature multicore designs. It is however necessary to pay attention to performance aspects in the software architectural design of the vehicular communication system.

Finally, we provided an overview of selected hardware acceleration solutions for ECDSA signature verification and observe that the performance requirements for secure vehicular communication can be met by designs with suitable allocations of logic gates.

Our approach to evaluate the current and near term suitability of relevant signature processing solutions was based on an empiric experimental assessment of existing platforms. This was a reasonable method to evaluate current and near future capabilities of software based security solutions for secure vehicular communication. Our results indicate that current OBU platforms cannot deliver the necessary performance. However, it is possible to achieve suitable performance if enhancements in ISA technology and parallel execution can be exploited or if dedicated acceleration hardware is used. 
CONCLUSION

In the introduction of this Part we identified the primary benefits of hardware assisted security in the form of a Hardware Security Module: Secure and accelerated execution of cryptographic functions as well as secure generation and storage of cryptographic key material. In two chapters, we empirically investigated the performance characteristics of accelerated execution on OBU platforms and proposed a novel way of scalable and secure storage of cryptographic key material using PUFs.

In chapter 7 we proposed to use PUFs as a core component in a novel solution that combines the intrinsically secure storage of an physically unclonable function with a key derivation schemes. This enables the generation and storage of large amounts of secret key material within the minimum amount of secure PUF storage. A set of variations of this scheme offers various levels of redundancy and robustness against different attacker classes. We also compared the use of classic secure storage and combinations of key derivation schemes with classic secure storage to achieve similar scalability properties. We concluded that scalable secure storage in PUFs can be a viable alternative to classic secure storage. Cost and availability of effective PUF designs, such as strong PUFs and designs with stable intra PUF responses, are likely to determine the success of schemes like the one we proposed.

Our study of execution performance characteristics in chapter 8 showed that almost all contemporary OBUs would be capable of generating cryptographic signatures fast enough to meet the expected requirements of secure vehicular communication. However, those OBUs alone would not be capable of sustaining the expected maximum throughput of secure vehicular message verifications. Nevertheless, an overview of dedicated hardware designs for accelerated ECDSA signature verification in 8.5 shows an array of solutions that can be used to equip generic OBU platforms with enough additional computational resources to meet the largest expected performance requirement for message verification.

Our investigations also indicate that future OBUs might be capable to deliver satisfactory cryptographic throughput if a set of enhance- 
ments in implementation quality and platform capabilities is met. The main concerns among the implementation quality attributes are the capability to execute cryptographic functions in parallel and the availability of optimized point multiplication routines. If enhancements are combined with an expected increase of OBU platform capabilities, such as extensions of instruction set architectures to 64 bit word sizes and parallel processing designs, we can begin to see that those systems will be capable to provide the necessary performance to meet the requirements of secure vehicular communication.

Our findings show that, in the near term, it is a requirement for secure vehicular communication systems to have a hardware security module for the accelerated execution of cryptographic functions. However, it appears likely that future generations of OBU systems will not require the assistance of special ECDSA acceleration solutions to meet the throughput demands for signature verification. Secure generation and storage of cryptographic key material benefit from having hardware supported randomness and physical tamper resistance properties. PUFs can be an option to provide these attributes, but noisy sensors as random sources and classic secure or encrypted storage can provide cost effective alternatives that do not strictly require a dedicated hardware security module. Secure execution remains as the main property that a general purpose systems cannot offer. Some chip designers are starting to offer trusted execution environments, which are designed to provide isolation of a small secure environment from a more exposed general purposed system. Such solutions blur the line between general purpose systems and system-on-a-chip solutions with an integrated dedicated HSM. Such designs can already be found in contemporary high-end smartphone chipsets and are likely to represent the direction that embedded systems platform with heightened security requirements will converge to. 


\section{Part IV}

CERTIFICATE MANAGEMENT 



\section{0}

OVERVIEW

Two basic constraints define the scalability properties of secure vehicular communication: The processing capabilities of secure messages and the transfer capacities of the communication channel. In the previous chapter, we investigated hardware assisted solutions to enhance the processing capabilities for secure vehicular communication systems. In the following chapters we investigate ways to enhance the scalability of secure vehicular communication through efficient usage of the available communication bandwidth.

In chapter 3.1 we discussed the fundamental requirements and constraints of the cryptographic choices in vehicular broadcast communication. We concluded that digital signatures are the best choice for stateless broadcast authentication. Only permanent reachability of a trusted third party or statefull group formation could enable alternative solutions, which is unlikely to work reliably in the highly volatile network conditions of vehicular communication. Signatures alone however do not ensure trust in the sender. Signatures only provide cryptographic certainty in the authenticity of the sender. To establish trust relationships it is necessary to mark the cryptographic identities of vehicles, represented by the public keys, as trusted.

The solution to this problem is the establishment of key management in a public key infrastructure (PKI) for secure vehicular communication. In a PKI, trust can be delegated from a set of known trusted certificate authorities (CA) to previously unknown entities. Such trust delegation is referred to as certification. In the most succinct setting, this can be expressed though a reconstruction value for an implicit certificate [16]. This only assures that a CA confirms that a given entity, represented by a reconstructed public key, belongs to it's set of trusted subordinates. A more common implementation is a digital signature over a public key. Such an explicit certificate can include an additional codified set of properties and capabilities that the CA grants to the owner of the associated key pair. This could include a validity period of a certificate or special entitlements. In the context of vehicular communication such special entitlements could for example be the right to use emergency vehicle lighting. 
Certificates are a great solution for establishing trust to unknown entities without a requirement for immediate availability of a trusted third party. A short list of trusted certificate authorities, which is distributed a priori, is enough to bootstrap the trust delegation system. However, certificates of unknown entities must be exchanged. In a highly constrained and highly volatile network environment, such as vehicular communication, this can create significant overhead. Careful management of the certificate transfer strategy is required to minimize its impact on the communication channel. A failure to select a suitable strategy could lead to unnecessary congestion of the communication channel. This can ultimately lead to the loss or delay of safety critical messages, particularly in scenarios with high density of vehicles.

The key to an efficient certificate transfer strategy is the realization that trust delegation, and thus the transfer of a certificate, is only required for unknown entities. After the successful establishment of a trust relationship, the trust property and associated entitlements can be cached. Instead of the full certificate it is sufficient to include only a short identifier of the certificate that was used to create a signature. Two fundamental approaches are know from previous works to exploit this property for efficient certificate transfer in vehicular communication:

Calandriello et al. [19] start from the assumption that messages in secure vehicular communication should always include all material that is necessary for the message content to be accepted as fully trustworthy. This implies that a signature and certificates are always concatenated to a message. The concept of an omission scheme is then introduced, which limits the concatenation of certificates to a fixed pattern of omissions and inclusions.

Schoch et al. [128] prioritize the minimization of bandwidth utilization by changing the default assumption. Their approach starts from the assumption that certificates are not included by default. Instead, certificates are only included as necessary. The trigger for certificate inclusion in this scheme is the appearance of an unknown neighbor. This event can be deduced from changes in the neighborhood table, which is a common component in vehicular communication.

The work in this Part considers the fundamental trade-off introduced by such omission schemes: More omissions save more bandwidth and thus reduce the likelihood of losing messages due to congestion in the communication channel. However, omissions unavoidably create a risk of receiving messages that cannot be verified due to missing certificates. This creates a new type of loss of messages, which we propose to call cryptographic packet loss. Our goal is to balance regular packet loss and cryptographic packet loss to achieve an optimal service level for secure $\mathrm{V} 2 \mathrm{~V}$ communication.

In Section II we introduce the concepts and attributes of certificate omission in more detail and present related work in this domain. 
We rely on simulation studies for the majority of our investigations, because no high density deployments of vehicular communications exist. The parameters of our simulations are in line with previous works and are documented in this section as well. Furthermore, we confirm the validity of our simulation model through a comparison with a corresponding analytical model. The main contribution in this section is the insight to connect the negative effects of missing certificates to the positive effects of reducing congestion in a communication channel. We classify these effects as cryptographic packet loss and network packet loss. We use this classification to combine the two effects into a useful unified metric of overall packet loss. In Section 12 we present simulation studies that show the effectiveness of various certificate omission schemes and introduce a novel omission scheme: Congestionbased Certificate Omission. This scheme optimizes for effective noninteractive certificate delivery using network layer knowledge of the load in the communication channel. This way we achieve minimal avoidable cryptographic packet loss, while showing robust scaling behavior in highly congested environments. Section 13 analyzes the impact of different certificate omission schemes and configurations on application level services using Awareness Quality as a high level metric. In the absence of actual deployment of this technology, this is the closest we can come to an evaluation of abstract application impacts of certificate omission. Awareness Quality has the additional benefit of allowing more precise analysis of effects in time, compared to aggregate packet loss statistics. We use the higher granularity of this metric to identify small differences in dissemination performance.

Finally, in Section 14 we introduce the concept of pre-distribution of certificates. This approach deviates from the strict omission and inclusion rulesets and instead considers the problem of disseminating certificates as a challenge that is more similar to caching in general. The idea we propose is to utilize spare bandwidth in the communication channel, which has been freed by certificate omission, to reduce future occurrences of cryptographic packet loss. We identify two basic utility classes of pre-distribution and evaluate their effectiveness in a simulation study. 



\section{1}

\section{CERTIFICATE OMISSION}

Communication between vehicles in the 802.11p wireless communication channel is expected to be secured by pseudonymous signatures, which guarantee the authenticity of messages and provide revocable privacy. Relevant standardization efforts at ETSI [32] and IEEE [63] have proposed specific public key infrastructures (PKI) to provide trust in the authenticity of such signed messages in the context of vehicular communication. In order to verify the trustworthiness of a message, it is necessary to verify a digital signature over a message payload using the public key of the authentic sender. This public key must be directly or indirectly signed by a trusted Certificate Authority (CA) to indicate that the sender is trustworthy. Such a signed public key is commonly known as a certificate. A trustworthy secure message consists of three components: The payload, the signature, and the certificate, as seen in Figure 11.1.

The fact that certificates are directly attached to signed messages is not strictly mandatory. The signature of a message is specific to the hash value of the payload, but the certificate is tied only to the cryptographic identity of the sender. To protect against replay attacks, messages are expected to include unique time stamps, which leads to a different hash and a different signature for every new message. However, the cryptographic identity of vehicles is not expected to change with each new message. As mentioned in Chapter 3.1, very frequent pseudonym changes would interfere with the goal of providing short-term local linkability, which is a fundamental attribute that enables higher level services such as cooperative awareness. Refer to [108] for a survey of pseudonym change strategies and Section 14 for an investigation of a certificate pre-distribution technique that specifically supports the change of pseudonyms.

Under the assumptions that pseudonym changes are rare, the inclusion of certificates is redundant after the trust relationship with a communication partner is established. A significant number of bytes can be saved if the certificate can be safely omitted from secure messages in vehicular communication messages. In place of the full certificate, a system would instead attach a digest or fingerprint of a certificate or 


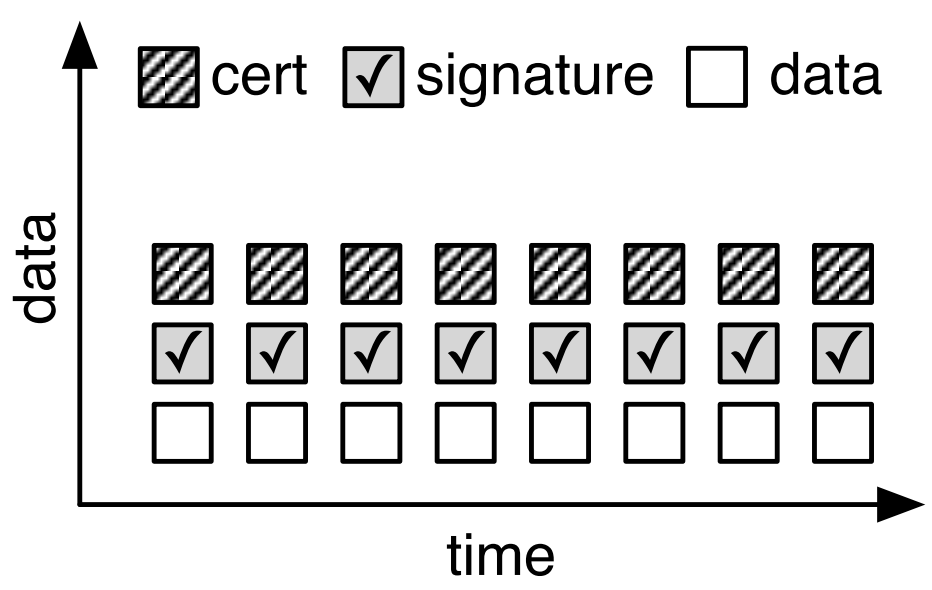

Figure 11.1: Repetition of secure messages in vehicular communication over time. Only the data and signature will change between messages.

public key, which is commonly just a hash value of the full certificate. For simplicity, we refer to the replacement of a certificate by its fingerprint as simply the omission of a certificate. We define certificate omission as the substitution of certificate material, consisting of a single certificate or a chain of certificates, by a shorter representation value. Figure 11.2 shows a conceptual example.

Fingerprints of certificates are a well known feature of PKI systems and commonly seen in PGP/MIME [43] and S/MIME [111] systems. The standardization efforts for vehicular communication already include fingerprints as placeholders for certificates as a specified feature. However, with the exception of CAM messages in ETSI [41], no universal dissemination rules for the distribution of certificates to vehicles are specified in existing standardization efforts.

Quantifications of the respective sizes of certificates, signatures and messages payloads will be presented in the following section. The fact that certificates can represent a significant amount of data makes it attractive to minimize the delivery of certificates while maximizing the service quality. We define service quality of certificate delivery as the ability to fully verify the integrity, authenticity and trustworthiness of incoming signed messages. Ideally, this should be instantaneous for every message. However, for previously unknown vehicles this can require extra communication effort. Waiting periods for exchanges of certificates after the first encounter of a vehicle in communication range need to be minimized. This is the fundamental trade-off that 


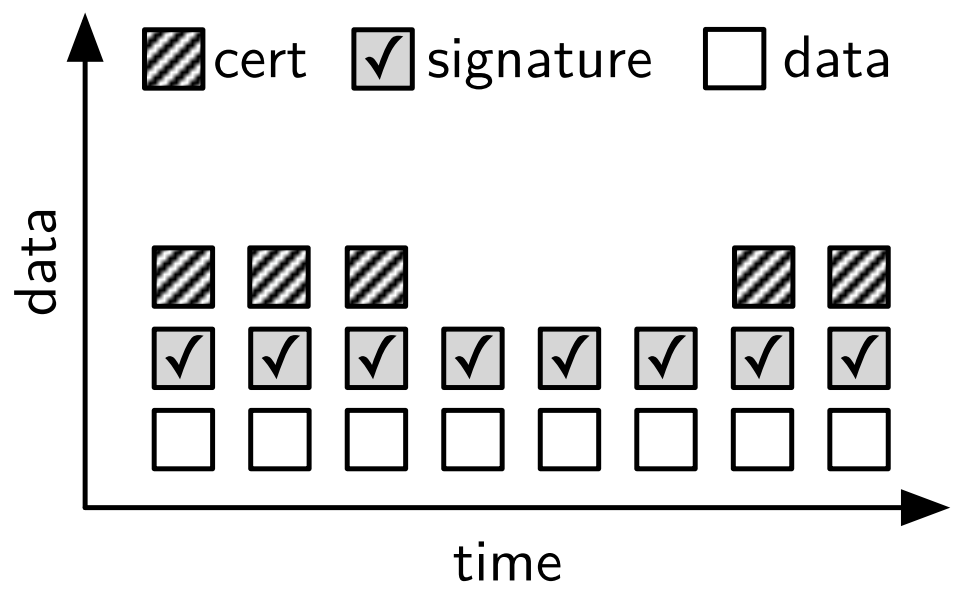

Figure 11.2: Secure message transmission with deliver of some certificates omitted

we investigate in this chapter: What ways can we identify to enhance scalability of secure vehicular communication by reducing bandwidth overhead from certificate transfer without affecting service quality.

\subsection{CHANNEL AND SYSTEM MODEL}

As described in chapter 3.3, our research, especially with respect to communication overhead, is bouned by the requirements of the IEEE 802.11p wireless communication channel [65] for vehicular communication. This channel is in the licensed $5.9 \mathrm{GHz}$ frequency band and is structured into multiple sub-channels. The usage of the sub-channels is regionally different, but there is always one control channel $(\mathrm{CCH})$, which is used for the exchange of the most time-critical message types. As a member of the IEEE 802.11 family of standards, the protocols used for communication are very similar to standard WiFi protocols used for generic wireless communication in unlicensed frequency spectrums. A major difference from the security perspective is the missing concept of a secure association with an access point. The IEEE 802.11 standard expects the establishment of secure communication through Wired Equivalent Privacy (WEP) or the Wi-Fi Protected Access II (WPA2), as specified in IEEE 802.11i.

Vehicular networks are volatile in nature, which reduces the utility of stable associations to specific uplinks. In fact, the concept of uplinks itself does not usually apply to the communication patterns of vehic- 
ular communication. Vehicular communication is expected to consist primarily of broadcast messages to exchange sensor information. The IEEE 802.11p infrastructure is not expected to negotiate a secure channel. Instead, the broadcast messages are expected to be secure on a different level. This omission of secure channel establishment eliminates an extra source of communication overhead for signed broadcast messages. However, the security guarantees of such signed messages should be comparable to the security of messages transferred through a classically established secure channel association. Shifting the security services to a stateless system based on signed messages does provide much improved latency for the encounter of new communication partners. However, the channel itself remains fundamentally unsecured at the level of the medium access control layer.

The distribution of certificates can be very flexible due to the intrinsic guarantees that are provided by the fully authenticated trust chain that they represent. No secure communication channel is required as a prerequisite for the exchange of certificates. This avoidance of a bootstrapping problem for secure delivery of certificates makes it possible to use the plain 802.11p channel for distribution of certificates. Even entirely different out-of-band communication technologies for $\mathrm{V} 2 \mathrm{~V}$ communication such as the cell phone network or optical communication channels could be used for the distribution of certificates. It is uncertain however, if it is realistic to assume presence of alternative $\mathrm{V}_{2} \mathrm{~V}$ communication channel in vehicles.

For the purpose of our studies, we focused on in-band distribution techniques within the 802.11p channels. In fact, we also limited our attention exclusively to one single channel among the 802.11p channels. The use of multi-channel wireless $\mathrm{V}_{2} \mathrm{~V}$ communication is included in the 802.11p specifications and there is active research on open problems in this domain [21]. However, single radio channel switching remains a challenge and it is uncertain, due to the additional cost, if vehicular communication equipment will feature more than one radio unit. The calibration of our selected channel follows the specifications for the 802.11p control channel in general and the regional ETSI ITS G5 specifications [36] in particular. This implies a fixed transmission speed of 6 $\mathrm{MBit} / \mathrm{s}$ and 2odB transmit power.

The bulk of the vehicular communication traffic is expected to consist of position beacons, known as Cooperative Awareness Messages (CAM) in the ETSI family of standards and as Basic Safety Messages (BSM) in the IEEE/SAE family of standards. For our investigations, we use a static model of a typical message type consisting of the three previously identified components: The payload, signature and certificate. The length of the payload was chosen to correspond to the minimal required BSM length of 45 bytes, as specified in SAE J2735 [119]. We do not consider any optional Part II attributes of the BSM format or 
Table 11.1: Message specifications

\begin{tabular}{ll}
\hline Parameter & Value \\
\hline ECC curve & NIST P-256 \\
ECC points & compressed \\
Certificate Size & 140 Bytes \\
Signature Size & 65 Bytes \\
Payload Size & 50 Bytes \\
Broadcast frequency & $10 \mathrm{~Hz}$
\end{tabular}

optional parts of the 1609.2 [63] message format, expect 5 bytes for headers. This leads to a representative payload length of 50 bytes. The size of signatures and certificates is defined by the parameters of the selected cryptographic primitives. The security services specified in IEEE 1609.2 offer different options for these cryptographic primitives. From these options we selected the compressed representation of ECDSA signatures over the NIST P-256 curve. We do not consider certificate chains in our studies, but we note that certificates chains would increase the benefit of certificate omission, as the cryptographic additions would get even larger. The sizes of the cryptographic message components under these choices are 64 bytes for the ECDSA signature and 140 bytes for the certificate. A certificate consist of at least one signature and one public key, as well as optional headers and serialized descriptions of additional authorizations. In our example we assume 64 bytes for a signature and a public key, plus 12 bytes for headers and authorizations. The overall size of one representative secure message including certificate is therefore 255 bytes and only 115 bytes if the certificate is omitted. Assuming a replacement of the certificate by a CertIDio of 10 bytes length, we see that a certificate omission saves roughly 50 percent of the size of a secure message. The broadcasting frequency is calibrated to $10 \mathrm{~Hz}$, as suggested by SAE J2735 [119]. A summarized description of the standard message specifications is presented in Table I1.1.

The certificate of a sender does not necessarily have to be transmitted by the same sender. Other neighboring vehicles could send certificates for another vehicle, even if those other vehicles are not trusted yet. We will discuss inclusion of third party certificates in Section 14. Since third parties are by definition not directly involved in the communication between two parties, it is difficult to efficiently manage the delivery of certificates by third parties. Explicit requests could be used to indicate interest in certain certificates by specific vehicles, and thus control the 
Table 11.2: System model assumptions

\begin{tabular}{ll}
\hline Parameter & Value \\
\hline Communication medium & 802.11p under ETSI G5A rules \\
Certificate request indication & Passive \\
Certificate distribution & in-band \\
Certificate forwarding & Single-hop
\end{tabular}

delivery of certificates. However, such messages consume bandwidth as well, which counters the whole idea of omitting certificates for the purpose of saving bandwidth. Furthermore, we have to consider that requesting a missing certificate through a round trip of requests and responses introduces a significant amount of delay when a previously unknown vehicle is encountered for the first time. We will refer to this problem as the first encounter problem.

Dissemination of certificates is also possible over multiple hops, either through predictive inclusion or triggered by certificate request messages that are carried over multiple hops. However, the trade-offs of saving bandwidth through certificate omission and using additional bandwidth for certificate request messages already lead to diminishing returns. These techniques combined with multi-hop certificate delivery will result in ever more diminishing returns in bandwidth savings and more unfavorable delays until verifiability of secure messages. Future works might want to consider multi-hop distribution techniques and the use of explicit certificate request messages; however we focus our studies on single hop distribution and direct inclusion and omission of certificates.

A summary of all system model assumptions is shown in Table 11.2.

\subsection{RELATED WORK}

Based on the previous definition of the assumptions and the scope of this work it is useful to discuss relevant related work in this area.

A central assumption in this work is the idea that a receiver of a message must either discard it, or delay processing of the message until reception of the certificate that belongs to the signer of that message. However, although there is clearly a risk of accepting malicious inputs into a vehicles data processing systems, accepting unverifiable messages might be justifiable in practice. Techniques have been proposed to omit the verification of signatures if a system can safely assume 
that there is no plausible reason to assume that an attacker might have manipulated the data [128]. This could be the case if the behavior of another vehicle remains within a given set of context-based extrapolations. This would not be applicable to the first messages received from unknown vehicles. Furthermore, an attacker could introduce deviating information successively while staying under a given threshold to not trigger a demand for signature verification. Additionally, an attacker could also exploit this behavior to cover up noteworthy events by creating messages that wrongly advertise the absence of deviating behavior. Such maliciously normal messages would create false negative results and would be accepted by the receiver because verification would be skipped. Due to these concerns, we adhere to this strict operational assumption of discarding unverifiable messages throughout this research.

The basic distribution problem of certificates is a well known issue for all public key infrastructure (PKI) system. As a topic within the larger area of key management, it is among the major challenges that makes deployment of cryptography difficult in practice. The largest operational PKI is the informal agglomeration of trusted certificate authorities that issue X.509 certificates for use in the public internet. Internet links are typically less limited by bandwidth concerns and it is commonly assumed that certificates can be delivered during the initial handshake protocol of secure connection setups. This is the solution that is used for direct SSL/TLS [137] connections. In the case of indirect communication, such as the delivery of email messages, it is expected that any necessary certificate material is included in the message. This is the distribution method preferred by the S/MIME[111] secure messaging system, which can be applied not just to email but essentially to any message oriented service, e.g. for the security of session description messages for the initiation of real time streams [44].

Another option, which is allowed by the aforementioned S/MIME system and preferred by the alternative PGP/MIME [43] system, is the simple lookup of relevant certificates at a third party server. This approach is very suitable in highly connected networks with low latencies and does not require a significant amount of trust into the third party server that distributed the certificates, as the certificates should intrinsically provide the trust chain up to a trusted root. However, the communication round trip to a dedicated third party service makes this approach not directly applicable to $\mathrm{V}_{2} \mathrm{~V}$ communication systems. Availability and latency requirements do not allow a strict reliance on remote backend services.

Research about specific ways to implement efficient $\mathrm{V}_{2} \mathrm{~V}$ exchange of certificates was effectively initiated by concerns about the scalability of secure $\mathrm{V}_{2} \mathrm{~V}$ message exchange. The European $\mathrm{FP}_{7}$ project SeVeCom in particular has spawned a number of research contribu- 
tions that investigated the cost of adding security and privacy to $\mathrm{V} 2 \mathrm{~V}$ communication [19, 73, 98, 112]. This research resulted in concrete proposals of certificate distribution with periodic omissions. A certificate omission scheme based on neighbor events with the goal of maximizing omissions was proposed in a follow-up to the efforts of the SeVeCom project [128]. These work represented the state-of-the-art at the beginning of 2011.

Meanwhile, the standardization efforts created architectures that at least foresee certificate omission as a feature, even if no specific omission algorithms have been documented. In Annex B2.2 of IEEE 1609.2 v2 D12 [63] a TimeBetweenFullCertChainTransmissions attribute is documented, which indicates that the full certificate chain could at least be truncated. The presence of a certificate digest format implies that certificate data could be omitted entirely. The same document recommends that an entity that sends time-critical data should send a full trust chain of certificates at least one time per second.

Similarly, the ETSI family of standards for vehicular communication has included provisions to enable omission of certificates. However, a larger focus has been set on reducing the frequency of messages in the channel altogether, as part of Decentralized Congestion Control [40]. In this context, ETSI has issued recommendations that are very specific to message types. Cooperative Awareness Messages (CAM) in particular are subject to two technical specifications. Annex B of [39] defines an adaptive message generation frequency adjustment based on a set of environmental conditions such as deviations from an extrapolated trajectory and changes in vehicle speed. The rate of CAM traffic is only bound to between $\mathrm{rHz}$ and $10 \mathrm{~Hz}$ frequency. Additionally, the ETSI security and headers specification for vehicular messaging [41] foresees a special certificate omission protocol, which applies exclusively to CAM traffic. The specifications allow for an event based certificate omission technique in combination with a fallback to include a full certificate chain at least once per second, as seen in the IEEE 1609.2 recommendation above. The ETSI specifications for CAM security went one step further and also specified an explicit certificate request message to actively seek the transmission of a missing certificate.

Explicit certificate request messages that are not sent to a remote backend service, but are instead sent to surrounding vehicles in communications range, represent a fallback option that was not considered in previous research. Schoch et.al. [128] have considered event based reaction mechanisms, but did not investigate a solution with dedicated certificate request messages. An obvious problem of explicit certificate request messages is the fact that such messages create additional traffic in the same communication channel where the certificate omission technique is used to reduce traffic. Furthermore, it is conceivable that such a feature could be abused as a traffic augmentation component in 
a denial of service attack. Considering these explicit certificate request messages requires careful consideration of these aspects, which was out of scope in the context of our work. A recent research effort [11, 12] is beginning to investigate these topics. Bittl et al. have simulated various refinements of implicit and explicit certificate request schemes, as suggested in [128] and [41]. The results show that aggressive certificate exchange settings reduce the amount unverifiable packets, with no certificate omissions naturally eliminating all unverifiable packets. However, their simulations also showed that the additional bandwidth consumption did not lead to any negative effect in their chosen scenario, which did not suffer from channel congestion. This was true even when considering an attacker attempting a traffic augmentation attack through excessive certificate inclusion through unsigned certificate requests.

Another feature that was added as an option in the standardization process of IEEE 1609.2 are implicit certificates, such as the Elliptic Curve Qu-Vanstone Implicit Certificate Scheme [16]. This technique allows the reconstruction of a certified public key from the public key of a certificate authority, a chosen identifier, and implicit certification data. This can save a considerable amount of bandwidth, although it does not remove the need to exchange certification data entirely. A related kind of compression effect could be achieved through exploitation of network coding techniques such as Fountain or Erasure codes, which have been proposed in the area of certificate revocation list distribution in VANET [100]. We consider the issue of such encoding techniques to be orthogonal to the question of sending certificate material.

A different area of research considers alternative trust and authentication methods that do not require the exchange of any explicit certificates or shared secrets. Identity-based signatures have been proposed for use in VANET [71] and could eliminate the need for certification altogether. A shift to attribute-based anonymous authentication [20] could similarly eliminate the need for certificates. However, operational aspects such as the speed of cryptographic primitives make directly usage of these schemes unsuitable for applications in pure vehicular adhoc networking. See 3.4 and 8 for discussions of relevant performance requirements.

Hybrid schemes that use a mix of pseudonymous certificates and alternative signature schemes have been proposed in recent publications. In[53] it is suggested to insert attributed based authentication into the $\mathrm{V} 2 \mathrm{~V}$ broadcast authentication domain at the pseudonym issuance stage. This can effectively weaken revocable privacy, which enhances anonymity, and avoids the performance penalties of using attributed based cryptography by continuing to use pseudonymous certification for the $\mathrm{V} 2 \mathrm{~V}$ broadcast. Another hybrid scheme was proposed in [19], which would use groups signatures for the issuance of pseudony- 
mous certificates in privacy enhancing groups formations. Such hybrid schemes could be a promising additions to broadcast authentication with pseudonymous certificates, if better anonymity of prioritized over revocable privacy. However, these schemes do not replace certificate based broadcast authentication.

A further option is to involve the assistance of road side units (RSU) to mitigate performance and key management problems [25, 145, 146]. The RSU could perform computationally intensive tasks on behalf of vehicles and allow them to switch to faster symmetric key authentication. However, the uncertain availability and reachability of such supporting infrastructure precludes practical consideration of such proposals outside of urban scenarios.

The certificate distribution problem is deeply connected to the usage of a PKI system and is unlikely to disappear entirely. Clever encoding schemes, keeping certificate chains short, or the availability of alternative communication channels could reduce the cost of certificates distribution. However, the fundamental problem of requiring a trust delegation mechanism that can work without online access to a trusted third party will remain. The previous state of the art in $\mathrm{V}_{2} \mathrm{~V}$ certificate distribution is centered around two omission approaches: Periodic Omission $[17,98]$, which is a rigid but reasonably straightforward approach, and Neighbor-based Omission [73, 128], which optimizes for the absolute maximum justifiable omission through an event based certificate inclusion approach. These systems have been evaluated predominantly through bandwidth consumption and packet loss metrics.

In the following chapters we will show that assessing the effectiveness of certificate omission cannot rely solely on measuring the amount of saved bandwidth. In the next section we motivate the need for an adaptive certificate omission scheme that strikes a balance between bandwidth savings and avoidance of unverifiable packets. A simplified analytical model indicates that there are little gains to be achieved from certificate omission in uncongested scenarios, which was not considered sufficiently by previous certificate omission proposals. Also, by separating network packet loss effects and packet loss due to cryptographic unverifiabilty, we can show the exact negative influence of omitting certificates too aggressively. Minimizing these negative effects is especially critical during the first encounter of an unknown vehicle and generally more important than bandwidth savings. A reduction of emergency braking distance due to these negative effects has been used as a small case study in [19]. Nevertheless, this aspect has not been considered enough in previous proposals. We extend this approach further in Section 13 and use Awareness Quality [124] as a fine grained metric that allows us to further investigate the properties of certificate omission schemes. 
The introduction of messages that cannot be verified due to the absence of a certificate based trust relationship is the main design limitation of certificate omission. The design goal of certificate omission on the other hand is bandwidth savings to reduce the likelihood of packet loss. To analyze the effect of certificate omission we consider the combined effects of reduced packet loss due to bandwidth savings and introduced packet loss due to unverifiability. To differentiate between the two types of packet loss we will refer to unverifiable messages a Cryptographic Packet Loss (CPL). The classic types of packet loss due to network effects will be referred to as Network Packet Loss (NPL). These networks effects in the wireless communication channel will be collisions of wireless signals, buffer overruns, and packets that expire due to queueing in buffers while the channel is in use.

Investigating the scalability attributes of CPL and NPL under real conditions would require the availability of large deployments of vehicular communication infrastructure. Vehicular communication has however not yet reached a stage of commercial deployment to end users. Field Operational Test (FOT) projects do exist, but none of these experimental deployments are large enough to achieve a connectivity rate that would allow investigations of realistically congested channel usage. The use of simulation models is the only option for meaningful pre-deployment investigations into the scalability behavior of vehicular communication. Simulation models enable us to analyze the highly dynamic interactions of vehicle mobility patterns, wireless communication effects, and higher-level protocol behavior. Trying to capture the combination of all these effects in a unified purely analytical model would be prohibitively complex. We do validate our simulation against a simplified theoretical model, but we rely on existing simulation tools and established simulation scenarios for our core investigations.

Our selected simulation package is the JiST/SWANS[4] environment with the UUlm software extension package[72]. Signal collision effects in this simulator are realized through an additive noise model of the radio channel on the receiver side. The signal processing thresholds for collision detection follows the 802.11 energy parameters. Buffer effects are modeled through finite queues on the sender side. The buffer allocation is set to 50 slots, which is enough for a 5 second timeout at the predefined static sending rate of $10 \mathrm{~Hz}$. A summary of the calibration settings of our simulation infrastructure is shown in Table 11.3. The simulation environment provides 802.11p radio simulation and a realistic vehicular mobility model called STRAW [26], which uses map data from the U.S. Census Bureau. This simulation package allows us to efficiently simulate scenarios with a high density of vehicles [129]. We generally use a $3 \mathrm{~km}$ by $3 \mathrm{~km}$ urban city map in 
Table 11.3: Simulation parameters

\begin{tabular}{ll}
\hline Parameter & Value \\
\hline Field size & $3 \mathrm{~km} \times 3 \mathrm{~km}$ \\
MAC & $802.11 \mathrm{p}$ \\
Fading & Rayleigh \\
Pathloss & Two-ray ground \\
Noise & Additive \\
Simulation time & $60 \mathrm{~s}$ \\
Beaconing frequency & 10 Hz \\
Payload Size & 50 Bytes \\
Number of nodes & 100,..., 1300 \\
Node placement & STRAW [26], \\
Node mobility & STRAW [26],
\end{tabular}

Suffolk County, U.S.A., which is the same scenario as used in previous research in omission schemes [48, 128].

At the beginning of this section we highlighted that only simulation allows us to efficiently combine all the complex aspects of vehicle mobility patterns, wireless communication effects, and higher level protocol behavior into a coherent experiment. Here we give a sketch of an analytical model that captures basic wireless channel properties and simplified omission protocol behavior into a formal model. Both aspects have been abstracted to keep complexity low. The development of this model serves to demonstrate the underlying effects when modeled in an abstract analytical way. The behavior predicted by these abstract models motivates the need for more detailed simulation studies.

Network packet loss generally depends on a multitude of factors. Such factors can be the distance between sender and receiver, the payload length of messages, the load on the communication channel, shadowing and reflection of signals, or environmental aspects such as line of sight. To remain independent of the intricacies of signal propagation details in specific scenarios, we can restrict our assumptions about the communication channel to an abstract packet delivery probability function $D_{s}(d)$ for a given scenario $s$ with the distance $d$ between sender and receiver as the only variable input. This function incorporates averaged consideration of aforementioned attributes such as payload length and channel load, including the averaged effects of the selected omissions scheme on these attributes. While detailed sim- 
ulations are invaluable tools for the evaluation of omissions strategies, we want to confirm that an analytical model would show the same effects in a more abstract way. We use this abstraction to validate the general independence of certificate omission effects from the details of a given simulation. Relying only on the averaged macroscopic effects of omissions should show the same effects as our simulation setups.

Certificate omission achieves lower NPL through a reduction of load in the communication channel. This is a trade-off against potential CPL. However, this CPL effect is only present until the first reception of the certificate of a sender. Therefore, we want to quantify the time until the first reception of the certificate of a vehicle with an unknown cryptographic identity.

The predominant communication pattern in vehicular communication is expected to be the exchange or position beacons, known as CAM or BSM in the ETSI ITS and IEEE 1609 families of standards. These beacons are expected to be sent at a fixed maximum frequency of $1 \mathrm{oHz}$. We use this fixed schedule to establish a baseline metric of beacon periods, which represents 100 milliseconds of time. This beacon period allows us to analyze network effects based on rounds of message exchanges.

Finally, we combine the model of the expected time until the first reception of a certificate $(\mathrm{CPL})$ with the model of general NPL packet loss. This yields the packet reception probability at the receiver side under consideration of CPL and NPL for a packet delivery probability $D_{s}(d)$ as a function of beacon periods and distance between sender and receiver.

We select two representative examples of $D_{s}(d)$ based on the packet delivery properties of the 802.11p subsystem in the JiST/SWANS simulation package with extensions from the University of Ulm. We use two scenarios to illustrate our analytical model:

- No congestion: A scenario with two vehicles communicating in a 802.11p channel with default settings. No other vehicles introduce noise or packet loss in this scenario. Only signal propagation effects reduce packet delivery success over longer distances between the two vehicles.

- High congestion: A scenario that uses a backdrop of a high load on the communication channel, produced by vehicles using the same communication pattern as the sender and receiver vehicles. By projecting a $1 \mathrm{~km}^{2}$ scenario with 260 vehicles on a $\sqrt{2} \mathrm{~km}$ one-dimensional line we replicate a simplified high congestion scenario as suggested in [126]. We extend the scenario in each direction by the length of the maximum sensing range with the same vehicle density to ensure equal congestion in the center of the scenario. 
Figures 11.3 and 11.4 show the packet delivery rates for the selected low and high load simulation scenarios. Having different certificate omission schemes as part of the scenario results in different channel congestion levels. This is due to differing lengths of messages causing diverse rates of packet loss. We use simple periodic omission schemes in these scenarios, which include a certificate once within a given period of $n$ beacon cycles, e.g. $\mathrm{POoC}_{3}$ includes a certificate once within three beacon cycles[17]. The NoOm scheme performs no omissions and serves as a baseline. Figures 11.5 and 11.6 show polynomial curve fitting applied to the simulated values to arrive at smooth distribution curves. This represents the baseline for packet delivery success (NPL only) in our examples.

The delivery rate function $D_{s}(d)$, and thus the NPL model, do account for the effect of varying payload sizes in a scenario. As such, the scenario selection $s$ adjusts the overall packet delivery probability. This is an abstraction of all channel utilization effects, including payload length variances due to the selected omission scheme. Next, we want to derive the explicit probability of successfully receiving a message with an included certificate, mc. We derive this from the overall message delivery success rate multiplied by the rate of certificate inclusion, which we introduce as the discrete constant value $c$.

$$
\operatorname{mc}(\mathrm{d})=\mathrm{D}_{\mathrm{s}}(\mathrm{d}) * \mathrm{c}
$$

For example, in the case of $\mathrm{POoC}_{3}$ we omit the certificate two times followed by one inclusion, leading to an inclusion rate of $1 / 3$. Figures 11.7 and 11.8 show the resulting graphs for the example scenarios and the investigated omission schemes. The graph of NoOm is unchanged compared to the previous figures, as the certificates are always included.

As the probability of receiving a certificate is known, we can derive the probability of reception of a certificate within a given number of beacon periods. This defines the extent of CPL that is introduced by certificate omission. We consider the probability of receiving a certificate within any of up to $n$ beacon periods as the inverse of the probability to not receive any certificate within $n$ beacon periods. We start with the probability of not receiving a certificate within one beacon period $\mathrm{mn}$, which is the inverse of receiving a message with a certificate.

$$
\operatorname{mn}(d)=1-D_{s}(d) * c
$$

Taken to the power of $n$, we get the probability that no certificate is received in any of $n$ beacon cycles. The inverse of this is then the probability that a certificate is included and successfully received at 
least one time in $n$ beacon cycles. Thus, we get the probability $c d$ of having received a certificate within $n$ beacon periods as

$$
c d(d, n)=1-\left(\left(1-D_{s}(d) * c\right)^{n}\right)
$$

We now have two free variables - $d$ and $n$ - and constant choices of certificate inclusion rate $c$ and scenario $s$ for packet delivery success.

Figures 11.9 and 11.10 show $c d(d, n)$ as two-dimensional graphs with $d$ fixed at $d=300$ and several choices of omissions schemes. The choice of omission scheme influences the graphs, as before, through the scenario selection $s$ and the certificate omission rate $c$. The selection of $d=300$ meters as an interesting distance is based on the use of this limit as the delimiter of a safety relevant risk zone around a vehicle [125]. We clearly see in this visualization, how the probability of having received a certificate approaches $100 \%$ as the number of beacon cycles $n$ increases and how this is dependent on the selection of the certificate omission scheme.

Finally, we can now combine the probability of having received a certificate (CPL) with the probability of receiving a packet at all (NPL). The multiplication of these probabilities gives the overall probability of successful packet transfer under consideration of both sources of packet loss, cb.

$$
\mathrm{cb}(\mathrm{d}, \mathrm{n})=\left(1-\left(\left(1-\mathrm{D}_{\mathrm{s}}(\mathrm{d}) * \mathrm{c}\right)^{\mathrm{n}}\right)\right) * \mathrm{D}_{\mathrm{s}}(\mathrm{d})
$$

Figures 11.11 and 11.12 show the overall packet delivery rates $\operatorname{cd}(\mathrm{d}, \mathrm{n})$ for the two load scenarios and subselections of omissions schemes in the scenario, at the reference distance of $300 \mathrm{~m}$ between sender and receiver. In Figure 11.11 we see how all schemes converge to the same ideal packet delivery success rate if there is no congestion. Certificate omission in this scenario only introduces downsides without any improvements. Figure 11.12 however shows more diverse results. Certificate omission schemes converge to different packet delivery rates after the initial probability of CPL subsides. The speed of reducing $\mathrm{CPL}$ and the convergence to higher overall packet delivery success values give certificate omission schemes a clear advantage over not performing certificate omission. We can see the same effect in Figure 11.13 and Figure 11.14. Here the packet delivery success rates converge to much higher levels of delivery success, only due to the closer distance between vehicles. This is expected, since the ultimate packet delivery success after the elimination of CPL is bounded by the natural NPL in a crowded communication channel. As we can see again, this bound varies with the specific load properties of a given scenario, in particular with the selected omission scheme and the distance between vehicles. 
Special attention should be paid to the NoOm configuration, which performs no omissions at all. Under load, this configuration always converges to a significantly lower packet delivery success rate. Even during the initial beacon cycles, where CPL is a relevant factor, it is beneficial to have low levels of certificate omission. Not performing any omissions only appears to yield an advantage at a very close distance, such as 10om, and only during the first beacon cycles. However, considering the mobility patterns of moving vehicles it is unlikely that a vehicle is still missing certificate material when it reaches such close proximity.

In this section we developed a simple analytical model to predict packet delivery probabilities for secure broadcast communication in vehicular networks under consideration of certificate omission and cryptographic packet loss. Through variations of the abstract packet delivery probability function $D_{s}(d)$ we could observe and quantify the negative impact of certificate omission on CPL due to missing certificates. We could also show the benefits of a reduction of NPL due to the bandwidth savings of certificate omission. However, these benefits only appear if there is high load in the communication channel.

The abstractions that have been used in this section oversimplify certain attributes of the certificate delivery process. The investigated schemes are simplistic and not modeled in a stateful way. The delivery rate function $D_{s}$ subsumes a large number of effects into a mean delivery success rate, which only depends on a single free variable d. More detailed considerations of packet length could enhance the quality of the model. Also the influences of vehicle placement and mobility, as well as more realistic propagation models, have been abstracted away into the scenario dependent delivery success function $\mathrm{D}_{\mathrm{s}}$.

In the following sections we do not proceed to address these limitations through larger analytical models. Instead, we move on to use simulation environments to efficiently study the effects of complex behavior in much more detail. We will use well-studied simulation implementations of the relevant vehicle mobility patterns, wireless communication effects, and higher level protocol behaviors. Nevertheless, the abstract model of certificate omission effects revealed that sustained improvements in packet deliver success are likely to be achievable through certificate omission. The model also revealed that certificate omission in an uncongested channel does not offer benefits, but introduces a considerable potential for CPL. We use this as a strong motivation to further investigate the trade-offs of certificate omission, especially during the period of the first message exchanges. In the next section we will proceed to apply the insight gained in this section; there is no benefit to certificate omissions if the communication channel is not under heavy load. We do this by proposing an omissions 
scheme that adaptively scales the omission rate based on the load in the communication channel. 


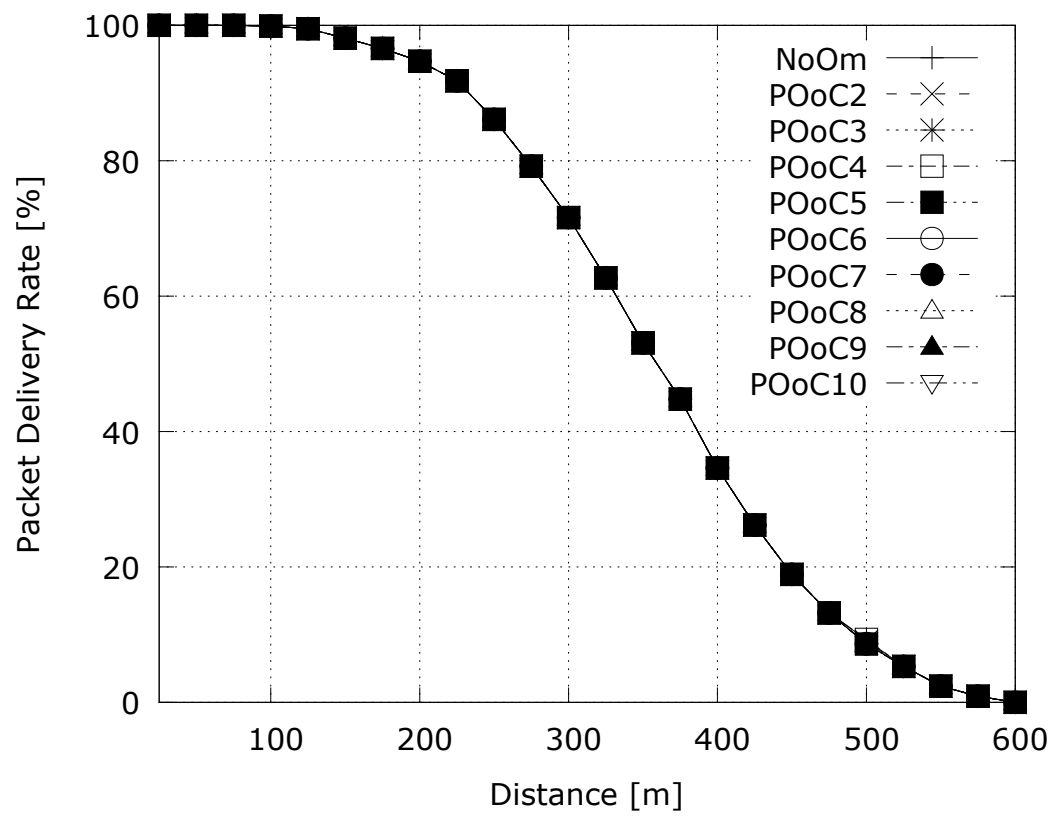

Figure 11.3: Packet delivery rates in scenarios without load in the communication channel follow ideal algorithmic probability patterns

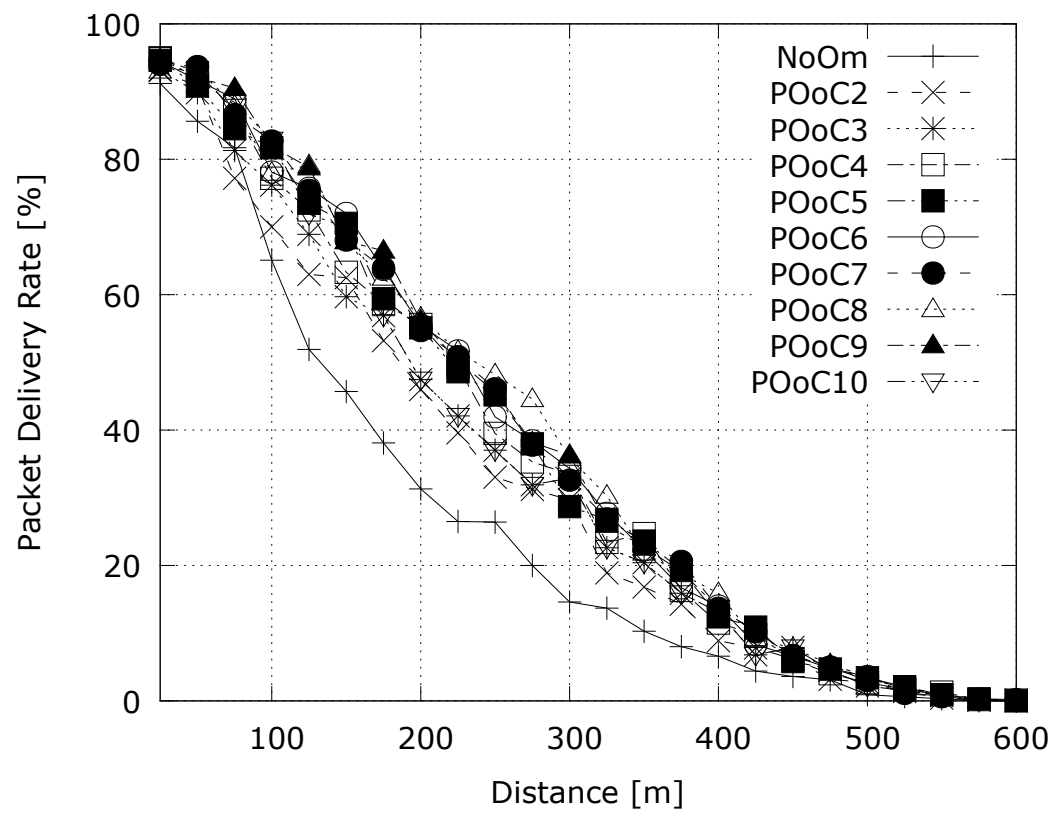

Figure 11.4: Packet delivery rates in scenarios with high load in the communication channel suffer from diverse rates of packet loss 


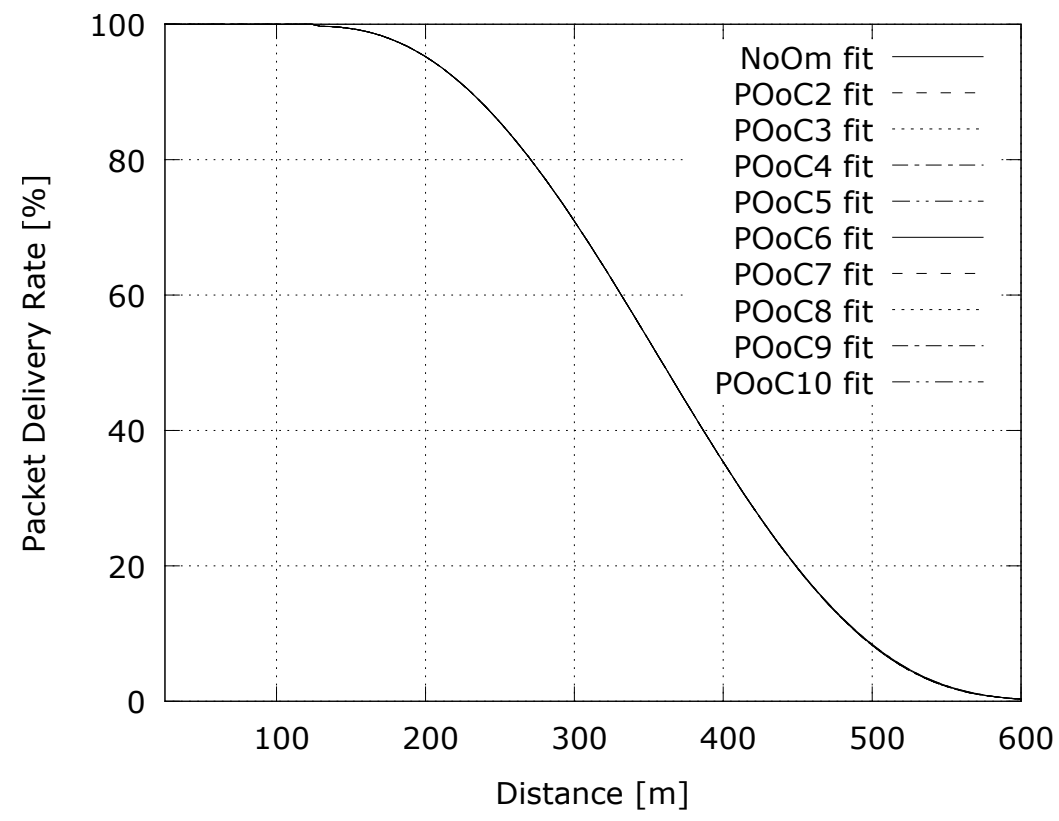

Figure 11.5: Polynomially fitted packet delivery rate without load in the communication channel

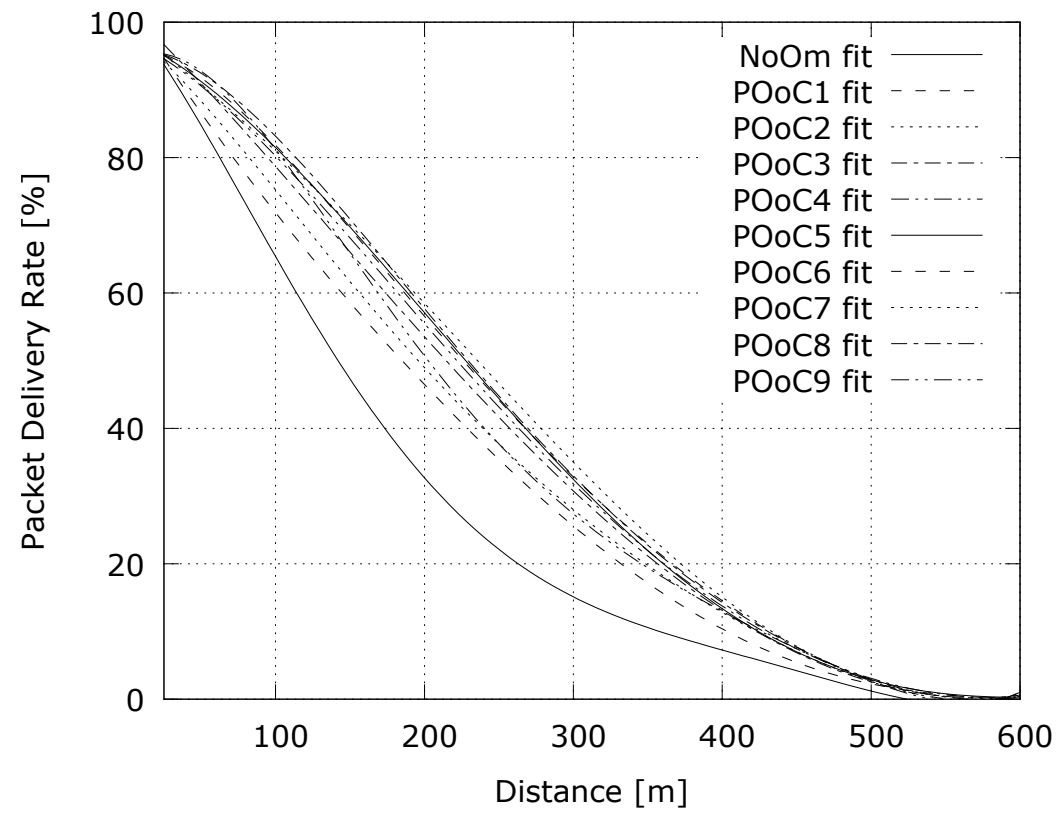

Figure 11.6: Polynomially fitted packet delivery rate with high load in the communication channel 


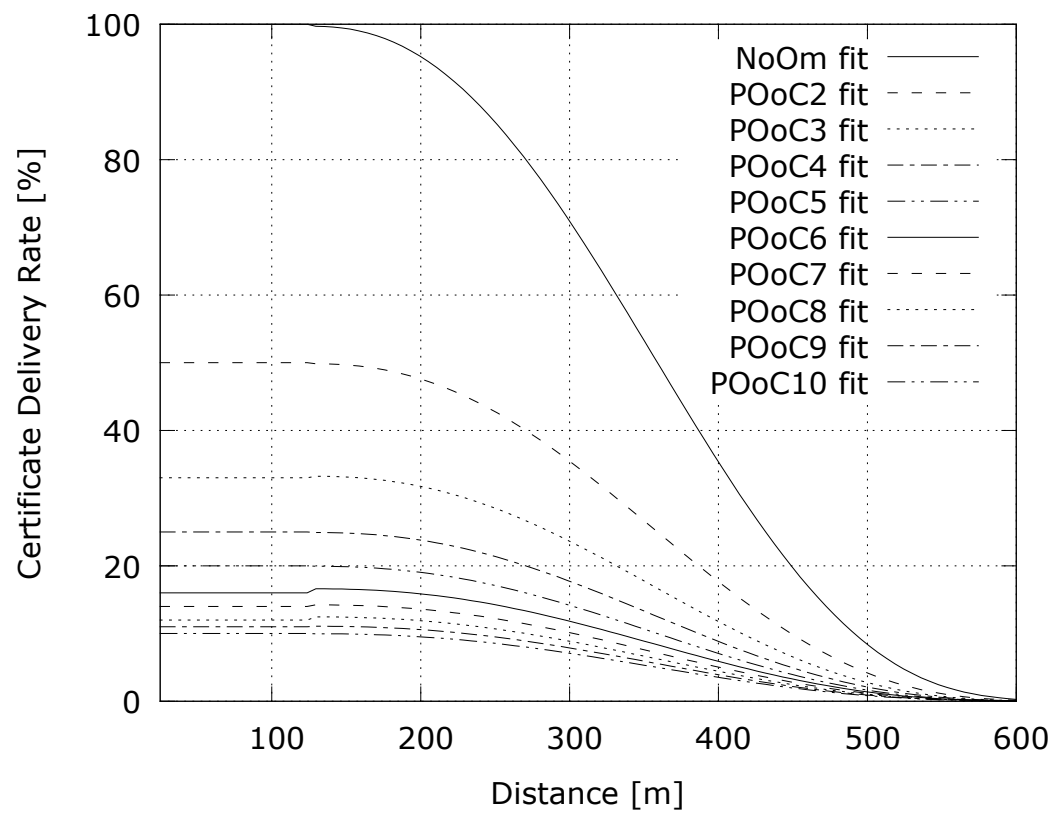

Figure 11.7: Certificate delivery rate $\mathrm{mc}$ without load in the communication channel

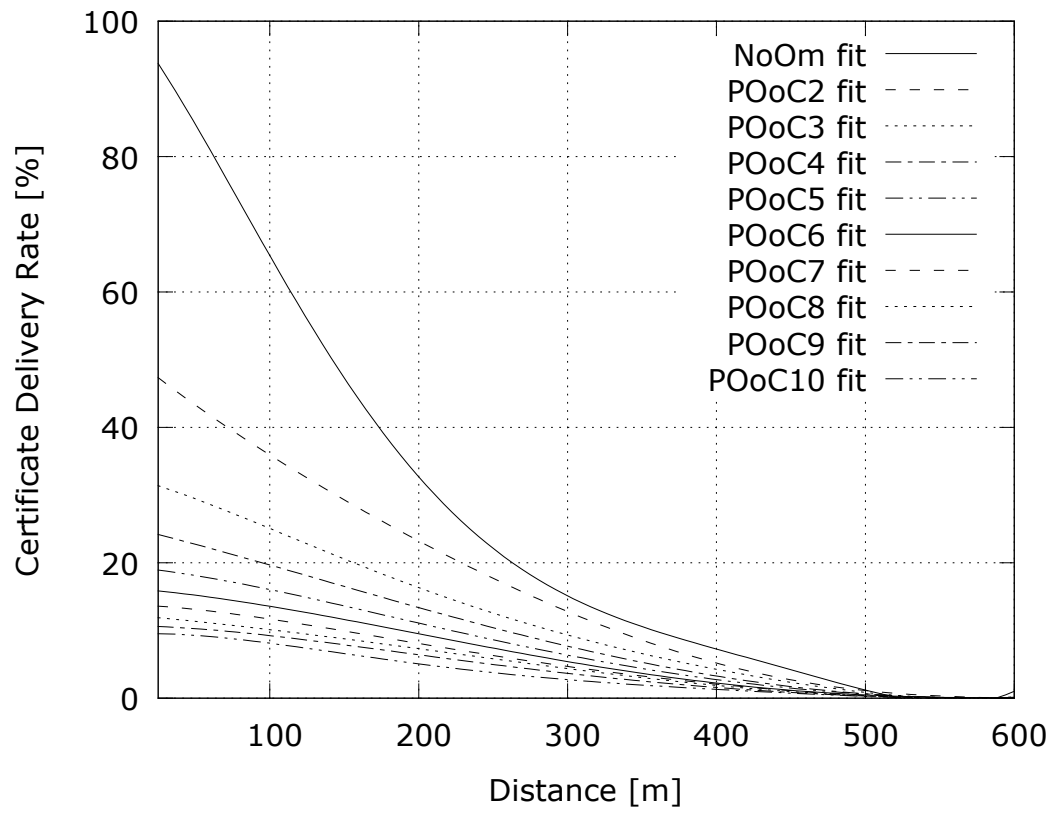

Figure 11.8: Certificate delivery rate $\mathrm{mc}$ with high load in the communication channel 


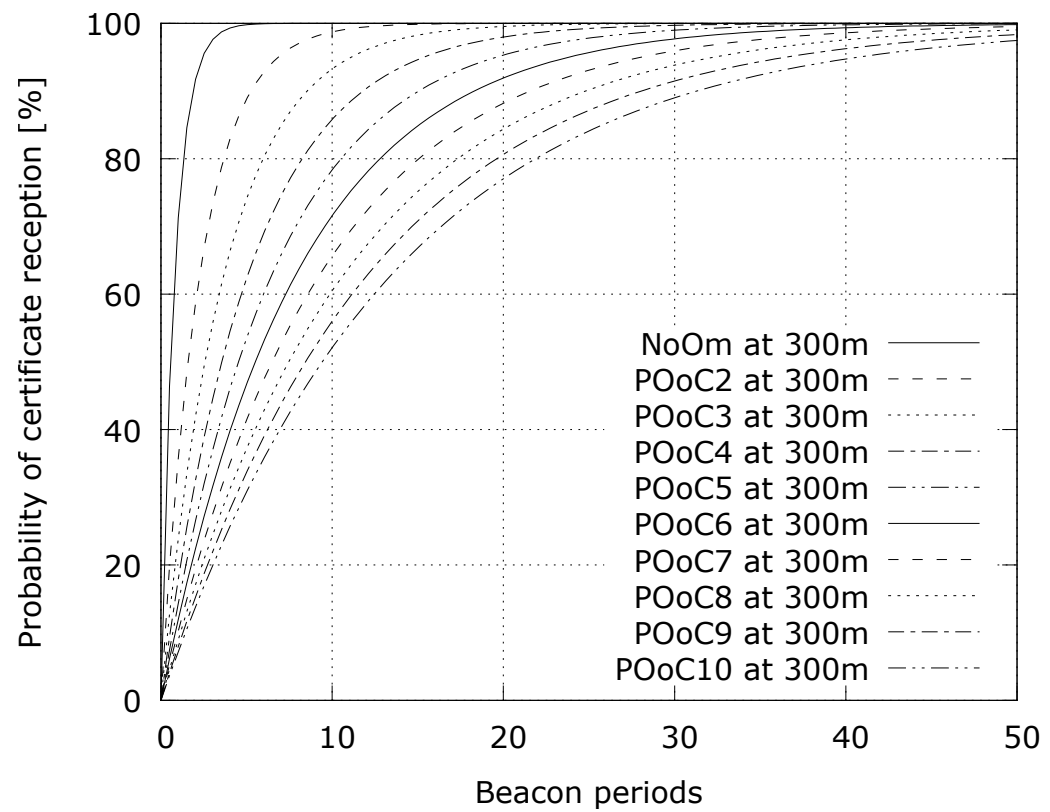

Figure 11.9: Probability of certificate reception after $n$ beacon periods without load in the communication channel at 3oom distance

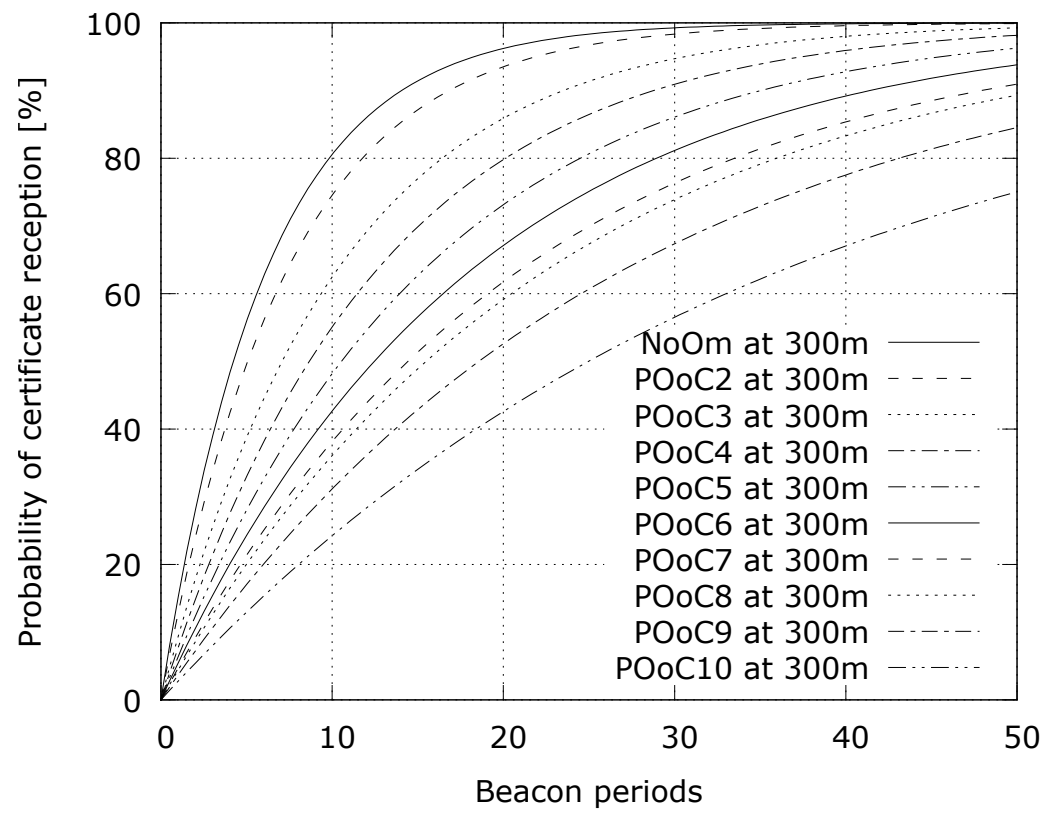

Figure 11.10: Probability of certificate reception after $\mathrm{n}$ beacon periods with high load in the communication channel at $300 \mathrm{~m}$ distance 


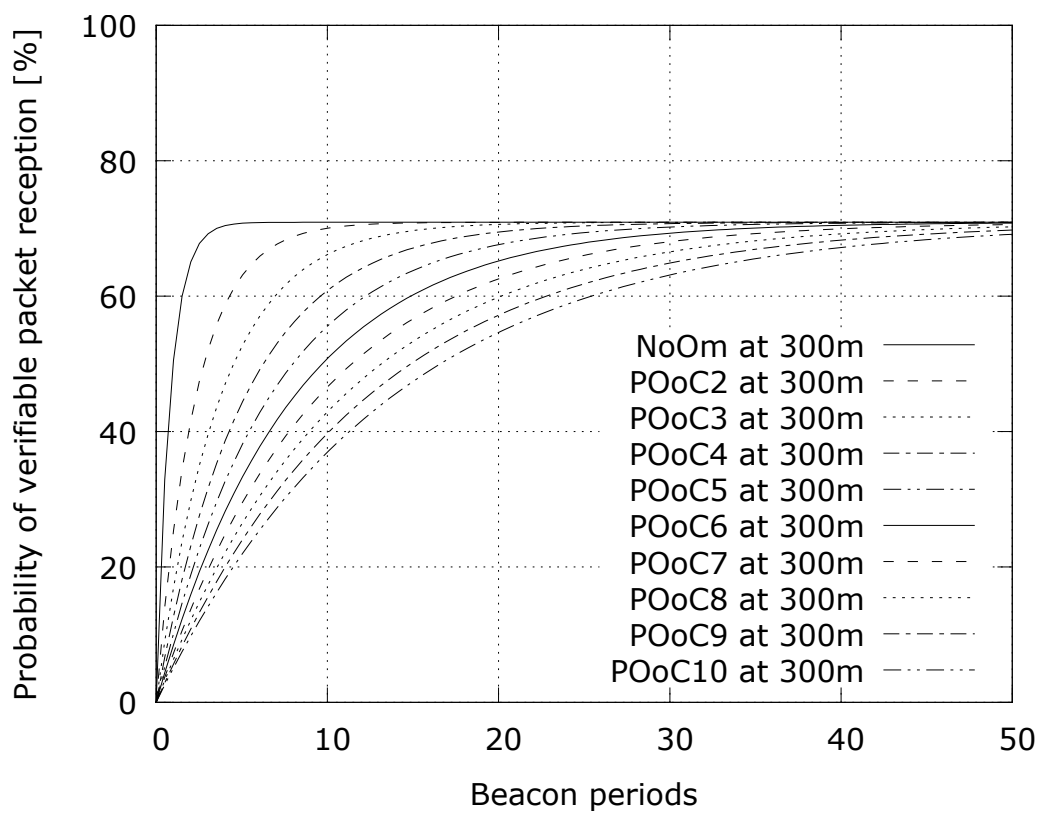

Figure 11.11: Overall packet delivery rate considering CPL and NPL without load in the communication channel at 30om distance

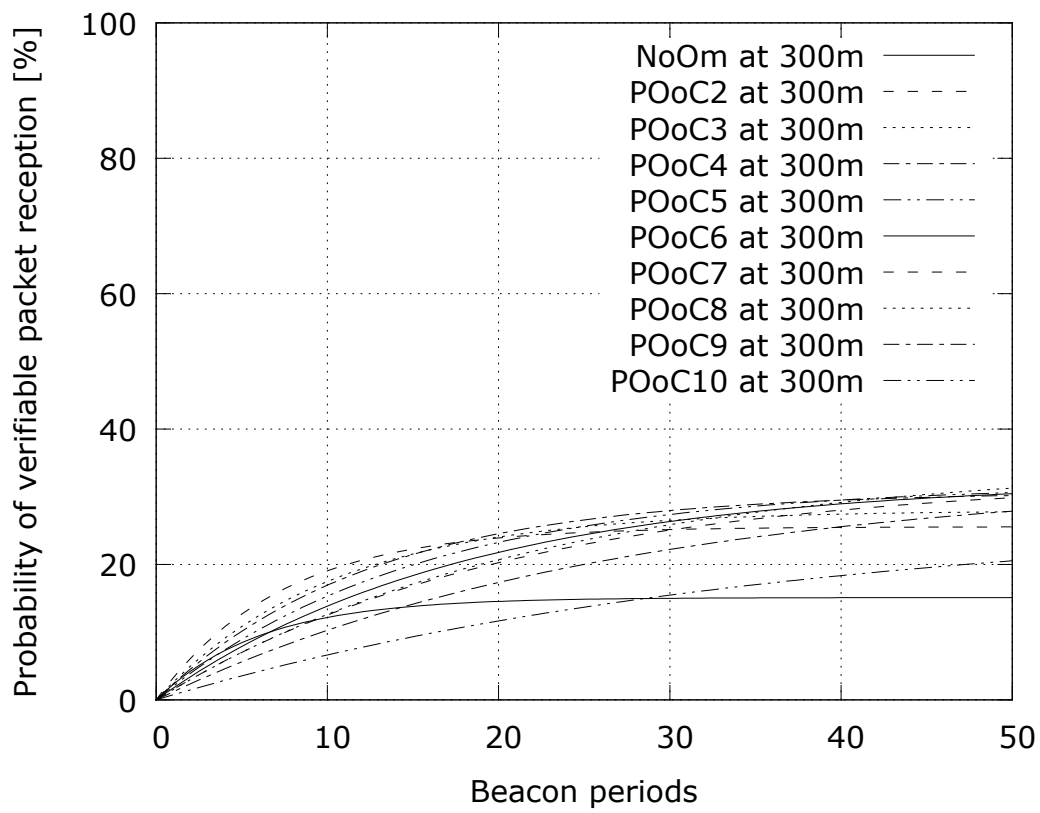

Figure 11.12: Overall packet delivery rate considering CPL and NPL with high load in the communication channel at $300 \mathrm{~m}$ distance 


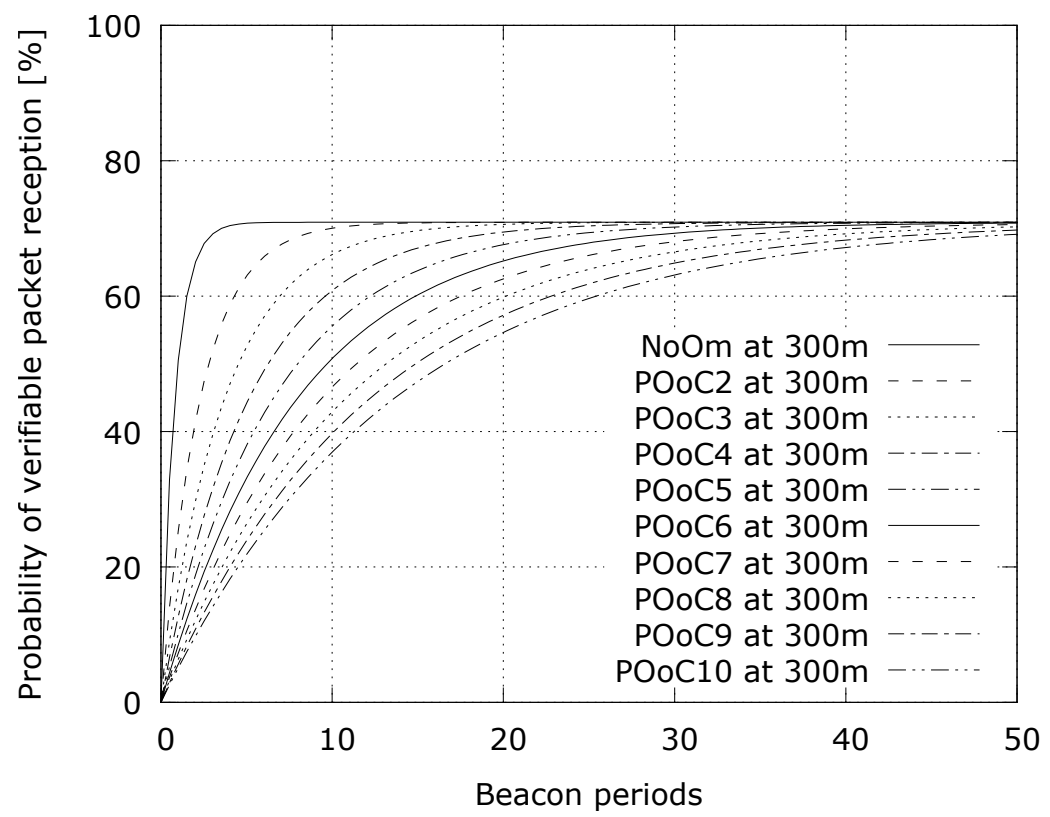

Figure 11.13: Overall packet delivery rate considering CPL and NPL with high load in the communication channel at $200 \mathrm{~m}$ distance

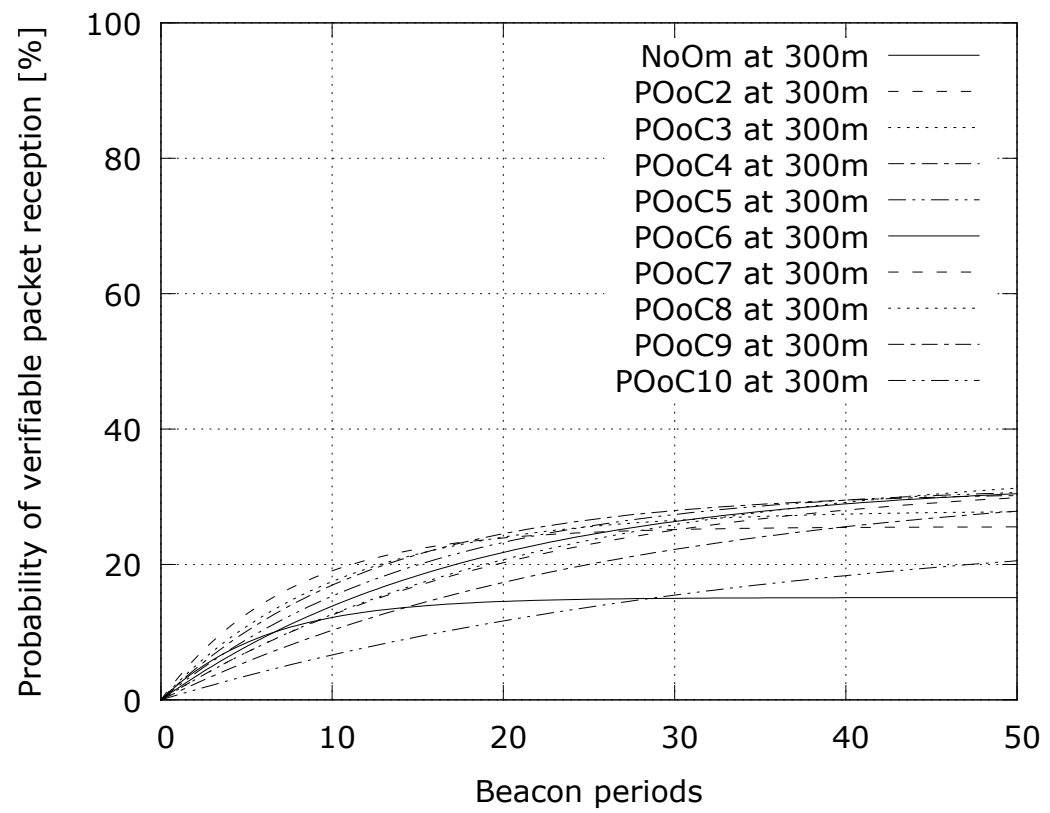

Figure 11.14: Overall packet delivery rate considering CPL and NPL with high load in the communication channel at 1oom distance 



\section{2}

\section{CONGESTION BASED CERTIFICATE OMISSION}

\subsection{CONGESTION-BASED CERTIFICATE OMISSION SCHEME}

The omission of certificates in authenticated one-hop broadcast messages is an effective way to reduce load on a communication channel. However, this improvement implies a trade-off against the immediate verifiability of messages. As we have discussed in Chapter 11, certificate omissions schemes can create situations where messages cannot be verified due to missing certificate material. An effect which we call cryptographic packet loss (CPL), as opposed to network packet loss (NPL), which can occur due to classic network effects such as collisions, buffer overruns, or packet expiration. A trade-off is necessary between communication load and cryptographic verifiability. In this chapter we discuss our proposal for a new omission scheme, which strikes a different balance between CPL and NPL. Our approach is based on the evidence from Chapter 11.3, which indicated that the existence of actual congestion is a key designator that certification omission can achieve any benefits at all. At the same time we want to ensure robust elimination of CPL, especially in situations where certificate omissions will be useful, i.e. in scenarios with high congestion. We present simulation studies, which are in line with previous evaluation efforts, to support our proposals.

Certificate omission schemes for vehicular communications have been proposed by Calandriello et al. [17], which are based on fixed periodic omission. This is an effective, predictable and robust scheme. But it is intuitively clear that a fixed scheme might not be the most efficient scheme possible. Schoch et al. [128] tried to optimize both the communication load and the cryptographic latency by proposing an event based scheme. The arrival of a new neighbor in communication range would trigger the inclusion of certificates. Otherwise the inclusion of certificates is omitted. However, this scheme is not robust in highly congested scenarios. Frequent arrivals of new neighbors in an already congested scenario lead to extra congestion in the channel, as certificate omission is effectively suppressed. Furthermore, limitations of cache management, such as storage space limitations or a high rate 
of packets loss that makes certificates appear stale, can lead to an unjustified purge of certificates from a local cache of certificates. This would again lead to the suppression of certificate omission because missing certificate material will be falsely interpreted as a neighborhood change event. This is suppression of certificate omissions is exactly opposite to the behavior we would expect to see. A scenario with very high levels of congestion calls for more aggressive omission in order to ensure availability of the communication channel. While the idea to use events for absolute optimal omission is intuitively valid, we note that in practice the behavior of an event based scheme can lead to suboptimal behavior. A scheme that is directly based on channel congestion and not on the mobility of other vehicles should provide better robustness.

We propose to optimize omission of certificates not towards the absolute maximal number of omissions, but towards optimizing the tradeoffs between communication load (NPL) and cryptographic packet loss (CPL). To achieve this, we consider the load on the communication channel as the guiding metric. If the communication channel is uncongested, then there is no need for trading in cryptographic packet loss for less load on the channel. And, if the communication channel is congested, we want to reduce the communication load by aggressively omitting certificates. While aggressive omission increases the cryptographic packet loss, it will decrease the overall NPL due to the reduced size of messages.

With Congestion-based Certificate Omission (CbCO)[47], we propose to optimize omission of certificates not towards maximizing the number of omissions but instead towards minimizing the overall packet loss and thus optimizing the trade-offs between communication load and CPL. To achieve this, $\mathrm{CbCO}$ considers the load of the communication channel as the guiding metric. If the communication channel is free, there is no need to trade in CPL for less load on the channel. And if the communication channel is congested we want to reduce the communication load by aggressively omitting certificates. While aggressive omission increases the CPL, our evaluation shows that it will likewise decrease the overall NPL due to the reduced size of messages at an even larger rate, yielding an overall positive effect on packet loss. Figure 12.1 shows an example of $\mathrm{CbCO}$ where a congestion is detected on the third beacon. Then, the beacons 4 through 6 are transmitted without certificate.

$\mathrm{CbCO}$ is based on POoC and omits certificates on a periodic schedule. However, the certificate rate $n$ at which certificates are added is flexible and adaptive, based on the locally measured congestion of the communication channel. Direct information about the congestion level in a wireless communication channel is accessible locally at the MAC layer of the communication stack. Accessing this information directly across layers can be impractical. However, a certificate management 


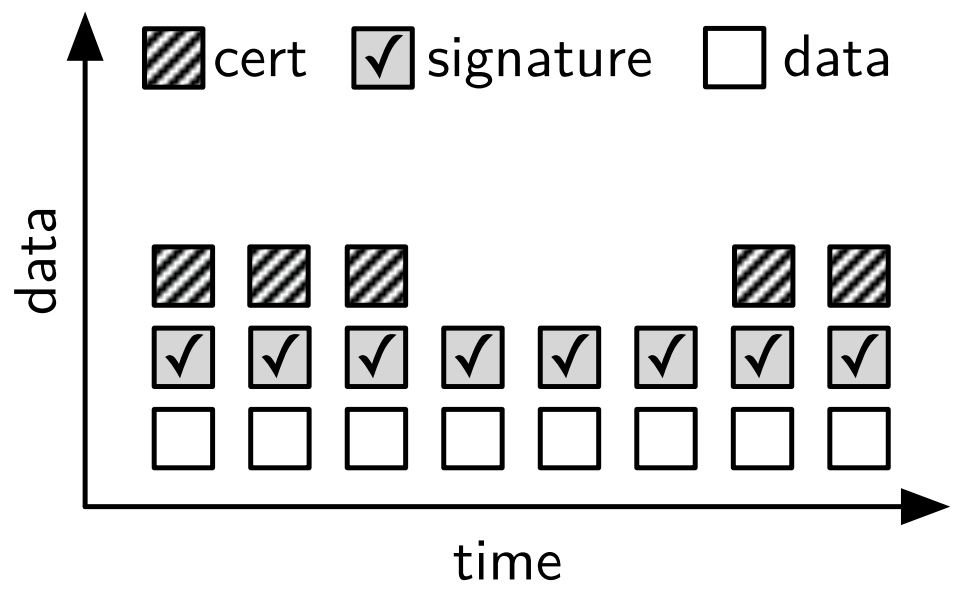

Figure 12.1: Example of $\mathrm{CbCO}$

subsystem will maintain a neighbor table with a cache of recently seen certificates. The number of vehicles in communication range can be derived from the size of this neighbor table or cache of recently seen certificates. The larger the size of the neighbor table, the larger we choose $n$. If $N$ is the total size of the neighbor table, then $n=\lfloor\Omega(N)\rfloor$, where $\Omega$ is a weight function. This weight function defines the maximum number of omission in function of the channel occupancy. As $\Omega$ is a key parameter of the $\mathrm{CbCO}$ scheme, we analyze three different trends to determine the optimal strategy. We consider $n_{\max }$ the size of the neighbor table that should trigger maximum omission and $\mathrm{o}_{\max }$ the maximum omission rate. The selection of appropriate values for $o_{\max }$ and $n_{\max }$ is discussed in Section 12.2.1. We evaluate the following functions for $\Omega$ :

$$
\begin{array}{r}
\Omega_{\text {linear }}: y=\min \left(\frac{x}{n_{\max }} \cdot o_{\max }, o_{\max }\right) \\
\Omega_{\text {quad }}: y=\min \left(\left(\frac{x}{n_{\max }}\right)^{2} \cdot o_{\max }, o_{\max }\right) \\
\Omega_{\text {trig }}: y= \begin{cases}-\cos \left(\frac{\pi}{n_{\max }} \cdot x\right) \cdot \frac{o_{\max }}{2}+\frac{o_{\max }}{2}, & x<n_{\max } \\
o_{\max }, & x \geqslant n_{\max }\end{cases}
\end{array}
$$

The core insight of using weight function to adjust a certificate omission period is essentially independent of the particular configuration of the weight function. Our selection of linear, quadratic, and trigonomet- 
ric functions is simply a representative selection of primitives function types. For our studies we select $o_{\max }=10$ to limit the worst case of $\mathrm{CbCO}$ to 10 omissions, which represents POoC-11. This setting is based on the general assumption that critical messages contest, such as CAM messages, should be broacast at least at a frequency of $1 \mathrm{~Hz}$. Setting POoC with a period of 10 as a baseline with a beaconing rate of $10 \mathrm{~Hz}$ reults in exactly this desired minimum broadcast frequency. The floor of $n=0$ represents no omissions of certificates, i.e. POoC- 1 or NoOm. We choose $n_{\max }=100$ as an approximation for a congested communication channel based on preliminary results from the PRESERVE project [133]. Figure 12.3 shows the resulting graphs of omission frequencies for the three $\Omega$ functions we consider for $\mathrm{CbCO}$ in our study.

\subsection{EVALUATION}

To evaluate the performance of $\mathrm{CbCO}$ omission schemes we focus on a simulation scenario with variable levels of congestion, which is controlled by the number of vehicles in the scenario. A simplified abstract formal model in Chapter 11.3 already indicated that the influence of certificate omission on overall packet delivery success will be highly dependent on congestion of the communication channel. Consequently it makes sense to investigate the effectiveness of $\mathrm{CbCO}$ omission with respect to performance under variable levels of congestion in the communication channel. More comparisons with other certificate omissions schemes and a different application level metric will be presented in chapter 13 .

We use a simulation package based on JiST/SWANS [4] with extensions by the University of Ulm. ${ }^{1}$. The simulation environment provides 802.11p radio simulation and a realistic vehicular mobility model called STRAW [26], which uses map data from the U.S. Census Bureau. This simulation package allows us to efficiently simulate scenarios with a high density of vehicles [129], which is our main interest for the evaluation of congestion-based certificate omission. We use a $3 \mathrm{~km}$ by $3 \mathrm{~km}$ urban city map in Suffolk County, U.S.A.. This scenario was previously used to evaluate the performance of Neighbor-based Certificate Omission $(\mathrm{NbCO})$ [128] and is selected here again to maintain comparability and because it is publicly available in the aforementioned extension package of the JiST/SWANS package by the University of Ulm.

For the same reasons, all other basic parameters for our simulation are also kept in line with previous works by Schoch et.al [128] This includes payload and certificate sizes, noise and channel propagation models, as well as vehicle placement and mobility. A summary of

1 Website: http://www.vanet.info 
Table 12.1: Cryptographic settings

\begin{tabular}{ll}
\hline Parameter & Value \\
\hline PKAlgorithm & nistp256 \\
ECC Key Type & compressed \\
Cert Size & 140 Bytes \\
Signature Size & 65 Bytes
\end{tabular}

the simulation parameters is given in Table 12.2. Chapter 11.3 feature discussions of the common settings that are applied to all simulations studies in the context of our simulation studies on certificate omission.

In our simulation we consider only the transfer of one-hop beacon messages in the single control channel (CCH) of IEEE 802.11p. While one-hop beacon messages will not be the only safety messages in the $\mathrm{CCH}$, we assume that these messages will dominate the load. The configuration of the 802.11p communication channel is set to $3 \mathrm{MBit} / \mathrm{s}$ with a fixed transmission power of $10.9 \mathrm{~dB}$ for robust delivery of one hop safety messages [91]. A summarized description of the cryptographic additions to our simulated messages is included in Table 12.1. Adding the 45 byte BSM and 5 byte for headers in the payload to the cryptographic material, the total size of one beacon message is 255 bytes with certificate and 115 bytes when omitting the certificate. Chapter 11.I includes further discussion of the parameters that are common for all simulation studies in this dissertation.

The beaconing rate in our simulations is fixed at $10 \mathrm{~Hz}$, as recommended by SAE J2735. A full simulation run simulates 60 seconds of traffic. The analytical model in chapter 11.3 and the investigations in chapter 13 show that this is enough time for the scenario to converge to a stable state. During the time of our simulations we do not simulate pseudonym changes. We expect the rate of pseudonym changes to be low enough to not be a relevant factor for the bandwidth optimization of beaconing services[108]. A discussion of the utility of certificate pre-distribution for the support of co-ordinated pseudonym changes is provided in the conclusions of chapter 14 .

To test the efficiency of omission schemes under high loads, we scale the number of vehicles in the simulation scenario between 100 and 1300 vehicles on a $3 \mathrm{~km} \times 3 \mathrm{~km}$ road network. On the selected map, this leads to an average of 5 to 68 vehicles in communication range and 18 to 252 vehicles in sensing range. Figure 12.2 show a graphical representation of these values, which were generated through a measurements within the simulation scenario. 
Table 12.2: Simulation parameters

\begin{tabular}{ll}
\hline Parameter & Value \\
\hline Field size & $3 \mathrm{~km} \times 3 \mathrm{~km}$ \\
MAC & $802.11 \mathrm{p}, 3 \mathrm{MBit} / \mathrm{s}$ \\
Fading & Rayleigh \\
Pathloss & Two-ray ground \\
Noise & Additive \\
Simulation time & $60 \mathrm{~s}$ \\
Simulation runs per configuration & 10 \\
Transmit power & $10.9 \mathrm{~dB}$ \\
Beaconing frequency & $10 \mathrm{~Hz}$ \\
Payload Size & $50 \mathrm{Bytes}$ \\
Number of nodes & $100, \ldots, 1300$
\end{tabular}

\subsubsection{Analysis}

For the analysis of our scheme we first investigate the settings for congestion-based certificate omission. The guiding metric we use as the foundation for congestion-based certificate omission is the number of neighbors in communication range. This metric and a basic model of a 802.11p CCH with a $10 \mathrm{~Hz}$ BSM application on top enable us to derive the congestion on the channel. In our simulations we identified an approximate limit of 1000 BSMs per second to saturate one communication channel in 802.11p wireless communications. We derive that 100 vehicles in communication range sending BSMs at $10 \mathrm{~Hz}$ represent a natural limit of the communication channel.

The authenticated delivery of BSMs is a cornerstone of various safety applications. To achieve a robust delivery of verifiable BSMs, it is reasonable to consider an upper bound on the maximum number of omissions our scheme allows. As a guideline we use a recommendation in Annex B2.2 of IEEE 1609.2 v2 D12 [63] and in Annex B of ETSI TS 102637-2 draft [39]. The IEEE 1609.2 recommends to include a full chain of certificates instead of just a single certificate at least once per second. In ETSI TS 102637-2 there is a description of Cooperative Awareness Messages (CAM), which are the equivalent to BSMs in the European standardization process. There, we find a set of informative rules for context adaptive beaconing rates, which specifies a maximum time between beacon generation of one second. From this, we deduce that 


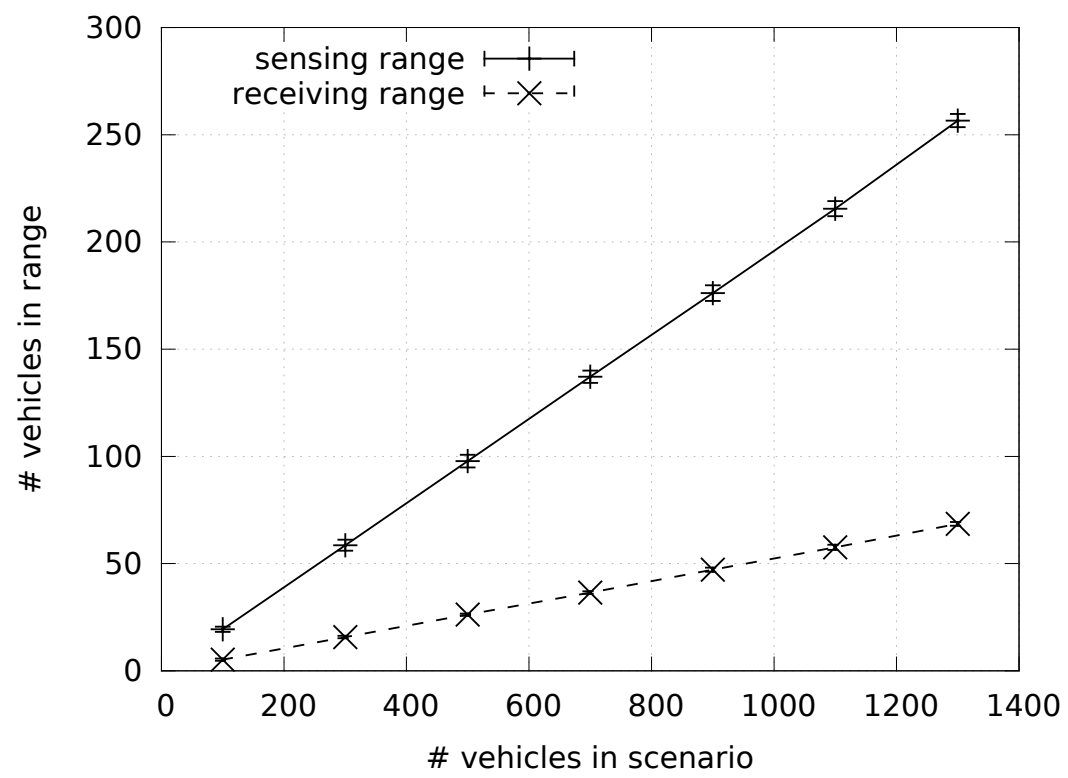

Figure 12.2: Connectivity measurements in the selected scenario

the same interval of one second between the inclusion of a full set of authentication material should be considered as a useful upper bound or $\mathrm{o}_{\max }=10$.

With the bound on the communication channel and the bound on the maximum number of omissions we have a framework to define specific values for our $\mathrm{CbCO}$ scheme. Figure 12.3 shows the resulting $\Omega$ functions for $o_{\max }=10$ and $n_{\max }=100$.

While the linear function serves as a baseline to scale the number of omissions directly related to the number of neighboring vehicles, the other functions further reduce the omission rate at lower vehicle densities to prevent CPL when there is no direct benefit in reduced NPL. We generally want to keep the number of omission low until the channel becomes congested and benefits from reducing NPL. For this reason we propose two additional ways to calculate the number of omissions. A quadratic function lets the number of omissions grow slower in less densely connected environments. And a trigonometric function produces similarly slow growth of omissions on sparsely connected environment while accelerating the increase of omissions more aggressively in densely populated environments.

To assess the quality of our $\mathrm{CbCO}$, we begin by analyzing the number of omissions with the different variations of $\mathrm{CbCO}$. Figure 12.4 shows the mean of the percentage of beacons sent with a certificate attached to it. This mean is the inverse of the average number of omissions. We 


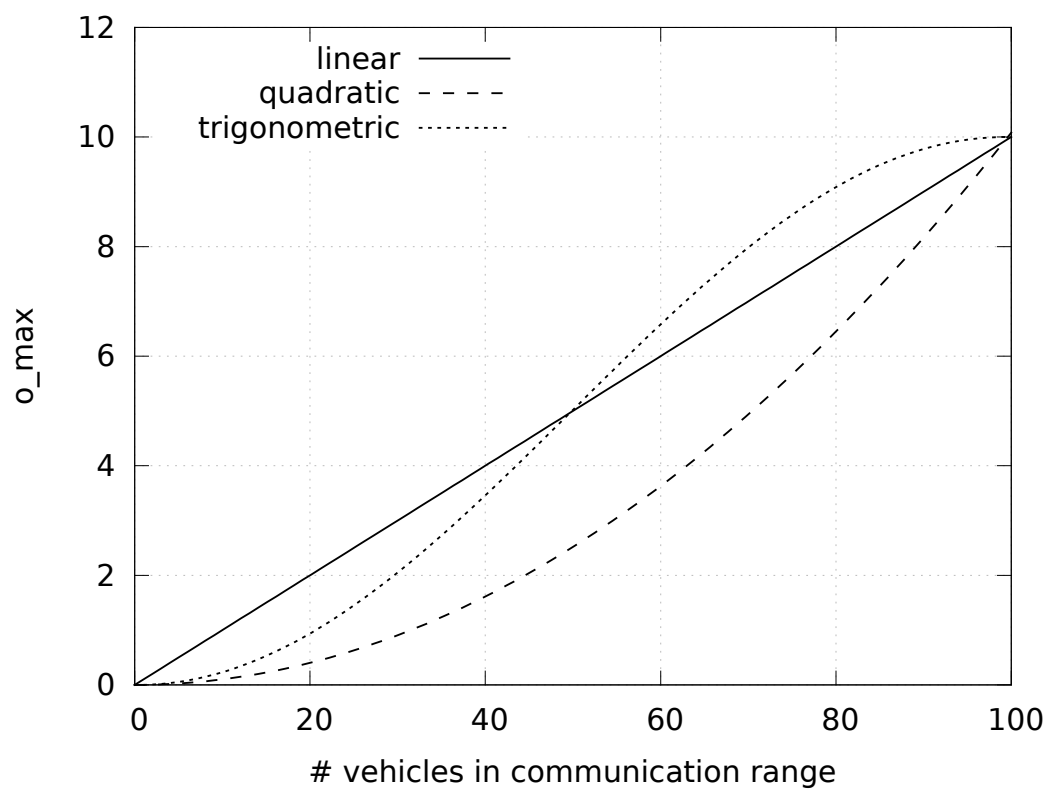

Figure 12.3: Omission rates strategies for congestion-based certificate omission

see the linear and trigonometric curves closely matching each other, while the quadratic calculation of omissions results in less omissions.

Next, we want to investigate the consequences of these different functions in terms of omissions. We measure this as cryptographic packet loss, i.e. the relative number of unverifiable messages that are dropped. This is shown in Figure 12.5. We see that again the linear and trigonometric approaches match quite closely, while the quadratic method results in fewer unverifiable messages. The slower increase in omissions with quadratic method clearly outperforms the other two variants in the avoidance of CPL.

The graphs of the means in Figure12.4 and Figure 12.5 do include deviation bars. However, the magnitude of the deviations is too small to make it noticeable. This is an indicator of relatively uniform connectivity rates, which is true especially as the number of vehicles increases. This is an artifact of the dimensions of the scenario and the constraint of vehicle placement along street grids. As the number of vehicles in the scenario grows, while the space for placement of vehicles in the scenario remains the same, the connectivity becomes uniformly high. This is the intended congestion of the scenario, which is required to show the different properties of the $\mathrm{CbCO}$ variations.

The effectiveness of avoiding CPL is only one aspect of the performance of a certificate omission scheme. The goal of certificate omissions 


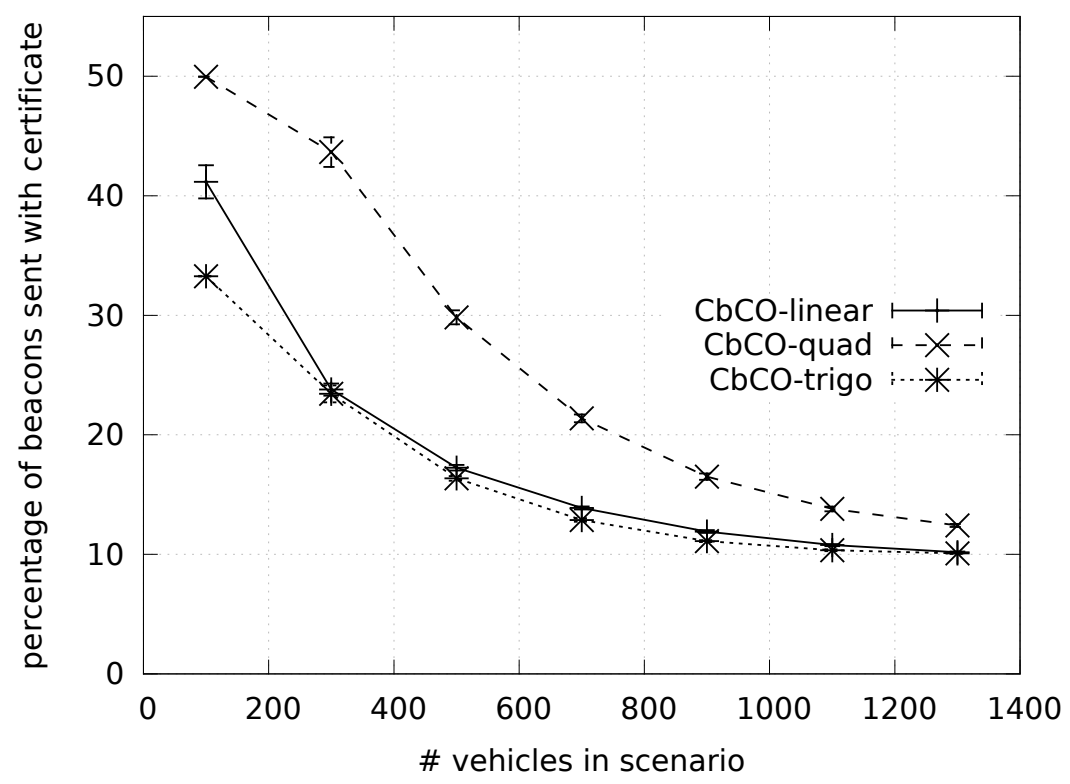

Figure 12.4: Average percentage of certificate omissions in $\mathrm{CbCO}$

is to enhance overall packet delivery by reducing the load on the network and thus fewer packet loss due to classic network effects (NPL). To see this effect we calculate a baseline of successful message delivery without any inclusion of any certificates. Using this baseline we can calculate the added packet loss due to the inclusion of certificates. Since different omissions schemes result in different numbers of omissions we see different characteristics for each scheme.

The graph in Figure 12.6 shows the average increase in network packet loss (NPL) relative to packets without certificates. As a reference, we also show the additional packet loss for no omissions (NoOm). As one can see, $\mathrm{CbCO}$ achieves a significantly reduced packet loss due to reduced message size compared to the NoOm scheme. One can also see a small gap between the quadratic $\Omega$ function and the other two variations of the $\Omega$ function. This gap directly corresponds to the differences in average certificate omissions, which was shown in Figure 12.4. This is correlation is clear, as this figure only shows the effect of pure NPL effects.

Our goal is to decrease overall packet loss, under consideration of NPL and CPL. This is shown in Figure 12.7. First of all, we can again observe the benefits of the omission schemes compared to attaching certificates to all packets. There is also a slight advantage of the linear and trigonometric $\Omega$ functions. Additionally, we note that above 1000 vehicles, we see the effect of the bounding of omissions, as the differ- 


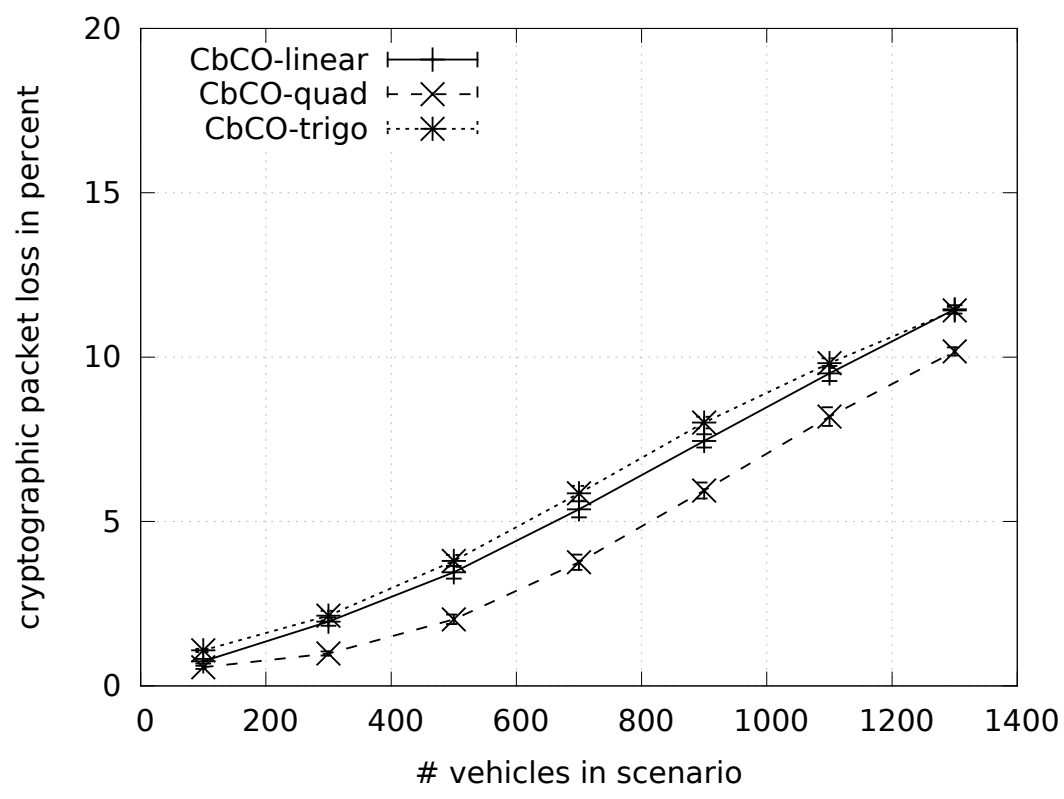

Figure 12.5: Average percent of unverifiable messages among received messages

ent $\Omega$ functions converge. Figure 12.8 illustrates the composition of network packet loss and cryptographic packet loss using $\mathrm{CbCO}$-linear as an example.

While $\mathrm{CbCO}$ introduces additional CPL, it is evident from Figure 12.7 that the positive effect of reduced NPL outweighs this negative effect by far. However, we still need to investigate whether this comes at the expense of increased latency until a communication partner receives the certificate required to start authenticating messages.

Figures 12.9 and 12.10 illustrate the maximum and average number of unverifiable beacons until arrival of the certificate. As for the number of omissions, we notice that the quadratic method has a lower latency until messages become verifiable. We note that the linear way to calculate the congestion-based omission seems to provide a slightly improved latency characteristic compared to the trigonometric function.

We conclude that the linear and trigonometric approaches perform very similar, with slight advantages for the linear approach. We consider the advantage of the linear approach to be rooted in the faster increase of omissions in situations with high channel load. These situations generally have more impact on the simulation results and keeping the number of omissions down until the channel is overloaded is an effective approach. The quadratic function shows the limit of following 


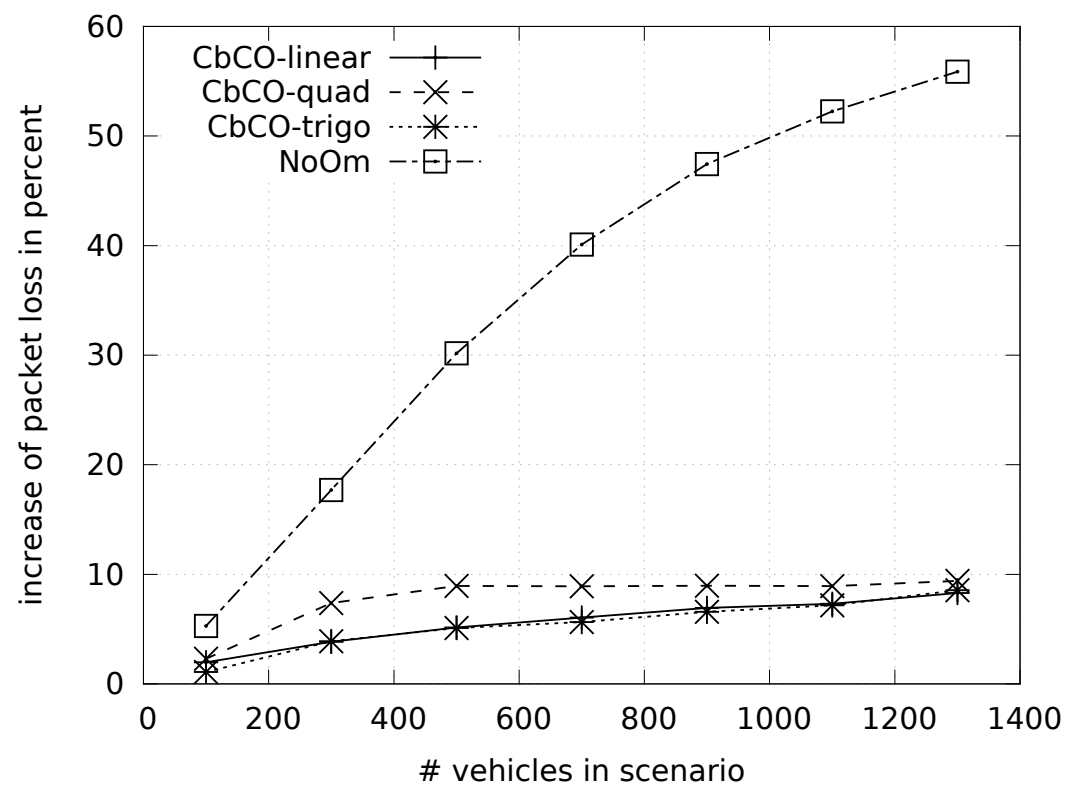

Figure 12.6: Increase of packet loss due to inclusion of certificates for different variants of $\mathrm{CbCO}$ (NPL only)

this line of thought. We see the advantage for the quadratic approach in the latency until a message becomes verifiable. Finally, we remark that the quadratic scheme showed a slightly worse overall increase of packet loss compared to the other approaches.

\subsubsection{Comparison with other Omission schemes}

To asses the utility of congestion based certificate omission we conduct comparisons to the following previously proposed omissions schemes:

- No omission of certificates (NoOm): This scheme serves as a baseline as it performs no omission.

- Periodic omission of certificates (POoC) [18]: The idea of the $\mathrm{POoC}$ is to add the certificate every $\mathrm{n}$ beacons. ${ }^{2}$ We evaluate two certificate periods of 3 beacons and 10 beacons.

- Neighbor-based certificate omission (NbCO) [128]: This scheme considers the context of a vehicle in the omission decision. The idea of $\mathrm{NbCO}$ is to only attach the certificate to beacons if there is a change in the neighbor table.

2 called certificate period in the original paper 


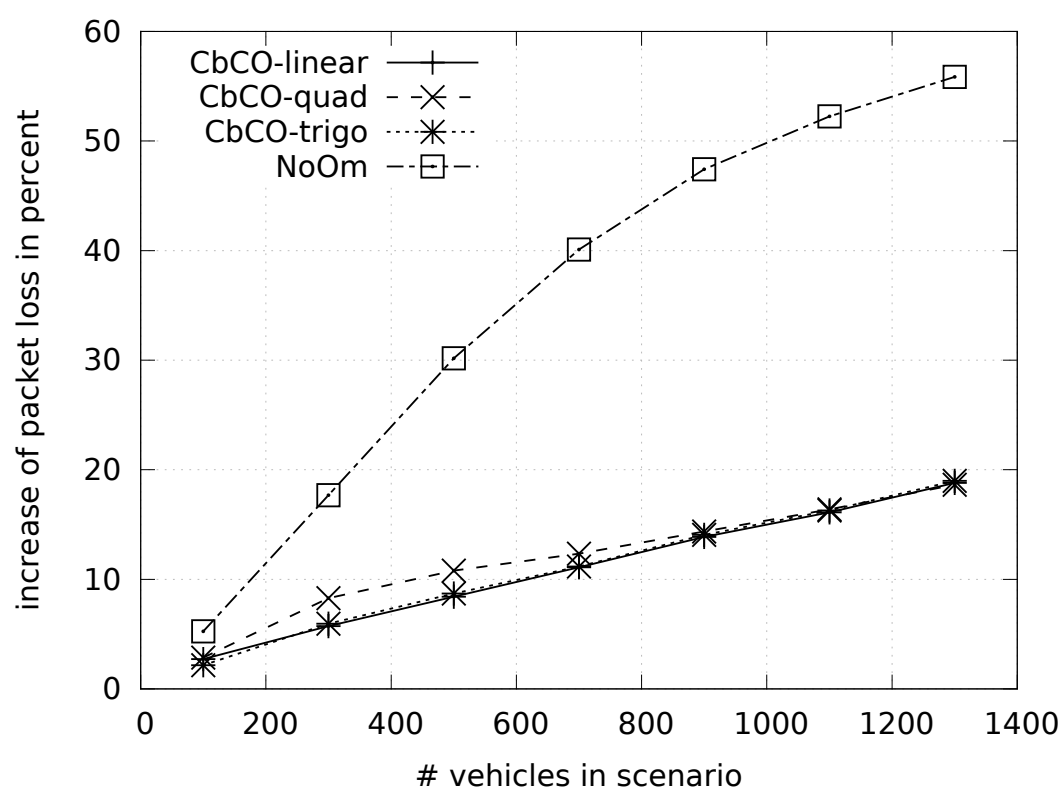

Figure 12.7: Increase of packet loss due to inclusion of certificates for different variants of $\mathrm{CbCO}$, counting NPL $+\mathrm{CPL}$

- Congestion-based certificate omission (CbCO) [48]: This scheme considers the load of the communication channel as the guiding metric. If the communication channel is free, there is no need to omit certificates to reduce the load on the channel. And if the communication channel is congested, then the communication load is reduced by aggressively omitting certificates. We evaluate two functions, which are used to adapt the omission frequency based on an implicit channel model and the neighbor table of vehicles: quadratic and linear.

The basic percentage of certificates included in messages is an indicator of the performance of each scheme. In Figure 12.12 we remark that the congestion based omission scheme is converging to the same $90 \%$ omission rate as the $\mathrm{POcC}-10$ scheme. On the other hand, the neighbor-based certificate omission scheme reduces omissions in densely populated scenarios due to the increased amount of neighbor changes in the network. This of course helps to keep down the CPL for the $\mathrm{NbCO}$ scheme, as can be seen as the mean value of unverifiable packets in Figure 12.13. The mean number of received unverifiable packets before the reception of a certificate is shown in Figure 12.11, which is an extension of the earlier Figure 12.10. 


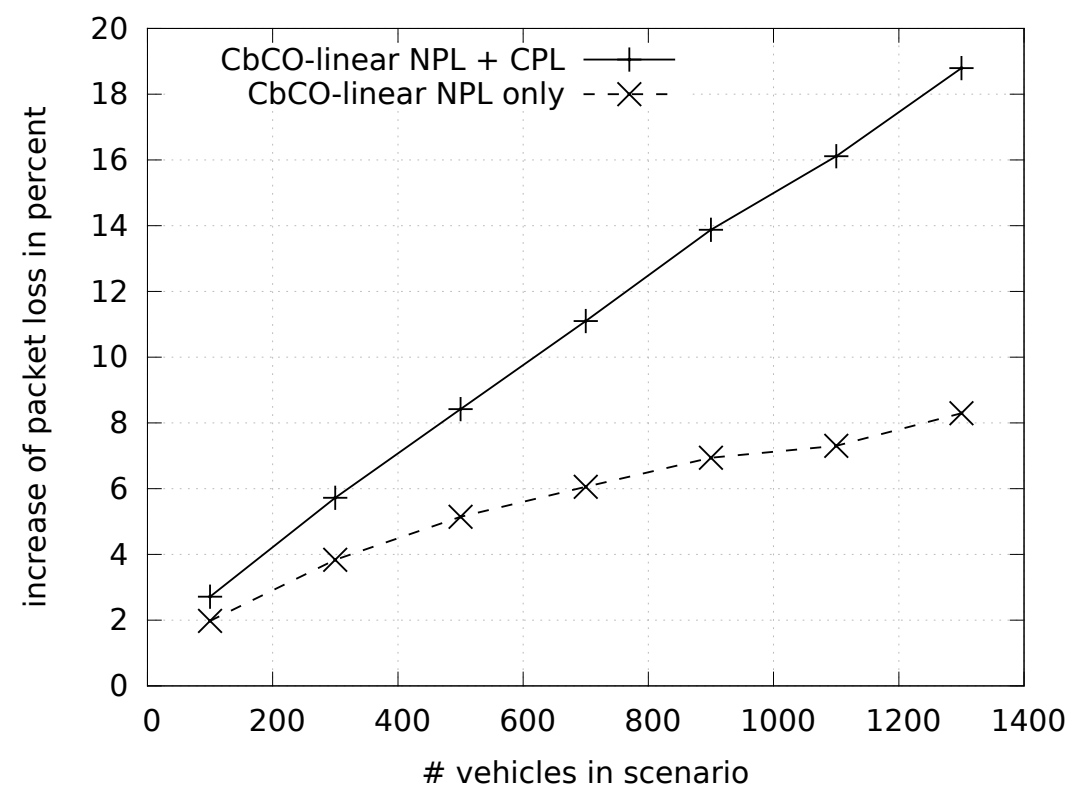

Figure 12.8: Illustration of the effect of combining cryptographic packet loss with regular network packet loss

The price for this low amount of CPL with the NbCO scheme is a much higher amount of regular NPL due to collisions in the communication channel as can be seen in Figure 12.14. All values are again relative to a baseline where we do not attach certificates at all (for NPL) or where every packet is assumed to be verifiable (for CPL). We note that the POoC scheme on the other hand performs well in terms of minimizing network packet loss but shows problems with regard to cryptographic packet loss.

Figure 12.15 finally presents an amortized total results for all types of packet loss induced by certificate inclusion. In this graph we consider unverifiable packets to be cryptographic packet loss and see that the congestion based omission schemes deliver the lowest amounts of packet loss, and thus best scalability in this overall view on the communication performance. Lastly, as a confirmation, we show in Figure 12.16 an extension of Figure 12.15 that includes the NoOm scheme as a baseline. The use of any of the investigated certificate omissions schemes is preferable to not applying any omissions at all. 


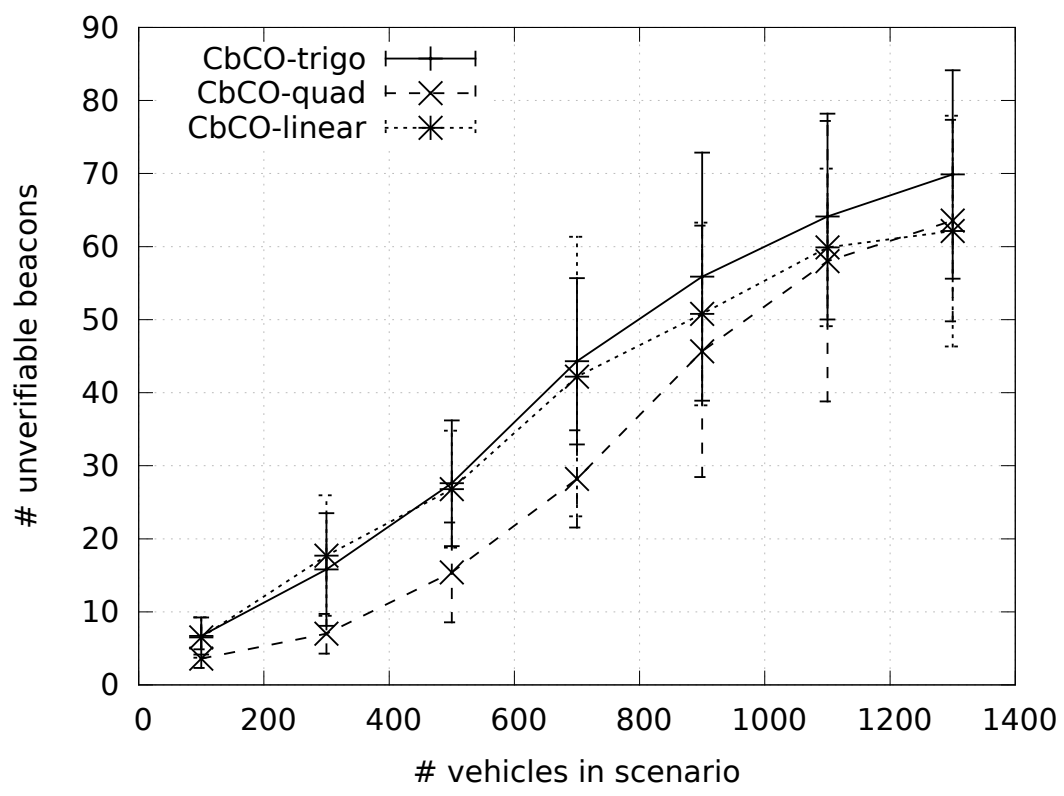

Figure 12.9: Maximum number of unverifiable beacons until arrival of certificate

\subsubsection{Discussion}

Our simulation study shows that the Congestion-based Certificate Omission scheme offers a balance between minimizing cryptographic packet loss and and achieving reduced network packet loss. The approach successfully keeps the amount of unverifiable packets low during times of low congestion, as shown in (cf. Figure 12.13, which is useful since there is not much benefit to be gained from aggressive omission at this stage. However, when it is beneficial to apply certificate omission, due to congestion in a communication channel, it can achieve high reductions of NPL, as seen in Figure 12.14. The combination of these two attributes leads to an overall improvement of packet delivery success under consideration of NPL and CPL effects, as illustrated in the Figure 12.15.

Using an adaptive mechanism to adjust the omission of certificates can reduce NPL as effectively as the most aggressive POoC non-adaptive POoC schemes, while providing better characteristics to reduce cryptographic latency and cryptographic packet loss. This way the $\mathrm{CbCO}$ scheme can context sensitively provide the best attributes of lower and high settings of $\mathrm{POoC}$.

Compared to the $\mathrm{NbCO}, \mathrm{CbCO}$ offers clearly superior overall scalability in highly congested scenarios, while essentially eliminating 


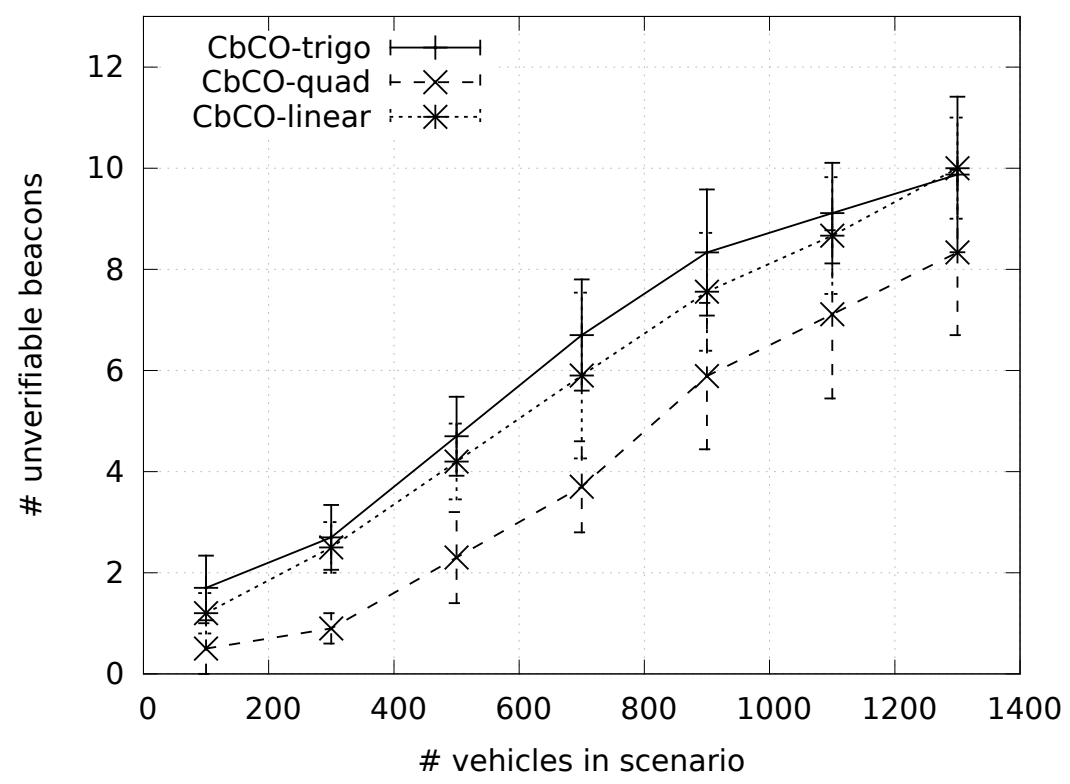

Figure 12.10: Average number of unverifiable beacons until arrival of certificate

the risk of CPL in scenarios without congestion. The event based approach to maximize certificate omission while optimizing cryptographic packet loss exhibited problems scaling to very high density scenarios. The frequency of certificate omissions in the $\mathrm{NbCO}$ omission scheme decreased with a higher amount of neighbor changes events in a congested scenario, as seen in Figure 12.12. This suppressed NPL savings at a time when saving bandwidth would have been most beneficial. While the $\mathrm{NbCO}$ is largely unaffected by cryptographic packet loss in Figure 12.10, we note that this figure only shows the average waiting time until reception of a certificate for successfully received beacons. Significantly more beacons are lost in the $\mathrm{NbCO}$ approach due to packet collisions in the communication channel. We conclude that the overall performance of $\mathrm{CbCO}$ provides a better balance between network scalability and avoidance of cryptographic packet loss.

While we did not consider pseudonym changes in this study we want to highlight that our scheme is more resilient against pseudonym changes in the network neighborhood than $\mathrm{NbCO}$. This is due to the fact that we do not consider the identity of neighbors as a factor but instead rely on only the actual congestion of the communication. In our simulation study this is derived from the number of neighbor, but in a real deployment this can be measured locally. Even a DoS attack by an 


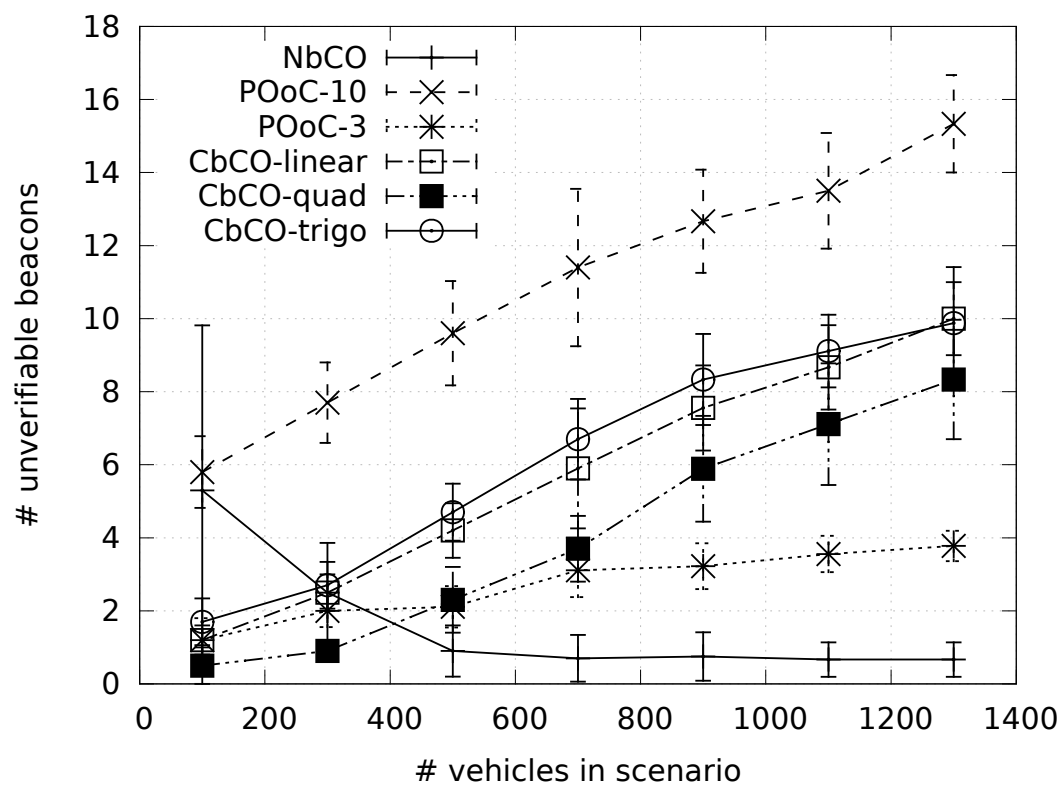

Figure 12.11: Average number of unverifiable beacons until arrival of certificate

adversary who sends a large number of beacons with changed sender addresses will only be measured as congestion and appropriately lead to more omission. This is the most useful behavior in a congested channel.

Overall, the simulation study in this chapter demonstrates that secure communication systems can and should channel state to make better decisions about security trade-offs. Measurements of mean packet delivery success under consideration of NPL and CPL showed that adaptive adjustment of omission frequency can reduce NPL when it is beneficial and avoid CPL when omission is not effective anyway. However, these measurements only provide a summary of events during the duration of a simulation run. The effects of CPL are only short lived, as seen in the graphs that show the latency times until verifiability is established. But for the situational awareness of vehicles in moving traffic, CPL occurs at a critical time. The first encounter of a new neighboring vehicles is an important event. Important enough for the $\mathrm{NbCO}$ scheme to optimize omission exclusively based on this event. A different metric is required to more precisely analyze the performance of omissions schemes with respect to CPL during the first encounter of new vehicles. For this purpose we will use a recent application layer metric for $\mathrm{V}_{2} \mathrm{~V}$ awareness as a finer grained metric in the next chapter. 


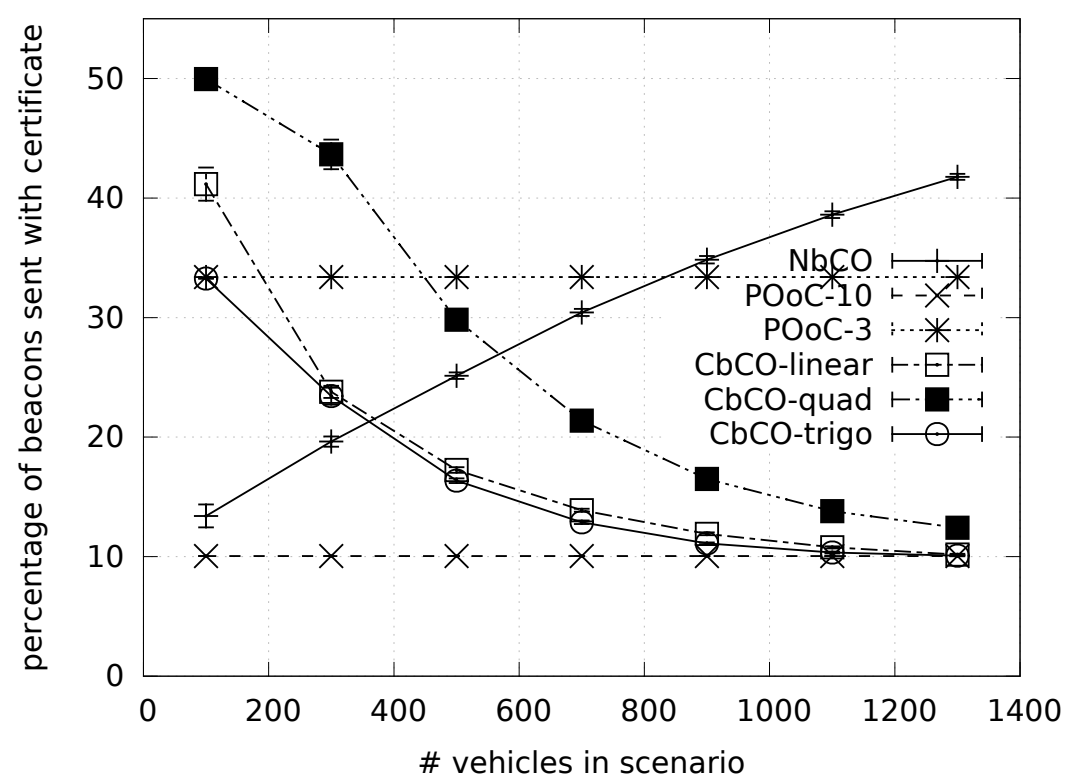

Figure 12.12: Average percentage of certificate omission in other protocols

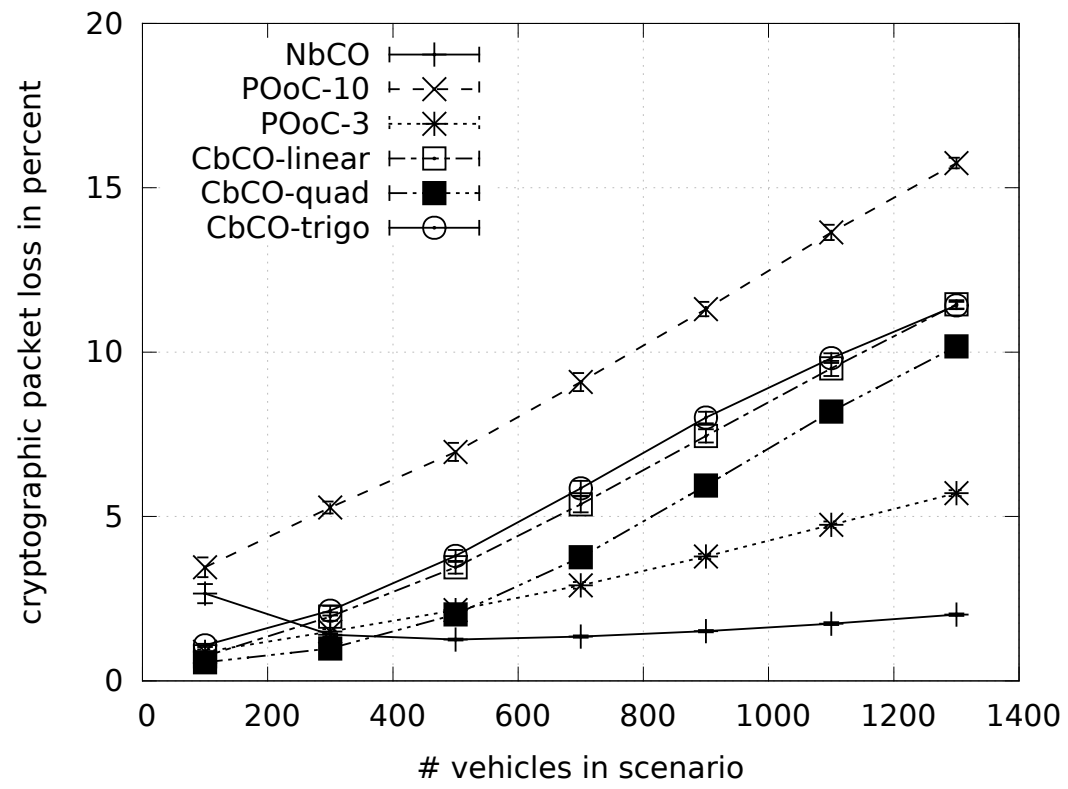

Figure 12.13: Average percent of unverifiable packets for various proposed omission schemes 


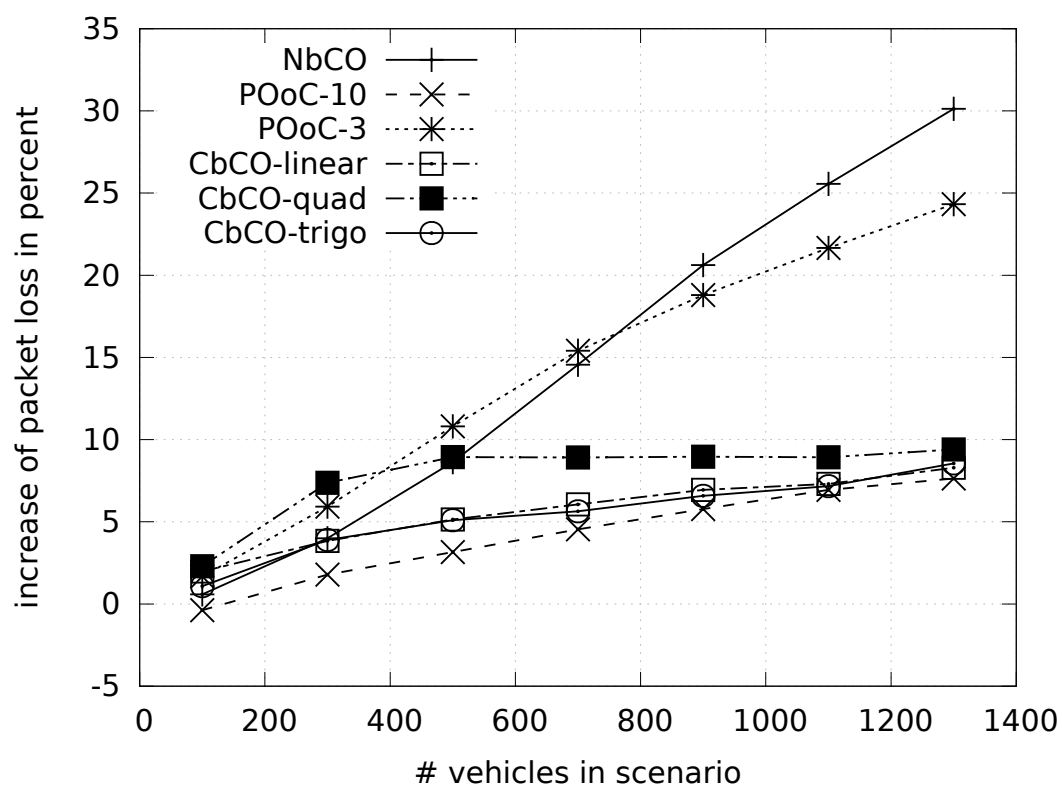

Figure 12.14: Increase of packet loss due to inclusion of certificates for different omission schemes (NPL only)

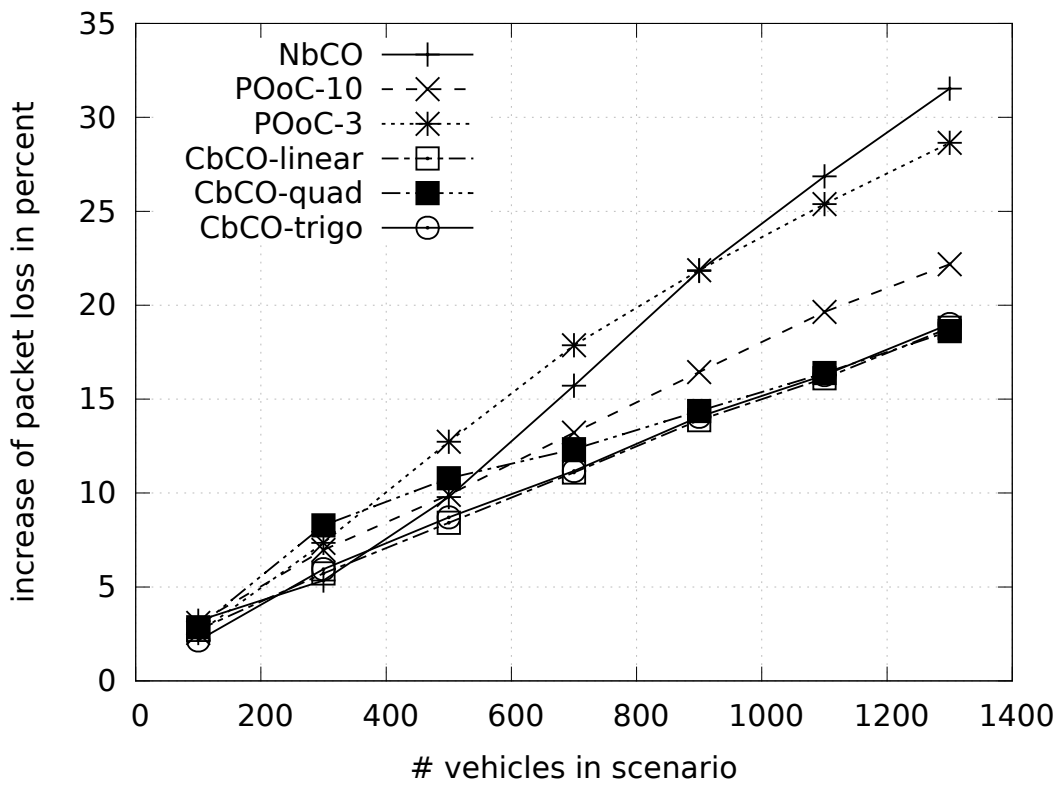

Figure 12.15: Increase of packet loss due to inclusion of certificates for different omission schemes, counting NPL + CPL 


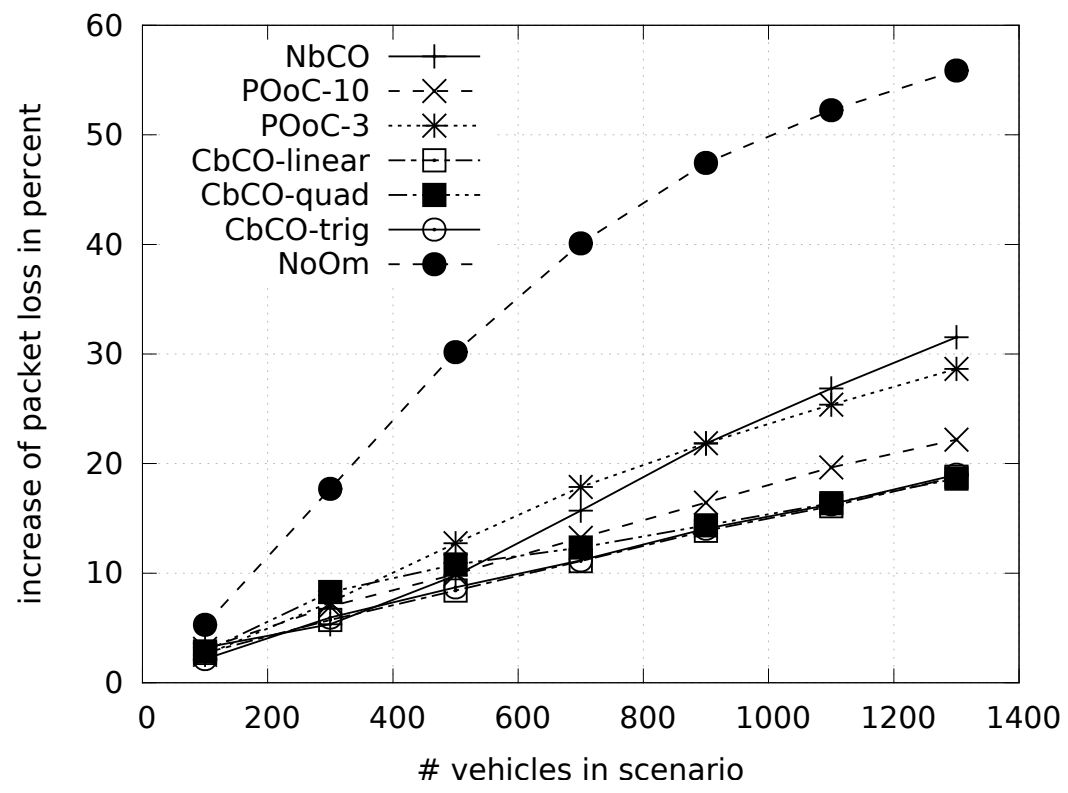

Figure 12.16: Increase of packet loss due to inclusion of certificates for different omission schemes, counting NPL + CPL. NoOm serves as a reference for no omissions 



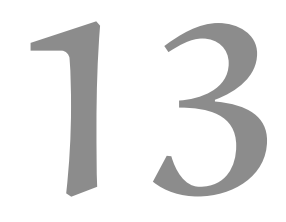

\section{APPLICATION LEVEL EVALUATION}

In the previous chapter 12 we evaluated a novel congestion based certificate omission scheme, based on mean packet loss statistic in a simulation study. This approach was also used in previous evaluations of certificate omission, such as [18, 48, 128]. We extended the existing approach by identifying NPL and CPL as distinct types of packet loss and evaluating them separately and combined. However, the observed effects always remain packet level summaries of the events in the simulation. This does not capture the impact of certificate omission on application level performance and it does not allow a detailed analysis of short lived aspects. We are particularly interested in analyzing the influence of certificate omission on the combination of CPL and NPL. Balancing the effects of bandwidth savings through NPL reduction with avoidance of unverifiable packets and CPL is a key performance attribute of certificate omission. To investigate this, we require a different metric that allows us to analyze the effectiveness of message and certificate delivery with a high degree of temporal resolution.

The only alternative metric that has been used in related works is the influence of certificate omission on an abstract emergency braking application. The authors of $[19,99]$ extrapolate from periodic certificate omission statistics to a number of car accidents, which their omission schemes can help to prevent. Their method is based on an assumption of an expected reduction of braking distance, which is also used in [107] to analyze the impact of authentication on an intersection collision avoidance system. An investigation of an emergency braking application is useful and appropriate, but it is not a universally applicable aspect. A more fundamental attribute that influences the service quality of safety applications is accurate knowledge of a vehicle's surroundings, which enables the creation of abstract situational awareness. For the investigations in this chapter we switch from measurements of mean packet loss statistics to the measurement of Awareness Quality. We use a definition of Awareness Quality proposed by Schmidt et al. in [124], where it was used as a metric to evaluate adaptive adjustments of Distributed Congestion Control (DCC) parameters [92]. 


\subsection{AWARENESS QUALITY}

Awareness Quality as an assessment of vehicular communication effectivity was first introduced as a metric in [124]. Awareness in this context is defined as the knowledge about the presence of neighboring vehicles. This knowledge is the main enabler for a multitude of cooperative driving services, such as lane changing, highway merging, intersection collision avoidance, platooning, and several others [6o]. The concept of awareness of surrounding vehicles is so fundamental for cooperative driving, that the basic position beacons, which create this information in the context of $\mathrm{V}_{2} \mathrm{~V}$ communication, are referred to as Cooperative Awareness Messages (CAM) in the ETSI family of vehicular communication standards.

The key insight to the usage of Awareness as a quantifiable metric is to compare the number of known vehicles to the number of vehicles that should be known. The need to calculate the number of vehicles that should be known required the presence of an all-seeing observer to collect this sort of ground truth. This implies that a vehicle cannot calculate its own Awareness Quality locally. However, an all-seeing observer can easily be implemented in simulation or in controlled test environments. The actual knowledge of a vehicle about a neighboring vehicle in this context refers to the recent reception of a position beacon from that vehicle. The validity time of a neighboring vehicles position beacon (also called freshness or beacon lifetime) is limited and may depend on the scenario. For static beacon periods it is possible to assume a static validity period for position beacons of neighboring vehicles. If beaconing frequencies are adapted dynamically, which is defined as an optional feature for CAMs in the ETSI framework citeETSI-TS-102637-2, the validity period needs to take this into consideration. The more common assumption for position beacons in vehicular communication is a static $10 \mathrm{~Hz}$ broadcasting frequency, which we follow as part of our previously discussed scenario assumptions in Chapter II. It is also possible to include tolerance for packet loss into the calculation of Awareness Quality by adjusting the beacon validity time. In [126] this is permitted based on the distance between sender of receiver of position beacons. This can be useful if tracking far away vehicles is less relevant for safety application. However, for the investigations in this chapter, we to stick to fixed validity times to achieve greater accuracy of our measurements.

Instead, we exploit another aspect of Awareness Quality to control for the the effects of distance dependence. In the informal introduction above we defined Awareness Quality as a relationship between the number of known vehicles compared to the number of vehicles that should be known. The definition of the set of vehicles that should be known can be adjusted to only consider subsets of neighboring vehicles. 
The natural way to define such a subset is as a circular area around a vehicle with a radius that is lower than the maximum possible area of communication. Or, to make measurements even more selective, we can consider ring shaped cut-outs from this area. This enables fine grained investigations of communication effectiveness with relevant neighbors. For example Awareness Quality of vehicles in very close proximity should be near 100\%, while a lower Awareness Quality can be more acceptable for larger vehicles at a medium or large distance.

Formally, we closely follow the original definitions in [125] for the notation of Awareness Quality. First, we need a definition of neighborhood area as subsets of the area around a vehicle. The original proposal of Awareness Quality refers to rings as safety areas $A_{k}$

$$
A_{k}=\pi *\left(a_{k}^{2}-a_{k-1}^{2}\right), k \in \mathbb{N}
$$

with $k$ denoting the identifier of the ring shaped areas, which are assumed to be concentric around the reference vehicle with

$$
a_{k}=a_{1} * k, k \in \mathbb{N}
$$

as the radius intervals, which grow in intervals of $100 \mathrm{~m}$, starting at $a_{1}$ being $100 \mathrm{~m}$. An alternative definition of these areas as circles instead of rings would work equivalently with

$$
A_{\mathrm{k}}=\pi *\left(\mathrm{a}_{\mathrm{k}}^{2}\right), \mathrm{k} \in \mathbb{N}
$$

Again following the definitions in [125], at time $\mathrm{T}$ and for a given vehicle $i$, its awareness about area $k$ is defined as

$$
\operatorname{Awareness}_{k}^{\top}(i)=\frac{\left|\mathcal{N}_{k}^{\top}(i) \cap \mathcal{V}_{k}^{\top}(i)\right|}{\left|\mathcal{V}_{k}^{\top}(i)\right|}
$$

with $\mathcal{V}_{k}^{\top}(i)$ denoting the set of all vehicles within area $k$ (i.e. the ground truth) and $\mathcal{N}_{k}^{\top}(i)$ denoting the set of known neighbors within area $k$. The intersection $\mathcal{N}_{k}^{\top}(i) \cap \mathcal{V}_{k}^{\top}(i)$ formally ensures that only those vehicles are considered, that are truly part of the set of vehicles in the relevant target area at exactly time T. Known neighbors in this context refers to all neighboring vehicles have that have successfully transmitted a position beacon within the time limits of a beacon lifetime definition. Vehicles that just enter or leave the neighborhood area are considered to be know during the full period of beacon lifetime. 
Since Awareness Quality is tied to exactly one vehicle $i$ at one specific point in time $\mathrm{T}$, we finally allow Awareness Quality to be averaged over all investigated vehicles and denote this as

$$
\operatorname{AQL}(T, k)=\frac{\sum_{j=1}^{T} \sum_{i \in \mathcal{V}} \operatorname{Awareness}_{k}^{\top}(i)}{|T| \times|\mathcal{V}|}
$$

which shows the overall Awareness Quality in a complete scenario as a function of time $\mathrm{T}$ in the area $\mathrm{k}$.

The Awareness Quality metrics described above were introduced and first defined by Schmidt et al. in [124]. A functionally equivalent metric has been introduced independently in [14] under the name Neighborhood Awareness Ratio. Here, the cardinality of the set of known vehicles around a vehicle $i$ is defined as $N D_{i, r, t}$ under the assumption of a circular neighborhood area with radius $r$ and a beacon lifetime of $t$. This value is divided by the total number of actual neighboring vehicles under the same assumptions $\mathrm{NT}_{i, r, t}$, i.e.:

$$
N A R_{i, r, t}=\frac{\left|N D_{i, r, t}\right|}{\left|N T_{i, r, t}\right|}
$$

This metric is by definition limited to circular areas and does not allow investigation of subsets such as rings. The definition of the time component allows for a more fuzzy collection of measurement with a suggested granularity of 1 second intervals. The measurement in [14] have been collected to analyze the efficacy of cooperative awareness in a large European field operational test project.

\subsection{AWARENESS QUALITY UNDER CERTIFICATE OMISSION}

We use of Awareness Quality as a fine grained application level metric to asses the same certificate omission schemes as in the previous chapter. The only omission is the trigonometric variant of the $\mathrm{CbCO}$ scheme, which was found to behave essentially identical to the $\mathrm{CbCO}$ scheme with a linear adaptation function. An overview of these schemes is given in Table 13.1.

We generally use the same overall simulation calibration settings as described previously in Chapter 11.3. In this scenario beacons are sent every 100 milliseconds, as suggested by SAE J2735 [119]. The lifetime of beacon messages in the neighbor table of vehicles is fixed at 1.5 times the beacon interval, i.e. 150 milliseconds. This permits a small amount of jitter and delayed delivery due to buffering effects. Unverifiable beacons are dropped as CPL, which means that all Awareness Quality measurements in this chapter represent a combinatiorn of NPL and CPL. The sample rate for the collection of AQL measurements is fixed 
Table 13.1: Omission Schemes

\begin{tabular}{lcl}
\hline Name & Options & Abbreviaion \\
\hline Periodic Omission [17] & $\alpha=10$ & POoC-10 \\
Periodic Omission [17] & $\alpha=3$ [128] & POoC-3 \\
Neighbor-based [128] & - & NbCO \\
Congestion-based & Linear & CbCO-linear \\
Congestion-based & Quadratic & CbCO-quad \\
No omissions & - & NoOm
\end{tabular}

at 1 beacon interval, which is 100 milliseconds in our scenario. To test the efficiency of omission schemes under high loads, we scale the number of vehicles in the simulation scenario between 100 and 1500 vehicles on a $3 \mathrm{~km} \times 3 \mathrm{~km}$ mixed road network of the U.S. district of Suffolk county. We further specify two load scenarios:

- Low density: 300 vehicles, 33 vehicles $/ \mathrm{km}^{2}$

- High density: 1500 vehicles, 166 vehicles $/ \mathrm{km}^{2}$

A full simulation run executes 60 seconds of simulation time. During this time we do not simulate pseudonym changes. We expect the rate of uncoordinated pseudonym changes to be low enough to not be a relevant factor for the bandwidth optimization of beaconing services.

With the exception of Figure 13.8, which is a comparison of two very similar functions at a very large magnification level, the graphs in this chapter are generally shown without errors bars to enhance readability. The addition of small error bars to these graphs would not add to the understanding of the measured characteristics and would significantly impeded the legibility of the graphs.

\subsubsection{AQL Measurements}

We start by investigating awareness quality (AQL) measurement over multiple rings of safety areas. As described in Section 13.1, we specify ring shaped areas around vehicles. In our setting we follow [125] and define segments of $100 \mathrm{~m}$ width. The AQL measurement are calculated as the average over all vehicles in the scenario and over the whole 60 seconds of simulated time of the scenario. We collect measurements for $\mathrm{NbCO}$, two variants of $\mathrm{POoC}$, using a period of 3 and 10 for the omission scheme, and two variants of $\mathrm{CbCO}$, using a linear and a 
quadratic adaptation function. As a baseline we also measure the AQL for the NoOm scheme, which performs no omission.

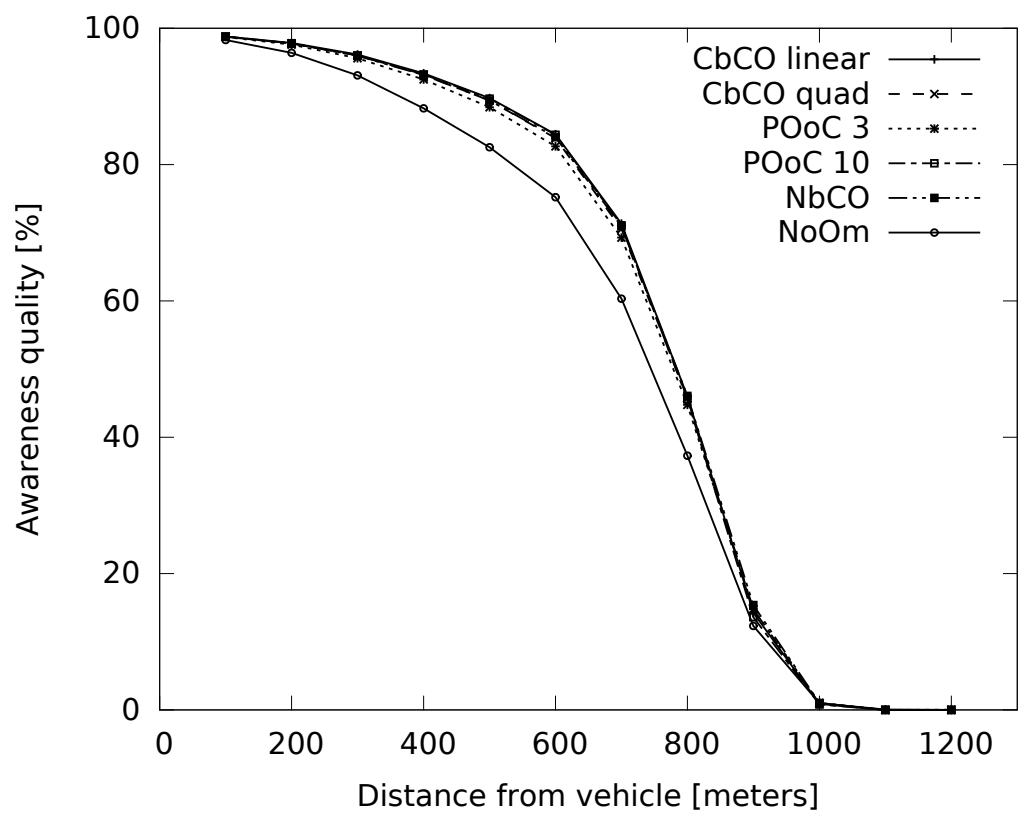

Figure 13.1: Average AQL in areas of $100 \mathrm{~m}$ width around vehicles in the low density scenario

In the low density scenario (Figure 13.1) the AQL starts out at almost $100 \%$ in the safety critical area up to $100 \mathrm{~m}$ around the vehicle. The AQL then gradually decreases with the distance of the rings from the vehicle until signal propagation attributes cause severe drops in the rings between $600 \mathrm{~m}$ and $900 \mathrm{~m}$ distance. AQL finally converges to $0 \%$ around the $1000 \mathrm{~m}$ ring. The baseline NoOm scheme stands out in this scenario as the only scheme with a reduced AQL compared to all omission schemes. This illustrates that even in this scenario with low density of vehicles we do see the negative effect of increased packet collisions due to consistently larger beacons. For the others schemes, this graph confirms what was already observed in the earlier analytical model in Chapter 11 and the simulation study based on packet loss in Chapter 12: Without congestion in the communication channel, any of the omission schemes performs equally well.

The same scenario in a high density configuration (Figure 13.2) shows this effect more visibly. The increase of packet loss decreases the AQL very quickly. None of the schemes manages to achieve an overall AQL above $70 \%$ in the safety relevant ring up to a distance of $300 \mathrm{~m}$. At the same time it is clearly visible that the various omission schemes show different scaling behavior in such a scenario. The $\mathrm{NbCO}$ and 


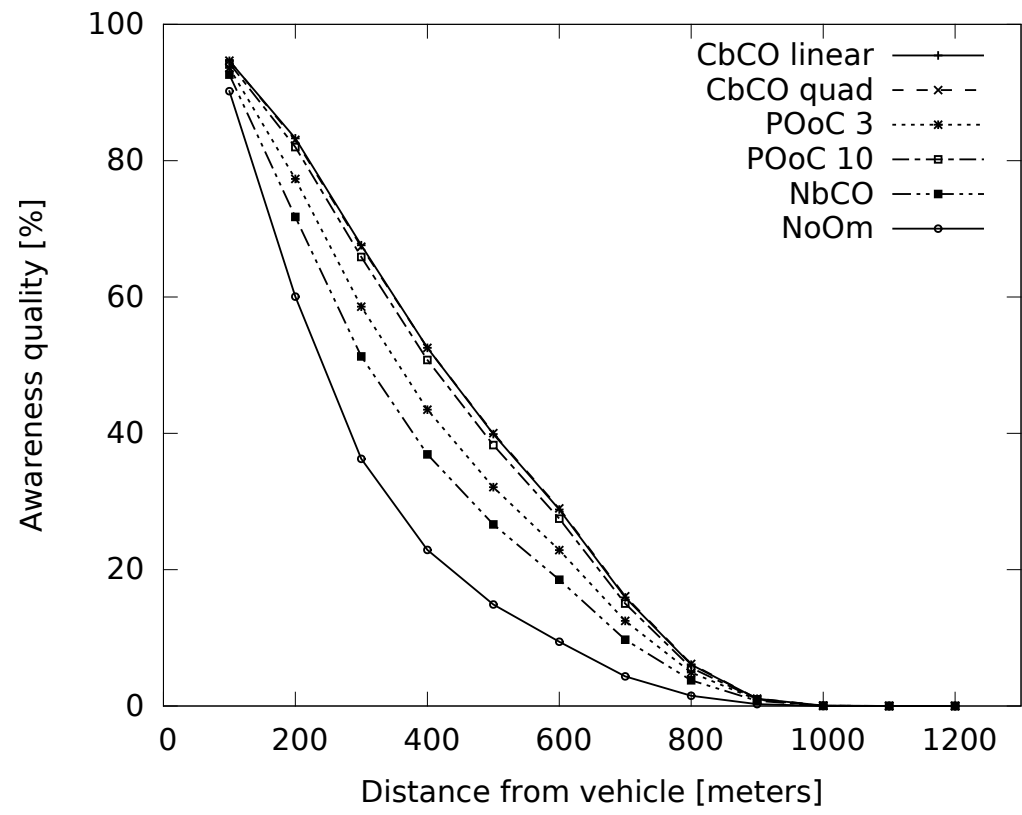

Figure 13.2: Average AQL in areas of $100 \mathrm{~m}$ width around vehicles in the high density scenario

$\mathrm{POoC}_{3}$ schemes in particular show worse performance than all other tested omission schemes. Only the very aggressive POoCio omission scheme shows performance that is similar to two $\mathrm{CbCO}$ schemes, which perform almost identically.

Overall, these measurements match expected results from previous investigations of AQL [126] and certificate omission schemes [105]. To investigate the scalability problem in further detail we simulate the AQL in function of the number of vehicles in the scenario. Figure 13.3 shows the corresponding graphs for all schemes in the safety critical ring of $0 \mathrm{~m}$ to $100 \mathrm{~m}$ around vehicles. The AQL measurements show very robust performance for all schemes in this area, which confirms the absence of regressions in all these schemes with respect to proper operation in this most critical area.

In Figure 13.4 the same scenario for the ring from $200 \mathrm{~m}$ to $300 \mathrm{~m}$ shows more diversified results. The region up to a distance of $300 \mathrm{~m}$ around a vehicle is not considered to be safety critical but still safety relevant [125]. Like in Figure 13.2, we see decreasing performance for the $\mathrm{POoC}_{3}, \mathrm{NbCO}$ and $\mathrm{NoOm}$ schemes under higher load in Figure 13.4. And again, the $\mathrm{CbCO}$ schemes show the best performance, with $\mathrm{POoC} 10$ as the only close contenter, which is what the $\mathrm{CbCO}$ schemes converge to under the highest levels of congestion. 


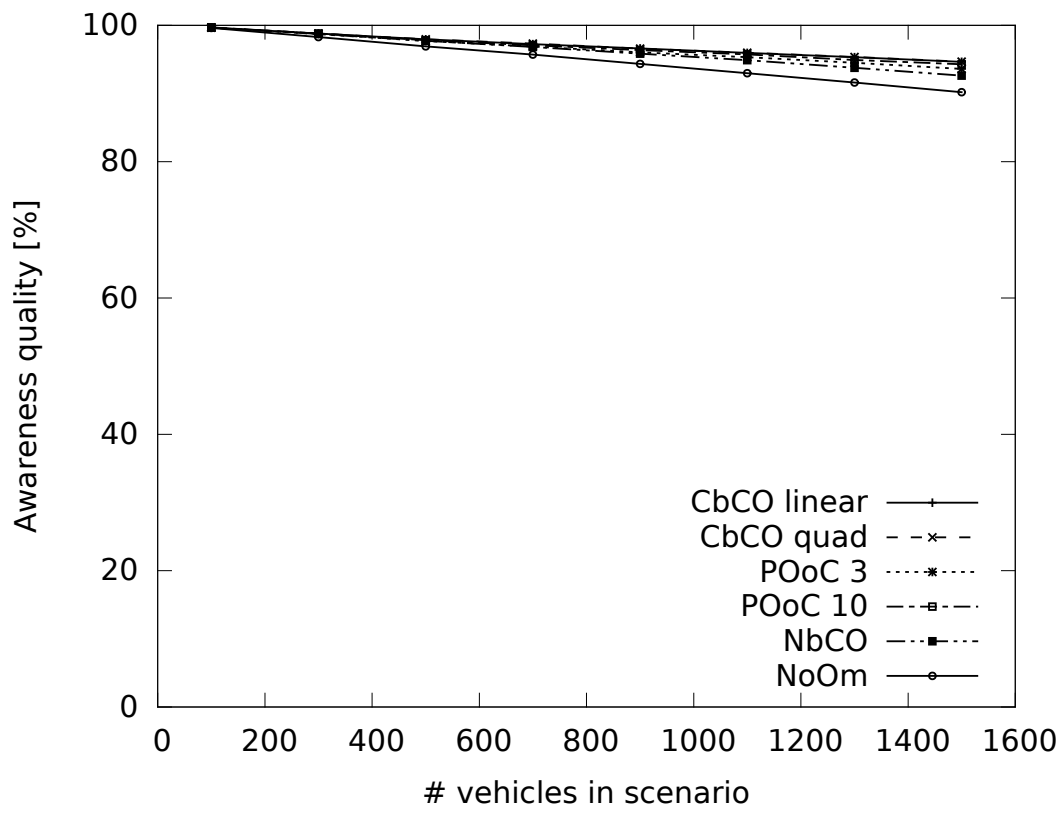

Figure 13.3: Average AQL for a safety area of $0 \mathrm{~m}$ to $100 \mathrm{~m}$ around vehicles under varying numbers of vehicles

\subsubsection{Time Series of AQL Measurements}

The results presented so far in Figures 13.1 - 13.4 illustrate a scalability problem of not using a certificate omission and of degraded performance of some certificate omission schemes in scenarios with high densities of vehicles. But based on these measurements it is hard to identify hidden patterns or different behavior in edge cases of the investigated schemes. In the preceding Figures we only see highly averaged AQL measurements, which got derived from an aggregation of samples collected over all vehicles in the scenario and over the whole 60 seconds of simulated time of the scenario. Previous research around certificate omission based on packet loss statistics faced similar problems. Even if the window of time that is used for the generation of measurements is small, the fundamental problem remains that we work with aggregated data.

The use of AQL as a metric enables us to avoid aggregation of measurements over time. Based on the sample rate of the AQL measurements it is possible to present AQL values as a time series of measurements. This is possible because AQL can easily report exact awareness quality values at any given point in time. Replicating this with packet delivery statistics would require rebuilding essentialy the 


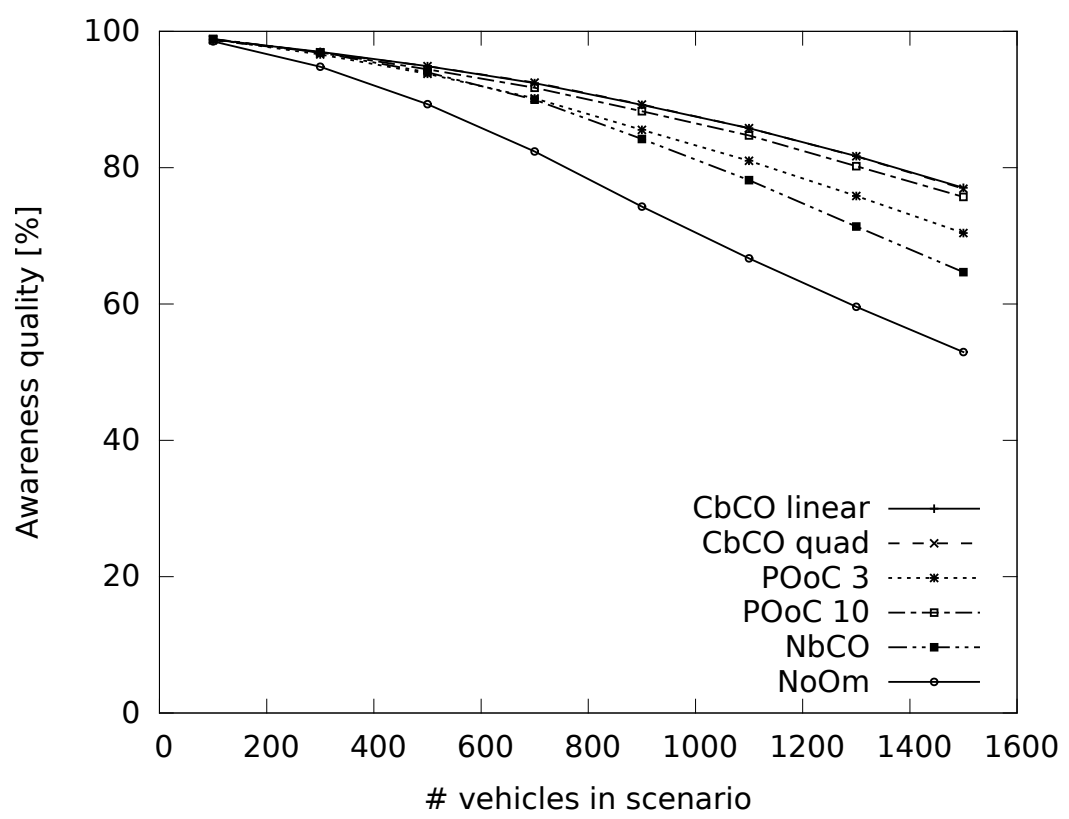

Figure 13.4: Average AQL for a safety area of $0 \mathrm{~m}$ to $300 \mathrm{~m}$ around vehicles under varying numbers of vehicles

entire awareness quality metric through tracking sender and receiver statistics over an interval interval of simulation time.

Figure 13.5 shows time series of AQL measurements, which are computed as an average over all vehicles in the scenario. Since no averaging takes place in the time domain we can see meaningful results at any given time in the simulation, even at very early stages. For better readability of the analysis we only show the initial 200 beacon periods of the simulation in Figure 13.5. A beacon period in our simulation scenario is specified as a fixed interval of $100 \mathrm{~ms}$ and 200 periods thus represent 20 seconds of simulated time. We choose the AQL sampling rate to match the beacon period. The scenario uses the high density configuration of 1500 vehicles and we use a ring of $300 \mathrm{~m}$ width from $0 \mathrm{~m}$ to $300 \mathrm{~m}$ distance around the vehicle in order to cover the entire security relevant area around the vehicle.

The later stages of the simulation, at the right side of the graph, converge to the globally expected behavior, which was already described earlier in this chapter and Chapter 11 and 12. But the early phase of the simulation reveals new details about the microscopic behavior of the omission schemes in the most challenging edge case for certificate omission: The sudden visibility of many unknown vehicles. This can occur for example when encountering the end of a traffic jam after a small hill. Such scenarios should be rare in practice, but the performance of 


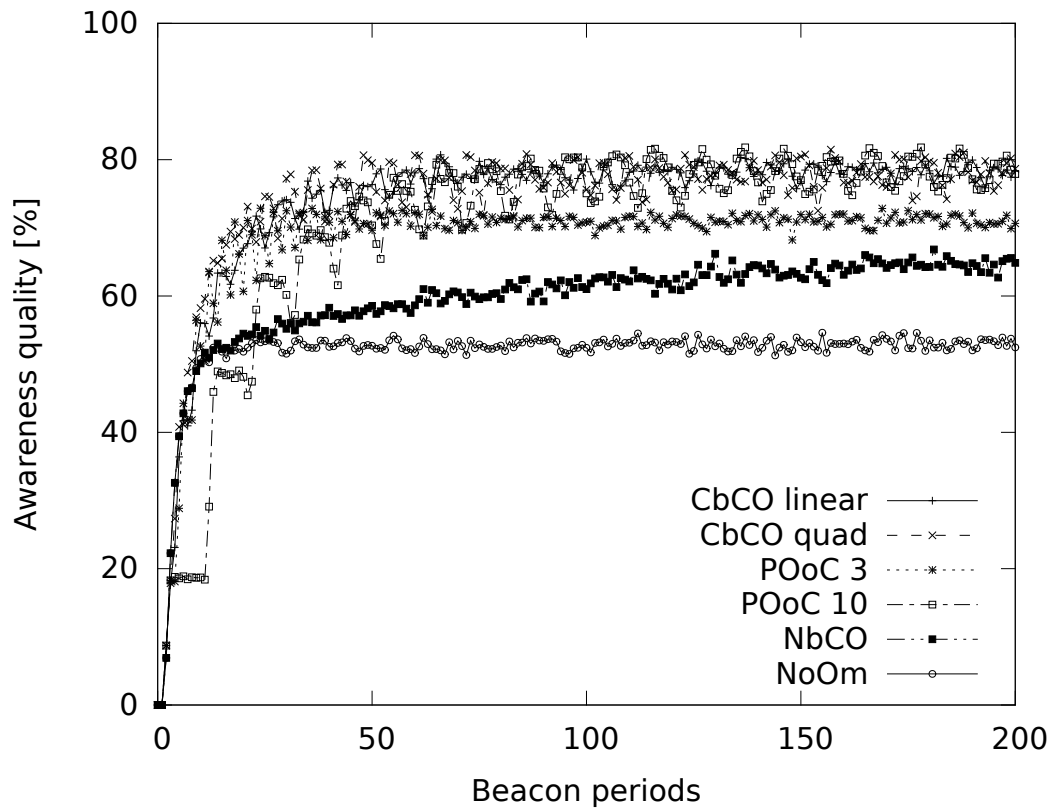

Figure 13.5: AQL measurement during the first 200 beacon periods of a high load simulation at a sampling rate of 1 per beacon cycle

certificate omission should be robust even in such worst-case scenarios and can in fact be critical in the avoidance of vehicle collisions.

The baseline performance is again represented by the NoOm scheme. We reiterate again that not using any omission at all is clearly detrimental to the overall performance of a secure beaconing service under high load. We notice that the $\mathrm{NbCO}$ scheme initially performs almost identical to the NoOm scheme. This suggests that it does indeed operate almost identically as the NoOm scheme. This behavior is explicable through the high amount of unknown neighbors in the early stages of the simulation. The existence of unknown neighbors in $\mathrm{NbCO}$ block omissions of certificates, which increases the load on the channel and the number of packet collisions. The $\mathrm{NbCO}$ scheme fails to reduce the load on the channel at a time when it would be most important to back off. Consequently it takes a comparatively long time for $\mathrm{NbCO}$ to escape from the default behavior of NoOm.

The $\mathrm{POoC}_{3}$ scheme, which we previously identified as the third scheme with significantly degraded scalability under high load, shows performance characteristics that are independent of the behavior of $\mathrm{NoOm}$ and $\mathrm{NbCO}$. The initial reaction time of $\mathrm{POoC}_{3}$ is competitive with other schemes, but $\mathrm{POoC}_{3}$ quickly stabilizes around an $\mathrm{AQL}$ level of approximately $70 \%$. This indicates that this fixed omission 


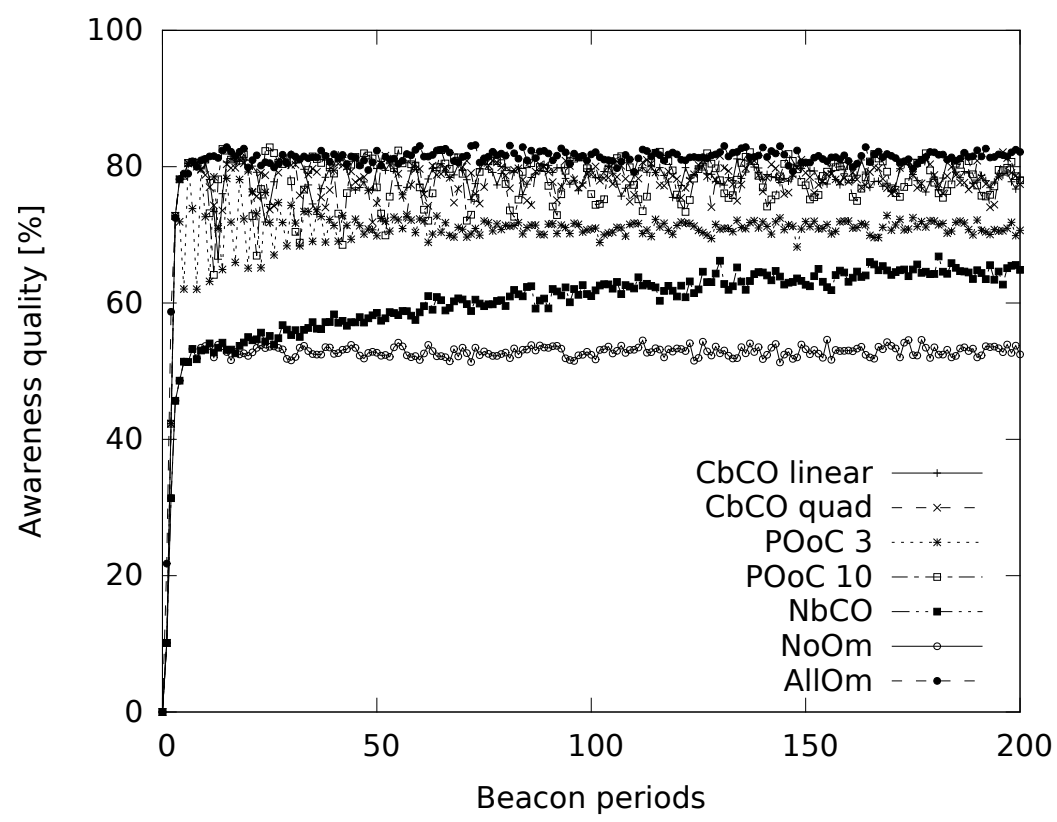

Figure 13.6: AQL measurement during the first 200 beacon periods of a high load simulation at a sampling rate of 1 per beacon cycle, not considering unverifiable packets as lost packets

works well during the initial pressure of exchanging many certificates, but during later stages it is obvious that this non-adaptive strategy leaves room for better scalability. A very notable difference can be seen between $\mathrm{POoC}_{3}$ and $\mathrm{POoC} 10$. The latter scheme shows very good overall scalability, matching the $\mathrm{CbCO}$ schemes. However, in the early stages of the simulation we can identify obvious problems in the reaction time of this scheme. The period of 9 omissions for every inclusion of a certificate is clearly visible in this figure. The AQL is clearly impacted by this long period of omissions. Every set of 10 beacon cycles represents a delay of one full second until CPL can be further reduced. Nevertheless, once the vehicles know the certificates of nearby vehicles, the scalability of POoCio is on par with the $\mathrm{CbCO}$ schemes, which use the available bandwidth optimally among the tested schemes.

To understand the impact of verifiable packets, in particular with respect to the POoC schemes, it is useful to look at the same results without discarding unverifiable packets. Figure 13.6 shows the resulting graph for such an analysis. In this figure we also show an omission scheme that omits all certificates, called AllOm. Under normal circumstances this would of course lead to an AQL of $\mathrm{o} \%$, because certificates 


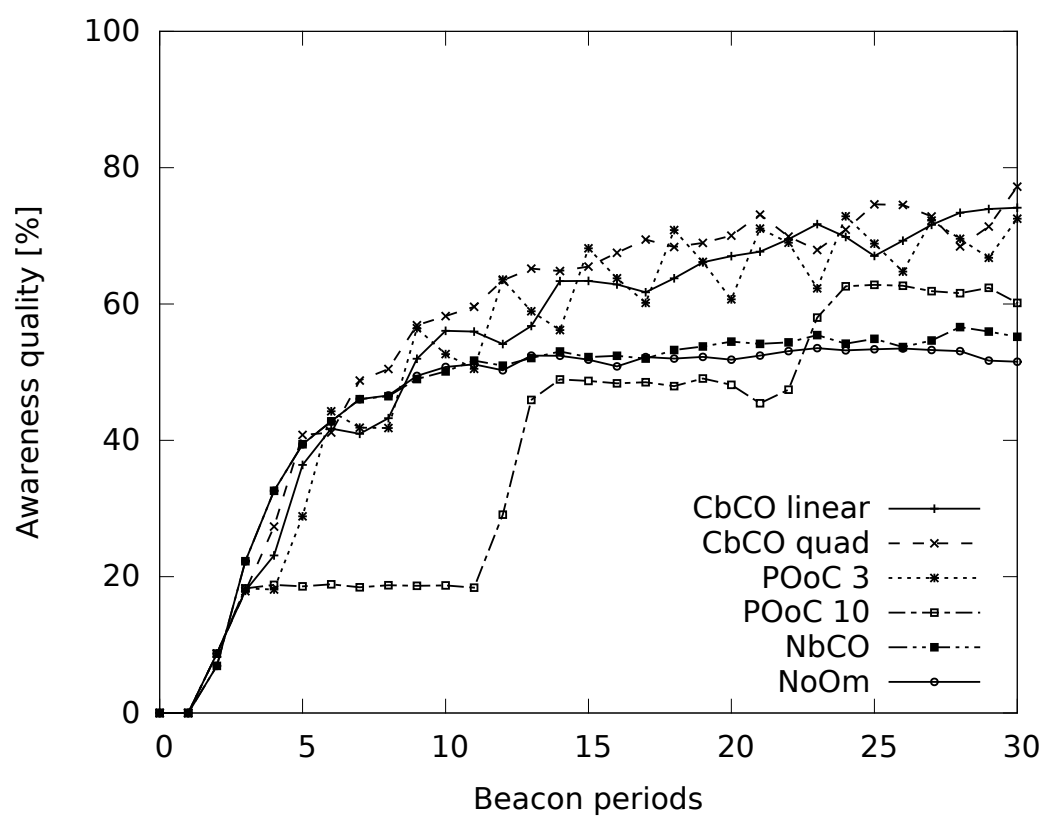

Figure 13.7: AQL measurement during the first 30 beacon periods of a high load simulation at a sampling rate of 1 per beacon cycle

never get exchanged between vehicles. But it is useful to see this scheme here as an upper bound.

We see NoOm and $\mathrm{NbCO}$ almost unaffected by cryptographic packet loss (CPL), which indicates that these schemes are dominated by regular network packet loss (NPL). In fact, we can see that the scalability attributes of all schemes eventually get dominated by NPL effects, as the AQL measurements converge to the same values as in Figure 13.5. But the reaction time at the beginning of the simulation shows critical differences. We see that the "stairs effect" of POoC1o as seen previously is eliminated if we do not consider CPL. Instead, we see almost optimal behavior approaching that of AllOm, with the exception of occasionally dropping down due to the inclusion of certificates in every 1oth beacon cycle. The $\mathrm{CbCO}$ schemes notably perform very similar to POoCio in this Figure, indicating that the pure NPL tradeoff is as effective as POoC10. This is clear, since the $\mathrm{CbCO}$ schemes are algorithmically limited to behavior like POoCio under high load [48]. Overall, we can attest that the $\mathrm{CbCO}$ omission schemes perform as well as the POoCio omission scheme, while avoiding the deficiency of risking avoidable packet loss during the first encounter of a new vehicle. All other omission schemes converge to generally weaker performance. 


\subsubsection{Optimal Certificate Omission Scheme}

The ability to zoom in on the early stages of the simulation and to see exact quality measurements at the selected sampling rate allowed us to derive a much better understanding of the behavior of the schemes. But so far we could not see useful information about the behavior of the two $\mathrm{CbCO}$ schemes, beyond the observation that the schemes perform very well. In Figure 13.7 we zoom in even further by reducing the observation window to the first 30 beacon periods, which is equivalent to 3 seconds of simulation time at our selected beaconing interval of $100 \mathrm{~ms}$. Again we notice the "stairs effect" of POoCio and the very similar behavior of the $\mathrm{NoOm}$ and $\mathrm{NbCO}$ schemes. Interestingly the $\mathrm{POoC}_{3}$ scheme performs very well at this early stage of the scenario, indicating that it strikes a good balance between reducing load on the communication channel and disseminating certificates to reduce CPL. We can also clearly see the oscillation of $\mathrm{POoC}_{3}$ on a period of 3 .

The two $\mathrm{CbCO}$ schemes seem to exhibit a similar oscillation pattern as $\mathrm{POoC}_{3}$ at this stage of the simulation. The $\mathrm{CbCO}$ schemes do not use a fixed omission though. The observed behavior can be explained by the fact that the $\mathrm{CbCO}$ schemes are adaptive POoC schemes. The schemes start out with empty neighbor tables, indicating that each vehicle is free to include certificates in beacons, since the channel is assumed to be free. With the exception of POoCio all schemes perform similarly up to beacon period 6. Ignoring $\mathrm{POoC} 10$, the performance is tightly bounded by the $\mathrm{NoOm}$ and $\mathrm{POoC}_{3}$ schemes, indicating that we see very few omissions at this point. After the 6 beacon period mark we see a split into two groups. While $\mathrm{NoOm}$ and $\mathrm{NbCO}$ remain stagnant, we see the $\mathrm{CbCO}$ schemes perform similarly well as the fixed $\mathrm{POoC}_{3}$ scheme, indicating that these schemes continue to act similarly. The explanation for this can be found in the neighbor tables that slowly build up in the vehicles and gradually adjust the omission period to higher levels. Neighboring vehicles that send unverifiable beacons are not added to the neighbor table, leading the $\mathrm{CbCO}$ schemes to keep the omission rate at a low value. With this behavior the $\mathrm{CbCO}$ schemes apparently strike the best balance between NPL and CPL. Starting out with no omission and gradually increasing the omission rate only if two conditions are met:

- There are many neighbor vehicles around the vehicle, implying that the communication channel is congested,

- The neighbors send verifiable beacons, implying that the vehicles know each other and certificate omission will not have a negative effect.

A remaining uncertainty is the competitive behavior of the two $\mathrm{CbCO}$ scheme amongst each other. To find an answer to this question 


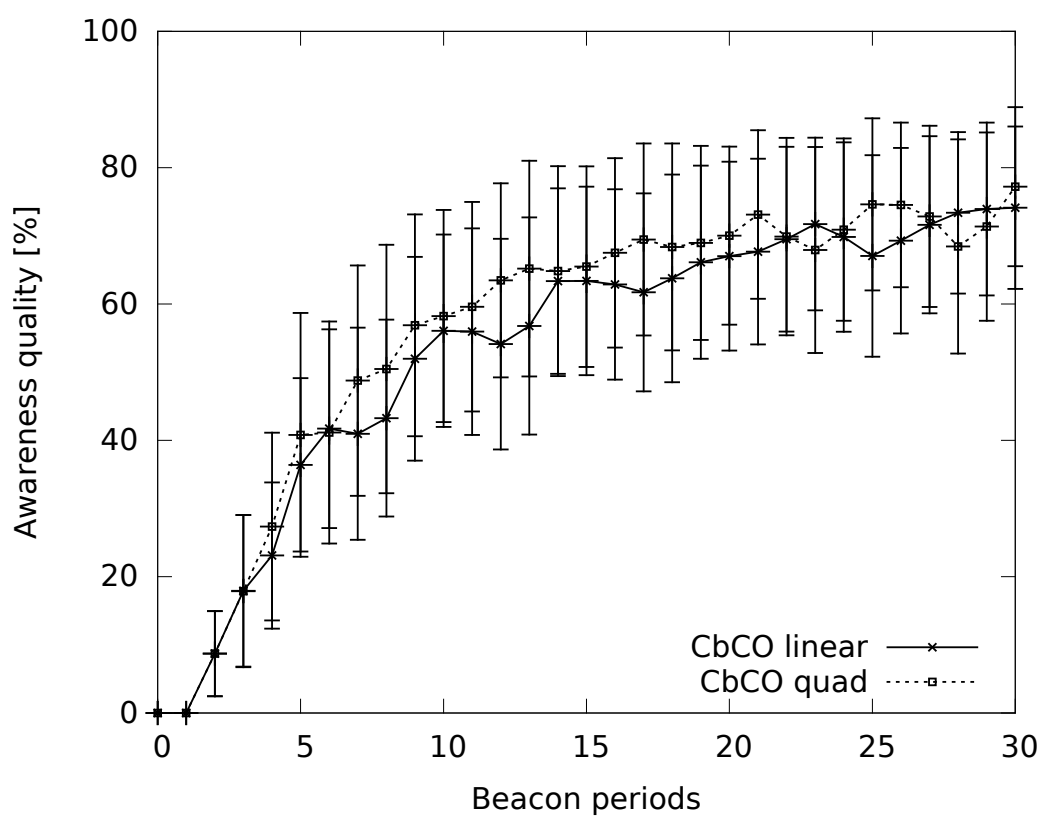

Figure 13.8: Comparative AQL measurement of $\mathrm{CbCO}$ linear and $\mathrm{CbCO}$ quad during the first 30 beacon periods of a high load simulation at a sampling rate of 1 per beacon cycle

we isolate the two graphs for linear $\mathrm{CbCO}$ and quadratic $\mathrm{CbCO}$ in Figure 13.8. We know that these two schemes converge to very similar scalability properties overall. In terms of reaction time we can however identify small differences. The use of a more aggressive quadratic adaptation function leads to better reaction times in a situation with many new neighbors appearing around a vehicle. In this Figure we also show error bars for the AQL measurement to illustrate the spread of AQL over the vehicles in the scenario. Since our simulation was configured with a beacon lifetime of less than two beacon periods we see every single lost beacon as a degradation of the AQL. The high standard deviation of up to $20 \%$ is the result.

Finally, with the availability of AQL as a fine-grained and exact way to investigate edge-cases of certificate omission, we show another edge case that indicates $\mathrm{CbCO}$ quad to be superior to $\mathrm{CbCo}$ linear. In Figure 13.9 we show the first 30 beacon periods of a scenario with a low density of cars. It is expected that all schemes perform very well in this scenario, with Figure 13.3 suggesting that we should see AQL values around $98 \%$. But we discovered that some schemes struggle in the first couple of beacon periods, as seen in Figures 13.6 and 13.9. The $\mathrm{NoOm}$ and $\mathrm{NbCO}$ perform best under these circumstances, which are purely defined by $\mathrm{CPL}$, followed by $\mathrm{CbCO}$ quad, $\mathrm{POoC}_{3}, \mathrm{CbCO}$ linear 


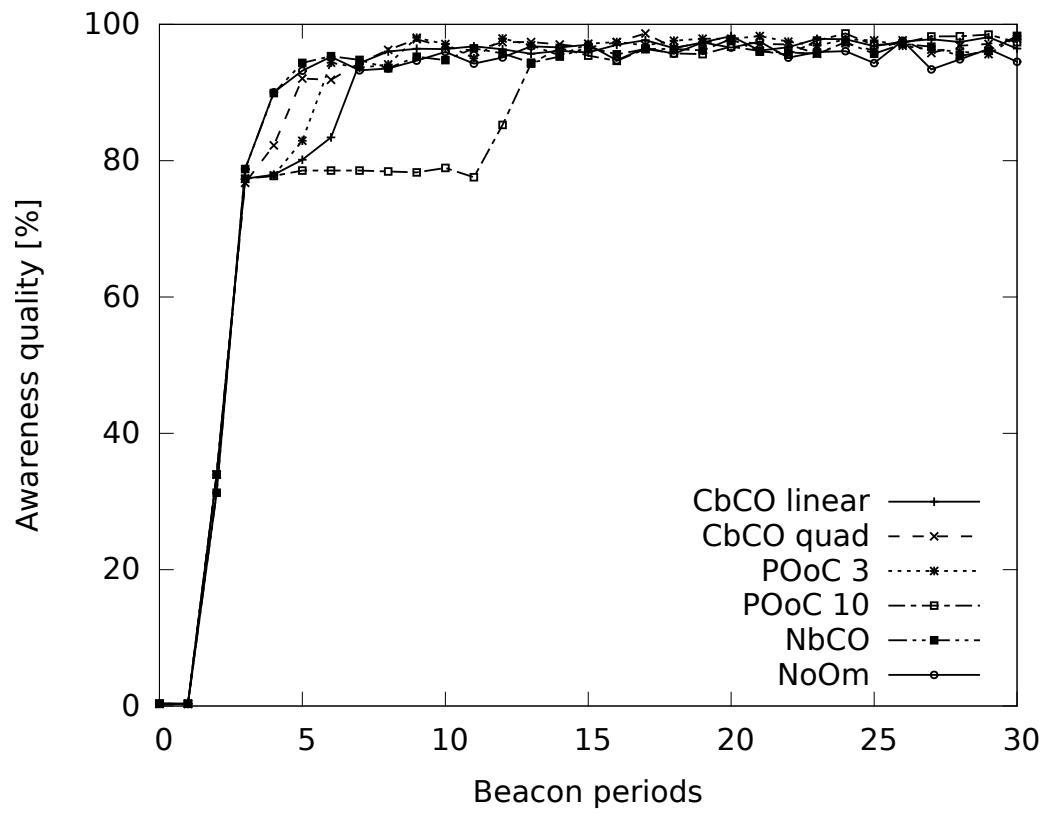

Figure 13.9: AQL measurement during the first 30 beacon periods of a low load simulation at a sampling rate of 1 per beacon cycle

and trailed by POoC10. The POoC1o scheme clearly exhibits a "stairs effect" with CPL delays of multiples of 10 beacons or 1 second. This deficiency of POoC10 only occurs during a short first encounter period, but it occurs even under low load and is avoidable: The $\mathrm{CbCO}$ schemes adapt their omission period to show essentially the same or slightly better scalability properties as POoCio under load, while effectively avoiding the risk of encountering this CPL deficiency. Among the $\mathrm{CbCO}$ schemes, the configuration with a quadratic adaptation function clearly beats the performance of $\mathrm{CbCO}$ linear in this edge case.

We conclude that, thanks to AQL measurements we could identify quadratic $\mathrm{CbCO}$ as the preferred choice in high load scenarios and as a near optimal choice for low load scenarios. Our results are summarized in Table 13.2. 
Table 13.2: Performance of Omission Schemes

\begin{tabular}{lcl}
\hline Name & Reactivity & Scalability \\
\hline No omissions (NoOm) & ++ & -- \\
Neighbor-based (NbCO) & ++ & - \\
Periodic Omission (POoC-3) & + & - \\
Periodic Omission (POoC-10) & -- & ++ \\
Congestion-based (CbCO-linear) & + & ++ \\
Congestion-based (CbCO-quad) & ++ & ++
\end{tabular}




\section{4}

CERTIFICATE PRE-DISTRIBUTION

The fundamental trade-off of bandwidth saving certificate omission schemes, which we investigated in the preceding chapters, is the introduction of cryptographic packet loss (CPL) in the form of unverifiable packets. Omission schemes need to balance the intended decrease of network packet loss (NPL) as a result of fewer collisions in the communication channel against the unintended introduction of CPL. Waiting periods for exchanges of certificates after the first encounter of a vehicle in communication range need to be minimized to reduce CPL.

In this chapter propose to combine omission schemes with predistribution, a technique to reduce CPL while maintaining the benefits of omission schemes. Pre-distribution anticipates the need for certificates and disseminates them proactively. Needs for certificates arise through the arrival of new vehicles in a geographic region, or through a switch of cryptographic identities with the intention of breaking long term linkability of vehicle movements over extended periods of time. We consider two utility classes of pre-distribution techniques, geographic and temporal pre-distribution, as these are the dimensions that define the reasons for pre-distribution to be effective. Pre-distribution of certificates, to the best of our knowledge, was not proposed as a technique for certificate management in Vehicular Ad hoc Networks (VANET).

\subsection{AN INITIAL ASSESSMENT}

For an evaluation of the concept of pre-distribution we perform another simulation study. The basic parameters of the simulation correspond to the common settings that were introduced in Chapter 11. We deviate from the usual Suffolk county scenario, which served as the reference scenario in most certificate omission studies since [128], and instead use a synthetic scenario to more precisely investigate the effects of our two types of pre-distribution patterns. In order to trigger certificate omission with the context adaptive $\mathrm{CbCO}$ scheme, we need clusters of vehicles with to create actual congestion. To achieve this effect we define two clusters of vehicles at opposing ends of a $2.5 \mathrm{~km}$ by 
$1 \mathrm{~km}$ map. A total of 300 vehicles are initially placed on the map that features a simple layout of two main roads that meet at a central 4way intersection. The two clusters of vehicles move towards the center and the vehicles depart the simulation in all four possible directions after crossing the intersection. The simulation results are based on the average of 5 runs that simulate 150 seconds of simulation time. The mobility patterns were generated with the VanetMobiSim [59] tool.

The pre-distribtion technique can be implemented in several variations, which can be categorized into two main degrees of freedom. The first category concerns the question when and how to pre-distribute. The second category concerns the question what to pre-distribute. We first explain our reasoning for when and how to pre-distribute certificates.

In this initial assessment of pre-distribution we limit ourselves to direct 1-hop distribution of certificates and make the pre-distribution decisions exclusively within the sending vehicle. This keeps uncertainty and thus the potential for suboptimal pre-distribution decisions low. It is possible to imagine that pre-distribution can be requested by third party vehicles. An example could be a vehicle that travels toward a waypoint and sends a message over a multi-hop routing services towards this waypoint, which triggers pre-distribution of certificates that might be relevant along this path. Such a system requires multi-hop routing infrastructure, which should ideally support geographical routing towards a target area, which is an assumption that is unlikely to be applicable at least for the first generation of vehicular communication deployments. Alternatively a centralized routing and distribution service, for example via the cell phone network, could be used. However, having such an infrastructure in place and available at any time would conceivably make $\mathrm{V}_{2} \mathrm{~V}$ pre-distribution redundant, since this infrastructure could itself be used as another communication channel with independent congestion patterns. While we not that such infrastructure would be greatly beneficial, we do not consider such solutions in this assessment. As we limit our investigation purely to $\mathrm{V} 2 \mathrm{~V}$ communication, a main limitation is be the messaging overhead of pre-distribution requests. Pre-distribution is a counter-measure against cryptographic packet loss, which is introduced is an undesirable sideeffect of certificate omission. The reason to perform certificate omission is to save bandwidth in a potentially congested communication channel. If forwarding of certificate pre-distribution request was allowed, it would create additional non-critical messages in a communication channel with limited bandwidth, which should be reserved for critical messages. By definition, the vehicle that requests pre-distribution of certificates does not know the vehicles in the targeted area, which implies that there could be congestion and the request message would be detrimental for overall packet delivery success. A quality of service 


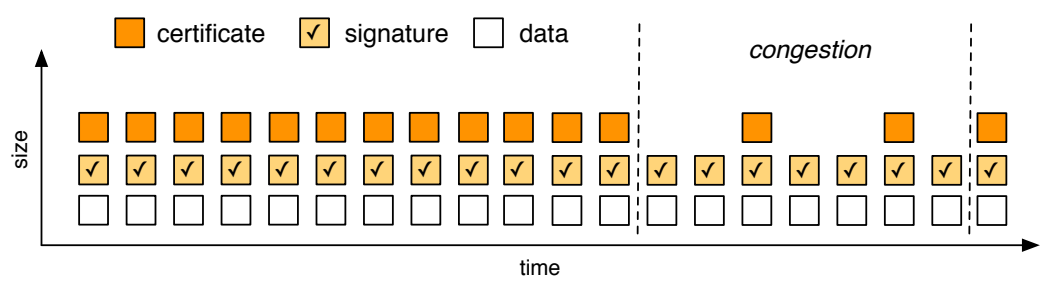

Figure 14.1: Congestion-based Certificate Omission reduces inclusion of certificates when congestion in the communication channel increases

system with differently prioritized queues could help to alleviate the negative effects of unnecessary low priority messages by dropping noncritical messages during times of congestion. However, this only work on the sender side. On the receiver side in a congested area we could still potentially experience a degradation of service quality. Finally, there is also the potential to use certificate pre-distribution requests as an traffic augmentation option in distributed denial of service attacks. These concerns lead us to only consider 1-hop distribution and to make the pre-distribution decisions only locally.

After establishing that our pre-distribution assessment only considers local proactive decision making and no multi-hop reactive predistribution, we still identify another option to vary the question of when to pre-distribute certificates: As explained above, the benefit of pre-distribution lies in he elimination of cryptographic packet loss, which can be caused as a side-effect of certificate omission. We can assume a certificate omission strategy to be active in the scenarios that will be applicable to certificate pre-distribution. This presents three options for the inclusion of pre-distribution certificates. Either as a concatenation with a regularly included certificate, as a replacement of a regularly included certificate, or in the gaps that are created by omission of certificates.

In the Congestion-based Certificate Omission ( $\mathrm{CbCO}$ ) scheme, which we identified as the most effective scheme in previous chapters, we see omission only during times of congestion in the communication channel. This is illustrated in Figure 14.1. Using these gaps for predistribution implies that this happens during periods of high congestion, which would completely negate the purpose introducing certificate omission at all. This leaves us with concatenation and replacement of certificates as viable strategies. We select concatenation for our investigation, which is intuitively the safer option. Assuming $\mathrm{CbCO}$ as an underlying certificate omission scheme, it is relatively safe to assume that certificates are only attached when congestion levels are low. This implies that it is also relatively safe to have slightly larger payload lengths for concatenated certificates. If on the other hand a certificate 


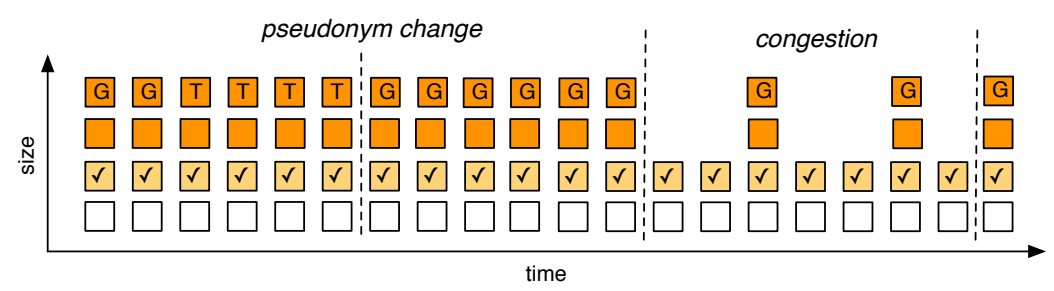

Figure 14.2: Geographic (G) and Temporal (T) certificate predistribution through certificate concatenation on top of Congestion-based Certificate Omission

gets sent during congestion, this implies that this is absolutely necessary to uphold minimum inclusion limits. In this case it would not be permissible to replace the included certificate with a pre-distribution certificate. For these reasons we prefer the strategy of concatenation over replacement, as shown in Figure 14.2.

The overall length of such messages corresponds to the composition of position beacons that was described in Chapter 12, with the certificate component doubled. This leads to message lengths of 115 bytes for messages without any certificates and 395 bytes for messages with two certificates attached.

Next we discuss the question what to pre-distribute, which leads us to the aforementioned utility classes of geographic and temporal pre-distribution. We recall that we limit this assessment to proactive single-hop dissemination of certificates. This greatly reduced the set of certificates that might useful to immediate neighbors, since the difference in awareness of vehicles between a sender vehicles and potential receiver vehicles will be relatively small. A vehicle should select a certificate for pre-distribution which will be useful for the maximum number of neighbors. This would imply prioritizing a certificate of a neighbor that just entered communication range or selecting a certificate of a neighbor that is at the edge of communication range if another vehicle is at a diametrically opposing edge. Ideally a vehicle could take the travel trajectory into consideration. The certificate of a vehicle that is traveling in the opposite direction as a vehicle is unlikely to be useful to other vehicles that travel in the same lane. However, it might be especially useful for pre-distribution to vehicles on the lane of the vehicles traveling in this opposing direction. These are exactly the benefits of geographical pre-distribution.

The selection of candidate certificates for pre-distribution is an open field for investigation of useful strategies, but these strategies are very likely to require application level interpretation of vehicle movement. For the purposes of our initial assessment we limit our investigation to a random selection of a current neighboring vehicle. Current in this context is defined by the beacon lifetime period of two beaconing peri- 
ods, or 200 milliseconds. The investigation of more enhanced selection strategies is left as future work.

The other utility class of pre-distribution of certificate concerns temporal pre-distribution effects. These effects reduce cryptographic packet loss through the pre-distribution of certificates that will be useful to neighbors not due to the mobility of nodes, but due to changes of certificate material. This is expected to occur mainly due to the change of pseudonymous identities with the purposed of protecting privacy by breaking long-term and mid-term linkability of movement patterns to specific vehicles. Such pseudonym changes might occur in synchronization with large numbers of neighbors in order to maximize uncertainty for an attacker. Further discussions of pseudonym change strategies can be found in [108].

The selection of certificates with utility for temporal pre-distribution is straightforward. During a period before a pseudonym change a vehicle can start to pre-distribute it's future certificate material. This period should not be too long from the switch to a new pseudonym in order to reach only the relevant neighboring vehicles. We selected a period of 20 beacon cycles, which is equivalent to 2 seconds at $10 \mathrm{~Hz}$ beacon frequency, as our time window for temporal pre-distribution. Figure 14.2 shows an illustration of our pre-distribution technique, featuring pre-distribution certificates, which are sent for geographic effects, and pre-distribution certificates, which are sent for temporal effects ahead of a pseudonym change.

The receivers of temporally pre-distributed certificates could use these to link an old and new pseudonym. An option to reduce this ability is to ensure that the distribution frequency of certificates that get pre-distributed for temporal gains is indistinguishable from that of certificates that are pre-distributed for geographical benefits. However, in general it is an intended goal of broadcasting position beacons, that short-term linkability should be seamless for reasons of guaranteeing safety goals. Therefore, vehicles in the immediate neighborhood, and only those, should be able to track vehicles across pseudonym changes. We point out again, that [108] provides an extensive discussion of pseudonym strategies.

For our purposes we go one step further with this assumption and consider a coordinated pseudonym change of a large number of vehicles. This presumes that vehicles exchange and synchronize their intention to change their pseudonyms with the aim of maximizing uncertainty for outside observers. For simplicity we do however skip a discussion or implementation of synchronization protocols. Instead, we schedule a synchronized pseudonym change between the 6oth and $65^{\text {th }}$ beacon cycle. This represents a 6 second offset into the simulation time, which is enough enough warm up time for the service quality metrics to settle. 


\begin{tabular}{|c|c|}
\hline Parameter & Value \\
\hline Certificate omission scheme & CbCO \\
CbCO configuration & Quadratic \\
Attachment strategy & Concatenation \\
Number of pre-distribution certificates & 1 \\
Pre-distribution distance & Single hop \\
Temporal period & 2o beacon cycles \\
Geographic selection & Randomized \\
\hline
\end{tabular}

Table 14.1: Pre-distribution settings

As our main metric we again chose abstract application service quality a measured by Awareness Quality, which was previously introduced in Chapter 13. For the calculation of Awareness Quality we consider a single relevant area covering the entire communication range around each vehicle. The sample rate of the Awareness Quality recordings is fixed a 100 milliseconds, which translates to 1 beacon cycle at our selected beaconing frequency of $10 \mathrm{~Hz}$. The overall set of our selected pre-distribution settings is summarized in Table 14.1.

In Figure 14.3 we show the averaged Awareness Quality ratings for all vehicles over the course of the simulation. A pre-distribution strategy that exclusively uses geographic pre-distribution combined with a $\mathrm{CbCO}$ certificate omission strategy is shown in comparison to a regular $\mathrm{CbCO}$ certificate omission strategy without pre-distribution. We see an initial ramp up at the beginning of the simulation, which is a period of high cryptographic packet loss, as no vehicles know any other vehicle. This warm up phase shows is very sensitive to packet loss, as any lost packet during this phase leads not only to one missed position beacon but potentially to several missed beacons until a position beacon with a certificate is received. It is highly insightful to study the positive and negative effects of certificate omission in such a phase, as the effects of good or bad decisions are potentially highly visible during this period. We refer to Chapter 13 for deeper analyses and interpretations of the effects of certificate omission.

We note that pre-distribution for purely geographical benefits performs slightly worse during the warm-up phase of the simulation than not performing any pre-distribution. The slower uptake of Awareness Quality can be explained through the significantly larger payload length of the broadcasted message, which lead to a higher likelihood of network packet loss due to congestion or collisions. A peculiarity of the warm-up phase is the fact that all vehicles start with an initial attachment of one certificate, which amplifies the negative effects of 


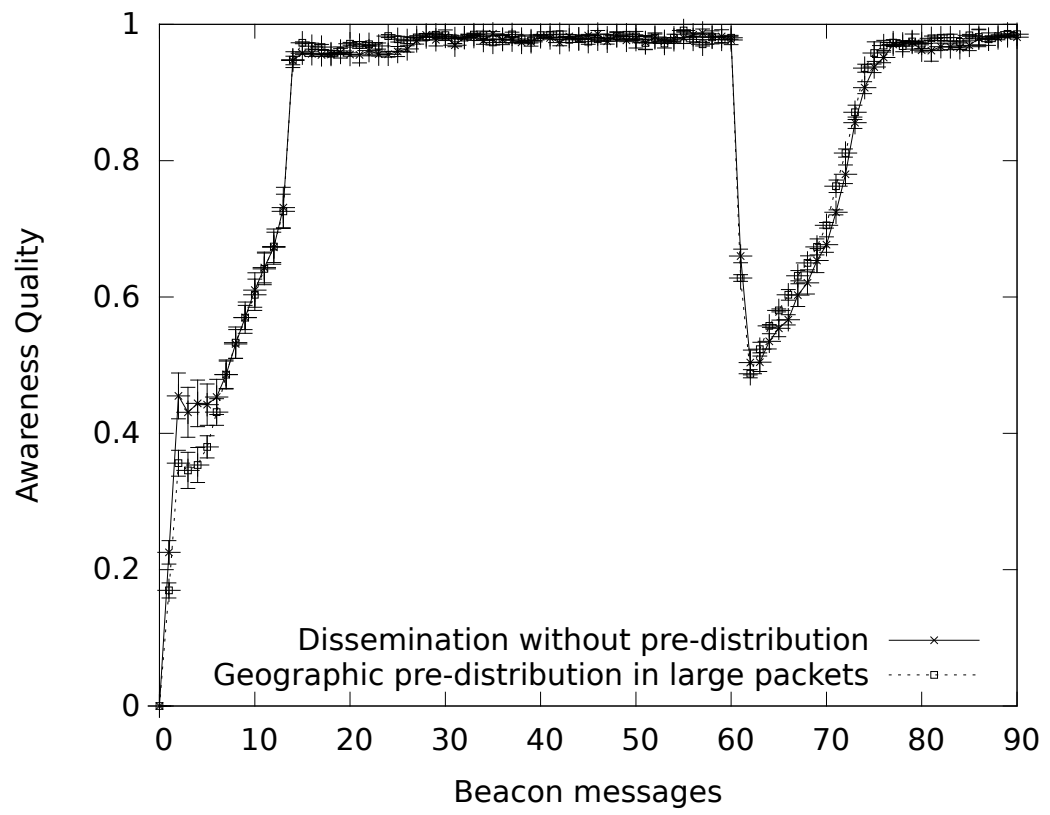

Figure 14.3: Awareness quality without and with geographic predistribution

any chance of increased network packet loss. Additionally, the vehicles need to build up a neighbor tables to collect knowledge of the congestion level in the environment, which means that vehicles initially underestimate the congestion in the communication channel, which, in the case of $\mathrm{CbCO}$ leads to fewer omissions than would be expected with better knowledge of the true congestion levels.

However, these peculiarities cannot alter the conclusion that no positive effects of purely geographic pre-distribution countered the negative effects of enlarged payload lengths. As the Awareness Quality ratings quickly converge to a stable level around $95 \%$ we point out that the scenario of two clusters of vehicles meeting at a central intersection quickly leads to a situation where all vehicles are in communication range of each other. In this situation geographic pre-distribution cannot deliver benefits.

We derive from Figure 14.3 that benefits from purely geographical pre-distribution over single-hop do not justify the larger payload lengths. We do however see that pre-distribution also does not cause a measurable difference in Awareness Quality during the regular uneventful phases of our simulation scenario. During these stages the extended payload length apparently do not cause degradations of service quality. Additionally, geographic pre-distribution seems to have a small positive effect during the recovery process from the coordi- 
nated pseudonym change at beacon interval 6o. Both effects might be attributable to the fact that the $\mathrm{CbCO}$ scheme at later stages of the simulation scenario is successful at reducing channel congestion, which eliminates the negative effect of extended payload lengths that us caused by the pre-distribution technique. This way it is possible for a small positive effect of geographic pre-distribution to be visible. However, the difference are too small and to short to allow a conclusive result.

Future work might investigate if more careful selection of predistribution certificates or multi-hop dissemination techniques in a larger scenario could create enough benefit to make geographic predistribution worthwhile. Another possibility could be a strategy to reduce the amount of pre-distribution in a way that not all position beacons with certificates include the double sized pre-distribution certificate. A differentiation would be necessary to detect situations where the successful distribution of the senders certificate is more important than pre-distribution. This would be the case in situations of overall congestion, while pre-distribution makes more sense when just a small number of vehicles newly arrives in a scenario and would benefit from pre-distribution.

In Figure 14.4 we see the second approach to certificate pre-distribution applied to the same scenario. In this case we only see pre-distribution of future certificate of the sender vehicle. And this only occurs during a short period before a pseudonym change. We see that there is effectively no difference in the Awareness Quality performance of $\mathrm{CbCO}$ combined with temporal pre-distribution compared to $\mathrm{CbCO}$ alone up to beacon period 60. This is clear until beacon period 40 , because in fact there is no difference between the two methods until that point. The fact that there is no discernible difference between the two method between beacon period 40 and 60 is remarkable, because this is the period when temporal pre-distribution starts to become active. The fact that we see no difference in Awareness Quality indicates that network packet loss is low enough to not cause the enlarged payload length due to the temporal pre-distribution to be a measurable problem.

At the time of the coordinated pseudonym change we see a drastic benefit of temporal pre-distribution. Due to the pre-distribution of the new pseudonymous certificate material the Awareness Quality only drop to $80 \%$ instead of $50 \%$, as it does without temporal predistribution. While this means that we still see a small decrease in Awareness Quality the decrease is far less severe. And Awareness Quality is back to the regular level of around 95\% after just 4 beacon cycles. It take between 15 and 25 beacons cycles for the Awareness Quality to return to these levels without temporal pre-distribution. At the beaconing frequency of $10 \mathrm{~Hz}$ these values represent 0,4 seconds compared to 1,5 to 2,5 seconds. This clearly indicates that temporal 


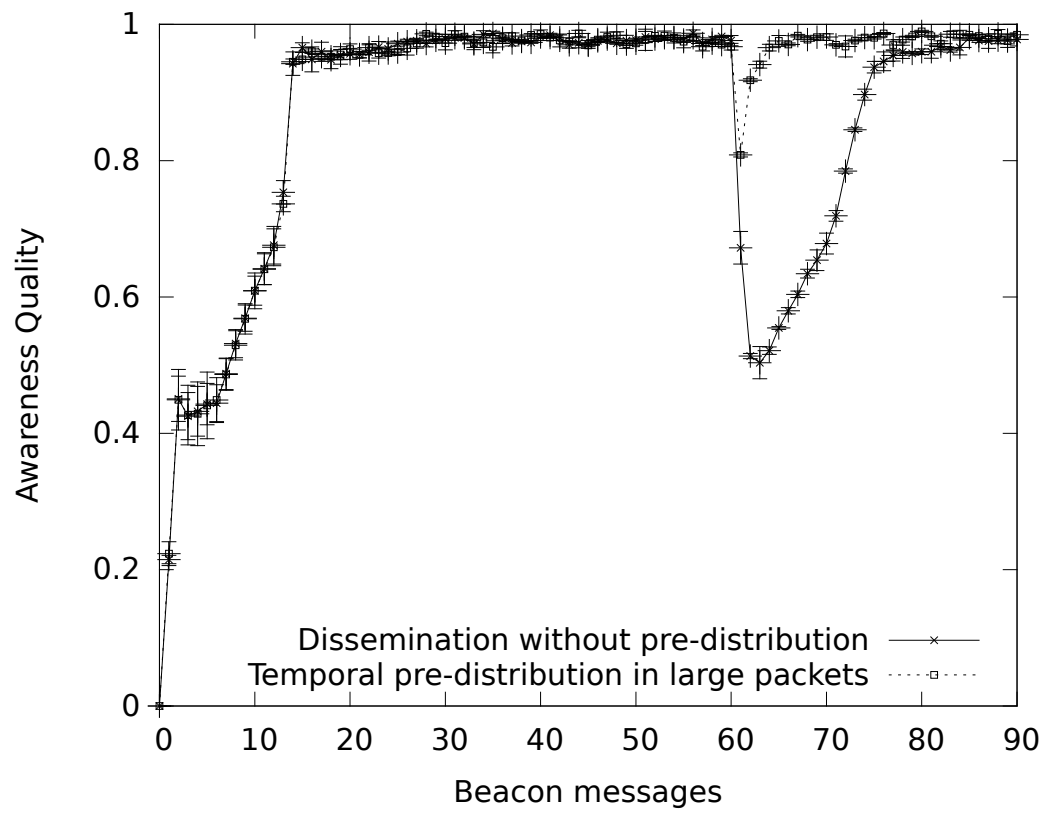

Figure 14.4: Awareness quality without and with temporal predistribution

pre-distribution could almost entirely eliminate the negative effects of changing a pseudonym on cooperative awareness.

Finally, in Figure 14.5 we show the combination of the geographical and temporal pre-distribution technique, which most closely corresponds to the illustration in Figure 14.2. The combination of the two utility classes is useful primarily under the assumption that both techniques might show benefits. Another motivation to combine the techniques is to increase uncertainty for an observer, who could ideally not differentiate between certificates that are pre-distributions for the goal of geographical benefits or as a pre-distribution of a future certificate with the aim of achieving temporal pre-distribution.

The mix of the two techniques shows a mix of the two previously observed effects. Until the coordinated pseudonym change we essentially see only the effects of the geographic pre-distribution. Then after a similar decrease of Awarenss Qulity to aroun $80 \%$ we see a much longer recovery period, which is effectively a middle ground between the performance of temporal and geographical pre-distribution. Temporal pre-distribution is effectively not active outside the 20 beacon period before the coordinated pseudonym change. This means that the slower recovers time of the mixed scheme is mostly due to the effects of the geographical pre-distribution technique. 


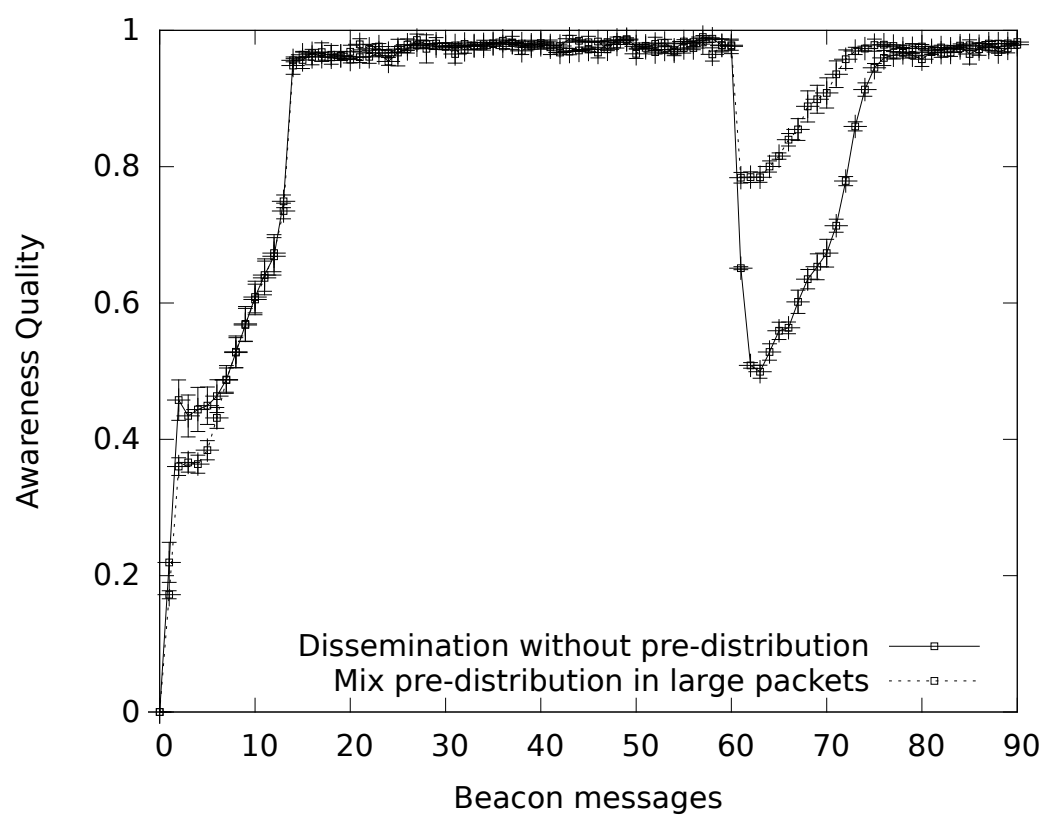

Figure 14.5: Awareness quality without and with mix pre-distribution

However, one additional small difference is visible as a small plateau between beacon perdiod 61 and 63 . The mixed scheme temporal predistribution ahead of the coordinated pseudonym changes, which announced a large number of new vehicle certificates. The receiving vehicles assume that the vehicles that used these certificate should be within a two-hop neighborhood and consequently add them to the neighbor table of vehicles in the vicinity. However, in the case of temporal pre-distribution this causes over reporting by a factor of two, which under the $\mathrm{CbCO}$ scheme leads to an erroneous assumption of more congestion in the channel and consequently more omission than necessary. This unintended increase in omissions slows down the recovery phase during the first 3 beacon periods. The beacon lifetime is calibrated at 2 beacon periods, which means that the over reporting by a factor of 2 is purged after 3 beacon cycles. This special effect was absent, compared to not performing pre-distribution, in the case of purely geographical pre-distribution and was not visible in the case of pure temporal pre-distribution.

\subsection{SUMMARY AND FUTURE WORK}

The investigations that were performed in this initial assessment of the concept of pre-distribution cover only a small subset of the solution space. As a start, we limited our simulations of single-hope dissem- 
ination and selection of pre-distribution certificates with the aim of achieving geographical effects, due to the placement and mobility of vehicles, and temporal effects, by pre-distributing certificates that will be used in the near future.

The results of this assessment show that pre-distribution can have great benefits to mitigate service degradation in the specific case of coordinated pseudonym changes. Temporal pre-distribution reduced cryptographic packet loss to a time window of 4 beacon cycles. Without temporal pre-distribution it takes between 15 and 25 beacon cycles to achieve the same recovery of application level service quality, as measured by the Awareness Quality metric. It appears highly advisable to consider this technique if coordinated pseudonym changes will be performed in moving traffic.

However, our results also show that diminishing returns make it more difficult to achieve positive results for the more generic case of geographic pre-distribution. The basic motivation of pre-distribution is to use spare bandwidth in low congestion situations in order to achieve better service quality in future circumstances. The delta between these two situations was not large enough in our investigations to show any significant effects. The increased communication load of attaching pre-distribution certificates can however lead to an increase in network packet loss effects, independent of any actual benefits. In this sense our simulations showed a negative result for the utility of geographic pre-distribution.

As future work it would be interesting to cover more of the solution space for pre-distribution techniques and to investigate pre-distribution in larger scenarios. A consideration of multi-hop pre-distribution techniques would be valuable, although the trade-off between unnecessary causing additional communication load and achieving benefits for overall service quality will be even more delicate. One solution to reduce the cost of pre-distribution could be to reduce the amount of pre-distribution. Pre-distribution could only get active when a benefit is very likely to be achieved. Heuristics for the detection of such circumstances would have to be developed. Additionally, a more careful selection of pre-distribution certificates could be helpful to increase positive effects of pre-distribution, for example based on the trajectories of vehicles. 



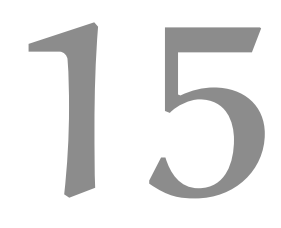

\section{SUMMARY AND FUTURE WORK}

We began this Part with the goal of finding useful ways to optimize the bandwidth consumption of secure messaging in the wireless vehicular communication channel by making the exchange of certificate material more efficient. Certificate Omission has previously been proposed to reduce the communication overhead that is introduced by securing messages. This overhead is much larger for the certificate material, which is at least 140 bytes or more, than it is for the digital signatures of the payloads. And certificate transmission is essentially redundant after the first successful delivery of a vehicles certificate.

In Chapter 11 we discussed the context and assumptions about the system model where certificate omission is performed to reduce the bandwidth consumption of secure vehicular communication. Since no large scale deployments of vehicular communication technology is available, we focus our attention on simulation studies with large numbers of vehicles. We selected to use a scenario that is in common with works of previous certificate omission studies and investigate high congestion situations, which are expected to benefit the most from bandwidth optimizations. A simpliifed analytical model was created as a reference to confirm the plausibility of our simulation studies.

The existing proposals for Certificate Omissions schemes aimed at either introducing omissions at static periods or based on neighbor events. The first approach makes it easy to reason about the statistical impact of certificate omissions on certificate delivery and ensures struct bounds on the omission of certificates. Scheduling omissions based on static periods is a conservative approach that achieves considerable bandwidth savings while keeping negative effect limited. This however limits the potential benefits of certificate omissions, since more omission could be justifiable and more bandwidth could be saved.

An omission strategy based on neighbor events aims to maximize omission by reducing the inclusion of certificate to the absolute minimum. This is essentially the opposite approach, which make the optimistic assumptions that the initial discovery of a new neighboring vehicle triggers a successful exchange of certificate material. Since this event occurs when two vehicles first come into communication range, 
it is likely that the vehicles are still separated by a large geographical distance. This implies that there is a considerable likelihood that the delivery of this event triggered certificate exchange fails, which can potentially cause a large period of unverifiable packets. We refer to unverifiable packets as cryptographic packet loss.

In Chapter 12 we proposed a novel certificate omission strategy, which aims to not optimize for maximum omissions, but instead aims to maximize service quality. For this purposed we propose to adaptively vary a certificate omission period, based on the congestion level in the communication channel. This is a better balance of the positive and negative effects of certificate omission: While a communication channel is overloaded, it is useful and important to save bandwidth through certificate omission to enable successful packet delivery for more vehicles. While a channel is almost idle, there is no motivation to use certificate omission at all. Consequently, without a justification to perform certificate omission, there is no motivation to take any risk of creating cryptographic packet loss.

The get an application level view of the impact of the different certificate omission strategies we employed Awareness Quality as a high level metric in Chapter 13. Awareness Quality turned out to be highly useful not just to judge abstract service quality, but also to investigate detailed smaller effects of each strategies. This enabled a comparison of multiple small variations of the Congestion-based Certificate Omission technique. We identified the $\mathrm{CbCO}$ scheme with a quadratic adaptation curve to have the most desirable overall performance characteristics.

Finally, in Chapter 14 we explored a novel approach to reduce the occurrence of cryptographic packet loss. After all vehicles in communication range have successfully exchanged their corresponding certificates, there is in fact little motivation to continuously exchange the same redundant certificates. While the Neighbor-based Certificate Omission scheme aimed at absolutely minimizing bandwidth consumption we explored the idea of using spare bandwidth for other beneficial purposed. Pre-distribution of certificate material using spare bandwidth could help to further minimize cryptographic packet loss. We identified two utility classes, which could potentially be achieved through pre-distribution of certificates: Pre-distribution of certificates of vehicles that are not in communication range, but will be in communication range soon, due to geographic mobility of the vehicles. And pre-distribution of certificates the will soon be useful within the communication range, due to upcoming pseudonym changes.

An initial assessment in simulation showed that geographic effects over one single hop are weak. More intelligent certificate selection could improve the utility of geographic pre-distribution, as could multihop dissemination techniques and simply limiting pre-distribution to situation where a benefit is clearly achievable. Diminishing returns 
will require careful analysis of potential applications of this technique. However, pre-distribution of certificates with the goal of preparing a coordinated certificate exchange has shown the be highly effective at reducing cryptographic packet loss. The risk of creating unverifiable packets as part of a coordinated pseudonym exchange was drastically reduced. Temporal pre-distribution of certificates can be an effective method to make pseudonym changes safe even while vehicles participate in moving traffic.

The finite resources of the wireless communication channel for $\mathrm{V}_{2} \mathrm{~V}$ communication will always be a natural limitation. Any finite resources need to be managed carefully, especially in an infrastructure that is expected to enable new safety-of-life applications. Security cannot be optional in an environment that potentially affect the safety of human life. However, security does not come free of costs. We proposed novel methods for the dissemination of cryptographic in the context of secure $\mathrm{V} 2 \mathrm{~V}$ communication to reduce these costs with respect to bandwidth consumption. The next major step in the evolution of these protocol should involve evaluations in field operational trials. However, no existing test bed is large enough to to allow studies of effects under very high communication load. Such investigations are left as future work.

The pre-distribution technique in particular is at an early evolutionary stage. Our initial assessments in simulations indicated a potential to deliver great benefits in very specific scenarios, such as coordinated pseudonym changes. Further investigations, especially involving multihop dissemination strategies, should aim to identify and formalize the conditions of significant utility. Diminishing returns will require diligence and might make it necessary to wait for the arrival of larger $\mathrm{V}_{2} \mathrm{~V}$ deployments to confirm that positive effects can truly be achieved in reality. Similarly, the related works in active certificate requests are likely to see diminishing returns due to the creation of additional load in the communication channel. A comparison with our proposal and hybrid proposals, such as the recent ETSI dissemination rules for CAM, could be further interesting work for the future. 

Part V

CONCLUSION AND FUTURE WORK 



\section{6}

SUMMARY OF CONTRIBUTIONS

This dissertation covered the topic of scalable broadcast authentication in vehicle-to-vehicle networking in three distinct ways. The aim of each approach was to find answers to a set of research questions, which have been introduced in Chapter 1.3. The first set of questions concerned a reassessment of the requirements for secure broadcast authentication in this specific application context.

Previous work has already recorded abstract requirements for security in vehicular communication, which encompasses the core $\mathrm{V}_{2} \mathrm{~V}$ broadcast communication patterns. In [113] and [114] we find classic discussion of abstract requirements such as Authentication, Availability, Non-repudiation, Privacy, Verification of data consistency, along with Real-time constraints. In chapter 3.I we arrive at essentially the same results with (Broadcast) Authenticity, (Short-term) Linkability, Non-repudiation, and Availability as the core requirements. Having answered question R 1 in the affirmative, we go further in our analysis in two directions:

Privacy as an unqualified goal of avoiding surveillance turns out to be both practically unattainable and actually counter to the whole idea of cooperative driving. The essence of cooperative driving is to enable all vehicles in the immediate vicinity to accurately track all other vehicle movements. Fundamental beaconing services, such as the CAM and BSM services in the ETSI and IEEE standards, seek to actively enable accurate tracking, to the point where identity-obscuring privacy-protection features are rendered pointless [9]. Even some kind of confidentiality of position beacons, for example in a state of a constant cryptographic mix zone [52], would allow observers to track vehicles, since this is the actual purpose of the payload material of position beacons. Previous research has suggested pseudonym change protools as an adequate countermeasure against long term tracking of vehicles [56, 97], while still permitting short term tracking. We consider the perspective of the attacker to gain a better understanding of achievable privacy in $\mathrm{V} 2 \mathrm{~V}$ communication. We observe that attempts to protect against tracking by a global all-seeing observer or by local observers in communication range is essentially futile. Observation 
coverage is the core attribute that defines attacker uncertainty, not the time between or the duration of pseudonym changes. This observation is not entirely novel, as for example the original mix zone proposal already identified attacker uncertainty as a core metric of privacy protection. However, the approach of creating attacker uncertainty by obscuring the pseudonym change process though silent zones is not acceptable for the vehicular context with implications for safety-of-life applications. The observation that only gaps in coverage create tracking uncertainty should instead inform the efforts to select pseudonym change strategies [108] in a different way. Realizing the futility of obscuring pseudonym changes against local observers makes synchronized pseudonym change strategies acceptable. The selection of pseudonym change strategies is still an undecided problem, but we see that refinements of security goals provide useful information for the solution space of problems related to broadcast authentication in $\mathrm{V}_{2} \mathrm{~V}$ communication, as suggested in question $\mathrm{R} 2$.

The other contribution of chapter 3.I is a quantification of performance constraints and communication overhead. Both attributes are highly relevant as cornerstones for actual deployments of $\mathrm{V} 2 \mathrm{~V}$ communication strategies. Our analysis of the constraints in communication overhead is founded on a survey of relevant networking constraints and proposals of interoperable communication protocols, identifying a limit of 412 bytes for cryptographic material. This limit essentially makes everything except elliptic curve based cryptographic primitives impractical for the implementation of signatures and certificate material in vehicular communication. Our quantification of performance requirements is founded on expectations of application requirements and simulations of actual communication channel capacity to derive permissible processing times of secure messages. We identify limits of 1oms latency for signature creation and a bound of 1000 signature verifications per second as the edge-cases of practical performance requirements for security overhead in scalable $\mathrm{V}_{2} \mathrm{~V}$ broadcast authentication. These values influence not only the choice of cryptographic primitives, but also the type and structure of the hardware that is required to provide adequate processing capabilities. The results are directly applicable to the discussion of hardware-assisted solutions for scalable broadcast authentication in question $\mathrm{H}_{2}$, which again confirms the suggestion of question $\mathrm{R}_{2}$ that refinements of requirements provide useful contributions for evaluating the solution space of broadcast authentication challenges.

One more objective of our reassessment of requirements was to investigate the limitations of broadcast authentication as a guarantee of security and trustworthiness of cooperative intelligent transportation systems. In chapter 4.I we presented a data lifecycle analysis to systematically look at attack surfaces of the overall cITS architecture. While 
cryptographic authentication of data in transit is a valuable security feature, it is not the only important attack vector. Attacks through sensor confusion can be cheaper and easier to implement and can be as effective as injecting manipulated data in the communication channel. Examples of this can be attacks as simple as "blinding attacks" with laser pointers against camera based systems. Also physical access as an attack vector has a large impact on the security of vehicular security. We use the description of an "evil mechanic", related to the "evil maid" attack against trust in computer hardware, to describe this attack scenario. Returning to the original question $\mathrm{R}_{3}$, about realistic attacker models, we can state that the data-centric perspective on security reveals that the realm of security for connected vehicles is certainly much larger than protection of data in transit through cryptographic means. We have proposed to analyze attack vectors and attacker roles that have not previously received serious attention in the literature.

Hardware-assisted solutions for scalable V2V broadcast authentication have been investigated in two directions in this dissertation. Chapter 7 presented a complete solution for a secure storage system of large amounts of key material, backed by a hardware-based Physically Unclonable Function (PUF). The effectiveness of this solution was shown by following several approaches, based on single-challenge and multi-challenge PUF designs. In parallel to our PUF proposal we also investigated classic secure storage designs and applied the same key derivation techniques to these as well. The result is a selection of designs that allows secure storage of private key material in a PUF and in classical secure storage. This answers question $\mathrm{H}_{1}$ with an affirmation, that PUF technology can play a useful role in scalable secure storage of key material for $\mathrm{V} 2 \mathrm{~V}$ broadcast authentication.

Research question $\mathrm{H}_{2}$ is the most applied research question of this work. A collection of on-board units from field operational test projects for vehicular communication was used to test performance aspects of cryptographic services. An extensive set of signature creation and verification benchmarks provided insight into the capabilities of contemporary cryptographic implementations of asymmetric key cryptography over elliptic curves. With the performance requirements derived from research question $\mathrm{R}_{2}$ it becomes clear that the current generation of on-board units is not capable of guaranteeing availability of cryptographic services under high loads of secure messages. In particular the requirement to provide enough computational capacity to verify up to 1000 incoming messages per second is challenging. Desktop-class computer architectures, which will eventually technologically trickle down to embedded devices such as on-board units, could provide enough computational power to fulfill the requirements of maximum load in the communication channel. An alternative to desktop class processor architectures could be hardware security modules with dedi- 
cated acceleration of cryptographic algorithms. A further investigation of such designs revealed that even for dedicated hardware designs it is challenging to achieve the benchmark of 1000 signatures per second. Only a design with massively wide multiplier construction or designs with parallel processing capabilities could provide this level of performance. However, for the current generation of on-board units it is a matter of necessity to have a hardware solution for accelerated execution of cryptographic algorithms.

Finally, we have a set of research questions centered around certificate management and enhancing scalability of $\mathrm{V} 2 \mathrm{~V}$ broadcast by reducing congestion in the communication channel. We performed multiple simulation studies of certificate omission schemes and utilized different approaches to analyze the trade-offs of certificate omission. An initial contribution was the demonstration that certificate omission can really only achieve any benefits at all when the communication channel is congested. Without congestion in the communication channel, there is nothing to gain in terms of reduction of packet loss. This has became clear already in a set of analytical considerations in chapter 11 , and was confirmed in later simulation studies through comparisons of scenarios under heavy communication load with scenarios without serious congestion in the communication channel. A second contribution is the categorization of unverifiable packets as cryptographic packet loss. This categorization allowed us to directly combine the negative consequences of certificate omission with the positive consequences into one unified packet delivery metric, while still being able to reason about the impacts of the two sources of packet loss. Answering question $C_{1}$, we identify these two aspects as the guiding properties for the trade-off between positive and negative consequences of certificate omission: The actual ability to achieve a reduction of channel congestion and the minimization of cryptographic packet loss. Our simulation results continue a line of previous simulation studies on certificate omissions, which were performed in the context of proposals of novel certificate omission schemes. As a study into scalability aspects we focus on edge cases of certificate omission performance in scenarios with high communication load. This is also useful when considering that certificate omission is unlikely to achieve a large increase of packet delivery success if no congestion exists in the communication channel. This is in fact a weakness of existing static and event-based certificate omission schemes and also answers research question $C_{3}$. These schemes perform certificate omissions even if the chance of achieving a reduction of packet loss is very small. However, performing certificate omission in such a scenario needlessly introduces a new source of packet loss, in the form of unverifiable messages. Simply maximizing omission is not the most effective certificate omission strategy. 
A more severe problem was demonstrated with the purely eventbased certificate omission scheme, which exhibited a *reduced* number of omissions under very high load. This reduction of omissions is observed during situations with many unknown neighbors and high rates of packet-loss in a congested communication channel. However, a useful omission scheme should perform the highest amount of omission when a communication channel is most congested. This sub-optimal behavior, under load, with many new communication partners and the needless introduction of certificate omission when the impact of a reduction of network packet-loss is minimal represent undesirable behavior in existing certificate omission schemes.

A novel congestion-based certificate omission scheme was presented in chapter12. This scheme utilizes a dynamic adaptation function to context-sensitively adjust the omission rate. The scheme was subsequently evaluated in multiple simulation studies, investigating the performance of packet-loss reduction with respect to both the introduction of cryptographic packet-loss and network packet-loss avoidance. The results indicate that the effectiveness of reducing network packetloss equals the most aggressive configurations of periodic omission schemes while avoiding unnecessary introduction of cryptographic packet-loss. This is particularly apparent under the most taxing scenario of encountering a large number of previously unknown vehicles. Applying the application level metric of Awareness Quality (AQL) in chapter 13 allowed us to observe the exact behavior of the investigated certificate omission schemes during the early beacon periods under such conditions. AQL visualized the speed and characteristic graphs of each certificate omission scheme during the recovery from such a scenario. The performance of congestion-based certificate omissions showed excellent performance in all situations, ranging from the least-congested to the most-taxing scenarios, leading to the answer to question $\mathrm{C}_{4}$ that congestion-based certificate omission can deliver better performance that previous certificate omission schemes. 



\section{7}

FUTURE RESEARCH DIRECTIONS

Our outlook towards future research directions will begin with the answer to the final research question $\mathrm{C}_{5}$ about the potential of predistribution as a valuable certificate management technique. Chapter 14 presented a simulation study that investigated pre-distribution with respect to two potential sources of advantageous certificate dissemination: Geographical pre-distribution and temporal pre-distribution. These two utility classes expect to derive benefits from the mobility of vehicles and from pre-announcements of upcoming certificate changes.

Our simulations demonstrated that geographic pre-distribution had only a negligible effect. Further refinements are possible, for example the selection of pre-distribution certificate could be optimized based on vehicle trajectories, or multi-hop dissemination techniques could be used in future work to enlarge the range of pre-distribution. But it is likely that diminishing returns will make it difficult to achieve noticeable benefits in exchange for introducing further complexity in the management of $\mathrm{V}_{2} \mathrm{~V}$ distribution of certificates.

On the other hand, temporal pre-distribution was shown to be very effective at eliminating a temporal increase in unverifiable packets due to the change of certificate material. In this regard we can give a positive answer to question $\mathrm{C}_{5}$.

Whether we will actually see certificate changes happening on the road at all is still an open question. As mentioned in the discussions of achievable privacy goals, the question of how to perform pseudonym changes is still an undecided problem. Multiple proposals exist, as described in [108]. The work in this dissertation indicates that synchronized pseudonym changes are acceptable from a privacy perspective and announcing the intent of changing pseudonym in combination with pre-distribution of the new pseudonym can avoid unverifiable packets. This avoidance of unverifiable packets renders such synchronized pseudonym changes safe, even while driving in moving traffic. As the initial deployments of $\mathrm{V} 2 \mathrm{~V}$ communication systems are approaching it will be necessary to select a pseudonym change strategy soon. This would ideally supported by further research into the effectiveness, safety, and efficiency of pseudonym change strategies. 
The arrival of $\mathrm{V}_{2} \mathrm{~V}$ communication in larger deployments and ultimately in the public market is also expected to enable a new generation of research efforts that can collect real-world data to measure the effect and behaviors of $\mathrm{V} 2 \mathrm{~V}$ communication in practice. Most research into complex $\mathrm{V} 2 \mathrm{~V}$ communication solutions is based on studies of simulation models or even simpler analytical considerations. The evaluation of real deployments might reveal unexpected behaviors and new research questions that have not yet been apparent.

In the domain of certificate omission protocols we observe a recent emergence of additional hybrid omission schemes. The proposal of congestion-based certificate omission in this work adaptively combines multiple variations of periodic omissions. A variation of the eventoriented neighbor-based certificate omission protocol was adopted in the ETSI set of vehicular communication standards, which combined this approach with a fallback to periodic certificate inclusion. Another recent proposal combines certificate omission with explicit requests for unknown certificates. It would be interesting in future work to evaluate certificate management schemes in actual $\mathrm{V}_{2} \mathrm{~V}$ deployments.

The domain of hardware-assisted scalability of broadcast authentication was investigated in this work with respect to an application of PUFs for secure storage and the evaluation of cryptographic performance on contemporary hardware platforms. Future research in PUF applications is hard to predict and largely orthogonal to vehicular communication applications. Since $\mathrm{V}_{2} \mathrm{~V}$ communication is a form of machine-to-machine communication there will continue to be a need to securely store key material. In the automotive domain, the concrete implementation is likely to depend more on cost-effectiveness aspects than on abstract comparative differences of tamper-resistance. A more immediate question is the future of dedicated hardware security modules. The investigations in chapter 8 showed that contemporary on-board units require hardware acceleration to achieve adequate worst-case performance. However, modern CPU designs start to subsume the feature set of hardware security modules into the native instruction set architecture. Even the roadmaps for embedded processors feature trusted execution environments, which are isolated from a generalpurpose section of a processor's execution context. Native instructions for cryptographic operations and wider register lengths within the CPU could provide sufficient performance in future.

Finally, the biggest topic for future research that became evident as a result of the studies in this work, is the identification of necessary security efforts beyond the protection of data in transit. Broadcast authentication is a fine security solution to provide cryptographic guarantees for the authenticity and trustworthiness of transmitted data. However, the real world is likely to revolve around the weakest link in the data security life cycle. Powerful attacker models with 
physical access to a vehicle or with the ability to influence sensor reading will demand new views on security as a robustness and safety topic. Questions of fail-safe and fail-secure behavior, as well as novel problems during the move from driver assistance systems to driverless vehicles, represent exciting new areas for security research in the upcoming years. 

The last few years have been a remarkable journey and a complete reboot of my life. Thank you Frank for being my supervisor and promotor. And thank you for encouraging me to return to academia and making all of this possible. I will never know what would have happened otherwise, but I am incredibly happy and thankful for the life I am having now.

Among the greatest blessings of my time in Twente was meeting Jonathan. In him I found a great colleague and an excellent supervisor. Thank you for sharing an office, for the trust, and for the support. And most of all, thank you for becoming a true friend.

I am grateful for having an impressive set of experts in my graduation committee. Thank you Pieter Hartel, Aiko Pras, Sandro Etalle, Falko Dressler, Robert Schmidt, and Peter Apers. And extra thanks to my paranimfs Elmer and Marco.

Frank recruited me to work in the DIES group of Pieter Hartel, which was later merged into the SCS group of Roel Wieringa. In both groups I always felt welcome and I want to thank all the staff and colleagues for the wonderful times we had. Thank you Bertine, Suse, Geert-Jan, and Michele. Thank you Maarten, Emmanuele, Andreas, Lorena, Damiano, Thomas and Alexandr. Thank you Stefan, Christoph, Arjan, Dina, Belgül, Eelco, Eleftheria, Peter, Jan-Willem, Riccardo and Ali. Bonus greetings shall go to the DACS group, especially to Martijn, Wouter and Geert, who shared research interests in vehicular communication and were always willing to collaborate.

One of the most extraordinary things during my time in Twente was not just doing my own research, but doing research in collaboration with students. From thesis supervision to just hanging around and hacking on projects, these experiences were among the most rewarding ones I had. Thank you Ties, Djurre, Bas, Rolf, Daan, Rens and Christina. And thanks to Peter Peters in the ICTS group for great sportsmanship and support.

I was very lucky to conduct my research efforts in the context of the European $\mathrm{FP}_{7}$ research project PRESERVE. Many thanks to all the members, organizers and supporters of this amazing project. Special thanks in particular shall go to the fearless participants of several integration meetings. Applied research is most exciting during those intense days of meeting at a test site and putting all the pieces of a research puzzle together. Thank you Michel and Christophe. Thank you Mirko, Dado, Daniel and Martin. Thank you Marcello, Stelios, Sakis and Panos. Thank you Carsten, Norbert, Sebastian and Kpatcha. 
Thank you Rim, Truyen and Brigitte. And special thanks to Andrea in Sophia Antipolis. It was a joy to work there.

Extra thanks shall also go to my new colleagues at ZF TRW, who have been watching me finish my dissertation for quite a while. Thank you Martin, Ian and Brian.

I also want to thank the new friends that I found during the last few years. No serious work could be done to completion, if it was not for the generous support of friends. Just a little bit of distraction and happiness can carry us such a long way. Thank you Nick, Dave and TkkrLab in Enschede. Thank you Qin, Jorn, Cindy and Ravi. It was a joy to share time together and I hope we will stay in touch.

Finally, I want to thank my family. You have always been supportive, always been there, and I know that you will always be. Thank you Sonja and Peter, mom and dad. Every one of my achievements is a success thanks to you. Thank you also to my siblings Martina and Christian for the special kind of love that only siblings have for each other. And thanks to your partners Manuel and Kathi. And thank you also to the new members of my extended family, Fatinha, Sofia and Daniel. You are lovely people, who welcomed me with such open arms. This finally leads me to thank the most important person I met during the journey of these past years. My dear Tânia. I will forever be grateful for the events that led us both to find each other. It is now unimaginable how life could be without you, without your affection and support. Thank you for everything. For continuously exploring what might make us happy. For your unwavering support. For your love. For becoming my wife.

Here's to the next adventures. Thank you all.

Michael Feiri

Solihull, September 2016 
[1] F Ahmed-Zaid, F Bai, S Bai, C Basnayake, B Bellur, S Brovold, G Brown, L Caminiti, D Cunningham, H Elzein, et al. Vehicle Safety Communications-Applications (VSC-A) Final Report: Appendix Volume 3 Security. Tech. rep. 2011.

[2] Ludovic Apvrille, Rachid El Khayari, Olaf Henniger, Yves Roudier, Hendrik Schweppe, Hervé Seudié, Benjamin Weyl, and Marko Wolf. "Secure automotive on-board electronics network architecture." In: World Automotive Congress (FISITA '10) (2010).

[3] Elaine B Barker and John Michael Kelsey. Recommendation for random number generation using deterministic random bit generators (revised). US Department of Commerce, Technology Administration, National Institute of Standards and Technology, Computer Security Division, Information Technology Laboratory, 2007.

[4] Rimon Barr, Zygmunt J. Haas, and Robbert van Renesse. "Scalable Wireless Ad hoc Network Simulation." In: Handbook on Theoretical and Algorithmic Aspects of Sensor, Ad Hoc Wireless, and Peer-to-Peer Networks. CRC Press, Aug. 2005. Chap. 19, pp. 297-311.

[5] Lejla Batina, Sıddıka Berna Örs, Bart Preneel, and Joos Vandewalle. "Hardware architectures for public key cryptography." In: Integration, the VLSI journal 34.1 (2003), pp. 1-64.

[6] A.R. Beresford and F. Stajano. "Location privacy in pervasive computing." In: Pervasive Computing, IEEE 2.1 (2003), pp. 46-55. ISSN: 1536-1268. DOI: 10 . 1109 / MPRV . 2003. 1186725.

[7] Daniel J Bernstein. “Curve25519: new Diffie-Hellman speed records." In: Public Key Cryptography-PKC 2006. Springer, 2006, pp. 207-228. 
[8] Daniel J. Bernstein and Tanja Lange. "Analysis and optimization of elliptic-curve single-scalar multiplication." In: IACR Cryptology ePrint Archive 2007 (2007), p. 455.

[9] N. Bismeyer, S. Mauthofer, K.M. Bayarou, and F. Kargl. "Assessment of node trustworthiness in VANETs using data plausibility checks with particle filters." In: Vehicular Networking Conference. IEEE, 2012, pp. 78-85. DOI: 10 . 1109/VNC . 2012.6407448.

[10] Norbert Bissmeyer, Hagen Stubing, Elmar Schoch, Stefan Gotz, Jan Peter Stotz, and Brigitte Lonc. "A generic public key infrastructure for securing Car-to-X communication." In: 18th ITS World Congress. 2011.

[11] Sebastian Bittl, Berke Aydinli, and Karsten Roscher. "Communication Technologies for Vehicles: 8th International Workshop, Nets4Cars/Nets4Trains/Nets4Aircraft 2015, Sousse, Tunisia, May 6-8, 2015. Proceedings." In: ed. by Mohamed Kassab, Marion Berbineau, Alexey Vinel, Magnus Jonsson, Fabien Garcia, and José Soler. Cham: Springer International Publishing, 2015. Chap. Effective Certificate Distribution in ETSI ITS VANETs Using Implicit and Explicit Requests, pp. 72-83. ISBN: 978-3-319-17765-6. DOI: 10. 1007/978- 3-319-17765-6_7.

[12] Sebastian Bittl, Berke Aydinli, and Karsten Roscher. "Distribution of Pseudonym Certificates via Bursts for VANETs with Low and Medium Mobility." In: 2015 8th IFIP Wireless and Mobile Networking Conference (WMNC). IEEE. 2015, pp. 227-230.

[13] Norbert Bißmeyer et al. PRESERVE D1.3 V2X Security Architecture v2. Deliverable. PRESERVE consortium, 2014.

[14] Mate Boban and Pedro M d'Orey. "Measurement-based evaluation of cooperative awareness for $\mathrm{V}_{2} \mathrm{~V}$ and $\mathrm{V}_{2} \mathrm{I}$ communication." In: Vehicular Networking Conference (VNC), 2014 IEEE. IEEE. 2014, pp. 1-8.

[15] Dan Boneh, Glenn Durfee, and Matt Franklin. "Lower bounds for multicast message authentication." In: $A d-$ vances in Cryptology-EUROCRYPT 2001. Springer, 2001, pp. 437-452. 
[16] Daniel RL Brown, Robert Gallant, and Scott A Vanstone. "Provably secure implicit certificate schemes." In: Financial Cryptography. Springer. 2002, pp. 156-165.

[17] G. Calandriello, P. Papadimitratos, J.-P. Hubaux, and A. Lioy. "On the Performance of Secure Vehicular Communication Systems." In: Dependable and Secure Computing, IEEE Transactions on 8.6 (2011), pp. 898 -912. ISSN: 15455971. DOI: 10.1109/TDSC . 2010.58.

[18] G. Calandriello, P. Papadimitratos, J-P Hubaux, and Antonio Lioy. "On the Performance of Secure Vehicular Communication Systems." In: IEEE Trans. Dependable Sec. Comput. 8.6 (2011), pp. 898-912. ISSN: 1545-5971. DOI: 10.1109/ TDSC . 2010. 58.

[19] Giorgio Calandriello, Panos Papadimitratos, Jean-Pierre Hubaux, and Antonio Lioy. "Efficient and robust pseudonymous authentication in VANET." In: Proceedings of the fourth ACM international workshop on Vehicular ad hoc networks. VANET '07. Montreal, Quebec, Canada: ACM, 2007, pp. 19-28. ISBN: 978-1-59593-739-1.

[20] Jan Camenisch and Els van Herreweghen. "Design and implementation of the idemix anonymous credential system." In: Proceedings of 9th Conference on Computer and Communications Security. ACM. 2002, pp. 21-30.

[21] Claudia Campolo and Antonella Molinaro. "Multichannel communications in vehicular ad hoc networks: a survey." In: IEEE Communications Magazine 51.5 (2013), pp. 158169.

[22] Lidong Chen. SP 800-56C. Recommendation for Key Derivation through Extraction-then-Expansion. Tech. rep. Gaithersburg, MD, United States, 2011.

[23] Nanxi Chen, Mario Gerla, Dijiang Huang, and Xiaoyan Hong. "Secure, selective group broadcast in vehicular networks using dynamic attribute based encryption." In: 9th IFIP Annual Mediterranean Ad Hoc Networking Workshop (Med-Hoc-Net '10) (2010), pp. 1-8. 
[24] Qingqing Chen, György Csaba, Paolo Lugli, Ulf Schlichtmann, and Ulrich Rührmair. "The Bistable Ring PUF: A new architecture for strong Physical Unclonable Functions." In: IEEE International Symposium on Hardware-Oriented Security and Trust (HOST '11) (2011), pp. 134-141.

[25] Tat Wing Chim, Siu-Ming Yiu, Lucas CK Hui, and Victor OK Li. "SPECS: Secure and privacy enhancing communications schemes for VANETs." In: Ad Hoc Networks 9.2 (2011), pp. 189-203.

[26] David R. Choffnes and Fabián E. Bustamante. "An integrated mobility and traffic model for vehicular wireless networks." In: Proceedings of the 2nd ACM international workshop on Vehicular ad hoc networks. VANET '05. Cologne, Germany: ACM, 2005, pp. 69-78. ISBN: 1-59593-141-4. DOI: 10.1145/1080754. 1080765.

[27] Stacy C Davis, Susan W Diegel, and Robert G Boundy. “Transportation energy data book." In: (2008).

[28] Y. Dodis, M. Reyzin, and A. Smith. "Fuzzy Extractors: How to generate strong keys from biometrics and other noisy data." In: Advances in Cryptology - EUROCRYPT 2004. Vol. 3027. LNCS. 2004, pp. 523-540.

[29] Guerric Meurice de Dormale and Jean-Jacques Quisquater. "High-speed hardware implementations of elliptic curve cryptography: A survey." In: Journal of systems architecture 53.2 (2007), pp. 72-84.

[30] II ECRYPT. Yearly Report on Algorithms and Keysizes (2012). D. SPA. 20 Rev. 1.o. Tech. rep. ICT-2007-216676 ECRYPT II, 2012.

[31] ETSI TC ITS. ETSI TS 102638 V1.1.1 - Intelligent Transport Systems (ITS); Vehicular Communications; Basic Set of Applications; Definitions. Standard. TC ITS, 2009.

[32] ETSI TC ITS. ETSI TS 102731 V1.1.1 - Intelligent Transport Systems (ITS); Security; Security Services and Architecture. Standard. TC ITS, 2010.

[33] ETSI TC ITS. ETSI TS 102867 V1.1.1 - Intelligent Transport Systems (ITS); Security; Stage 3 mapping for IEEE 1609.2. Standard. TC ITS, 2012. 
[34] ETSI TC ITS. ETSI TS 102941 V1.1.1 - Intelligent Transport Systems (ITS); Security; Trust and Privacy Management. Standard. TC ITS, 2012.

[35] ETSI TC ITS. ETSI TS 103097 V1.1.1 - Intelligent Transport Systems (ITS); Security; Security Header and Certificate Formats. Standard. TC ITS, 2013.

[36] ETSI. ETSI ES 202663 V1.1.o; Intelligent Transport Systems (ITS); European profile standard for the physical and medium access control layer of Intelligent Transport Systems operating in the $5 \mathrm{GHz}$ frequency band. 2009.

[37] ETSI. ETSI TS 102 636-4-1 V1.1.1; Intelligent Transport Systems (ITS); Vehicular communications; GeoNetworking; Part 4: Geographical addressing and forwarding for point-to-point and point-to-multipoint communications; Sub-part 1: MediaIndependent Functionality. 2011.

[38] ETSI. ETSI TS 102 636-6-1 V1.1.1; Intelligent Transport Systems (ITS); Vehicular Communications; GeoNetworking; Part 6: Internet Integration; Sub-part 1: Transmission of IPv6 Packets over GeoNetworking Protocols. 2011.

[39] ETSI. ETSI TS 102 636-6-1 V1.2.1; Intelligent Transport Systems (ITS); Vehicular Communications; Basic Set of Applications; Part 2: Specification of Cooperative Awareness Basic Service. 2011.

[40] ETSI. ETSI TS 102687 V1.1.1; Intelligent Transport Systems (ITS); Decentralized Congestion Control Mechanisms for Intelligent Transport Systems operating in the $5 \mathrm{GHz}$ range; Access layer part. 2011.

[41] TCITS ETSI. "ETSI TS 103097 V1.1.1-intelligent transport systems (ITS); security; security header and certificate formats." In: Standard, TC ITS (2013).

[42] Barker Elaine, Johnson Don, and Smid Miles. SP 800-56A. Recommendation for Pair-Wise Key Establishment Schemes Using Discrete Logarithm Cryptography. Tech. rep. Gaithersburg, MD, United States, 2007.

[43] Michael Elkins. MIME security with pretty good privacy (PGP). RFC 2015. 1996. 
[44] D. Wing F. Andreasen M. Baugher. "RFC 4568: Session Description Protocol (SDP) Security Descriptions for Media Streams." In: IETF, July (2006).

[45] Junfeng Fan, Kazuo Sakiyama, and Ingrid Verbauwhede. "Montgomery modular multiplication algorithm on multicore systems." In: Signal Processing Systems, 2007 IEEE Workshop on. IEEE. 2007, pp. 261-266.

[46] Federal Information Processing Standards. Digital Signature Standard (DSS) - FIPS 186-3. FIPS PUB. 2009.

[47] M. P. Feiri, J. Y. Petit, and F. Kargl. "Congestion-based Certificate Omission in VANETs." In: Ninth ACM International Workshop on Vehicular Ad Hoc Networks, VANET 2012, Low Wood Bay, Lake District, United Kingdom (2012), pp. $135-138$.

[48] M. P. Feiri, J. Y. Petit, and F. Kargl. "Evaluation of Congestionbased Certificate Omission in VANETs." In: 4th IEEE Vehicular Networking Conference (VNC '12) (2012), pp. 101108. ISSN: 2157-9865.

[49] V Fischer, A Aubert, F Bernard, B Valtchanov, JL Danger, and $\mathrm{N}$ Bochard. "True random number generators in configurable logic devices." In: Project ANR-ICteR (2009), pp. 1-58.

[5o] Viktor Fischer. "A closer look at security in random number generators design." In: Constructive Side-Channel Analysis and Secure Design. Springer, 2012, pp. 167-182.

[51] TrueCrypt Foundation. TrueCrypt - Free open-source on-thefly encryption. Retrieved July 10, 2013 from http://truecrypt.org/.

[52] Julien Freudiger, Maxim Raya, Márk Félegyházi, Panos Papadimitratos, et al. "Mix-zones for location privacy in vehicular networks." In: Proceedings of the first international workshop on wireless networking for intelligent transportation systems (Win-ITS). 2007.

[53] D. Förster, F. Kargl, and H. Löhr. "PUCA: A pseudonym scheme with user-controlled anonymity for vehicular ad-hoc networks (VANET)." In: 2014 IEEE Vehicular Networking Conference (VNC). 2014, pp. 25-32. DOI: 10.1109/VNC. 2014.7013305. 
[54] David Förster, Frank Kargl, and Hans Löhr. "PUCA: A pseudonym scheme with user-controlled anonymity for vehicular ad-hoc networks (VANET)." In: Vehicular Networking Conference (VNC), 2014 IEEE. IEEE. 2014, pp. 2532.

[55] Dominie Garcia, Andrea Waite, Richard Walsh, Blake Sheppard, Larry Frank, and Dan Jeffers. Certificate Management Entities for Connected Vehicle Environment. Public Workshop Read-Ahead Document. Technical report FHWAJPO-12-038. Research and Innovative Technology Administration, 2012.

[56] Philippe Golle, Dan Greene, and Jessica Staddon. "Detecting and correcting malicious data in VANETs." In: Proceedings of the 1st ACM international workshop on Vehicular ad hoc networks. ACM. 2004, pp. 29-37.

[57] Shay Gueron and Vlad Krasnov. "Fast prime field ellipticcurve cryptography with 256-bit primes." In: Journal of Cryptographic Engineering (2013), pp. 1-11.

[58] Jason J Haas, Yih-Chun Hu, and Kenneth P Laberteaux. "Design and analysis of a lightweight certificate revocation mechanism for VANET." In: Proceedings of the sixth ACM international workshop on VehiculAr InterNETworking. ACM. 2009, pp. 89-98.

[59] Jérôme Härri, Fethi Filali, Christian Bonnet, and Marco Fiore. "VanetMobiSim: generating realistic mobility patterns for VANETs." In: Proceedings of 3 rd International Workshop on Vehicular ad hoc networks. ACM. 2006, pp. 96-97.

[6o] Hannes Hartenstein and Kenneth Laberteaux. VANET: vehicular applications and inter-networking technologies. Vol. 1. Wiley Online Library, 2010.

[61] Erwin Hess, Bernd Meyer, and Norbert Janssen. “Design of long integer arithmetic units for public-key algorithms." In: Proc. of EUROSMART Security Conference. Citeseer. 2000, pp. 325-334.

[62] Leping Huang, Hiroshi Yamane, Kanta Matsuura, and Kaoru Sezaki. "Towards modeling wireless location privacy." In: Privacy Enhancing Technologies. Springer. 2006, pp. 59-77. 
[63] IEEE Standard for Wireless Access in Vehicular Environments - Security Services for Applications and Management Messages. IEEE Std. 1609.2-2013. 2013.

[64] IEEE. "IEEE Standard Specifications for Public-Key CryptographyAmendment 1: Additional Techniques." In: IEEE Std 1363a2004 (Amendment to IEEE Std 1363-2000) (2004), pp. 1-159. DOI: 10.1109/IEEESTD . 2004 . 94612.

[65] IEEE. IEEE 802.11p-2010 - Wireless Access in Vehicular Environments. New York, NY, USA, 2010.

[66] ISO/IEC. "Information technology - Security techniques Encryption algorithms - Part 2: Asymmetric ciphers." In: ISO/IEC 18033-2 (2006). Ed. by Victor Shoup.

[67] Iulia Ivan, Philippe Besnier, Matthieu Crussiere, Mhamed Drissi, Lois Le Danvic, Mickael Huard, and Eric Lardjane. "Physical layer performance analysis of V2V communications in high velocity context." In: Intelligent Transport Systems Telecommunications,(ITST), 2009 9th International Conference on. IEEE. 2009, pp. 409-414.

[68] Iulia Ivan, Philippe Besnier, Xavier Bunlon, and Philippe Boutier. "Measurement of V2X Prototypes Performance. comparison with simulation results." In: Antennas and Propagation (EuCAP), 2013 7th European Conference on. IEEE. 2013, pp. 1432-1435.

[69] Attila Jaeger, Norbert Bißmeyer, Hagen Stübing, and SorinA. Huss. "A Novel Framework for Efficient Mobility Data Verification in Vehicular Ad-hoc Networks." English. In: International Journal of Intelligent Transportation Systems Research 10.1 (2012), pp. 11-21. ISSN: 1348-8503. DOI: $10.1007 / \mathrm{s} 13177$ - 011-0038- 9 .

[7o] Burt Kaliski. "RFC 2898: PKCS\# 5: Password-Based Cryptography Specification Version 2.o." In: IETF, September (2000).

[71] Pandurang Kamat, Arati Baliga, and Wade Trappe. "An identity-based security framework for VANETs." In: Proceedings of 3 rd International Workshop on Vehicular ad hoc networks. ACM. 2006, pp. 94-95. 
[72] Frank Kargl and Elmar Schoch. "Simulation of MANETs: a qualitative comparison between JiST/SWANS and ns2." In: Proceedings of the 1st international workshop on System evaluation for mobile platforms. ACM. 2007, pp. 41-46.

[73] Frank Kargl, Elmar Schoch, Björn Wiedersheim, and Tim Leinmüller. "Secure and Efficient Beaconing for Vehicular Networks (Short Paper)." In: 5th ACM International Workshop on Vehicular Ad Hoc Networks (VANET 2008). San Francisco, USA: ACM, 2008. DOI: 10.1145/1410043.1410060.

[74] Stefan Katzenbeisser, Ünal Kocabaş, Vincent van der Leest, Ahmad-Reza Sadeghi, Geert-Jan Schrijen, and Christian Wachsmann. "Recyclable PUFs: Logically Reconfigurable PUFs." In: Journal of Cryptographic Engineering 1.3 (2011), pp. 177-186.

[75] John Kelsey, Bruce Schneier, Chris Hall, and David Wagner. "Secure applications of low-entropy keys." In: Information Security. Springer, 1998, pp. 121-134.

[76] Wolfgang Killmann and Werner Schindler. "A proposal for: Functionality classes for random number generators." In: Bundesamt für Sicherheit in der Informationstechnik (BSI), Bonn (2011).

[77] Bum Han Kim, Kyu Young Choi, Jun Ho Lee, and Dong Hoon Lee. "Anonymous and Traceable Communication Using Tamper-Proof Device for Vehicular Ad Hoc Networks." In: International Conference on Convergence Information Technology (2007), pp. 681-686.

[78] Stephan Kleber, Florian Unterstein, Matthias Matousek, Frank Kargl, Frank Slomka, and Matthias Hiller. Secure Execution Architecture based on PUF-driven Instruction Level Code Encryption. Cryptology ePrint Archive, Report 2015/651. 2015.

[79] Pierre Kleberger, Tomas Olovsson, and Erland Jonsson. "Security aspects of the in-vehicle network in the connected car." In: IEEE Intelligent Vehicles Symposium (IV). 2011, pp. 528-533. 
[8o] Miroslav Knezevic, Ventzislav Nikov, and Peter Rombouts. Low-Latency ECDSA Signature Verification-A Road Towards Safer Traffic. Cryptology ePrint Archive, Report 2014/862. http://eprint.iacr. org/. 2014.

[81] Werner Koch. GnuPG - The GNU Privacy Guard. Retrieved July 10, 2013 from http:/ /gnupg.org/. 2013.

[82] Hugo Krawczyk. "Cryptographic extraction and key derivation: The HKDF scheme." In: Advances in CryptologyCRYPTO 2010. Springer, 2010, pp. 631-648.

[83] Hugo Krawczyk and Pasi Eronen. HMAC-based Extractand-Expand Key Derivation Function (HKDF). RFC 5869. 2010.

[84] Tanja Lange. PUFFIN - The Physically unclonable functions found in standard PC components project. Retrieved July 10, 2013 from http://puffin.eu.org/. 2013.

[85] Stéphanie Lefèvre, Jonathan Petit, Ruzena Bajcsy, Christian Laugier, and Frank Kargl. "Impact of $\mathrm{V}_{2} \mathrm{X}$ privacy strategies on intersection collision avoidance systems." In: Vehicular Networking Conference. IEEE, 2013, pp. 71-78. DOI: 10.1109/VNC. 2013.6737592.

[86] Tim Leinmüller, Levente Buttyan, Jean-Pierre Hubaux, Frank Kargl, Rainer Kroh, Panos Papadimitratos, Maxim Raya, and Elmar Schoch. "Sevecom - Secure Vehicle Communication." In: IST Mobile and Wireless Communication Summit (2006), pp. 1-5.

[87] J.-P. M. G. Linnartz and P. Tuyls. "New Shielding Functions to Enhance Privacy and Prevent Misuse of Biometric Templates." In: Audio-and Video-Based Biometrie Person Authentication (AVBPA '03). LNCS 2688 (2003), pp. 393-402.

[88] Anna Lysyanskaya, Roberto Tamassia, and Nikos Triandopoulos. "Multicast authentication in fully adversarial networks." In: IEEE Symposium on Security and Privacy. 2004, pp. 241-253.

[89] Anna Lysyanskaya, Ronald L. Rivest, Amit Sahai, and Stefan Wolf. "Pseudonym Systems." In: 6th Annual International Workshop on Selected Areas in Cryptography (SAC '99) (1999), pp. 184-199. 
[90] Roel Maes, Anthony Herrewege, and Ingrid Verbauwhede. "PUFKY: A Fully Functional PUF-Based Cryptographic Key Generator." In: Cryptographic Hardware and Embedded Systems (CHES '12) (2012), pp. 302-319. DOI: 10.1007/9783- 642 - 33027-8_18.

[91] Francesca Martelli, M Elena Renda, and Paolo Santi. "Measuring IEEE 802.11 p performance for active safety applications in cooperative vehicular systems." In: Vehicular Technology Conference (VTC Spring), 2011 IEEE 73rd. IEEE. 2011, pp. 1-5.

[92] Jens Mittag, Felix Schmidt-Eisenlohr, Moritz Killat, Jérôme Härri, and Hannes Hartenstein. "Analysis and design of effective and low-overhead transmission power control for VANETs." In: Proceedings of the fifth ACM international workshop on VehiculAr Inter-NETworking. VANET '08. San Francisco, California, USA: ACM, 2008, pp. 39-48. IsBN: 978-1-60558-191-0. DOI: 10.1145/1410043.1410051.

[93] Sebastian Mödersheim and Paolo Modesti. "Verifying sevecom using set-based abstraction." In: Wireless Communications and Mobile Computing Conference (IWCMC), 2011 7th International. IEEE. 2011, pp. 1164-1169.

[94] Kees Moerman, Timo van Roermund, and Miroslav Knezevic. "A Realistic Approach to Message Verification in Car-to-Car Communication." In: 19th ITS World Congress (2012).

[95] Gary L Mullen, Daniel Panario, and Igor E Shparlinski. Finite Fields and Applications: Eighth International Conference on Finite Fields and Applications, July 9-13, 2007, Melbourne, Australia. Vol. 461. American Mathematical Soc., 2008.

[96] Benedikt Ostermaier, Florian Dötzer, and Markus Strassberger. "Enhancing the Security of Local Danger Warnings in VANETs - A Simulative Analysis of Voting Schemes." In: Proceedings of the 2nd International Conference on Availability, Reliability and Security (ARES). 2007, pp. 422-431.

[97] P. Papadimitratos, A. Kung, J.-P. Hubaux, and F. Kargl. "Privacy and Identity Management for Vehicular Communication Systems: A Position Paper." In: Workshop on 
Standards for Privacy in User-Centric Identity Management. Zurich, Switzerland, 2006.

[98] P. Papadimitratos, G. Calandriello, J.-P. Hubaux, and A. Lioy. "Impact of vehicular communications security on transportation safety." In: INFOCOM Workshops 2008, IEEE. 2008, pp. 1-6. DOI: 10.1109/INFOCOM. 2008. 4544663.

[99] P. Papadimitratos, G. Calandriello, J-P Hubaux, and Antonio Lioy. "Impact of vehicular communications security on transportation safety." In: INFOCOM Workshops 2008, IEEE. 2008, pp. 1-6. DOI: 10 . 1109/INF0C0M . 2008. 4544663.

[100] Panagiotis Panos Papadimitratos, Ghita Mezzour, and Jean-Pierre Hubaux. "Certificate revocation list distribution in vehicular communication systems." In: Proceedings of 5 th International Workshop on VehiculAr Inter-NETworking. ACM. 2008, pp. 86-87.

[101] R. Pappu. "Physical One-Way Functions." PhD thesis. MIT, 2001.

[102] R. Pappu, B. Recht, J. Taylor, and N. Gershenfeld. "Physical One-Way Functions." In: Science 297 (2002), pp. 20262030.

[103] Colin Percival. "Stronger Key Derivation via Sequential Memory-Hard Functions." In: The Technical BSD Conference (BSDCan 'o9) (2009).

[104] A Perrig, R Canetti, JD Tygar, and Dawn Song. "The TESLA broadcast authentication protocol." In: RSA CryptoBytes 5.2 (2002), pp. 2-13.

[105] J. Y. Petit, M. P. Feiri, and F. Kargl. "Spoofed Data Detection in VANETs using Dynamic Thresholds." In: Proceedings of the IEEE Vehicular Networking Conference (VNC 2011), Amsterdam, Netherlands (2011), pp. 25-32.

[106] J. Petit, B. Stottelaar, M. Feiri, and F. Kargl. "Self-driving cars: Don't trust your sensors." In: BlackHat Europe 2015. 2015.

[107] Jonathan Petit and Zoubir Mammeri. "Authentication and Consensus Overhead in Vehicular Ad Hoc Networks." In: Telecommunication Systems (2011), pp. 1-14. ISSN: 1018-4864. DOI: $10.1007 / \mathrm{s} 11235$ - $011-9589-y$. 
[108] Jonathan Petit, Florian Schaub, Michael Feiri, and Frank Kargl. "Pseudonym schemes in vehicular networks: A survey." In: IEEE communications surveys $\mathcal{E}$ tutorials 17.1 (2015), pp. 228-255.

[109] Rainer Plaga and Frank Koob. "A formal definition and a new security mechanism of physical unclonable functions." In: Measurement, Modelling, and Evaluation of Computing Systems and Dependability and Fault Tolerance. Springer, 2012, pp. 288-301.

[110] Niels Provos and David Mazieres. "A Future-Adaptable Password Scheme." In: USENIX Annual Technical Conference, FREENIX Track (1999), pp. 81-91.

[111] Blake Ramsdell. Secure/Multipurpose Internet Mail Extensions (S/MIME) Version 3.1 Message Specification. RFC 2633. 2004.

[112] M. Raya, P. Papadimitratos, and J.-P. Hubaux. "SECURING VEHICULAR COMMUNICATIONS." In: Wireless Communications, IEEE 13.5 (2006), pp. 8-15. ISSN: 15361284. DOI: 10.1109/WC - M. 2006. 250352.

[113] Maxim Raya and Jean-Pierre Hubaux. "The security of vehicular ad hoc networks." In: Proceedings of the 3 rd ACM workshop on Security of ad hoc and sensor networks. ACM. 2005, pp. 11-21.

[114] Maxim Raya and Jean-Pierre Hubaux. "Securing vehicular ad hoc networks." In: Journal of Computer Security 15.1 (2007), pp. 39-68.

[115] Maxim Raya, Panos Papadimitratos, and Jean-Pierre Hubaux. "Securing vehicular communications." In: IEEE Wireless Communications Magazine, Special Issue on Inter-Vehicular Communications 13.LCA-ARTICLE-2006-015 (2006), pp. 815.

[116] Dominik Reichl. KeePass Password Safe. Retrieved July 10, 2013 from http://keepass.info.

[117] Ulrich Rührmair, Frank Sehnke, Jan Sölter, Gideon Dror, Srinivas Devadas, and Jürgen Schmidhuber. "Modeling attacks on physical unclonable functions." In: 17 th ACM conference on Computer and communications security (CCS '10) (2010), pp. 237-249. DOI: 10.1145/1866307. 1866335. 
[118] Joanna Rutkowska and Alexander Tereshkin. "Evil maid goes after TrueCrypt." In: The Invisible Things Lab (Oct. 2009).

[119] SAE International. DSRC Implementation Guide - A guide to users of SAE J2735 message sets over DSRC. Tech. rep. v2o. 2010. URL: http : / / www . sae . org / standardsdev / dsrc / DSRCImplementationGuide.pdf.

[120] Kazuo Sakiyama, Elke De Mulder, Bart Preneel, and Ingrid Verbauwhede. "A parallel processing hardware architecture for elliptic curve cryptosystems." In: Acoustics, Speech and Signal Processing, 2006. ICASSP 2006 Proceedings. 2006 IEEE International Conference on. Vol. 3. IEEE. 2006, pp. III-III.

[121] Krishna Sampigethaya, Leping Huang, Mingyan Li, Radha Poovendran, K. Matsuura, and K. Sezaki. "CARAVAN: Providing Location Privacy for VANET." In: 3 rd Workshop on Embedded Security in Cars (ESCAR '05). 2005, pp. 1-15.

[122] Florian Schaub, Zhendong Ma, and Frank Kargl. "Privacy requirements in vehicular communication systems." In: Computational Science and Engineering, 2009. CSE'o9. International Conference on. Vol. 3. IEEE. 2009, pp. 139-145.

[123] Florian Schaub, Frank Kargl, Zhendong Ma, and Michael Weber. "V-tokens for Conditional Pseudonymity in VANETs." In: Wireless Communications and Networking Conference (WCNC), 2010 IEEE. IEEE. 2010, pp. 1-6.

[124] R. Schmidt, R. Lasowski, T. Leinmüller, C. Linnhoff-Popien, and G. Schafer. "An approach for selective beacon forwarding to improve cooperative awareness." In: Vehicular Networking Conference (VNC), 2010 IEEE. 2010, pp. 182188. DOI: 10.1109/VNC.2010.5698243.

[125] Robert K Schmidt and Tim Leinmüller. "A Spatio-Temporal Metric for the Evaluation of Cooperative Awareness." In: 18th World Congress on Intelligent Transport Systems. 2011.

[126] Robert K Schmidt, Achim Brakemeier, Tim Leinmüller, Frank Kargl, and Günter Schäfer. "Advanced carrier sensing to resolve local channel congestion." In: Proceedings of the Eighth ACM international workshop on Vehicular internetworking. ACM. 2011, pp. 11-20. 
[127] Bruce Schneier. Password Safe - The security of Twofish in a password database. Retrieved July 10, 2013 from http:/ /schneier.com/passsafe.html.

[128] Elmar Schoch and Frank Kargl. "On the Efficiency of Secure Beaconing in VANETs." In: 3 rd ACM Conference on Wireless Network Security (WiSec '10). 2010, pp. 111-116. ISBN: 978-1-60558-923-7. DOI: 10 . 1145/1741866 . 1741885.

[129] Elmar Schoch, Michael Feiri, Frank Kargl, and Michael Weber. "Simulation of Ad Hoc Networks: ns-2 compared to JiST/SWANS." In: First International Conference on Simulation Tools and Techniques for Communications, Networks and Systems (SimuTools 2008). Marseilles, France, Mar. 2008.

[130] Torsten Schütze. "Automotive security: Cryptography for car2x communication." In: Embedded World Conference. 2011.

[131] Túlio Cicero Salvaro de Souza, Jean Everson Martina, and Ricardo Felipe Custódio. "Audit and backup procedures for hardware security modules." In: $7^{\text {th }}$ Symposium on Identity and Trust on the Internet (IDtrust 'o8) (2008), pp. 8997. DOI: 10.1145/1373290.1373302.

[132] Graham Steel. Towards a formal security analysis of the Sevecom API. 2009.

[133] Jan Peter Stotz, Norbert Bißmeyer, Frank Kargl, Stefan Dietzel, Panos Papadimitratos, and Christian Schleiffer. PRESERVE D1.1 Security Requirements of Vehicle Security Architecture. Deliverable. PRESERVE consortium, 2011.

[134] Hagen Stubing, Attila Jaeger, Christoph Schmidt, and Sorin A Huss. "Verifying mobility data under privacy considerations in Car-to-X communication." In: Proceedings of 17th ITS World Congress. 2010.

[135] I. Studnia, V. Nicomette, E. Alata, Y. Deswarte, M. Kaaniche, and Y. Laarouchi. "Survey on security threats and protection mechanisms in embedded automotive networks." In: 43rd Annual IEEE/IFIP Conference on Dependable Systems and Networks Workshop (DSN-W). 2013, pp. 1-12. DOI: 10.1109/DSNW. 2013.6615528. 
[136] Robert Szerwinski and Tim Güneysu. "Exploiting the power of GPUs for asymmetric cryptography." In: Cryptographic Hardware and Embedded Systems-CHES 2008. Springer, 2008, pp. 79-99.

[137] E. Rescorla T. Dierks. "RFC 5246: The Transport Layer Security (TLS) Protocol Version 1.2." In: IETF, August (2008).

[138] William Whyte, André Weimerskirch, Vipin Kumar, and Thorsten Hehn. "A security credential management system for $\mathrm{V} 2 \mathrm{~V}$ communications." In: Vehicular Networking Conference (VNC), 2013 IEEE. IEEE. 2013, pp. 1-8.

[139] B. Wiedersheim, Zhendong Ma, F. Kargl, and P. Papadimitratos. "Privacy in inter-vehicular networks: Why simple pseudonym change is not enough." In: 7 th International Conference on Wireless On-demand Network Systems and Services (WONS '10) (2010). DOI: 10.1109/WONS. 2010. 5437115.

[140] "Wireless LAN Medium Access Control (MAC) and Physical Layer (PHY) Specifications." In: IEEE Std 802.11-2012 (Revision of IEEE Std 802.11-2007) (2012), pp. 1-2793. DOI: 10.1109/IEEESTD . 2012.6178212.

[141] Marko Wolf and Timo Gendrullis. "Design, Implementation, and Evaluation of a Vehicular Hardware Security Module." In: Information Security and Cryptology (ICISC). Vol. 7259. 2011, pp. 302-318. ISBN: 978-3-642-31911-2. DOI: 10 . 1007/978- 3-642-31912-9_20.

[142] Marko Wolf, André Weimerskirch, and Thomas Wollinger. "State of the art: Embedding security in vehicles." In: EURASIP Journal on Embedded Systems 2007 (2007).

[143] Lo-Yao Yeh, Yen-Cheng Chen, and Jiun-Long Huang. "ABACS: An Attribute-Based Access Control System for Emergency Services over Vehicular Ad Hoc Networks." In: IEEE Journal on Selected Areas in Communications 29.3 (2011), pp. 630-643. ISSN: 0733-8716. DOI: 10.1109/JSAC. 2011.110312.

[144] Bo Yu, Cheng-Zhong Xu, and Bin Xiao. "Detecting Sybil attacks in VANETs." In: Journal of Parallel and Distributed Computing 73.6 (2013), pp. 746-756. ISSN: 0743-7315. 
[145] Chenxi Zhang, Rongxing Lu, Xiaodong Lin, Pin-Han Ho, and Xuemin Shen. "An efficient identity-based batch verification scheme for vehicular sensor networks." In: Proceedings of 27 th Conference on Computer Communications. IEEE, 2008.

[146] Chenxi Zhang, Xiaodong Lin, Rongxing Lu, and P-H Ho. "RAISE: an efficient RSU-aided message authentication scheme in vehicular communication networks." In: 2008 IEEE International Conference on Communications. IEEE. 2008, pp. 1451-1457.

[147] Tong Zhou, Romit Roy Choudhury, Peng Ning, and Krishnendu Chakrabarty. "Privacy-preserving detection of sybil attacks in vehicular ad hoc networks." In: Mobile and Ubiquitous Systems: Networking \& Services, 2007. MobiQuitous 2007. Fourth Annual International Conference on. IEEE. 2007, pp. 1-8. 

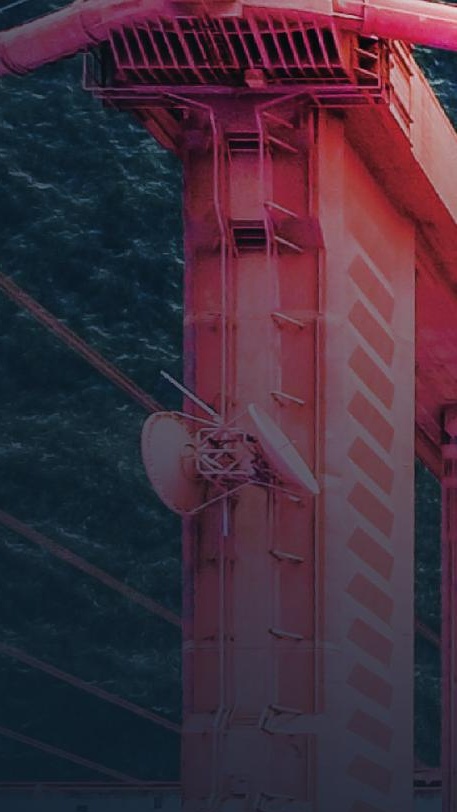